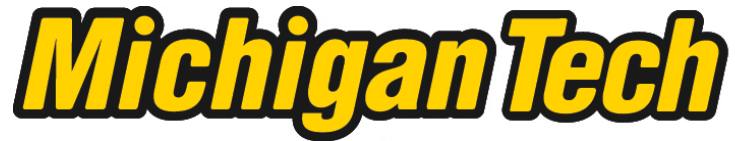 \\ Michigan Technological University Create the Future Digital Commons @ Michigan Tech
}

Dissertations, Master's Theses and Master's Reports - Open

Dissertations, Master's Theses and Master's

Reports

2013

\section{MULTISCALE MODELING OF LIQUID CRYSTALLINE/NANOTUBE COMPOSITES}

Sharil Patrale

Michigan Technological University

Follow this and additional works at: https://digitalcommons.mtu.edu/etds

Part of the Mechanical Engineering Commons, and the Nanoscience and Nanotechnology Commons Copyright 2013 Sharil Patrale

\section{Recommended Citation}

Patrale, Sharil, "MULTISCALE MODELING OF LIQUID CRYSTALLINE/NANOTUBE COMPOSITES", Master's Thesis, Michigan Technological University, 2013.

https://doi.org/10.37099/mtu.dc.etds/585

Follow this and additional works at: https://digitalcommons.mtu.edu/etds

Part of the Mechanical Engineering Commons, and the Nanoscience and Nanotechnology Commons 
MULTISCALE MODELING OF

LIQUID CRYSTALLINE/NANOTUBE COMPOSITES

By

Sharil Patrale

A THESIS

Submitted in partial fulfillment of the requirements for the degree of MASTER OF SCIENCE

In Mechanical Engineering

MICHIGAN TECHNOLOGICAL UNIVERSITY

2013

(C) 2013 Sharil Patrale 
This thesis has been approved in partial fulfillment of the requirements for the degree of MASTER OF SCIENCE in Mechanical Engineering.

Department of Mechanical Engineering - Engineering Mechanics

Thesis Advisor: Dr. Gregory Odegard

Committee Member: Dr. Ibrahim Miskioglu

Committee Member: Dr. Julia King

Department Chair: Dr. William Predebon 


\section{TABLE OF CONTENTS}

TABLE OF CONTENTS

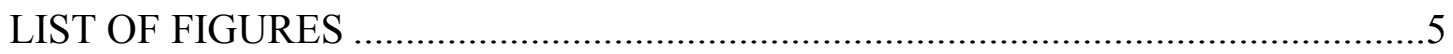

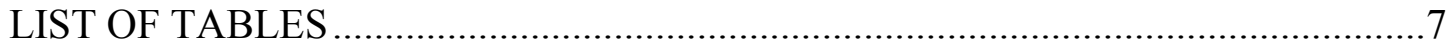

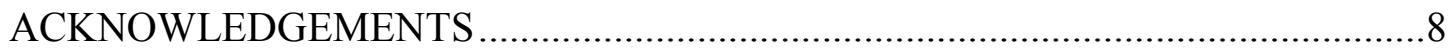

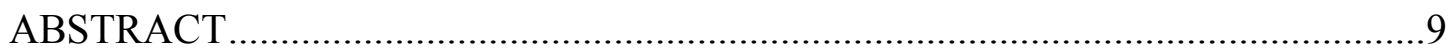

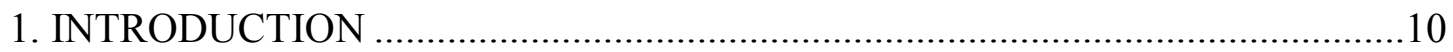

1.1 Composite Material.................................................................................12

1.2 Polymer Matrix Composites ..........................................................................12

1.3 Liquid Crystalline Polymers .............................................................................13

1.4 Carbon Nanotubes.................................................................................14

2. MICROMECHANICAL MODELING …………………….............................17

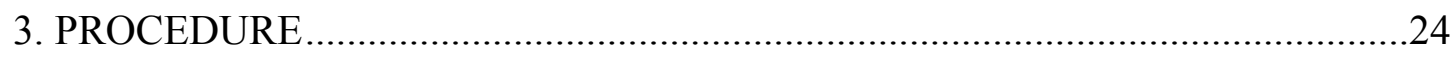

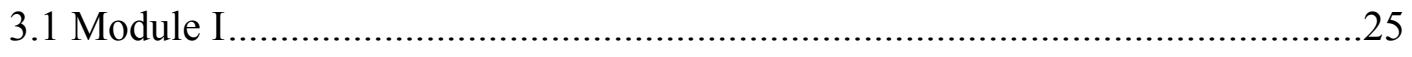

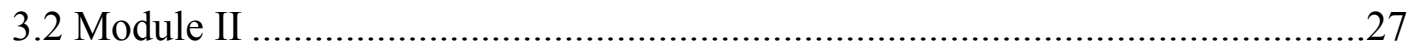

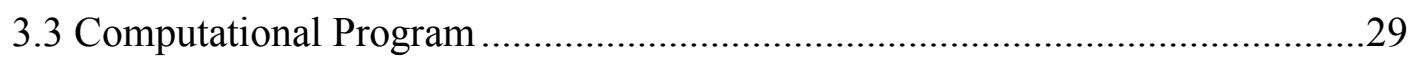

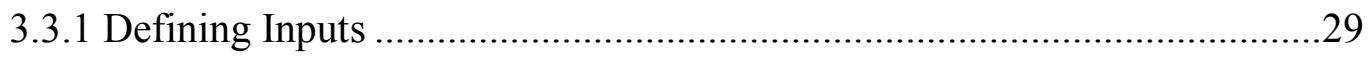

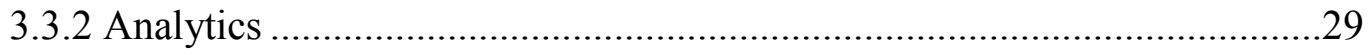

3.3.2.1 Continuum Expansions .....................................................................

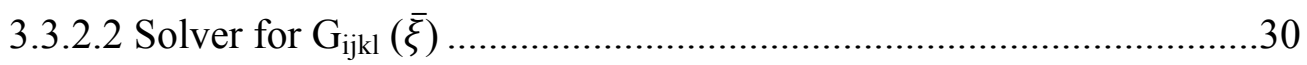

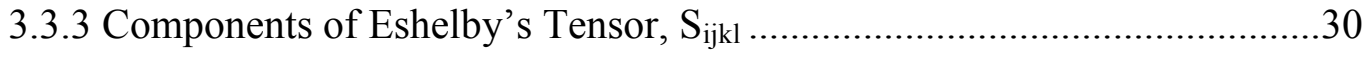


3.3.4 Mori-Tanaka Calculations

3.3.5 Combined Processing.............................................................................

3.3.6 Graphical Representation.....................................................................32

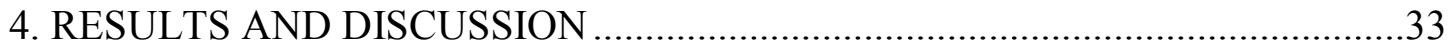

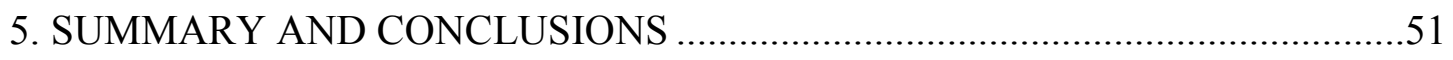

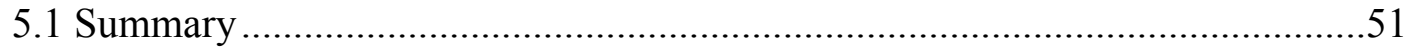

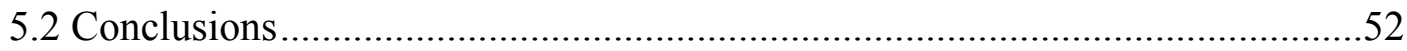

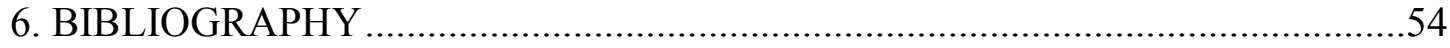

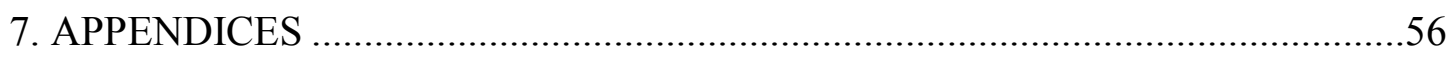

7.1 Appendix A - Material Properties …………………...................................56

7.2 Appendix B - Matlab code and Explanation .....................................................59

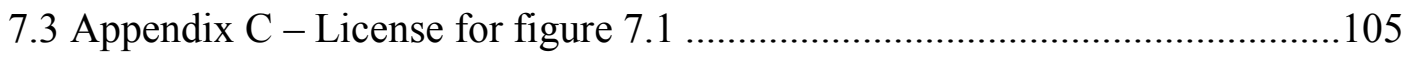




\section{LIST OF FIGURES}

1.1 Block diagram showing a cross sectional representation along the 2-3 plane of a general composite material............................

4.1 Graph showing Longitudinal Young's modulus (E1) plotted v/s the nanotube volume fraction (vf) for unidirectionally aligned fibers of various lengths

4.2 Graph showing Longitudinal Young's modulus (E1) plotted v/s the nanotube volume fraction (vf) for unidirectionally aligned fibers of various lengths at volume fractions ranging from 0 to $5 \%$

4.3 Graph showing transverse Young's modulus (E2) plotted v/s the nanotube volume fraction (vf) for unidirectionally aligned fibers of various lengths at volume fractions ranging from 0 to $5 \%$

4.4 Graph showing longitudinal shear modulus (G12) plotted v/s the nanotube volume fraction (vf) for unidirectionally aligned fibers of various lengths

4.5 Graph showing Longitudinal Young's modulus (E1) plotted against the nanotube volume fraction (vf $0-5 \%$ ) for various orientations of the the nanotubes at the length of $500 \mathrm{~nm}$....

4.6 Graph showing transverse Young's modulus (E2) plotted against the nanotube volume fraction ( $\mathrm{vf} 0-5 \%$ ) for various orientations of the the nanotubes at the length of $500 \mathrm{~nm}$

4.7 Graph showing longitudinal shear modulus (G12) plotted against the nanotube volume fraction (vf $0-5 \%$ ) for various orientations of the the nanotubes at the length of $500 \mathrm{~nm}$.

4.8 Graph showing Longitudinal Young's modulus (E1) plotted against the nanotube length $(\mathrm{nm})$ at a $5 \%$ nanotube volume fraction for various orientations of the the nanotubes.

4.9 Graph showing transverse Young's modulus (E2) plotted against the nanotube length $(\mathrm{nm})$ at a $5 \%$ nanotube volume fraction for various orientations of the the nanotubes.... 
4.10 Graph showing longitudinal shear modulus (G12) plotted against the nanotube length $(\mathrm{nm})$ at 5\% nanotube volume fraction for various orientations of the the nanotubes.

4.11 Graph showing Longitudinal Young's modulus (E1) plotted against the nanotube orientation (deg.) for various lengths of the nanotubes at $5 \%$ nanotube volume content.

4.12 Graph showing transverse Young's modulus (E2) plotted against the nanotube orientation (deg.) for various lengths of the nanotubes at $5 \%$ nanotube volume content....

4.13 Graph showing longitudinal shear modulus (G12) plotted against the nanotube orientation (deg.) for various lengths of the nanotubes at $5 \%$ nanotube volume content.

4.14 Graph showing longitudinal Young's modulus (E1) plotted against the nanotube orientation (deg.) for varying volume fractions of nanotubes of length $500 \mathrm{~nm}$.

4.15 Graph showing transverse Young's modulus (E2) plotted against the nanotube orientation (deg.) for varying volume fractions of nanotubes

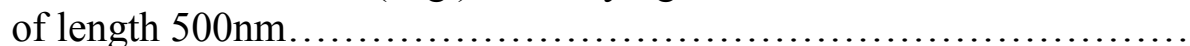

4.16 Graph showing longitudinal shear modulus (G12) plotted against the nanotube orientation (deg.) for varying volume fractions of nanotubes

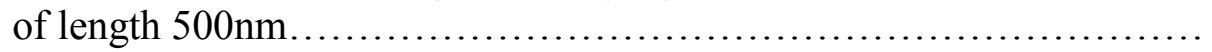

4.17 Graph showing longitudinal Young's modulus (E1) plotted against the nanotube lengths $(\mathrm{nm})$ for varying volume fractions of nanotubes aligned uniaxially with the LCP molecules........................

4.18 Graph showing transverse Young's modulus (E2) plotted against the nanotube lengths $(\mathrm{nm})$ for varying volume fractions of nanotubes aligned uniaxially with the LCP molecules

4.19 Graph showing longitudinal shear modulus (G12) plotted against the nanotube lengths $(\mathrm{nm})$ for varying volume fractions of nanotubes aligned uniaxially with the LCP molecules.

7.1 Equivalent continuum modeling of effective fiber 


\section{List of Tables}

7.1 Elastic properties of Liquid Crystalline Polymer.................... 57

7.2 Components of the stiffness tensor for effective fiber (GPa).......... 57

7.3 Stiffness matrix obtained from the model for a LCP/nanotube composite containing $25 \%$ volume concentration of $500 \mathrm{~nm}$ long nanotubes aligned uniaxially with the LC monomers................ 58 


\section{ACKNOWLEDGEMENTS}

I would like to express my deep gratitude to my advisor, Dr. Gregory Odegard, for his valuable guidance, constant encouragement and useful critiques of this research work. It was the inspiration I gained from his knowledge and experience that helped me in completing this research project successfully. I would like to thank Dr. Richard Chartoff from the University of Dayton at Oregon for supporting the research project with a NSF grant and for providing the required experimental data for the project. I am also very thankful to Dr. Ibrahim Miskioglu and Dr. Julia king for their valuable suggestions on my work and taking time to serve on my Master's thesis defense. This work would also not have been possible without the valuable inputs that I received, at the weekly seminars, from my research group. I would like to acknowledge the contribution from my friends at Michigan Tech who were always there to support and guide me with their experience and make my stay at Michigan Tech extremely joyful. I would like to take this opportunity to extend my gratitude towards my parents, Mr. Avinash Patrale and Mrs. Neha Patrale, for their immense moral and financial support during my tenure at Michigan Tech. I feel gifted to have a sister like Piyuri Patrale, who was a constant source of motivation for me to make the herculean task of thesis writing easy and tractable. 


\begin{abstract}
The objective of this research is to synthesize structural composites designed with particular areas defined with custom modulus, strength and toughness values in order to improve the overall mechanical behavior of the composite. Such composites are defined and referred to as 3D-designer composites. These composites will be formed from liquid crystalline polymers and carbon nanotubes. The fabrication process is a variation of rapid prototyping process, which is a layered, additive-manufacturing approach. Composites formed using this process can be custom designed by apt modeling methods for superior performance in advanced applications. The focus of this research is on enhancement of Young's modulus in order to make the final composite stiffer. Strength and toughness of the final composite with respect to various applications is also discussed. We have taken into consideration the mechanical properties of final composite at different fiber volume content as well as at different orientations and lengths of the fibers. The orientation of the LC monomers is supposed to be carried out using electric or magnetic fields. A computer program is modeled incorporating the Mori-Tanaka modeling scheme to generate the stiffness matrix of the final composite. The final properties are then deduced from the stiffness matrix using composite micromechanics. Eshelby's tensor, required to calculate the stiffness tensor using Mori-Tanaka method, is calculated using a numerical scheme that determines the components of the Eshelby's tensor (Gavazzi and Lagoudas 1990). The numerical integration is solved using Gaussian Quadrature scheme and is worked out using MATLAB as well. . MATLAB provides a good deal of commands and algorithms that can be used efficiently to elaborate the continuum of the formula to its extents. Graphs are plotted using different combinations of results and parameters involved in finding these results.
\end{abstract}




\section{Introduction}

The field of Nano-Composites currently stands amongst the most vibrant areas for research and accounts for various aspects of Nanotechnology viz; Polymers, Carbon Nanotubes, etc. The scope of this research project extends to synthesizing nanocomposites with the matrix phase containing Liquid Crystal Polymers (LCP) reinforced with a predetermined fraction of Carbon Nanotubes. The motivation to use carbon nanotubes for reinforcement stems from the fact that the nanotubes are approximately an order of magnitude stronger than the carbon fibers which in turn are an order of magnitude stronger than the conventional steel. The high strength to weight ratio makes the LCP/nanotube composites useful for various structural applications. The properties obtained from the final composite depend on the individual properties of the polymer, the nanotubes and the interaction phase between both.

A research program is proposed by the University of Dayton to create a new class of 3-D self assembled nano-composites with functionally graded properties. Materials of interest are formed from thermosetting, reactive liquid crystal monomers containing carbon nanotubes. A thermosetting polymer, when dissolved in a solvent or heated to its melting point exhibits liquid crystallinity and is called as Liquid Crystalline Polymer. The motivation for this research mainly lies in that the LC monomers can be oriented in preferred direction by using the electric or magnetic fields. Similar work of aligning the polymer composites of nanotubes with the help of magnetic field has been carried out by Kimura et al., (Kimura et al. 2002). The orientation is then locked in by photocuring the monomers in-situ. It is believed that the LC monomers offer various advantages that have not been explored yet with respect to nano-composites: ability to align the LC monomer in preferred axial directions and simultaneously crosslinking the material to lock the alignment, the capability for self assemblage attributed to the large surface to volume ratios involved are some of these advantages. 
Consequently, the anisotropy introduced by the varying 1-D alignment of the nanotubes makes it possible to make nano-composites with three dimensional variations in properties. Such localized alignment cannot be achieved with non LC monomers. Another advantage is that the low viscosity of the LC polymer aids the dispersion of carbon nanotubes. Again, the LC monomers cure rapidly under single light exposures. Other advantages of LCP over the non-LCP are low viscosity, high glass transition temperature, 120-200 degrees C, and thermal/oxidative stability because of its rigid rod character. The nano-composites are formed via an inkjet deposition (IJD) process so that local variations in alignment as well as composition can be accommodated. They are photocured by exposure with ultraviolet radiation delivered by a focused, compact LED light source. Orienting LC monomers containing nanotubes causes self-alignment of the nanotubes, resulting in composites with enhanced anisotropic mechanical properties.

The composites will be fabricated using a variation of the rapid prototyping process, which is a layered, additive-manufacturing approach. The RP process involves a 3D CAD modeling of an object that initiates the automated production of its physical form by a continuous layer-by-layer deposition of material. Such deposition technique will allow the direct production of high-performance composites without the use of conventional manufacturing tools like forging, machining or molding. This technology offers great potential for applications at remote locations and on ships at sea for cultivation of one-of-a-kind critical components. It also offers great potential for future applications in space programs for extraterrestrial production of spare parts. If this type of process were adapted to space it would offer a great advantage for fabrication of unique composite components if and when they are required on the space vehicle. 


\subsection{Composite Materials}

A material, manufactured or occurring naturally, having two or more distinct constituent materials that exhibit unique properties is called a composite material. Consequentially, properties obtained from the final material are a combination of the properties of its constituent materials. The composite materials, thus exhibit enhanced properties such as high stiffness, high strength etc. and are useful in a wide scope of applications ranging from nanotechnology to space technology. The constituent materials in a composite can be characterized into two main categories which are the matrix and the fiber or the reinforcement. Depending on the type of matrix material used, the composites are categorized in three main types: metal matrix composites, polymer matrix composites and ceramic matrix composites.

\subsection{Polymer Matrix Composites}

A composite material with polymer as the matrix phase is known as polymer matrix composite (PMC). The polymer matrix composites show important significance as structural components in many applications. Their specific strength and modulus surpass the best of materials. Various applications incorporate the use of anisotropic fiber filled composites that have very high strength and stiffness in one direction. On combining with carbon fibers the polymer/fiber composites show exceptional mechanical properties which are of great importance in aerospace field. The PMCs are classified into two subgroups: the thermoplastics and the thermosets. The subgroup of interest for this research is the thermosetting polymers. Thermosetting polymers are synthetic materials that can be strengthened or hardened by heating, but cannot be remolded or reheated after their initial heat-forming and thus, they retain their shape and strength even when heated. This property helps in applications where permanent solid components need to be produced. 


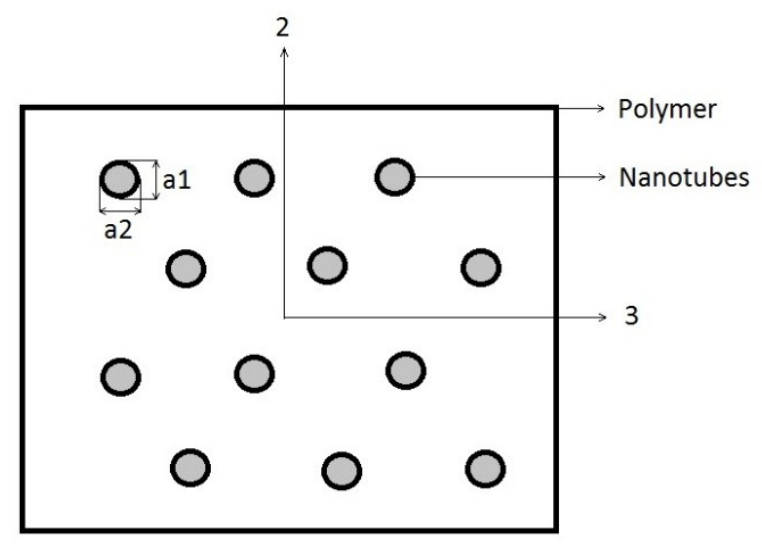

Figure 1.1: Block diagram showing a cross sectional view along the 2-3 plane of a general composite material

The directions 2 and 3 in the sketch above represent the transverse directions of loading of the composite material. The axial direction of loading or the direction 1 is perpendicular to the plane 2-3, i.e., perpendicular to the plane of the paper and along the length of the nanotubes for a general composite material.

\subsection{Liquid Crystalline Polymers}

As stated earlier, thermosetting polymers, when dissolved in a solvent or heated to their melting point exhibit liquid crystallinity and are called as liquid crystalline polymers (LCP). Exceptional physical properties and thermal stability are some of the attributes of the LC resins. Uniaxial alignment or preferred mechanical and chemical properties can be obtained from LC resins by subjecting them to magnetic fields of medium or high strengths, and this property of the LCPs to be aligned in the required direction has given rise to curiosity in using LCPs for developing structural nanocomposites. Once the required orientation is achieved, the direction of 
orientation of LCPs remains intact even after cooling them below the melting temperature (Wang et al. 2003).

The objective of this research is to create fully functional LC nano-composites with graded structures by inkjet printing. The proposed effort is build on the results derived from the NER project. The materials selected will be similar to Monomer 1 which is an acrylate terminated mesogen which has a nematic LC phase above its crystalline melting point. Photocuring of Monomer 1 is carried out successfully in both nematic as well as isotropic phases. LC alignment was accomplished with a magnetic field of modest strength (0.38 Tesla) imposed on the resin during cure. It was found that by proper choice of the molecular alignment in the LC polymer layers, physical and mechanical properties can be optimized. Methods for aligning nanotubes are of great importance because obtaining optimum properties from the reinforcing fibers requires control of fiber alignment. It is achieved by orienting the LC monomer with the help of electric or magnetic fields. The surface tension between the LC monomer and the CNTs align the CNTs in the required direction. Schultz and Chartoff studied the dynamics of the relaxation process in the nematic phase showing that the orientation imposed by a continuous magnetic or electric field persists and does not relax until the field is removed. This allows enough time for crosslinking to lock in the nanotube alignment. Using such fields and changing the orientation allows for custom alignment where different orientations within a layer can be achieved. Such alignment cannot be done with a non-LC monomer. Evidence that LC alignment works was provided by the results of "Lynch and Patrick" which made use of a nematic phase LC monomer under the influence of a field to orient suspended CNTs.

\subsection{Carbon Nanotubes}

While studying the synthesis of fullerenes in the year 1991, carbon nanotubes were discovered by Lijima, and because of their exceptional physical and thermal 
properties, that no other material has ever exhibited, they gained significant importance in the field of science and engineering. In other words, these properties make nanotubes ideal for a wide range of applications not only in the field of engineering, but also in medical applications and treatment of cancer (R.H. Baughman et al. 2002). Due to the advantages of using carbon nanotubes in developing structural materials, they are considered ideal to be used as fillers in advanced composites. When graphene sheets are rolled at proper angles, nanotubes are formed. The angle of rolling and diameter of the cylinder define the properties of the nanotube. Further, depending on the number of graphene sheets used, nanotubes may be classified as single walled nanotubes (SWNT- nanotubes having one graphene sheet) and multi walled nanotubes (MWNT- nanotubes with multiple graphene sheets rolled together). CNTs have the highest tensile strength and elastic modulus and thus, are the strongest and the stiffest materials discovered to date. The tensile strength of CNTs is found to be approximately $63 \mathrm{Gpa}$ (Min-Feng Yu et al. 2000) which is supposed to be the result of $\mathrm{sp}^{2}$ covalent bond existing between individual carbon atoms. The great interest in using nanotubes as reinforcing agents is fueled by the ability of the nanotubes to exhibit elastic modulus in excess of $1 \mathrm{TPa}$ combined with high aspect ratios of over 1000. It is expected that with such exceptional properties substantial final effects may be achieved with very low nanotube volume fractions. Thus polymer nanotube composites with tremendous strength and high stiffness, but lighter in weight than the carbon fiber-epoxy composites could be formed. Nanotubes are 100 times stiffer than the standard carbon fibers and thus, even $1 \%$ by volume of carbon nanotubes in a thermoset matrix can produce exceptional results. They are expected to produce modulus values equivalent to the $35 \%$ (vol) of standard carbon fibers in a typical epoxy composite.

This research employs multi-walled carbon nanotubes because of their impressive properties. Their elastic modulus is in excess of $1 \mathrm{TPa}$. For the composites considered in this study, the fiber properties used are the elastic stiffness components, volume 
fraction, length and orientation of the fibers in the composite. The LC monomers and nanotubes are initially aligned in the 1 direction and then the nanotubes are oriented slowly from 0 degree orientation to 90 degree orientation to calculate the properties of the final composite in various cases. In order to develop simplicity in generating the Eshelby's tensor for the material, the effective fibers were assumed to be spheroidal. This part of the research emphasizes on modeling a method to calculate the properties of a composite using MATLAB. Thus, the composite studied using the model developed in this research contains material similar to Monomer 1 in the matrix form and spheroidal carbon nanotubes in the fiber form. The properties of these constituent elements along with the Eshelby's tensor are used to compute the final properties of the LCP/nanotube composites. The elastic constants for the final composite are derived using the MATLAB model for various nanotube volume fractions, lengths and orientations. Thus, the data generated using the model developed here gives an overall idea about the behavior of the final composite and is presented in the results section. 


\section{Micromechanical Modeling}

The mechanical analysis of a composite material to its constituent level is necessary in order to determine its attributes that could be of importance in various applications. This study of materials is known as micromechanics. Such properties are often too tedious to calculate using experimental methods. Clearly, inhomogeneous materials such as composites that are formed from different constituent elements exhibit properties that are a combination of the properties of its individual constituents. The task of determining the properties of the composite material, thus, takes into account the physical characteristics and properties of the individual phases and is called homogenization. Continuum micromechanics has the ability to determine the properties and behavior of inhomogeneous materials along various axial directions.

The Mori-Tanaka method entails the use of Eshelby's tensor to account for cylindrical inclusions. Here, we assume the fiber-interphase problem using a composite with cylindrical fiber inclusions. The Mori Tanaka method is a popular standard tool and is useful in determining the accuracy of the approximate solution. The main advantage with Mori-Tanaka method is that it directly computes the elastic properties of a composite in terms of its stiffness tensor or compliance tensor, which can then be used to determine effective properties by simple arithmetic calculations. Earlier, applications of this theory were linked with eigen strain, equivalent inclusions and back stress concepts (Gavazzi and Lagoudas 1990). However, this research is based on applying direct approach to define and compute the elastic moduli of composites. This research synthesizes composites with transversely isotropic elastic phases consisting of an inclusion phase of aligned or oriented ellipsoidal nanotubes. The concept of equivalent inclusion developed by Mori and Tanaka (Mori and Tanaka 1973) is used to compute the effective properties of the nano-composites. This assumes that the composite is being subjected to homogeneous boundary conditions. 
The averaging techniques used to compute the effective properties of composite materials are based on the solution to the calculation of Eshelby's tensor for the type of inclusion for each phase of the composite. The solution to the problem of ellipsoidal inclusions has been formulated by Eshelby (J.D. Eshelby 1957) and the determination of effective elastic constants using Eshelby's formulation depends on a fourth order tensor that relates the average strain in the fibers to the average strain in the matrix, known as the Eshelby's tensor (Eshelby 1957). This Eshelby's tensor is given as $\mathrm{S}_{\mathrm{ijkl}}$. The way in which these tensors are approximated is elucidated in the framework provided by Benveniste (Y. Benveniste 1987). The Eshelby's tensor provides a direct formulation of the method to find effective moduli: a method which eliminates the use of eigen strain, back stress and energy concepts and develops a closed loop form expression. The way in which the Eshelby tensor relates the average strain in the fibers to the average strain in the matrix is given by Eshelby (J.D. Eshelby 1957).

The modeling part of this research project consists of application of the Mori-Tanaka averaging method in order to predict the effective moduli of nano-composites with liquid crystal polymer in the matrix phase and the inclusions phase consisting of carbon nanotubes that are assumed to be of the same size and shape. The analysis is carried out at different lengths and orientations of CNTs. The micromechanics method used in this research to predict the effective moduli is based on Mori-Tanaka averaging method (Mori and Tanaka 1973) and has previously been used to compute properties of composites with transversely-isotropic inclusions by Qui and Weng (Qui and Weng 1990). This technology has been used in modeling to design composites that would then be synthesized by the IJD process. The multiscale design procedure developed by Odegard has been used and combines quantum molecular dynamics and coarse-graining modeling tools to simulate the material structure and behavior at different length scales of the nanotubes. This modeling method incorporates micromechanical analyses for the prediction of the behavior occurring from the bulk 
mechanical properties of nanotube/polymer composites with various compositions of the nanotubes in the composite.

Combination of micromechanics and continuum mechanics is essential in order to synthesize a multiconstituent material. Continuum mechanics assumes homogeneity in material properties. Continuum mechanics' assumption of uniform material properties might not hold at the micro level and thus, the continuum quantities associated with the micro level constituents of the material, are expressed with the help of micromechanics (G. M. Odegard, et al. 2005). In this study, effective fiber was taken into consideration instead of considering the nanotube/polymer interface separately. The effective fiber was supposed to comprise the molecules surrounding the interface of the constituent materials. Consequently, the computation of the properties for effective fibers indirectly explains the behavior of the interface molecules under different situations (G.M. Odegard, et al. 2005). Thus, perfect bonding can be assumed to exist between LC/nanotube effective fibers and the surrounding LC matrix in the micromechanics analysis (G. M. Odegard, et al. 2005).

The elastic mechanical properties of the composite material were predicted with the help of a micromechanics based model; the Mori-Tanaka method (Mori T, Tanaka K 1973). The elastic stiffness matrix for the composite material, using this method, is given by Benveniste (Y. Benveniste 1987).

$C=C^{m}+v_{f}\left\{\left(C^{f}-C^{m}\right) A^{f}\right\}\left(v_{m} I+v f\left\{A^{f}\right\}\right)^{-1}$

Where $\mathrm{C}^{\mathrm{m}}$ is the matrix stiffness tensor, $\mathrm{C}^{\mathrm{f}}$ is the fiber stiffness tensor, $\mathrm{v}_{\mathrm{f}}$ is the fiber volume fraction, $\mathrm{v}_{\mathrm{m}}$ is the matrix volume fraction, $\mathrm{I}$ is the fourth order identity tensor and the orientation dependant tensor $\mathrm{A}^{\mathrm{f}}$ is the dilute strain concentration factor for the fiber given in eq. (2.2). The stiffness matrix, $\mathrm{C}$, obtained from using nanotubes of 
length $500 \mathrm{~nm}$ and for a volume content of $25 \%$ and aligned in the same direction as that of the polymer is given in the Appendix section A of this document.

$A^{f}=\left[I+S\left(C^{m}\right)^{-1}\left(C^{f}-C^{m}\right)\right]^{-1}$

S denotes the fourth order Eshelby's tensor and is given by Eshelby (J. D. Eshelby 1957) and Mura (T. Mura 1982). For a general anisotropic material with an ellipsoidal inclusion embedded in its matrix, the Eshelby tensor is given in terms of a double integration (Gavazzi and Lagoudas 1990) that has to be carried out numerically as depicted in this document. The analytical approach to such a problem in finite element program will require a double numerical integration at varied points in the material for every load increment (Gavazzi and Lagoudas 1990). Here we used the Gaussian Quadrature program developed by Gavazzi and Lagoudas with variable number of integration points to evaluate the Eshelby's tensor. A computer program is developed with the help of MATLAB to solve the double integration problem. The results obtained from the program are then fed into the Mori-Tanaka averaging scheme discussed before to evaluate the effective mechanical properties of $\mathrm{LCP} /$ nanotube composites.

If an ellipsoidal inclusion is subjected to a uniform, stress-free transformation strain $\varepsilon_{\mathrm{ij}}{ }^{\mathrm{T}}$ the resulting strain field is uniform and is represented by the Eshelby tensor $\mathrm{S}$ (J. D. Eshelby 1957) as:

$\varepsilon_{\mathrm{ij}}^{\mathrm{c}}=\mathrm{S}_{\mathrm{ijkl}} \varepsilon_{\mathrm{kl}}^{\mathrm{T}}$

where, $\mathrm{S}_{\mathrm{ijkl}}$ represents the components of $\mathrm{S}$ which can be predicted with the help of the stiffness tensor, L, of the matrix and the shape of the fiber. 
The Eshelby's tensor for the case of a general anisotropic tensor is given by the following double integration which depicts the surface of the unit sphere (Mura 1987).

$\mathrm{S}_{\mathrm{ijkl}}=\frac{1}{8 \pi} \mathrm{L}_{0 \mathrm{mnkl}} \int_{-1}^{+1} d \xi_{3} \int_{0}^{2 \pi}\left\{\mathrm{G}_{\mathrm{imjn}}(\bar{\xi})+\mathrm{G}_{\mathrm{jmin}}(\bar{\xi})\right\} \mathrm{d} \omega$

Where

$\mathrm{G}_{\mathrm{ijkl}}(\bar{\xi})=\xi_{\mathrm{k}} \xi_{\mathrm{l}} \mathrm{N}_{\mathrm{ij}}(\bar{\xi}) / \mathrm{D}(\bar{\xi})$

$\bar{\zeta}_{l}=\zeta_{\mathrm{i}} / \mathrm{a}_{\mathrm{i}} ; \zeta_{1}=\left(1-\zeta_{3}{ }^{2}\right)^{1 / 2} \cos \omega ; \zeta_{2}=\left(1-\zeta_{3}{ }^{2}\right)^{1 / 2} \sin \omega ; \zeta_{3}=\zeta_{3}$

$\mathrm{D}(\bar{\xi})=\varepsilon_{\mathrm{mnl}} \mathrm{K}_{\mathrm{m} 1} \mathrm{~K}_{\mathrm{n} 2} \mathrm{~K}_{13}$

$\mathrm{K}_{\mathrm{ik}}=\mathrm{L}_{0 \mathrm{ijkl}} \xi_{\mathrm{j}} \xi_{1}$

$\mathrm{N}_{\mathrm{ij}}(\bar{\xi})=\frac{1}{2} \varepsilon_{\mathrm{ikl}} \varepsilon_{\mathrm{jmn}} \mathrm{K}_{\mathrm{km}} \mathrm{K}_{\mathrm{ln}}$

The set of eq. (2.5) follow continuum expansions. These equations can be elaborated so as to solve for obtaining the components of the Eshelby's tensor from eq. (2.6). We can write eq. (2.5.4) as,

$\mathrm{K}_{11}=\mathrm{L}_{1111} * \xi_{1} * \xi_{1}+\mathrm{L}_{1212} * \xi_{2} * \xi_{2}+\mathrm{L}_{1313} * \xi_{3} * \xi_{3}+\mathrm{L}_{1112} * \xi_{1} * \xi_{2}+\mathrm{L}_{1113} *$

$\xi_{1} * \xi_{3}+\mathrm{L}_{1211} * \xi_{2} * \xi_{1}+\mathrm{L}_{1213} * \xi_{2} * \xi_{3}+\mathrm{L}_{1312} * \xi_{3} * \xi_{2}+\mathrm{L}_{1311} * \xi_{3} * \xi_{1}$

and eq. (2.5.5) as,

$$
\begin{aligned}
& \mathrm{N}_{11}(\bar{\xi})=\left(\varepsilon_{123} * \varepsilon_{231} * \mathrm{~K}_{23} * \mathrm{~K}_{31}+\varepsilon_{132} * \varepsilon_{213} * \mathrm{~K}_{31} * \mathrm{~K}_{23}+\varepsilon_{123} * \varepsilon_{213} * \mathrm{~K}_{21} * \mathrm{~K}_{33}\right. \\
& \left.+\varepsilon_{132} * \varepsilon_{231} * \mathrm{~K}_{33} * \mathrm{~K}_{21}\right) / 2
\end{aligned}
$$

Also eq. (2.5.3) as,

$\mathrm{D}(\bar{\xi})=\varepsilon_{123} * \mathrm{~K}_{11} * \mathrm{~K}_{22} * \mathrm{~K}_{33}+\varepsilon_{231} * \mathrm{~K}_{21} * \mathrm{~K}_{32} * \mathrm{~K}_{13}+\varepsilon_{312} * \mathrm{~K}_{31} * \mathrm{~K}_{12} * \mathrm{~K}_{23}+$

$\varepsilon_{132}{ }^{*} \mathrm{~K}_{11} * \mathrm{~K}_{32} * \mathrm{~K}_{23}+\varepsilon_{213} * \mathrm{~K}_{21} * \mathrm{~K}_{12} * \mathrm{~K}_{33}+\varepsilon_{321} * \mathrm{~K}_{31} * \mathrm{~K}_{22} * \mathrm{~K}_{13}$ 
Using these equations along with the other equations in eq. (2.5.2), the values of $\mathrm{G}_{\mathrm{ijkl}}(\bar{\xi})$ can be calculated for various combinations of $\mathrm{i}, \mathrm{j}, \mathrm{k}$ and $\mathrm{l}(\mathrm{i}=\mathrm{j}=\mathrm{k}=\mathrm{l}=1$, $2,3)$ as follows:

$\mathrm{G}_{1111}=\bar{\xi}_{1} * \bar{\xi}_{1} * \mathrm{~N}_{11}(\bar{\xi}) / \mathrm{D}(\bar{\xi})$

Similarly, eq. (2.5.1) can be solved for all 81 components of $G(\bar{\xi})$. Once these components are obtained, eq. (2.6) can be solved for all the components of $S_{\mathrm{ijkl}}$. Refer Appendix B for the expansion of the above equations over all the continuum points. The expansion of eq. (2.6) is also presented in the same section of the appendix.

In the above equations, $\varepsilon_{\mathrm{ijk}}$ is the permutation tensor and $\mathrm{L}_{0 \mathrm{ijk}}$ the components of stiffness tensor $\mathrm{L}_{0}$. The solution for eq. (2.4) has been given in Mura (1987) for general cases such as isotropic and transversely isotropic materials. No explicit formula for anisotropic materials exists that gives the components of the Tensor S. According to the author, the program's accuracy for the computation depends upon the aspect ratios $a_{1} / a_{2}$ and $a_{1} / a_{3}$ (Gavazzi and Lagoudas 1990). The double integration in eq. (2.4) can be solved by numerical integration using the Gaussian quadrature formula (Gavazzi and Lagoudas 1990)

$\mathrm{S}_{\mathrm{ijkl}}=\frac{1}{8 \pi} \sum_{p=1}^{M} \sum_{q=1}^{N} L_{0 m n k l}\left\{\mathrm{G}_{\mathrm{imjn}}\left(\omega_{\mathrm{q}}, \zeta_{3 \mathrm{p}}\right)+\mathrm{G}_{\mathrm{jmin}}\left(\omega_{\mathrm{q}}, \zeta_{3 \mathrm{p}}\right)\right\} \mathrm{W}_{\mathrm{pq}}$

where, $\mathrm{M}$ and $\mathrm{N}$ are the integration points for the Gaussian quadrature scheme in integrating over $\zeta_{3}$ and $\omega$, respectively, and vary with the aspect ratios $a_{1} / a_{2}$ and $a_{1} / a_{3}$. The term $\mathrm{W}_{\mathrm{pq}}$ represents the Gaussian weights that are taken from a handbook of Mathematical functions. Since, cylindrical inclusions are being considered, the ratio $a_{1} / a_{2}$ is set to one and remains unchanged, whereas, the ratio $a_{1} / a_{3}$ varies as per the length of the inclusions where $a_{3}$ represents the length of an inclusion. 
The modeling part of this research deals with developing a computer program to accurately and efficiently solve the eq. (2.6) in order to compute the components of Eshelby's tensor, S, which has been previously defined. As has been said before, Ghahremani's computer program is the only solution that exists as far as $\mathrm{S}$ for anisotropic composites is concerned. An effort is made to develop an efficient solution that would generate the Eshelby's tensor for a generic anisotropic material. The results from this solution would then be fed to the Mori-Tanaka scheme that has been briefed previously and the entire model would ultimately be used to design composites. These composites would then be synthesized by the IJD process. 


\section{Procedure}

The aim of this research was to develop a mathematical model that would efficiently predict the effective properties of the $\mathrm{LCP} /$ nanotube composites. The development of this mathematical model was divided into two different modules. The first module consisted of developing a model for the general Mori-Tanaka equations that could predict the effective mechanical properties of isotropic or transversely isotropic polymer/nanotube materials. It was noted that this model was insufficient when the material in play showed anisotropic properties. Since their properties vary at every point in the material, it is difficult to compute the Eshelby's tensor for anisotropic materials in the same fashion that is used for the transversely isotropic materials. A numerical scheme developed by Gavazzi and Lagoudas (Gavazzi and Lagoudas 1990) was selected to compute the Eshelby's tensor for such materials. Since the disintegration of this scheme to find components of the Eshelby's tensor for anisotropic materials required continuum mechanics based expansion of surface integral equations, a separate mathematical model, dedicated entirely to the development of Eshelby's tensor, was created. This constituted the second module in the development of the mathematical model. The last step in modeling the process to determine the effective properties of the composite consisted of the merger of the two mathematical models developed using MATLAB. Thus, the resulting model consisted of the bulk matrix, effective fiber properties, fiber dimensions, fiber volume fraction and orientation as the variables. Using these variables, the model was capable of computing the Eshelby's tensor S employing the eq. (2.5) and (2.6), which was ultimately used to generate the stiffness matrix for the LC/nanotube composite using the eq. (2.1) and (2.2). Considering the complexity of the idea and the broad range of continuum based expansion involved, the synthesis of the continuum-based expansions and the final model are explicitly explained in the following section. 
Accounting for the relationships between the structure of the composite and its properties at the nanoscale interphase between the polymer and fibers was very important and thus, the concept of effective fiber was defined by Odegard (G. M. Odegard, et al. 2003). The effective fiber, which can be directly modeled using continuum mechanics, perfectly defines these relationships at such minute scales. This study consisted of the use of mechanical properties of the effective fiber and the bulk polymer matrix for the polymer/nanotube composite. The LC molecules surrounding the interphase region were included in the effective fiber whereas, the rest of the polymer molecules constituted to the bulk mechanical properties of the matrix. Even though the properties varied, perfect bonding was assumed to exist between the effective fiber and the polymer matrix. It was possible, therefore, to develop a constitutive model of the composite for micromechanical analysis. This study was carried out at varying fiber volume fractions and lengths of fiber. The length of the nanotube and the effective-fiber remained equal whereas, the nanotube volume fraction was calculated to be $34 \%$ of the effective-fiber volume fraction (G.M. Odegard, et al. 2003).

\subsection{Module I}

Due to the complex nature of the work involved, the modeling procedure was divided into the aforementioned modules. This ensured accuracy in generating final results. The initial step in this process was to validate the results from the model developed. Since, the Mori-Tanaka method developed by Qui and Weng (Qui and Weng 1990) for isotropic and transversely isotropic materials has been extensively used by Odegard in the modeling of nanotube-reinforced polymer composites (G.M. Odegard, et. al 2003), it was decided that an attempt would be made to replicate his results. A MATLAB model was developed to incorporate the eq. (2.1) and (2.2) given by Weng (Qui and Weng 1990). The Eshelby's tensor, S, depends upon the type of the material as well as on the shape and size of the inclusions. The terms $\mathrm{C}^{\mathrm{f}}$ and $\mathrm{C}^{\mathrm{m}}$ are matrices of 
order 6 , as has been mentioned in the preceding section, and denote the mechanical properties of the fiber and the matrix which are taken directly from Odegard's paper (G.M. Odegard, et. al 2003). These properties are given in table 7.1 and 7.2 of the appendix. Since, here, isotropic/transversely isotropic materials are under consideration, the matrix of order 9 can be replaced by a matrix of order 6 because of symmetry in the stress and strain tensors. This results in simplicity in solving equations and prevents singularity from arising in the stiffness matrix $\mathrm{C}$ for the final composite in eq. (2.1).

While developing a Matlab model for Mori-Tanaka method to synthesize transversely isotropic materials, the first step was to define all the parameters that were to be used in the development of the program. These included specifying the stiffness matrices of matrix material and effective-fibers, the length and diameter of the inclusions or the aspect ratio, the Eshelby's tensor for transversely isotropic materials which was developed using a set of equations developed by Qiu and Weng (Qui and Weng 1990). Once all the parameters were defined, the eqs (2.1) and (2.2) were modeled to be solved for different volume fractions of the nanotubes. This was achieved by establishing a "for" loop that defined the range of the fiber volume fractions at which the mechanical properties of the polymer/nanotube composite were to be determined. The entire procedure is explained in detail in Appendix B. It is of significance to note here that the stiffness matrix for the effective fiber was directly available from the work of Odegard (G.M. Odegard, et al. 2003) whereas, the stiffness matrix for the polymer matrix used in Odegard's work was calculated from a set of equations from the mechanics of composite materials. Thus, a compliance matrix was first obtained using the equations given in appendix and the inverse of this matrix gave the stiffness matrix for the polymer. Since, the Eshelby's tensor is sensitive to aspect ratio of the nanotubes, a separate code was written to incorporate the changes in length of the nanotubes. Once the code was executed, the command window displayed required results for the range of volume fraction used in the "for" loop. These results included 
and were not limited to mainly the elastic constants of the composite material. Graphs of longitudinal Young's modulus, $E_{1}$ against the corresponding fiber volume fraction were plotted for varying lengths of the nanotubes.

Upon comparison it was found that the results obtained from the Mori-Tanaka equations formulated using MATLAB were in accordance with those obtained by Odegard et al., (G.M. Odegard, et al. 2003) for single walled nanotube-reinforced polyimide composite systems. This work established the validity of the code developed to solve the Mori-Tanaka equations for the stiffness matrix. The validity of the MATLAB code was tested for various variables such as fiber orientations aligned, random and at different angles, fiber volume fraction ranging between $10 \%$ and $90 \%$ by volume, and fiber lengths ranging between $10 \mathrm{~nm}$ and $500 \mathrm{~nm}$.

\subsection{Module II}

As stated earlier, module II consists of developing a Matlab program to calculate the Eshelby's tensor for a general anisotropic material. A numerical scheme developed by Gavazzi and Lagoudas (Gavazzi and Lagoudas 1990) was coded with the help of MATLAB and the program was executed to generate Eshelby's tensor $\mathrm{S}_{\mathrm{ijk} \text { l. }}$ Since the properties vary at every point in an anisotropic material, the method given by Qiu and Weng (Qui and Weng 1990) to calculate components of $S$ is not suitable. The components of $\mathrm{S}_{\mathrm{ijkl}}$ in the method given by Gavazzi and Lagoudas (Gavazzi and Lagoudas 1990) are incorporated by the eq. (2.6) given previously, and are defined by the continuum expansion of the double integration over the surface of the effective fiber. $\mathrm{S}_{\mathrm{ijkl}}$ represents 81 components of a matrix of order 9 which are required to define the stress strain interaction at the interphase completely.

The task of the Module II is to take input from the variable $\mathrm{L}_{0}$ which is the stiffness tensor for the matrix material, the length and the diameter of the nanotubes and use 
these data points to solve a set of equations defined in eq. (2.5). After meticulously solving each eq. from (2.5), the values for the components of $\mathrm{G}_{\mathrm{ijkl}}(\bar{\xi})$ are obtained which form a part of the eq. (2.6). Once these components are determined, the double summation in eq. (2.6) is solved over predefined number of Gaussian integration points. The number of these integration points is based on the aspect ratio $a_{1} / a_{2}$ of the inclusions. Once these values were obtained, eq. (2.6) was expanded with the help of continuum mechanics. Eq. (2.6) constitutes for three types of summations defined as follows: continuum expansion of the terms $\mathrm{G}_{\mathrm{imjn}}\left(\omega_{\mathrm{q}}, \zeta_{3 \mathrm{p}}\right)$ and $\mathrm{G}_{\mathrm{jmin}}\left(\omega_{\mathrm{q}}, \zeta_{3 \mathrm{p}}\right)$, summation of the equation $\mathrm{L}_{0 \mathrm{mnkl}}\left\{\mathrm{G}_{\mathrm{imjn}}\left(\omega_{\mathrm{q}}, \zeta_{3 \mathrm{p}}\right)+\mathrm{G}_{\mathrm{jmin}}\left(\omega_{\mathrm{q}}, \zeta_{3 \mathrm{p}}\right)\right\} \mathrm{W}_{\mathrm{pq}}$ over "q" ranging from 1 to $\mathrm{N}$ and finally summation of the equation $\sum_{q=1}^{N} L_{0 \mathrm{mnk}}\left\{\mathrm{G}_{\mathrm{imjn}}\left(\omega_{\mathrm{q}}, \zeta_{3 \mathrm{p}}\right)\right.$ $\left.+\mathrm{G}_{\mathrm{jmin}}\left(\omega_{\mathrm{q}}, \zeta_{3 \mathrm{p}}\right)\right\} \mathrm{W}_{\mathrm{pq}}$ over "p" ranging from 1 to $\mathrm{M}$. The most challenging part of the computational problem was to develop a program that would account for all the continuum expansions which would then be used in these summation equations to generate the Eshelby's tensor for a generic anisotropic material.

Ultimately, as the program to generate S was established, the code in Module II was merged with the code in Module I thus giving an efficient program that would calculate the elastic constants for a general composite material depending upon the fiber volume fraction, the fiber length and shape and the fiber orientation. The final program consists of three important divisions: the inputs which are constants defined by the type of the material used, computing Eshelby's tensor and using the inputs and Eshelby's tensor in the Mori Tanaka method to generate the elastic properties of the required composite material. These steps are defined in detail in the following section. 


\subsection{Computational Program}

This section is dedicated to the explanation of the important steps involved in processing the MATLAB code to define the properties of a general composite material. These steps are as follows.

\subsubsection{Defining inputs}

The inputs used for running the code are the mechanical properties of the matrix material and the inclusions, provided to the program in the form of stiffness matrices of order 9 and the aspect ratios of the inclusion material defining its size and shape. This also includes defining any other constants that would be encountered later in the program such as the values for the permutation tensors. Also, a matrix containing all the values of the Gaussian weights is defined to be used in the double summation equation. These inputs form the foundation of calculating the required attributes and are fed to the solver for the values of $G$ that would be used in the eq. (2.6).

\subsubsection{Analytics}

Some of the inputs defined earlier such as the stiffness tensor for the matrix material and the aspect ratio for the inclusions are separated out from other inputs and are used to pre-calculate some of the terms that would be used in solving the eq. (2.6) for generating the components of $\mathrm{S}$. This eases the process of solving the complex equation and makes the code more efficient. Conversely, if nested loops that compute everything at the same time are used, the computation time is comparatively more and the efficiency of the code reduces. 


\subsubsection{Continuum expansions}

The solver that computes values of $\mathrm{G}$ is based on a set of equations defined by eq. (2.5) and denoted by indicial notations which are based on continuum expansions. Again, in order to make the program more efficient, these expansions are defined beforehand so that $\mathrm{G}$ values can be computed with ease. These include the expansion of the equations for $\mathrm{K}_{\mathrm{ik}}, \mathrm{N}_{\mathrm{ij}}(\bar{\xi})$ and $\mathrm{D}(\bar{\xi})$ and are explicitly defined in the appendix section B.

\subsubsection{Solver for $G_{i j k l}(\bar{\xi})$}

Once the inputs for the program are defined, a double "for" loop is defined that computed the values for the various components of $G$ in $G_{i j k l}(\bar{\xi})$. The loop ranges from the values of $i=1$ to $N$. The loop is divided into two parts: one that takes the positive value of eta3 $\left(\zeta_{3}\right)$ into account and the other that takes the negative value into account. This is done with the help of "if else" statements that take the respective value based on the parameters defined. This part of the computation gives the components of $\mathrm{G}$ as defined earlier at every point of the Gaussian numerical integration and are stored in a row matrix of $\mathrm{N}$ columns for the sake of simplicity in defining the double summation used to solve for the components of $\mathrm{S}_{\mathrm{ijk} 1}$.

\subsubsection{Components of Eshelby's Tensor, $S_{\mathrm{ijkl}}$}

The term $\mathrm{S}_{\mathrm{ijkl}}$ represents a fourth order tensor which means it has 81 components that define the tensor completely. These components have to be computed individually depending upon the values that the indices $\mathrm{i}, \mathrm{j}, \mathrm{k}$ and 1 take (i.e. $\mathrm{i}=\mathrm{j}=\mathrm{k}=1=1,2,3$ ). These indices define the 81 components of $\mathrm{S}$ as $\mathrm{S}_{1111}, \mathrm{~S}_{1112}, \mathrm{~S}_{1213}$ and so on and are defined in the MATLAB code given in the appendix B. Solution for each individual component requires explicitly solving the eq. (2.6) for that component. This 
incorporates the values of the components of $\mathrm{G}$ that were derived in the previous section and the matrix of the gaussian weights defined in the inputs section. As all of the components are computed, they are stored in a matrix of order 9 as is shown in the appendix B and this matrix constitutes the Eshelby's tensor S.

\subsubsection{Mori-Tanaka calculations}

The Mori Tanaka method defined in the previous chapter is used to compute the effective properties of the composite material. The Eshelby's tensor calculated earlier forms the basis of the solution for the Mori-Tanaka method. In this section, a "for" loop is defined to incorporate the range of the volume fraction of the effective fiber over which the properties are determined. This loop solves the eq. (2.1) and (2.2), defined earlier, over the range of this fiber volume fraction to generate the elastic constants of the final composite material. The results are displayed in the command window of MATLAB.

\subsubsection{Combined processing}

Summarizing, the various sections defined earlier help in the computation of the effective properties of the composite. The accuracy of these calculations depends on the accuracy of user input to the program and can be modified as per the requirements of the final composite. Alteration in the definition of the shape and size of the fibers, the fiber volume fraction and the fiber orientation can be made to obtain properties corresponding to the applications of the final composite. Computation of the Eshelby's tensor needs to be done at each modification in the input, since $\mathrm{S}$ is vulnerable to any change in the properties defined here and is directly related to the accuracy of the final results. 


\subsubsection{Graphical Representation}

Representing the data obtained in a user friendly pattern is very important considering the large number of data points involved and the complexity in understanding the results from the command window directly. The last step in the program involved representing the data in a graphical form in order to the ease the comparison of properties obtained with different sets of modifications in the input properties. The various graphs obtained are shown and explained in the results section of this document. These contain different combinations of data points and incorporate all the variations in nanotube lengths, orientations and volume fractions. 


\section{Results and Discussion}

This section demonstrates the effective properties of the LCP/nanotube composite, derived from the research program, in a graphical and numerical form. The properties are a function of the nanotube volume fraction, nanotube length and the fiber orientation. In the computation of these properties, effective fiber volume was considered in order to account for the interphase conditions between the nanotubes and the polymer matrix and a nanotube volume fraction equivalent to $34 \%$ of the effective-fiber volume fraction was assumed based on the findings of Odegard et al. (G.M. Odegard et al. 2003). The effective properties calculated here consisted of the longitudinal Young's modulus, E1, the transverse Young's modulus, E2, and the longitudinal shear modulus, G12. These properties were computed for nanotube lengths ranging between $10 \mathrm{~nm}$ and $1000 \mathrm{~nm}$ for liquid crystal polymer composites with aligned and axisymmetrically oriented nanotubes. The orientations were randomly chosen between 0 and 90 degrees so that the behavior of the material could be studied.

It can be seen from Figure 4.1 that E1 depends, invariably, upon the length of the nanotubes used for reinforcement. Since, the longitudinal Young's modulus of the LCP is very small as compared to that of the nanotubes, the properties of the final composite are literally dependent on the nanotubes and are subject to change according to the variations in their length and orientation. It can be seen from the figure that there is a $560 \%$ increase in $\mathrm{E} 1$ for the composite with aligned nanotubes of length $500 \mathrm{~nm}$, with respect to the composite/LCP without reinforcement. The general trend that can be seen for nanotubes of all lengths is that the longitudinal Young's modulus increases with the increase in the nanotube volume fraction. This amount of increase, here, is directly proportional to the increase in length of the nanotubes. However, it is of importance to note that this increase in the longitudinal Young's 
modulus of the composite is experienced only until nanotubes of length $300 \mathrm{~nm}$ are used.

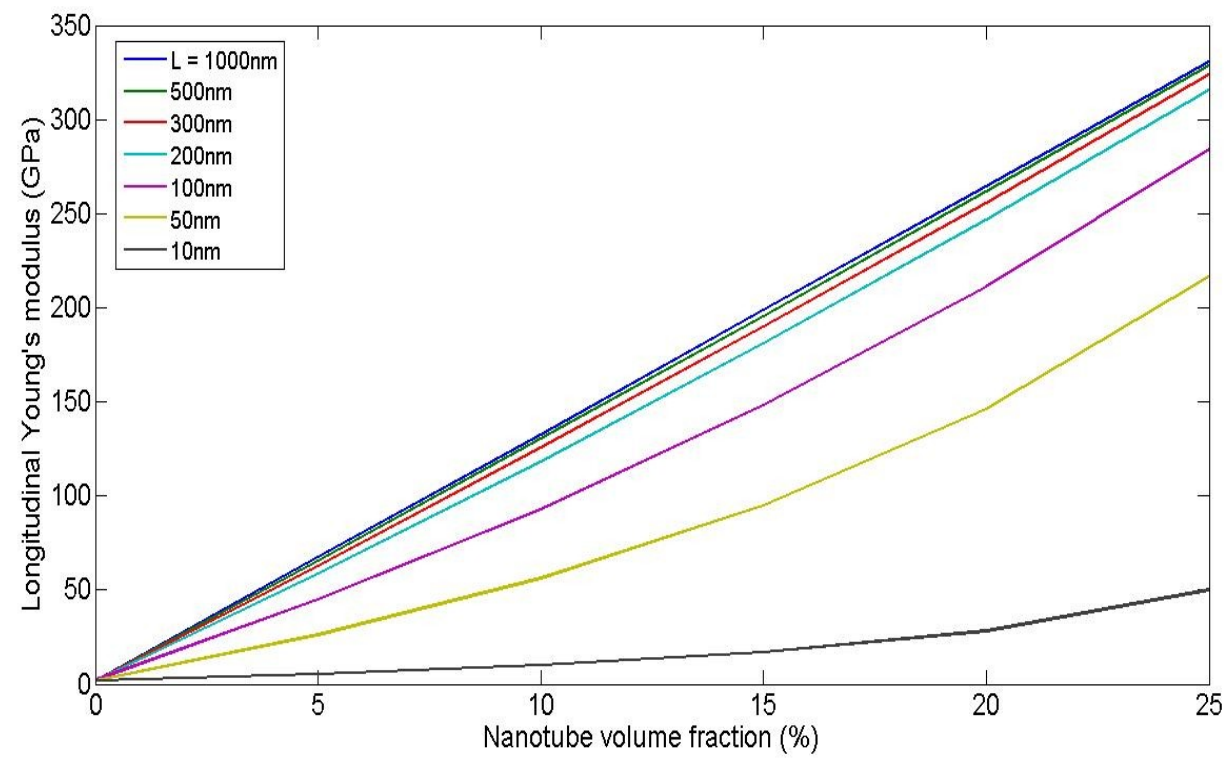

Figure 4.1: Graph showing longitudinal Young's modulus (E1) plotted v/s the nanotube volume fraction (vf) for unidirectionally aligned fibers of various lengths.

It can be seen that even at low nanotube concentrations, exceptional results in the longitudinal Young's modulus are obtained along all lengths of the nanotubes. This may be because of the superiority of the material properties of the nanotubes over those of the LCP. Not much improvement in the longitudinal Young's modulus is observed with nanotube lengths more than $300 \mathrm{~nm}$. This can be seen from the graph where the lines showing the longitudinal Young's modulus for nanotube lengths of $500 \mathrm{~nm}$ and $1000 \mathrm{~nm}$ are fairly close to each other or are nearly coinciding. A better view of the results can be obtained from the Figure 4.2 where the graph has been zoomed in to show results over the nanotube volume fractions from 0 to $5 \%$. The relation between the longitudinal Young's modulus and the volume fraction is directly related to the length of the nanotubes and increases with the increase in length. This can be attributed to the effect of increase in the load transfer to the 
nanotubes with increase in their concentration (H. Fukudu and Y. Takao 2000). To summarize, increase in moduli value is directly proportional to the increase in nanotube volume fraction.

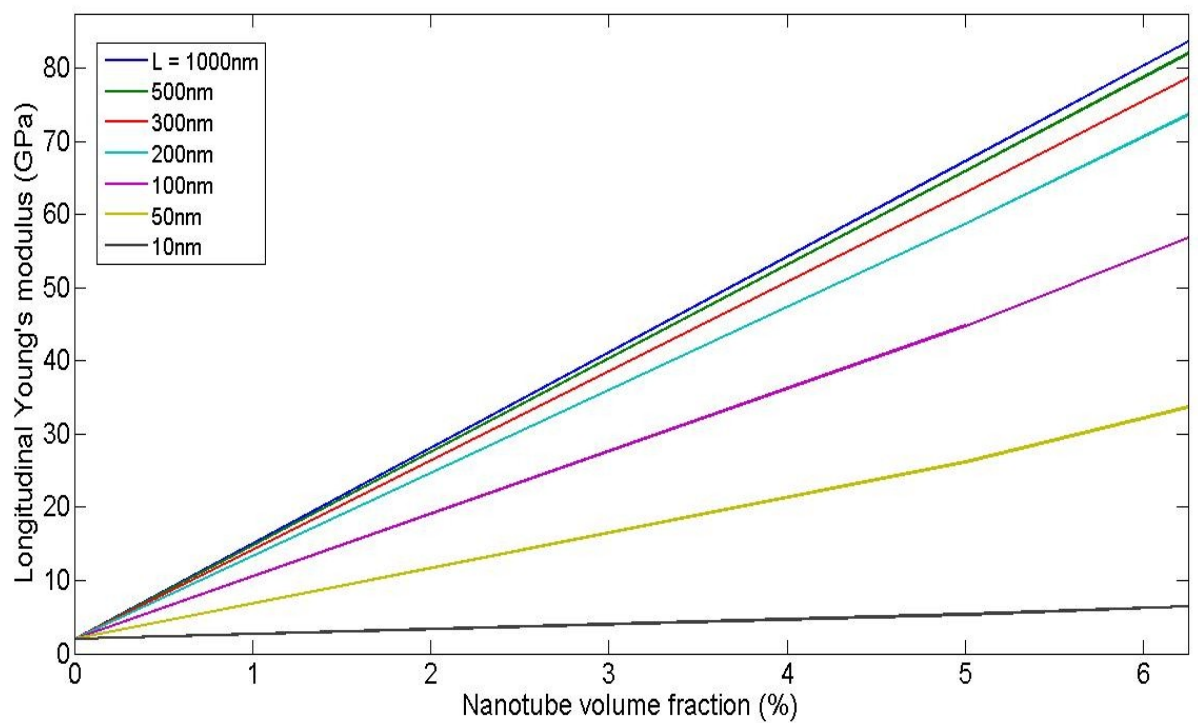

Figure 4.2: Graph showing longitudinal Young's modulus (E1) plotted v/s the nanotube volume fraction (vf) for unidirectionally aligned fibers of various lengths at volume fractions ranging from 0 to $5 \%$

Figure 4.3 shows the transverse Young's modulus of the composite plotted against the nanotube volume fraction for various fiber lengths. The data indicates that although the transverse modulus increases with the increase in the fiber volume fraction, the length of nanotubes doesn't seem to have any significant effect upon its magnitude. This may be because the transverse modulus is a matrix dependent property and, thus, is unaffected by the change in length of the nanotubes (Daniel and Ishai 1994). There is an increase of $33.33 \%$ in the transverse Young's modulus at 5\% nanotube volume fraction from when no reinforcement is used at all which is evident in the data obtained. This property of the composite of producing a high Young's modulus in one direction and a very low modulus in the other can be used in 
applications where directional stiffness plays an important role in the properties of a component. It is important to note that the graph of increase in the transverse modulus as per the increase in the nanotube volume fraction is mostly linear for all lengths of the nanotube. This is unlike the graph for longitudinal modulus which, as the length of nanotubes increase, becomes linear, but shows no linearity at smaller lengths.

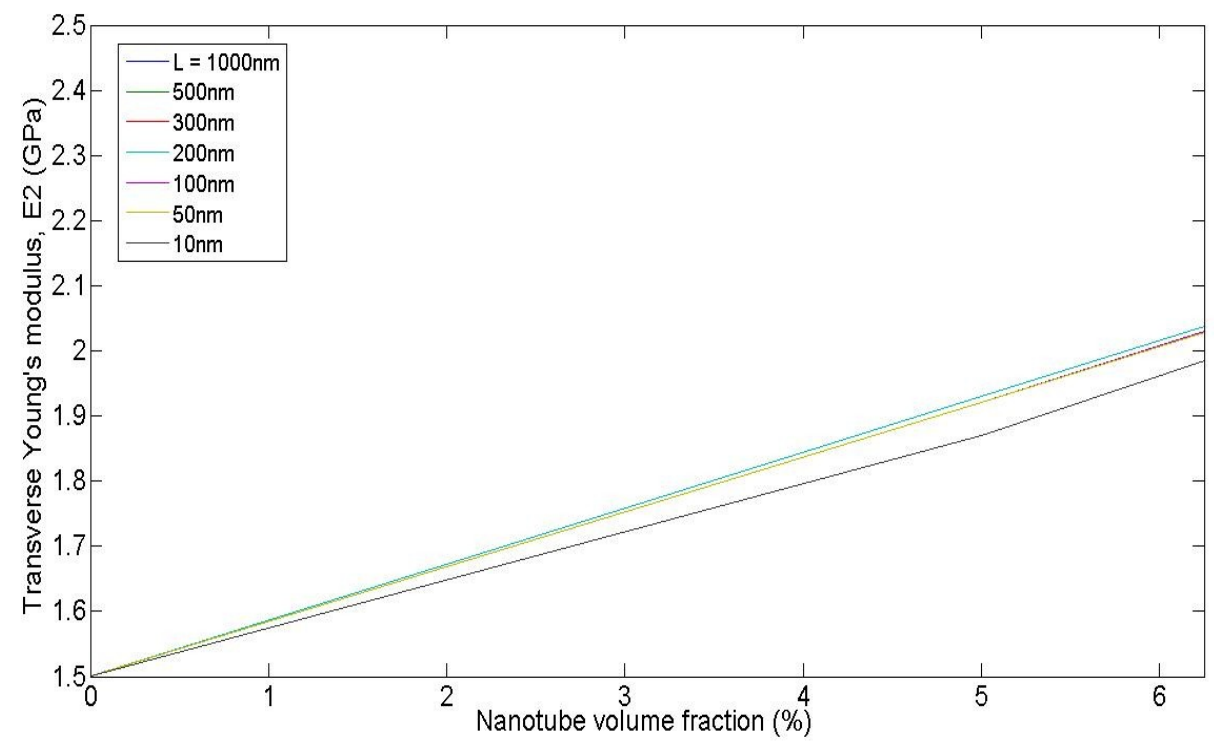

Figure 4.3: Graph showing transverse Young's modulus (E2) plotted v/s the nanotube volume fraction (vf) for unidirectionally aligned fibers of various lengths at volume fractions ranging from 0 to $5 \%$

Figure 4.4 is a graph of longitudinal shear modulus (G12) plotted against the nanotube volume fraction for various lengths of the nanotube. Since the values of the modulus do not change significantly according to the change in length of the nanotubes, the graph lines for some lengths coincide and are not clearly visibile. In general, an increase in the nanotube volume fraction resulted in the increase in longitudinal shear modulus of the nanocomposite. Only changes in the shear modulus relating to changes in the length of the nanotubes were visible above a nanotube volume fraction of around $10 \%$. It can be inferred that the shear strength of the 
$\mathrm{LCP} /$ nanotube composite is much less as compared to its longitudinal strength at a $25 \%$ nanotube volume fraction. Thus, LCP/nanotube composite shows high stiffness in the axial direction for unidirectionally aligned nanotubes at 0 degrees.

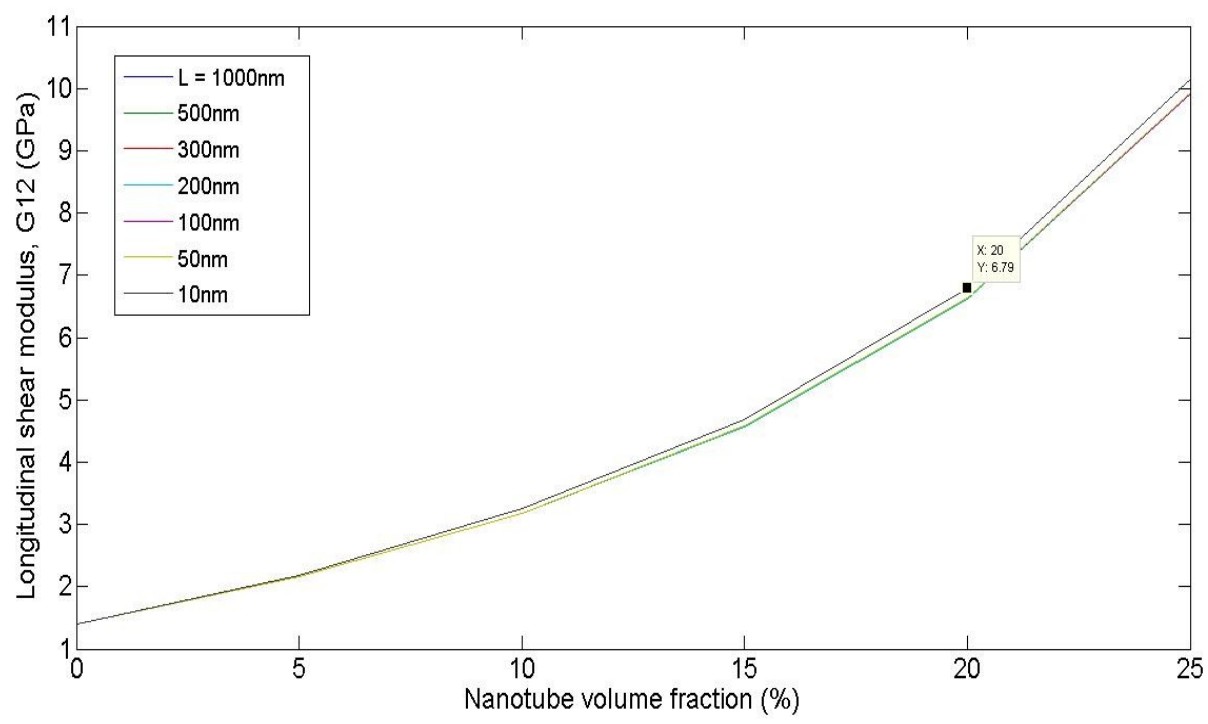

Figure 4.4: Graph showing longitudinal shear modulus (G12) plotted v/s the nanotube volume fraction (vf) for unidirectionally aligned fibers of various lengths.

Figure 4.5 shows a graph of longitudinal Young's modulus (E1) plotted against the nanotube volume fraction for different orientations of the nanotubes aligned unidirectionally. It can be seen that there is a significant drop in the modulus even for a 15 degree orientation of the nanotubes as related to the matrix molecules. This drop in modulus is noted to be around $65 \%$. This percentage amount decrease in the modulus is a significant amount and is because of the dependency of E1 entirely on the longitudinal Young's modulus of the nanotubes. The modulus value for the LCP is negligible as compared to that of the nanotubes. As the nanotubes are further oriented until they are aligned in a direction perpendicular to the direction of the LCP molecules the modulus goes on decreasing and is found to be lowest at 90 degree 
orientation. Once the 45 degree mark is crossed no significant change in E1 is noted. Similar plots are obtained for E2 and G12 and are shown in the figures below.

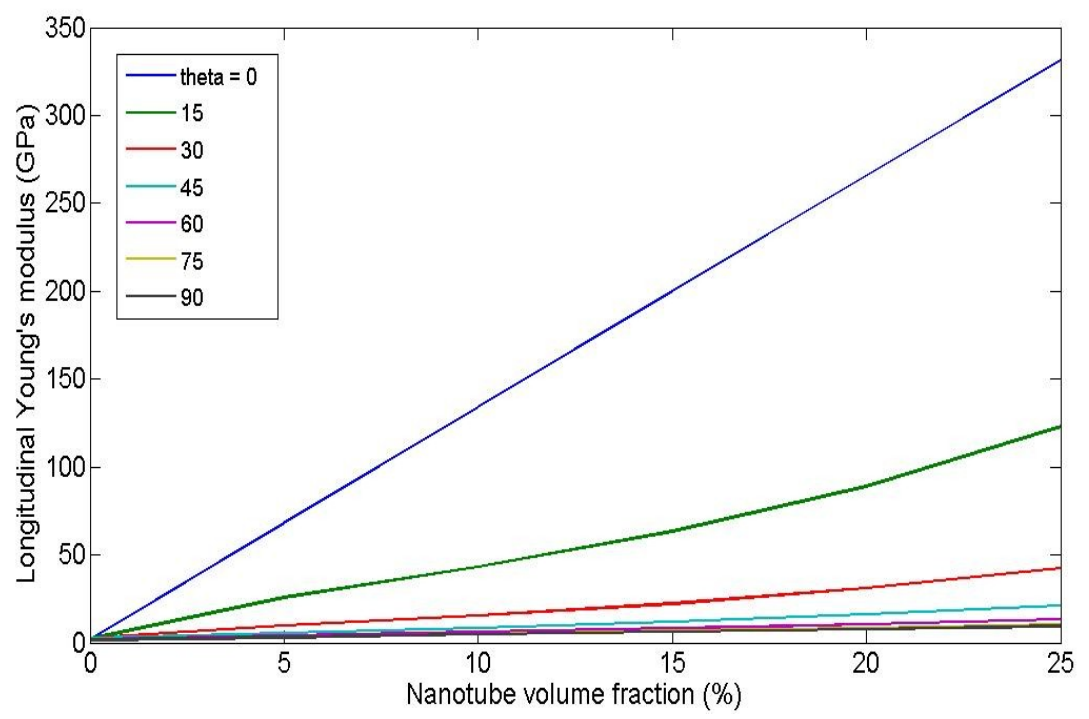

Figure 4.5: Graph showing Longitudinal Young's modulus (E1) plotted against the nanotube volume fraction (vf $0-5 \%$ ) for various orientations of the the nanotubes at the length of $500 \mathrm{~nm}$.

It can be seen from Figure 4.6 that as the nanotubes are oriented with reference to the longitudinal axis of the composite, the longitudinal Young's modulus decreases whereas; the transverse Young's modulus increases. This, again, is because of the dependency of the modulus on the properties of nanotubes. On the other hand, Figure 4.7 shows that the longitudinal shear modulus at 5\% nanotube volume content is greater when nanotubes are aligned in the same direction as that of LCP's than when they are oriented at 90 degrees. Whereas, this is vice-versa at very low nanotube volume content such as $1 \%$.

Longitudinal Young's modulus is plotted against the length $(\mathrm{nm})$ of nanotubes at various orientations in Figure 4.8. It is to be noted here that, at 0 degree orientation, there is no steep rise in the modulus after a length of around $300 \mathrm{~nm}$. This is also 
evident from Figure 4.1 where the curves for modulus at $500 \mathrm{~nm}$ and $1000 \mathrm{~nm}$ nearly coincide. This might be because the load transfer to the nanotubes depends vastly upon their aspect ratio and since this ratio for $500 \mathrm{~nm}$ and $1000 \mathrm{~nm}$ nears infinity, no significant effect is seen as far as E1 is concerned. It can be said that the $1000 \mathrm{~nm}$ nanotubes are much more flexible as compared to the $500 \mathrm{~nm}$ ones and are subject to fail earlier.

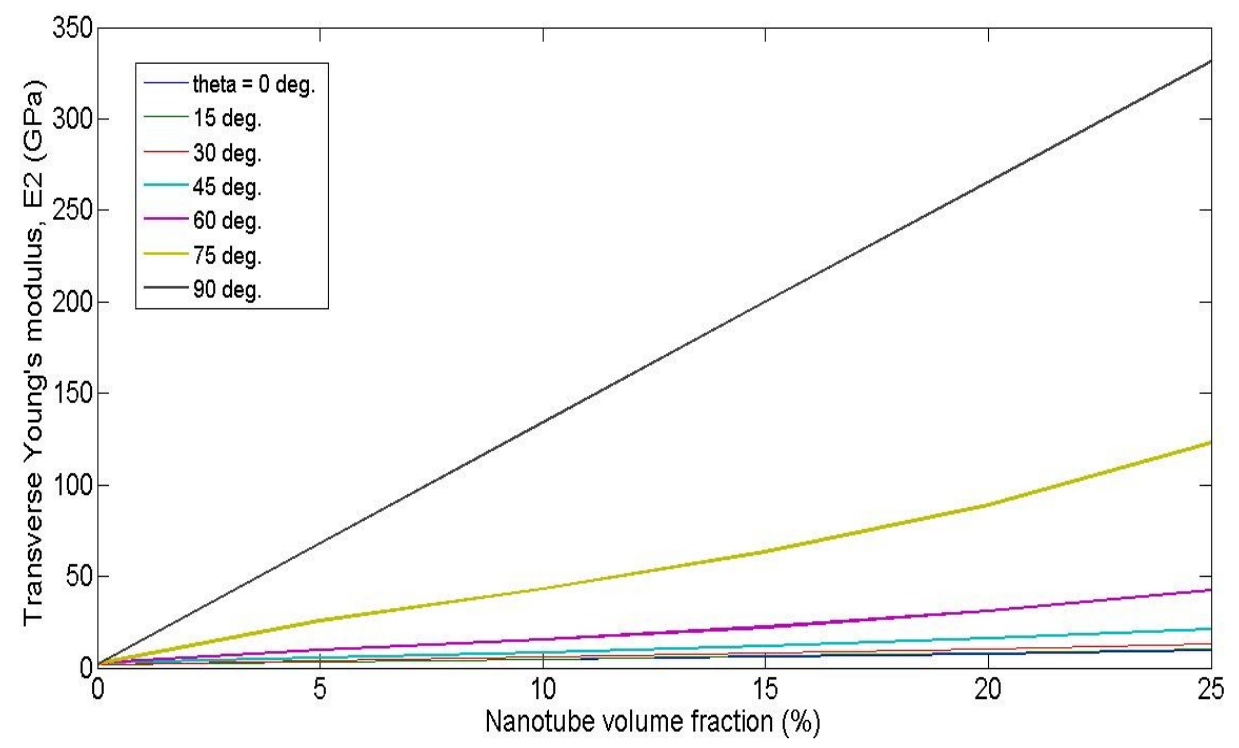

Figure 4.6: Graph showing transverse Young's modulus (E2) plotted against the nanotube volume fraction (vf $0-5 \%$ ) for various orientations of the the nanotubes at the length of $500 \mathrm{~nm}$.

The curves for E2 for the same case as above are exactly opposite to those of E1. Since, the nanotubes experience orientation up to 90 degrees, E2 also achieves magnitude from the lowest at 0 degree to the highest at 90 degrees which equals E1 at 0 degree nanotube orientation. This can be seen in Figure 4.9 where magnitudes of E2 are plotted against the nanotube lengths. At 0 degrees though, the magnitude of E2 does not change with the change in the aspect ratio of the nanotubes since, the transverse Young's modulus is a matrix dependent property (Daniel and Ishai 1994). 
Figure 4.10 is a plot of longitudinal shear modulus plotted against the nanotube length for various orientations of the nanotubes at $5 \%$ volume fraction.

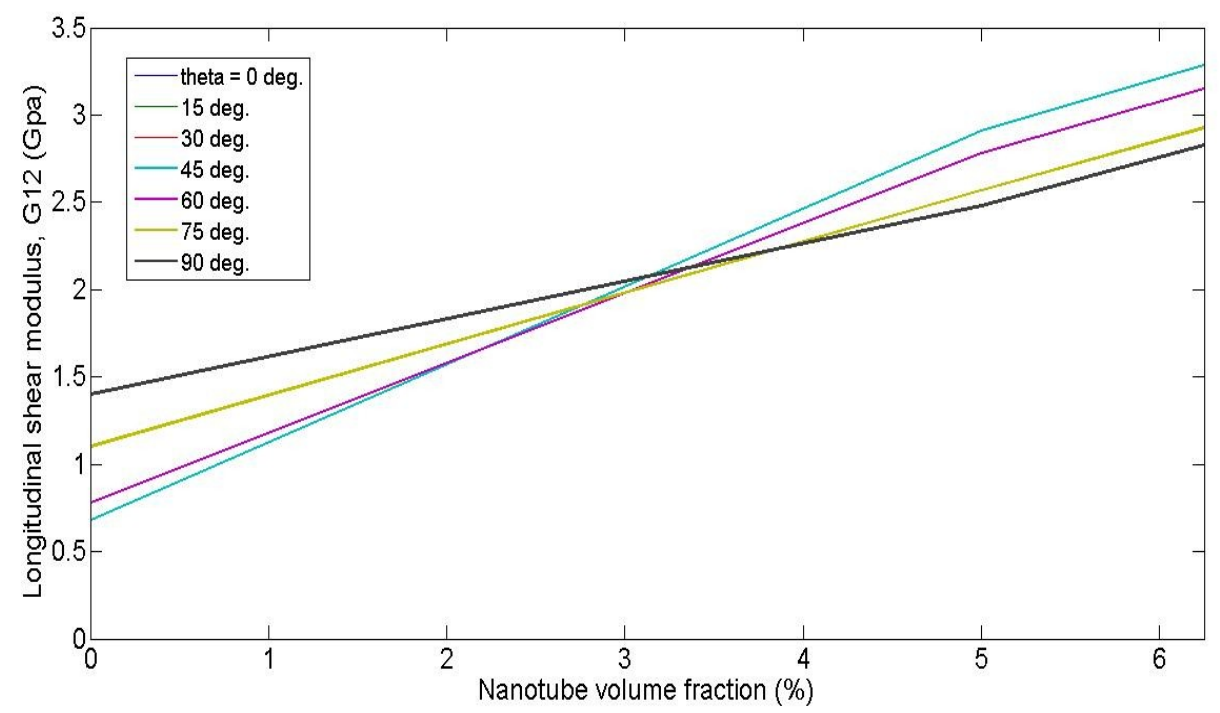

Figure 4.7: Graph showing longitudinal shear modulus (G12) plotted against the nanotube volume fraction (vf $0-5 \%$ ) for various orientations of the the nanotubes at the length of $500 \mathrm{~nm}$. 


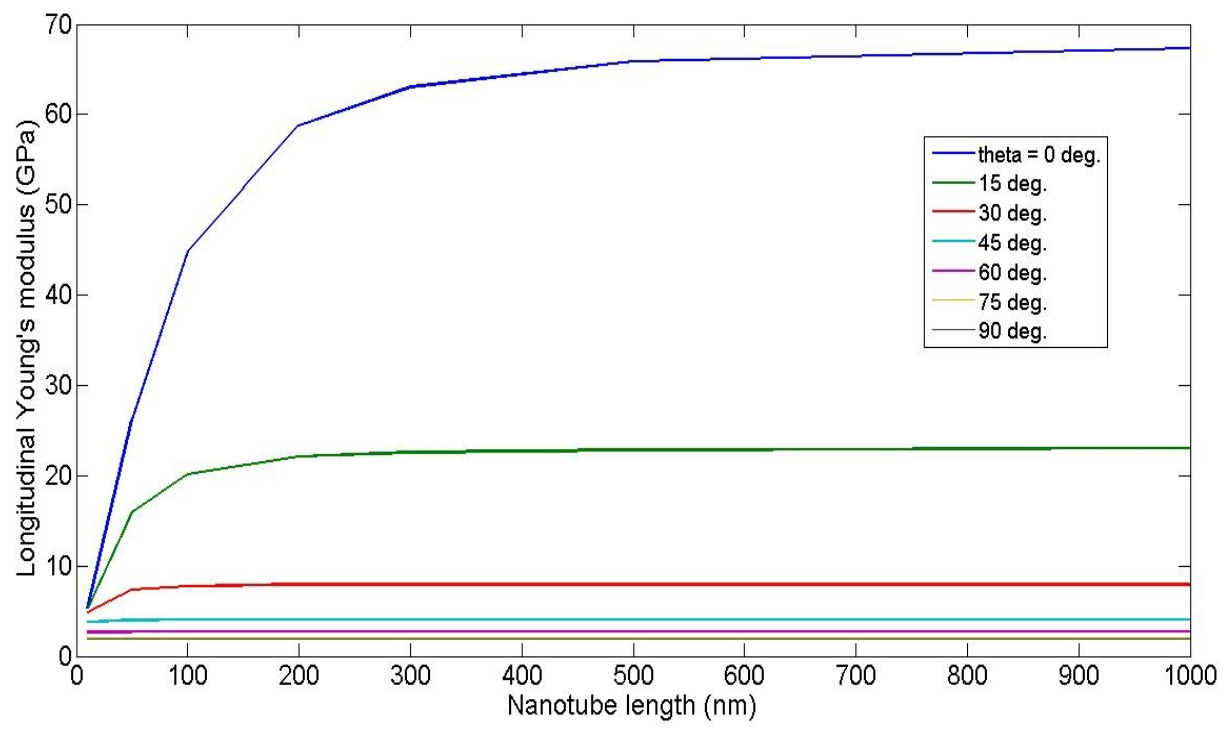

Figure 4.8: Graph showing Longitudinal Young's modulus (E1) plotted against the nanotube length $(\mathrm{nm})$ at a $5 \%$ nanotube volume fraction for various orientations of the the nanotubes.

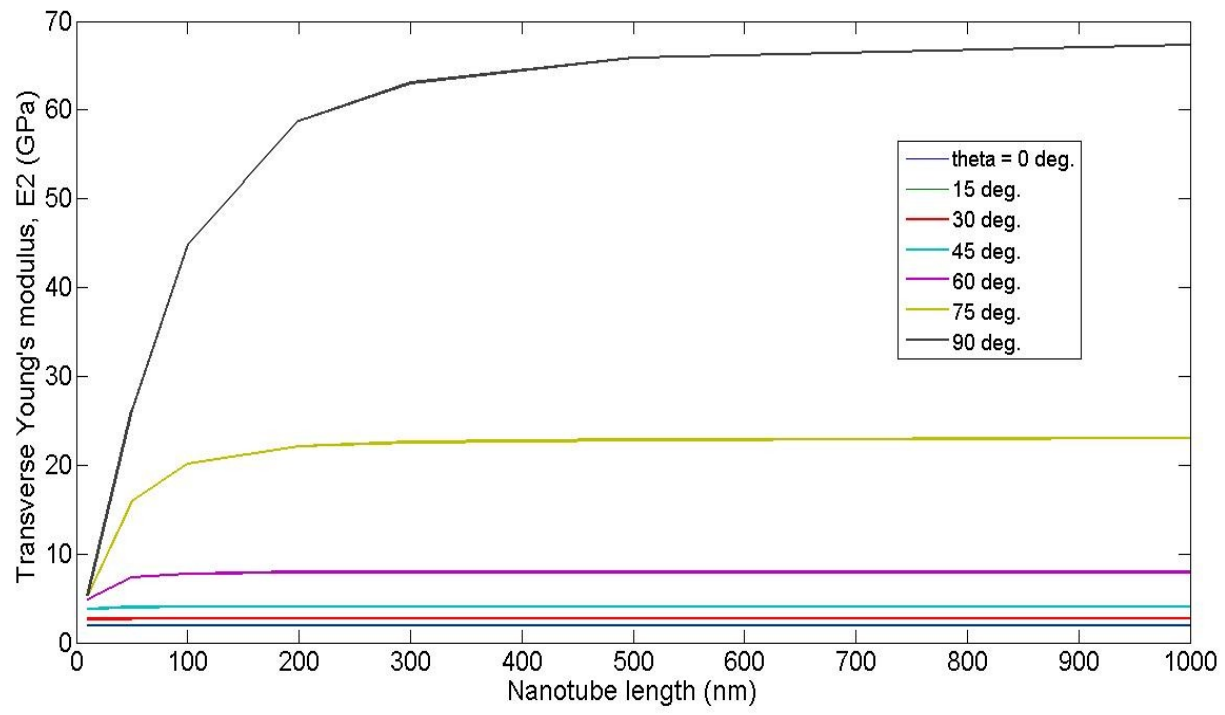

Figure 4.9: Graph showing transverse Young's modulus (E2) plotted against the nanotube length $(\mathrm{nm})$ at a $5 \%$ nanotube volume fraction for various orientations of the the nanotubes 


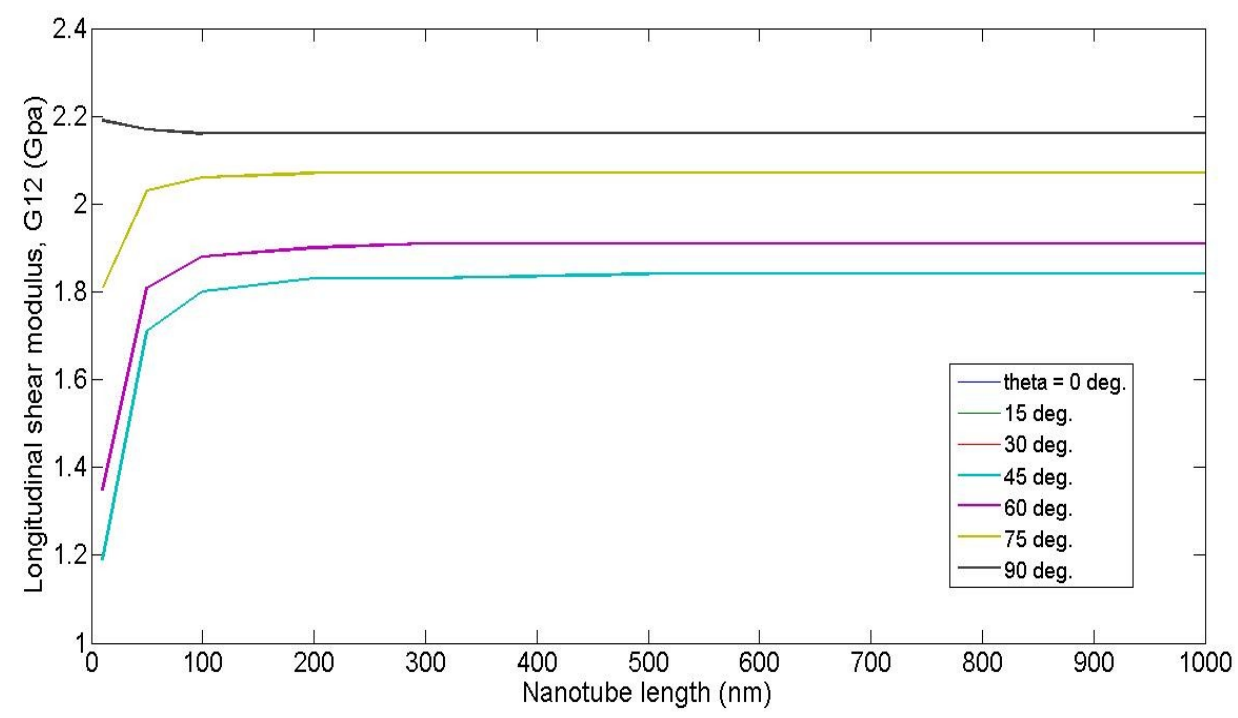

Figure 4.10: Graph showing longitudinal shear modulus (G12) plotted against the nanotube length $(\mathrm{nm})$ at $5 \%$ nanotube volume fraction for various orientations of the the nanotubes.

Figure 4.10 is interesting because of the pattern experienced in the values of G12 along all the orientations. Irrespective of the angle at which the nanotubes are aligned as compared to the LCPs, the magnitude of G12 tends to remain constant over a nanotube length of $100 \mathrm{~nm}$. A very steep increase in the magnitude is observed for 45 degrees orientation of the nanotubes from a length of $1 \mathrm{~nm}$ to $100 \mathrm{~nm}$ and is recorded to be around $50 \%$. 


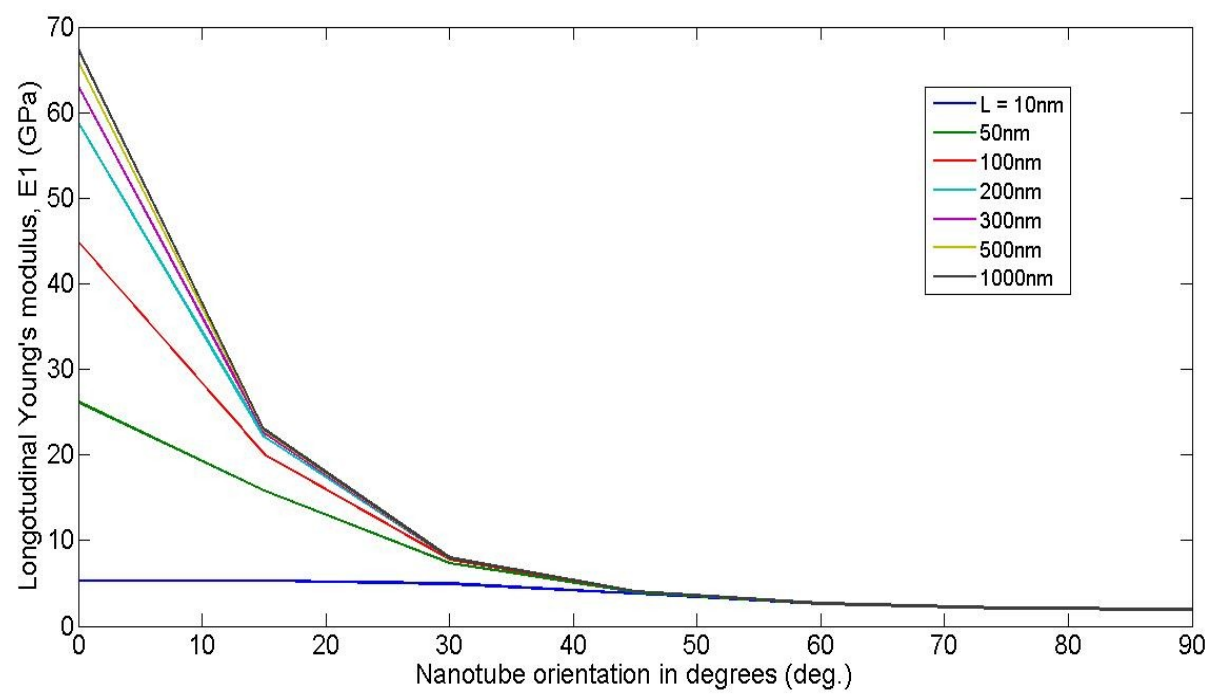

Figure 4.11: Graph showing Longitudinal Young's modulus (E1) plotted against the nanotube orientation (deg.) for various lengths of the nanotubes at 5\% nanotube volume content.

Longitudinal Young's modulus has been plotted against the nanotube orientation, for various lengths of the nanotubes, in Figure 4.11 at a $5 \%$ nanotube volume fraction. For all the nanotube lengths the modulus decreases with the increase in the nanotube orientation angle. This is similar to the data in Figure 4.5 and also is because of the dependency of the longitudinal modulus on nanotube properties. For nanotubes that are $500 \mathrm{~nm}$ or even $1000 \mathrm{~nm}$ long, the drop in modulus values with increase in orientation is significant and can be seen in the figure. For nanotubes that are $500 \mathrm{~nm}$ long, the drop in modulus with orientation increase from 0 to 90 degrees is approximately $96.3 \%$. 


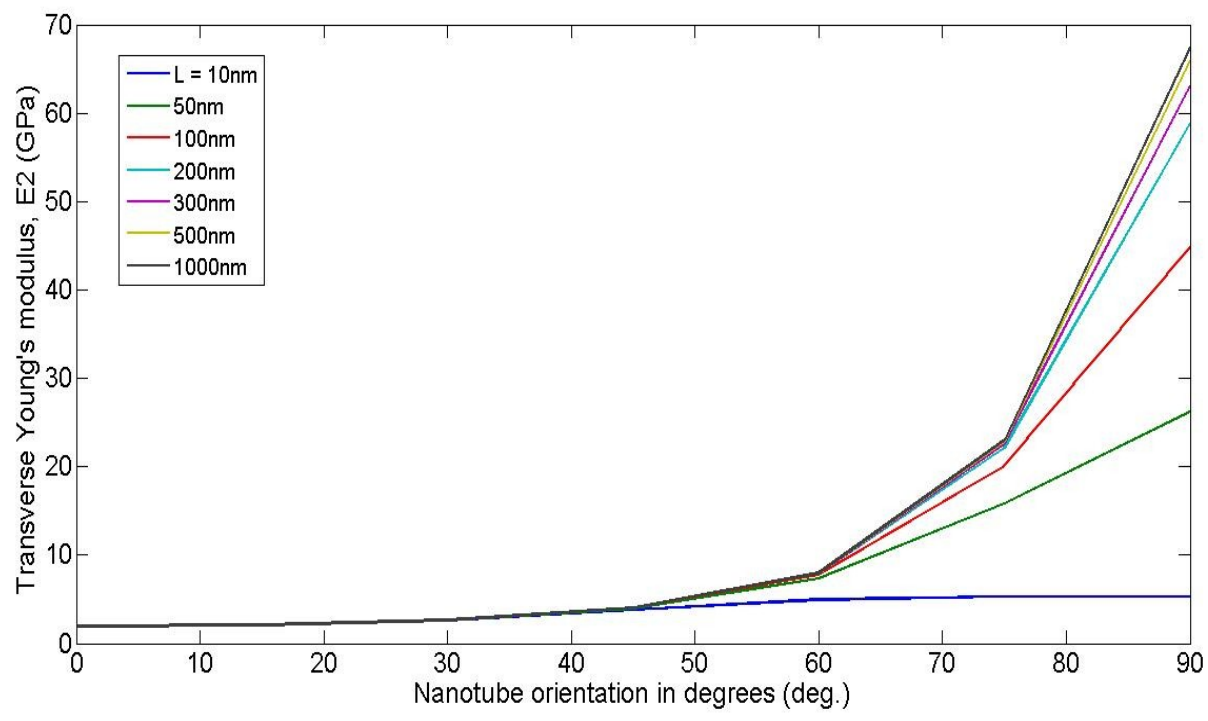

Figure 4.12: Graph showing transverse Young's modulus (E2) plotted against the nanotube orientation (deg.) for various lengths of the nanotubes at 5\% nanotube volume content.

Again, just like the Figures 4.8 and 4.9, the graphs shown by Figures 4.11 and 4.12 denote curves that represent exactly opposite data. As the nanotubes are oriented with respect to the longitudinal axis of the composite, the longitudinal Young's modulus decreases and the transverse Young's modulus increases and at 90 degrees orientation the transverse Young's modulus is equal to the longitudinal Young's modulus at a 0 degree orientation. The longitudinal shear modulus, for the same case as above, has been plotted against the nanotube orientation for various lengths of the nanotubes and is shown in Figure 4.13. It can be noted that for all lengths of the nanotubes used, G12 was lowest at 45 degrees orientation of the nanotubes and highest at 0 and 90 degrees orientations. The steepest drop at the 45 degree orientation is with nanotubes that are $10 \mathrm{~nm}$ long while the lowest drop is with nanotubes that are $1000 \mathrm{~nm}$ long. There is, again, a big drop in magnitude of G12 while nanotubes are switched from $50 \mathrm{~nm}$ length to $10 \mathrm{~nm}$ length which signifies that the shorter the length of nanotubes 
used, higher is the effect of orientation and volume fraction on the final properties of the composite, since the properties mainly depend upon the nanotubes.

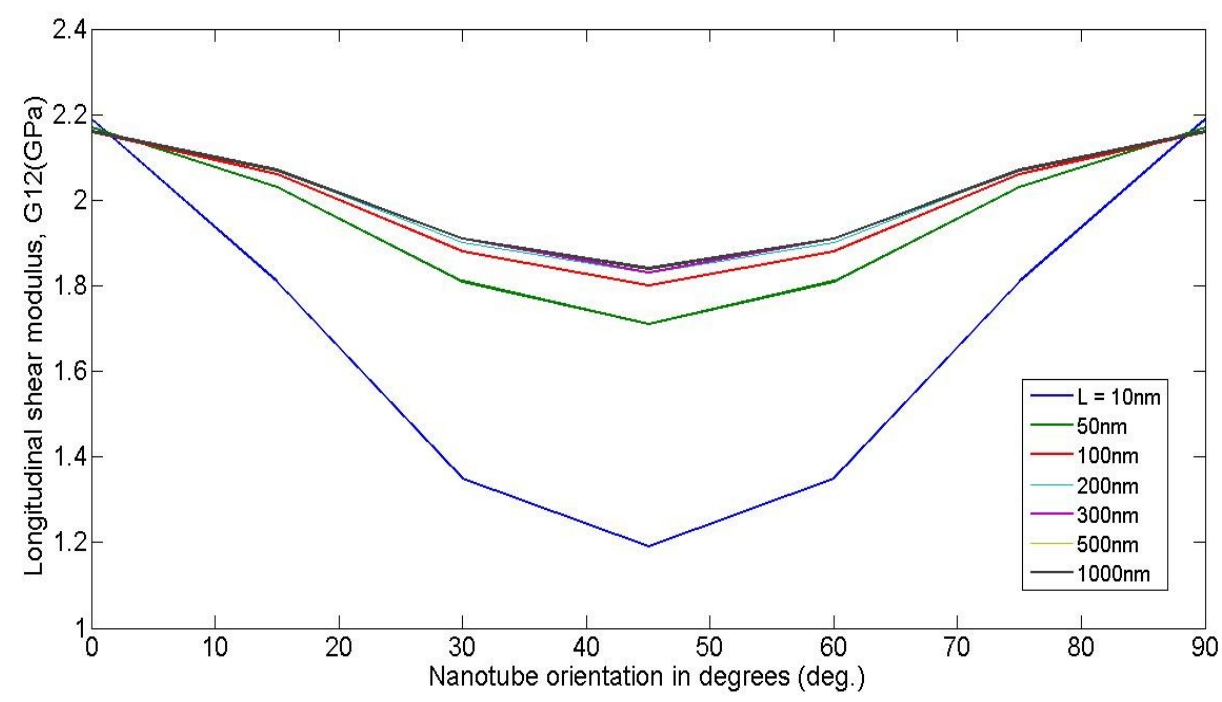

Figure 4.13: Graph showing longitudinal shear modulus (G12) plotted against the nanotube orientation (deg.) for various lengths of the nanotubes at $5 \%$ nanotube volume content.

Figure 4.14 plots the longitudinal Young's modulus against the orientation of nanotubes that are $500 \mathrm{~nm}$ long for varying nanotube content (volume). The nanotube volume fraction affects the longitudinal Young's modulus until the orientation of around 45 degrees after which the graph lines almost tend to straighten out and coincide with each other. The orientation has no more effect on the magnitude of the modulus. For a nanotube volume content of 5\% from 0 to 45 degrees orientation, the drop in the longitudinal Young's modulus is recorded to be around 95\%. From about 60 to 90 degrees orientation, the nanotube volume fraction does not seem to play a role in determining the longitudinal modulus of the composite. 


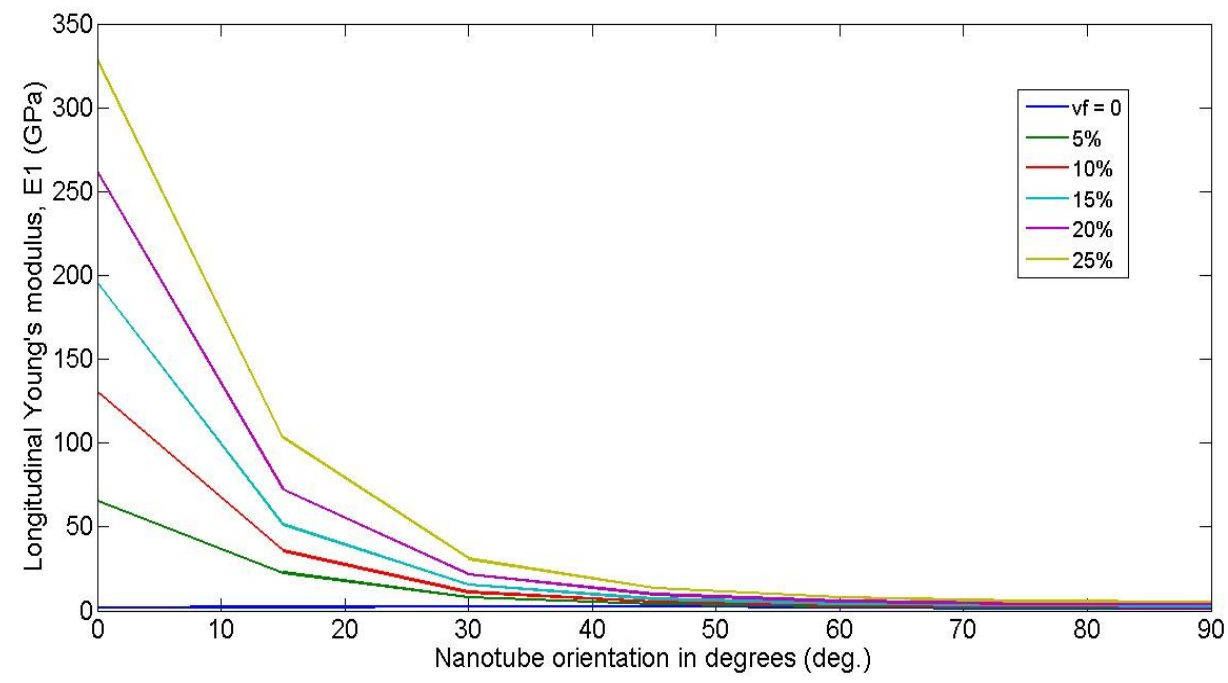

Figure 4.14: Graph showing longitudinal Young's modulus (E1) plotted against the nanotube orientation (deg.) for varying volume fractions of nanotubes of length $500 \mathrm{~nm}$.

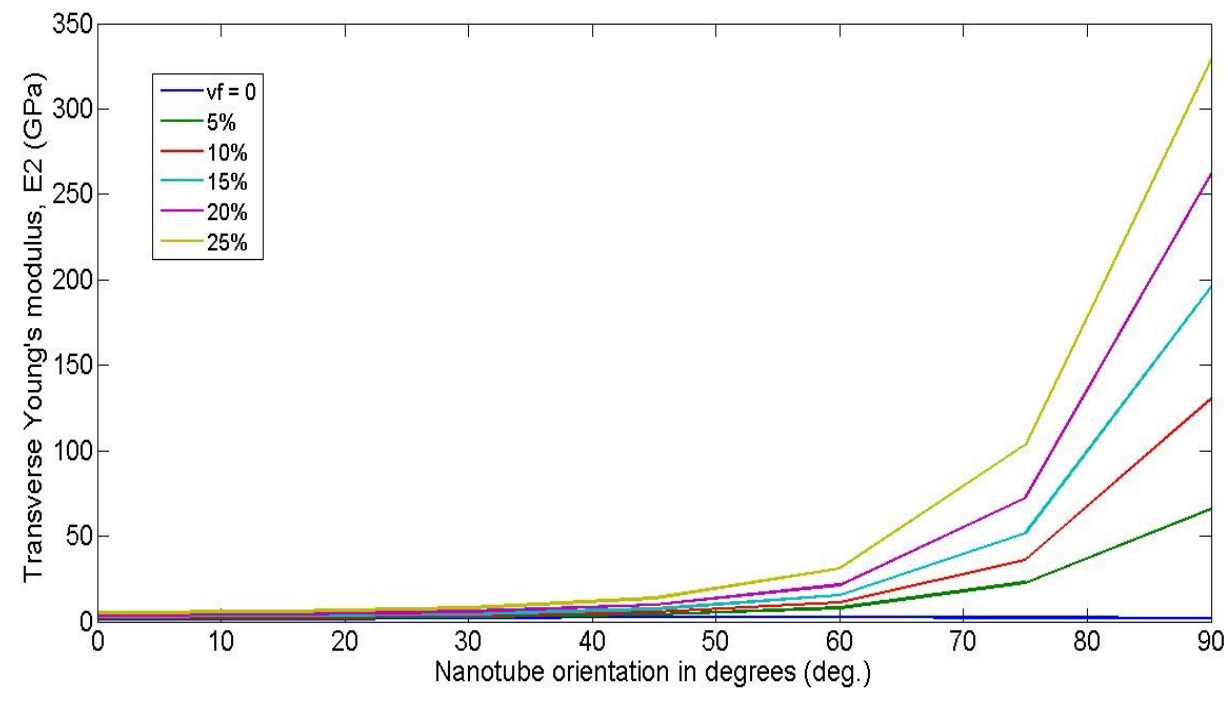

Figure 4.15: Graph showing transverse Young's modulus (E2) plotted against the nanotube orientation (deg.) for varying volume fractions of nanotubes of length $500 \mathrm{~nm}$. 
Again, Figure 4.15 is similar to Figure 4.14 only that the data is plotted in the exact opposite manner. Here, since transverse modulus is being measured, the magnitudes start escalating once the nanotube orientation reaches and crosses 45 degrees. As the nanotubes draw closer to the transverse axis of the composite, the magnitude almost reaches and at 90 degrees, finally, becomes equal to the longitudinal modulus of the composite at 0 degree orientation. Also, from 0 to 45 degrees orientation, the nanotube volume fraction does not seem to affect the transverse Young's modulus at all. The steep rise in the modulus starts only after the orientation of 45 degrees. It can be seen that even the slightest concentration of nanotubes tends to largely increase the modulus of the composite and is most efficient for E1 when nanotubes are aligned uniaxially with the LCP molecules and for E2 when they are aligned in a perpendicular direction.

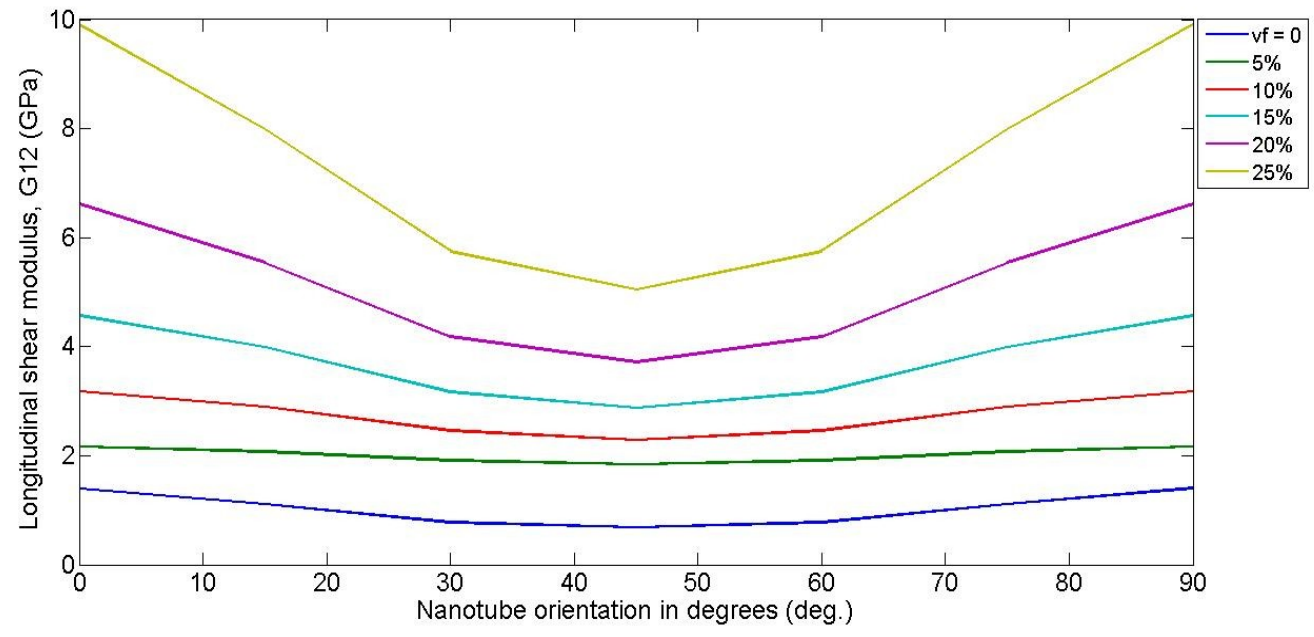

Figure 4.16: Graph showing longitudinal shear modulus (G12) plotted against the nanotube orientation (deg.) for varying volume fractions of nanotubes of length $500 \mathrm{~nm}$.

The longitudinal shear modulus (G12) has been plotted against various nanotube orientations for varying volume contents of the nanotubes that are $500 \mathrm{~nm}$ long and is 
shown in the Figure 4.16. Again, just like in Figure 4.13, the graph is symmetric about a vertical axis drawn at the 45 degree mark. For no nanotube concentration, the magnitude of the shear modulus does not vary too much as per the orientation. However, as the nanotube volume content increases, the orientation has greater effects upon the magnitude of the modulus. The shear modulus has the highest magnitude at 0 and 90 degree orientations for $25 \%$ nanotube volume content.

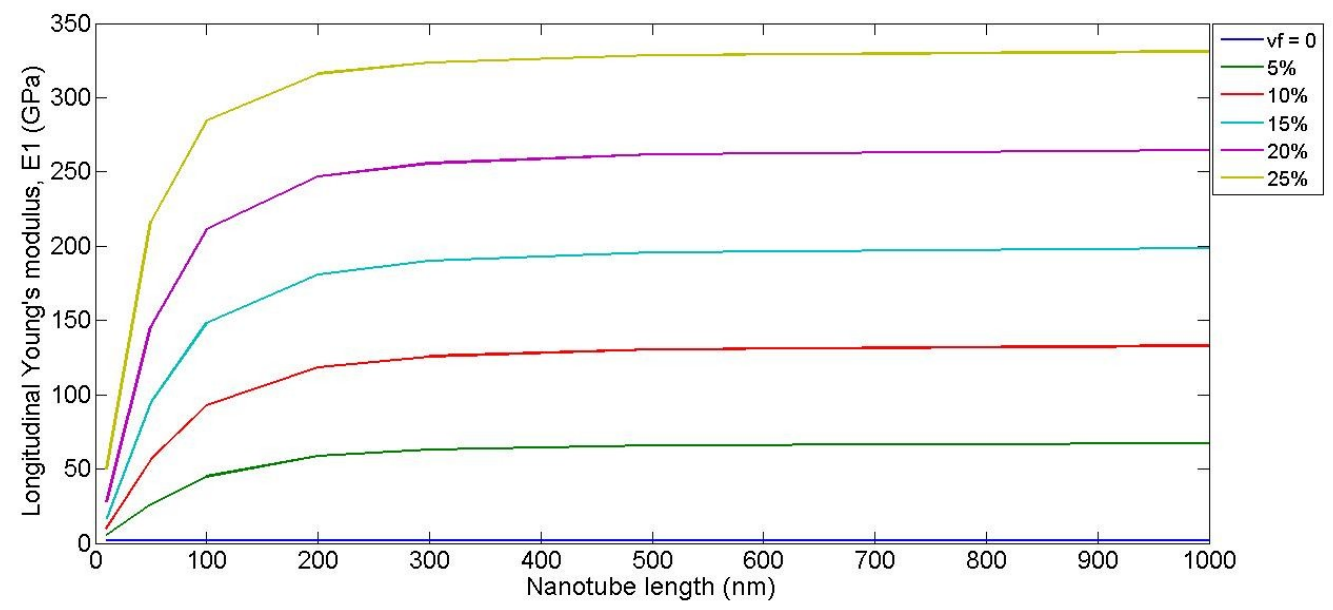

Figure 4.17: Graph showing longitudinal Young's modulus (E1) plotted against the nanotube lengths $(\mathrm{nm})$ for varying volume fractions of nanotubes aligned uniaxially with the LCP molecules.

Figures 4.17 and 4.18 plot the longitudinal and transverse Young's modulus of the composite against the nanotube lengths and is plotted for various nanotube volume contents that are aligned uniaxially with the LCP monomers. Thus, the plot does not take nanotube orientation into consideration. It is worthwhile to note that nanotube length has a significant effect on the longitudinal modulus of the composite whereas the transverse modulus does not vary at any point in time. This is because of the dependency of the transverse modulus on the properties of the matrix material (Daniel and Ishai 1994) although, it can be seen from previous figures that orientation of the nanotubes does have a significant effect upon the transverse modulus of the material. 
From Figure 4.17 it can be seen that, at 25\% nanotube volume content, no considerable change is observed in the longitudinal Young's modulus if nanotubes above a length of about $300 \mathrm{~nm}$ are used. This is also evident from Figure 4.1 where the curves for nanotube lengths $500 \mathrm{~nm}$ and $1000 \mathrm{~nm}$, for the longitudinal Young's modulus, almost seem to coincide with each other. At a nanotube length of $300 \mathrm{~nm}$, there is approximately a $540 \%$ increase in the modulus as the nanotube volume content increases from $5 \%$ to $25 \%$. Thus, it can be said that even the slightest concentration of the nanotubes increases the stiffness significantly in the longitudinal direction of the composite whereas the stiffness in the transverse direction does not change at all. Although the length of nanotubes does not affect the transverse Young's modulus, the nanotube volume fraction affects it and the modulus increases with the increase in the nanotube concentration and can be seen in Figure 4.18 where for a nanotube concentration increase from $5 \%$ to $25 \%$, the transverse Young's modulus increases by about $175 \%$ and is almost the same for all lengths of the nanotubes used.

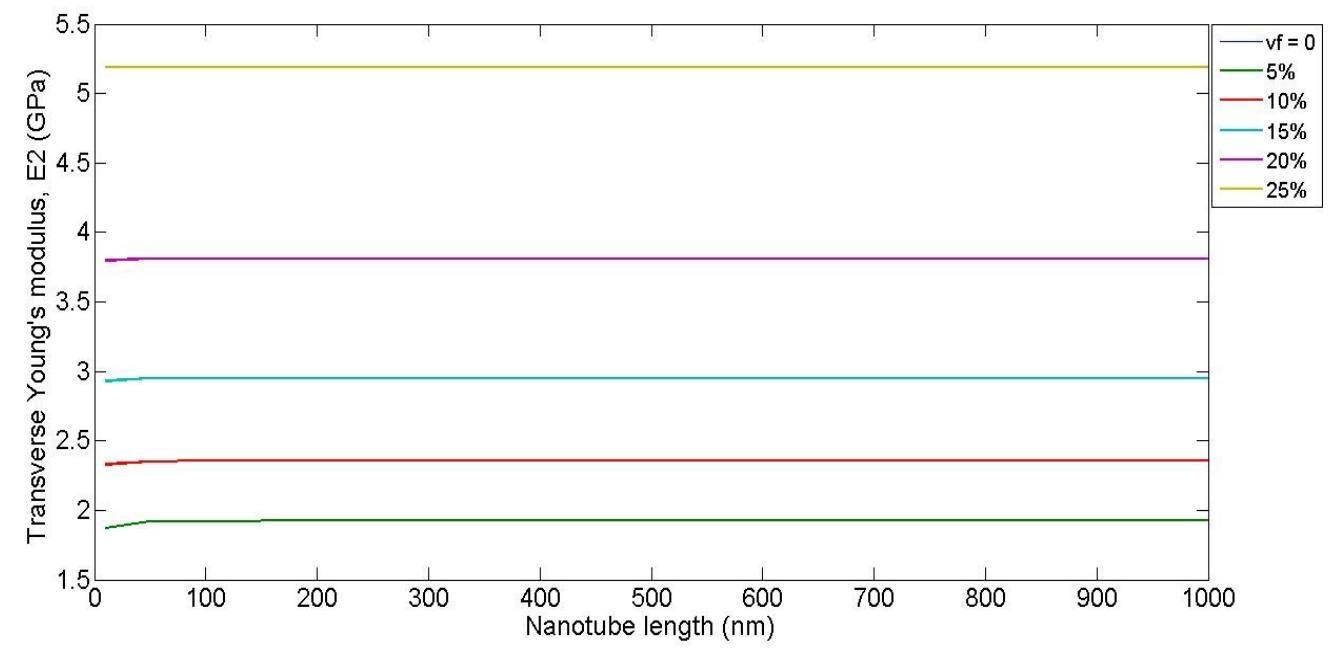

Figure 4.18: Graph showing transverse Young's modulus (E2) plotted against the nanotube lengths $(\mathrm{nm})$ for varying volume fractions of nanotubes aligned uniaxially with the LCP molecules. 
Figure 4.19 suggests that the longitudinal shear modulus, just as the transverse Young's modulus in Figure 4.18, does not seem to change according to the change in length of the nanotubes used. Although, a slight change in the modulus values is noted at lengths of $10 \mathrm{~nm}$ and $50 \mathrm{~nm}$ for $20 \%$ and $25 \%$ fiber volume content, it is not considerable and can be neglected. As the nanotube volume content increases from $0 \%$, the modulus value also increases and is the same for all the lengths of the nanotubes used. For a change of nanotube volume content from 0 to $25 \%$, the shear modulus increases by about $615 \%$. Thus, it is to be noted that the concentration of nanotubes does affect the longitudinal shear modulus of the composite material. This can be explained by saying that the increase in the shear modulus values is the effect of the increase in resistance of the material to shearing strains due to the extremely high modulus values of the nanotubes.

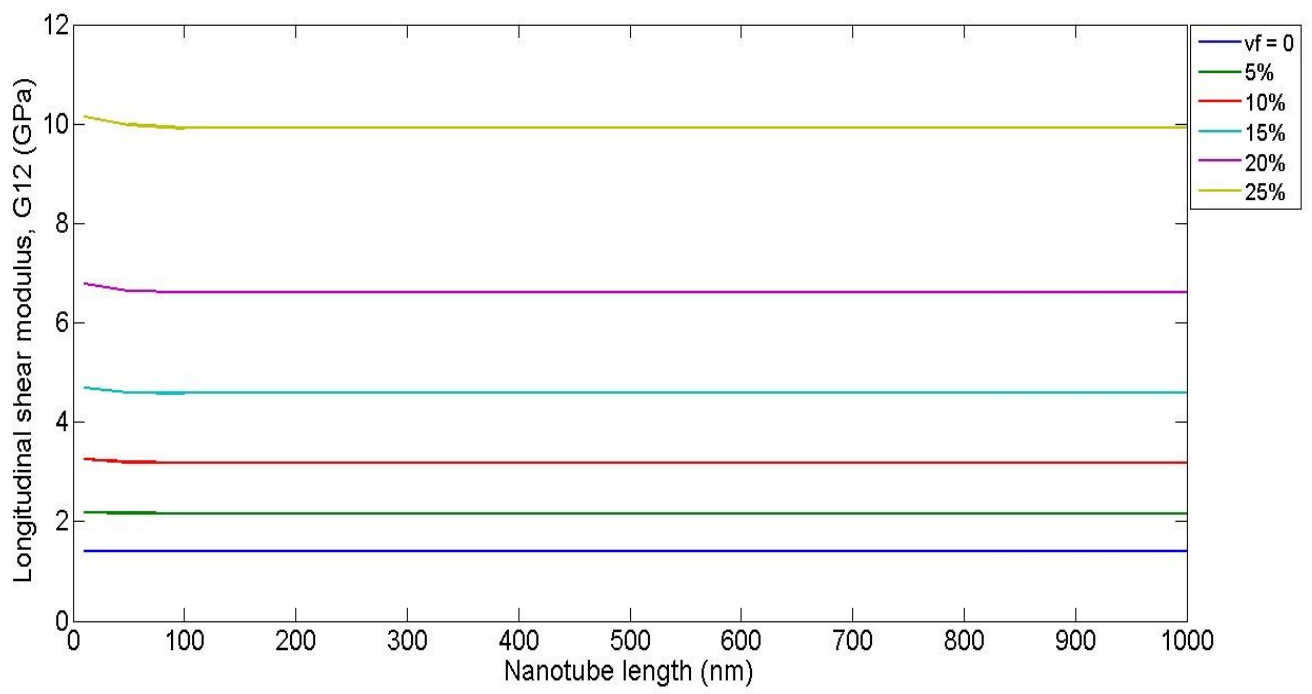

Figure 4.19: Graph showing longitudinal shear modulus (G12) plotted against the nanotube lengths $(\mathrm{nm})$ for varying volume fractions of nanotubes aligned uniaxially with the LCP molecules. 


\section{Summary and Conclusions}

\subsection{Summary}

The research was conducted to study the effects of using a liquid crystalline polymer as a matrix material in a composite in order to customize the alignment of the nanotubes as per the requirement of the structural applications. The motif was to use the property of LCP monomers of being able to be oriented in the preferred direction with the help of electric or magnetic fields and to lock in the arrangement when the field was removed. It was necessary to develop a mathematical model that would accurately predict the elastic properties of the final composite. A mathematical model was created with the help of MATLAB, encompassing the numerical scheme developed (Gavazzi and Lagoudas 1990) to evaluate the components of the Eshelby's tensor $\mathrm{S}_{\mathrm{ijkl}}$. This scheme was then used to evaluate the properties of the final composite using the Mori Tanaka method (G. M. Odegard, et al. 2003) taking into consideration the effects of nanotube concentration and orientation on the final properties of the composite. It was predicted that even small concentrations of nanotubes when combined with LCPs could produce outstanding results that could be taken advantage of for numerous applications. Results for the longitudinal Young's modulus (E1), transverse Young's modulus (E2) and the longitudinal shear modulus (G12) were generated for nanotube volume fractions ranging from 0 to 0.25 and orientations ranging from 0 to 90 degrees in relation to the LCP monomers. An overview of the data obtained has been given in the results section of this document. Exceptionally high modulus values were obtained for nanotubes aligned in the longitudinal direction of the composite material providing greater stiffness in the longitudinal direction whereas the transverse side of the composite was found to be weaker to sustain the order of loading that the longitudinal side was capable of. When the nanotubes were rotated by 90 degrees or when they were perpendicular to the axis of the LCP monomers, the stiffness in the transverse direction was found to be much 
greater than that in the longitudinal direction. It was also found that the length only had an effect on the properties until the nanotubes of $250 \mathrm{~nm}$ were used. Nanotubes above the length of $250 \mathrm{~nm}$ hardly affected the properties of the composite, which is clearly visible from the graph in Figure 4.1.

\subsection{Conclusions}

A mathematical model has been successfully developed to calculate the components of the Eshelby's tensor and ultimately to calculate the mechanical properties for a general composite material. This model has been used to calculate the mechanical properties of the $\mathrm{LCP} /$ nanotube composite for various cases of nanotube shape, size, orientation and volume fraction. Thus, the model helped in studying the constitutive behavior of a composite based on numerous parameters.

The data obtained from the research study was plotted in various graphs and can be seen in the results section of this document. It is of importance to note that nanotubes, when combined with LCPs, generate remarkable properties. These properties, as stated earlier, depend upon the length and the orientation of the nanotubes. It was observed that this enhancement in properties of the composite is observed only until the nanotubes of a certain aspect ratio are used. Once, the aspect ratio increases and becomes more than this critical value, the properties of the composite are not improved further. This is because of the tendency of the nanotubes to be more flexible at higher aspect ratios resulting in the deterioration of the efficiency of load transfer. This critical value for the nanotubes has been found to be the length of about $250 \mathrm{~nm}$. It can be observed from the data plotted in the graphs that for longer nanotubes (up to $250 \mathrm{~nm}$ ), a linear relationship exists between the longitudinal modulus and the nanotube volume fraction. For short fiber composites with aligned nanotubes, stiffness values are very low at low concentration of nanotubes. Again, for nanotubes oriented at different angles with respect to the longitudinal direction of the 
composite, the theory is no different, but the longitudinal modulus goes on decreasing with the increase in orientation. This is because of the fact that the longitudinal modulus of the LCP is much less than that of the nanotubes and thus, the longitudinal modulus of the composite is governed by the properties of the nanotubes.

It is conclusive of the results that the properties of the final composite formed out of $\mathrm{LCP} /$ nanotube are nanotube dominated and change with every single variation in the nanotube shape, size, orientation and volume fraction. It has been proven that even the minutest concentrations of the nanotubes, equivalent to about $5 \%$ by volume, generate significant results for the LCP/nanotube composite. As a result the composites formed are extremely lightweight and can be used in various applications. Also, the material is stiffer than most composite materials thus contributing to its high stiffness to weight ratio. 


\section{Bibliography}

Baughman, R. H., A. A. Zakhidov and W. A. de Heer. "Carbon Nanotubes- the route towards applications." Science, 297(5582), 2002: 787-792.

Benveniste, Y. "A new approach to the application of Mori-Tanaka's theory in composite materials." Mechanics of Materials, 6, 1987: 147-157.

Daniel, I. M., O. Ishai. "Engineering Mechanics of Composite Materials.” New York: Oxford University Press, 3, 1994.

Eshelby, J. D. "The determination of the elastic field of an ellipsoidal inclusion, and related problems." The Royal Society, 1957: 376-396.

Fukudu H., Y. Takao. "Thermoelastic properties of discontinuous fiber composites." Comprehensive Composite Materials, 1, 2000: 377-401.

Gavazzi, A. C., D. C. Lagoudas, "On the numerical evaluation of Eshelby's tensor and its application to elastoplastic fibrous composites." Computational Mechanics, 7 , 1990: 13-19.

Kimura, T., H. Ago, M. Tobita, S. Ohshima, M. Kyotani and M. Yumura. "Polymer composites of carbon nanotubes aligned by a magnetic field." Advanced Materials, 14, 2002: 1380-1383.

Mori, T. and K. Tanaka. "Average stress in matrix and average elastic energy of materials with misfitting inclusions.” Acta Metallurgica, 21(5), 1973:571-574.

Odegard, G. M., T. S. Gates, K. E. Wise, C. Park and E. J. Siochi. "Constitutive modeling of nanotube-reinforced polymer composites." Composites Science and Technology, 63, 2003: 1671-1683.

Odegard, G. M., S. J. V. Frankland and T. S. Gates. "Effect of nanotube functionalization on the elastic properties of polyethylene nanotube composites." The American Institute of Aeronautics and Astronautics, 43(8), 2005: 1828-1835. 
Qiu, Y. P. and G. J. Weng. "On the application of Mori-Tanaka's theory involving transversely isotropic spheroidal inclusions." International Journal of Engineering Science, 28(11), 1990: 1121-1137.

Wang, X., J. Engle and C. Liu. "Liquid crystal polymer for MEMS: processes and applications." Journal of Micromechanics and Microengineering, 13, 2003: 628-633. Yu, M. F., O. Lourie, M. J. Dyer, K. Moloni, T. F. Kelly and R. S. Rouff. "Strength and breaking mechanism of multiwalled carbon nanotubes under tensile load." Science, 287, 2000: 637-640. 


\section{Appendices}

\subsection{Appendix A - Material Properties}

The materials used in this research, the phases of a composite material, were the liquid crystalline polymer and the carbon nanotubes. Although, they have been referred to as the nanotubes in the entire document, their properties represent the behavior of an effective fiber. An effective fiber takes into account the interaction properties between the two phases and has been discussed earlier in this document. The properties of the polymer and the effective fiber are given in table 7.1.
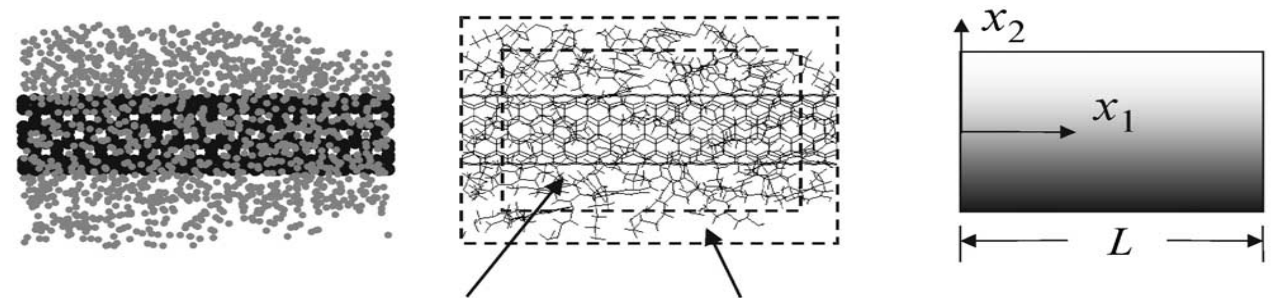

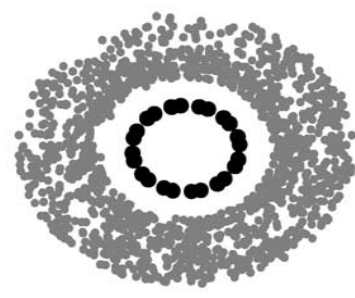

Molecular model

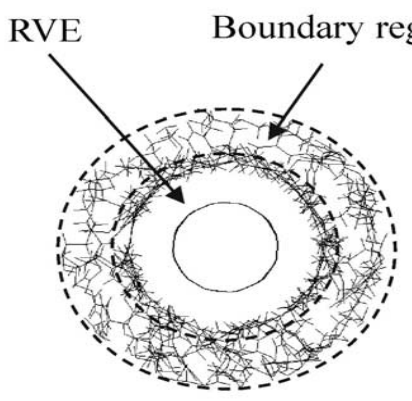

Equivalent-truss model

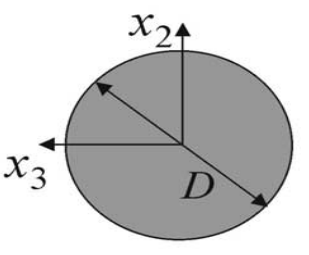

Equivalentcontinuum model

Figure 7.1: Equivalent continuum modeling of effective fiber (figure 7.1 is copyrighted and used with permission of Composites Science and Technology, all rights reserved, see Appendix-C) 
Table 7.1: Elastic properties of Liquid Crystalline Polymer

\begin{tabular}{|c|c|}
\hline Property & Magnitude \\
\hline Longitudinal Young's modulus, E11a & $2 \mathrm{GPa}$ \\
\hline Transverse Young's modulus, E22a & $1.5 \mathrm{GPa}$ \\
\hline Longitudinal Poisson's ratio, v12a & 0.3 \\
\hline Transverse Poisson's ratio, v23a & 0.25 \\
\hline Longitudinal shear modulus, G12a & $1.4 \mathrm{GPa}$ \\
\hline
\end{tabular}

These properties have been obtained from the experimental data provided by Chartoff, et al. and can be easily drawn into the stiffness matrix by using general rules of the mechanics of composite materials. The properties for the effective fiber have been directly extracted from the work of Odegard et al., (G.M. Odegard, et al. 2003). They directly represent the components of the stiffness tensor for the effective fiber and are as given below in table 7.2. Odegard, et al. used molecular dynamics and equivalent continuum modeling techniques in order to compute the properties for the effective fiber.

Table 7.2: Components of the stiffness tensor for effective fiber (G. M. Odegard, et al. 2003).

\begin{tabular}{|c|c|}
\hline Component & Magnitude (GPa) \\
\hline $\mathrm{C}_{11}$ & 457.6 \\
\hline $\mathrm{C}_{12}$ & 8.4 \\
\hline $\mathrm{C}_{22}$ & 14.3 \\
\hline $\mathrm{C}_{23}$ & 5.5 \\
\hline $\mathrm{C}_{44}$ & 27.0 \\
\hline
\end{tabular}


Table 7.3: Stiffness matrix for the composite obtained using 500nm long nanotubes aligned axisymmetrically with the polymer at a $25 \%$ volume concentration.

$10^{11} \mathrm{x}$
\begin{tabular}{|c|c|c|c|c|c|c|c|c|}
\hline 3.3712 & 0.0637 & 0.0637 & 0 & 0 & 0 & 0 & 0 & 0 \\
\hline 0.0637 & 0.1100 & 0.0421 & 0 & 0 & 0 & 0 & 0 & 0 \\
\hline 0.0637 & 0.1100 & 0.0421 & 0 & 0 & 0 & 0 & 0 & 0 \\
\hline 0 & 0 & 0 & 0.0339 & 0 & 0 & 0.0339 & 0 & 0 \\
\hline 0 & 0 & 0 & 0 & 0.1203 & 0 & 0 & 0.1203 & 0 \\
\hline 0 & 0 & 0 & 0 & 0 & 0.1203 & 0 & 0 & 0.1203 \\
\hline 0 & 0 & 0 & 0.0339 & 0 & 0 & 0.0339 & 0 & 0 \\
\hline 0 & 0 & 0 & 0 & 0.1203 & 0 & 0 & 0.1203 & 0 \\
\hline 0 & 0 & 0 & 0 & 0 & 0.1203 & 0 & 0 & 0.1203 \\
\hline
\end{tabular}

The inverse of the above stiffness matrix gives the compliance matrix for the composite using which the elastic constants for the composite can be easily calculated using simple arithmetic operations as shown below. Let the compliance matrix obtained from the above stiffness matrix be denoted by $S^{\prime}$. Then, for the composite material,

$$
\begin{aligned}
& \mathrm{E} 1=1 / \mathrm{S}^{\prime}(1,1) \\
& \mathrm{E} 2=1 / \mathrm{S}^{\prime}(2,2) \\
& \mathrm{v} 12=-\mathrm{S}^{\prime}(1,2)^{*} \mathrm{E} 1 \\
& \mathrm{v} 23=-\mathrm{S}^{\prime}(2,3)^{*} \mathrm{E} 2 \\
& \mathrm{G} 12=1 / \mathrm{S}^{\prime}(6,6)
\end{aligned}
$$




\subsection{Appendix B- MATLAB Model and Explanation}

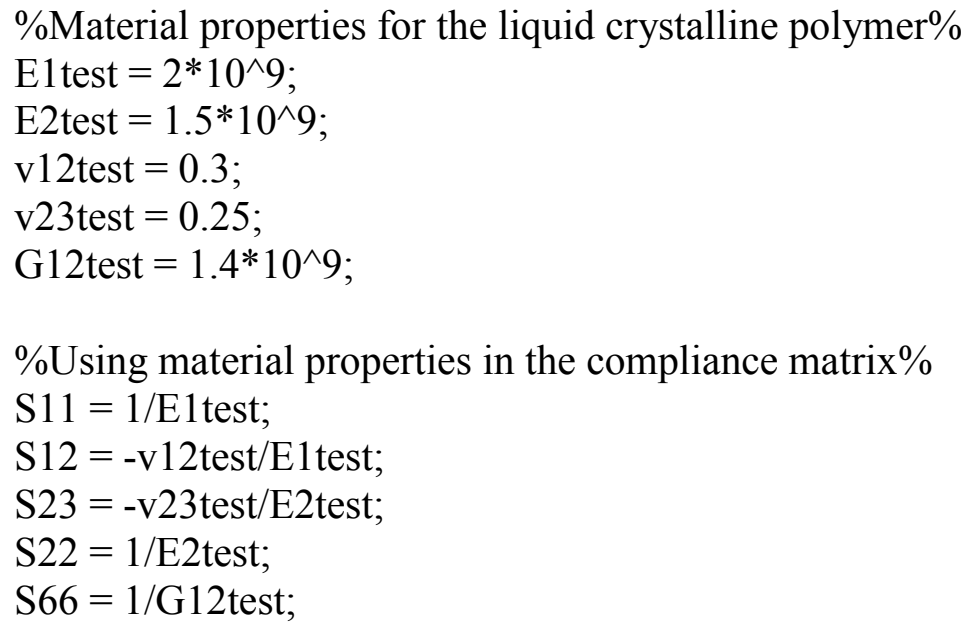

$\%$ Using material properties in the compliance matrix $\%$

$\mathrm{S} 11=1 /$ E1test;

$\mathrm{S} 12=-\mathrm{v} 12$ test $/$ E1 test;

$\mathrm{S} 23=-\mathrm{v} 23$ test/E2test;

$\mathrm{S} 22=1 /$ E2test;

$\mathrm{S} 66=1 / \mathrm{G} 12$ test;

\%Compliance matrix, conversion to stiffness matrix \%

$\mathrm{S} 1=[\mathrm{S} 11$ S12 S12 00 0;S12 S22 S23 00 0;S12 S23 S22 000 0;0 00 (2*(S22-S23)) 0 0;0 $000 \mathrm{~S} 66$ 0;0 0000 S66];

$\mathrm{C} 1=\operatorname{inv}(\mathrm{S} 1)$

$\%$ Stiffness matrix- A matrix of order $9 \%$

$\mathrm{L}=[\mathrm{C} 1(1,1) \mathrm{C} 1(1,2) \mathrm{C} 1(1,3) \mathrm{C} 1(1,4) \mathrm{C} 1(1,5) \mathrm{C} 1(1,6) 00$ 0; $1(2,1) \mathrm{C} 1(2,2) \mathrm{C} 1(2,3)$ $\mathrm{C} 1(2,4) \mathrm{C} 1(2,5) \mathrm{C} 1(2,6) 00$ 0; $1(3,1) \mathrm{C} 1(3,2) \mathrm{C} 1(3,3) \mathrm{C} 1(3,4) \mathrm{C} 1(3,5) \mathrm{C} 1(3,6) 00$ 0;0 $00 \mathrm{C} 1(4,4) 00 \mathrm{C} 1(4,4) 00 ; 0000 \mathrm{C} 1(5,5) 00 \mathrm{C} 1(5,5) \quad 0 ; 000000 \mathrm{C} 1(6,6) 00$ $\mathrm{C} 1(6,6) ; 000 \mathrm{C} 1(4,4) 00 \mathrm{C} 1(4,4) 00$ 0;0 $0000 \mathrm{C} 1(5,5) 00 \mathrm{C} 1(5,5)$ 0;0 $00000 \mathrm{C} 1(6,6)$ $00 \mathrm{C} 1(6,6)]$;

$\%$ Stiffness matrix for the effective fiber $\%$

$\mathrm{Cf1}=\left(10^{\wedge} 9\right)^{*}[457.68 .48 .40000000 ; 8.414 .35 .500000000 ; 8.45 .514 .3000000$ 0;0 $004.4004 .400 ; 0000270027$ 0;0 $000002700027 ; 0004.4004 .400$ 0;0 0 $002700270 ; 000000270027$;;

$\mathrm{I}=\operatorname{eye}(9) ; \%$ Identity matrix $\%$ $\mathrm{c} 123=1 ; \mathrm{c} 231=1 ; \mathrm{c} 132=-1 ; \mathrm{c} 213=-1 ; \mathrm{c} 312=1 ; \mathrm{c} 321=-1$; permutation tensors a1 $=1.8^{*} 10^{\wedge}-9 ;$ a $2=1.8^{*} 10^{\wedge}-9 ;$ a3 $=500^{*} 10^{\wedge}-9$; nanotube properties $\mathrm{c}=1 ; \mathrm{d}=2$;

$\%$ Gaussian Weights for 96 iterations of the Numerical Integration\%

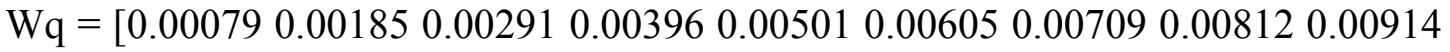
$\begin{array}{lllllllll}0.01016 & 0.01116 & 0.01215 & 0.01312 & 0.01409 & 0.01503 & 0.01597 & 0.01688 & 0.01778\end{array}$ $\begin{array}{llllllllll}0.01866 & 0.01951 & 0.02035 & 0.02117 & 0.02196 & 0.02273 & 0.02348 & 0.02420 & 0.02490\end{array}$ $\begin{array}{llllllllll}0.02557 & 0.02621 & 0.02682 & 0.02741 & 0.02797 & 0.02849 & 0.02899 & 0.02946 & 0.02989\end{array}$ $\begin{array}{llllllllll}0.03029 & 0.03067 & 0.03101 & 0.03131 & 0.03158 & 0.03182 & 0.03203 & 0.03220 & 0.03234\end{array}$ $\begin{array}{llllllllll}0.03244 & 0.03251 & 0.03255 & 0.03255 & 0.03251 & 0.03244 & 0.03234 & 0.03220 & 0.03203\end{array}$ $\begin{array}{llllllllll}0.03182 & 0.03158 & 0.03131 & 0.03101 & 0.03067 & 0.03029 & 0.02989 & 0.02946 & 0.02899\end{array}$ 
$\begin{array}{lllllllll}0.02849 & 0.02797 & 0.02741 & 0.02682 & 0.02621 & 0.02557 & 0.02490 & 0.02420 & 0.02348 \\ 0.02273 & 0.02196 & 0.02117 & 0.02035 & 0.01951 & 0.01866 & 0.01778 & 0.01688 & 0.01597 \\ 0.01503 & 0.01409 & 0.01312 & 0.01215 & 0.01116 & 0.01016 & 0.00914 & 0.00812 & 0.00709 \\ 0.00605 & 0.00501 & 0.00396 & 0.00291 & 0.00185 & 0.00079] ;\end{array}$

$\%$ This part calculates the values of all components of $\mathrm{G}_{\mathrm{ijkl}}(\bar{\xi})$ for positive and negative values of eta $3\left(\zeta_{3}\right) \%$

omga $=(2 *$ pi/96);

for $\mathrm{i}=1: 96$;

for $\mathrm{p}=1$

if $\mathrm{p}=\mathrm{c}$

eta3 $=0.577350269189626$;

elseif $\mathrm{p}==\mathrm{d}$

eta $3=-0.577350269189626$;

end

eta $1=\left(\operatorname{sqrt}\left(1-\left((\text { eta3 })^{\wedge} 2\right)\right) *(\cos (\right.$ omga $\left.* i))\right)$;

eta2 $=\left(\operatorname{sqrt}\left(1-\left((\text { eta } 3)^{\wedge} 2\right)\right) *(\sin (\right.$ omga*i) $))$;

zeta1 $=$ eta $1 / \mathrm{a} 1 ; \%$ zeta1 $=0 ; \%$ zeta $(\xi) \%$

zeta $2=$ eta $2 / \mathrm{a} 2 ; \%$ zeta $2=0 ; \%$ use either of the values depending on $\mathrm{a}_{1}, \mathrm{a}_{2}, \mathrm{a}_{3}$

$\%$ zeta $3=$ eta $3 / \mathrm{a} 3 ;$ zeta $3=0$;

$\mathrm{K} 11=\mathrm{L}(1,1) *$ zeta 1 *zeta $1+\mathrm{L}(6,6) *$ zeta2*zeta $2+\mathrm{L}(8,8) *$ zeta3*zeta3 + $\mathrm{L}(1,6) *$ zeta 1 *zeta2 $+\mathrm{L}(1,8) *$ zeta 1 *zeta3 $+\mathrm{L}(6,1) *$ zeta $2 *$ zeta $1+\mathrm{L}(6,8) *$ zeta $2 *$ zeta3 $+\mathrm{L}(8,6) *$ zeta $3 *$ zeta $2+\mathrm{L}(8,1) *$ zeta $3 *$ zeta 1

$\mathrm{K} 12=\mathrm{L}(1,9) *$ zeta1*zeta $1+\mathrm{L}(6,2) *$ zeta2*zeta $2+\mathrm{L}(8,4) *$ zeta3*zeta3 + $\mathrm{L}(1,2) *$ zeta 1 *zeta 2 L $(1,4) *$ zeta 1 *zeta $3+\mathrm{L}(6,9) *$ zeta2*zeta1 + L(6,4)*zeta2*zeta3 $+\mathrm{L}(8,2) *$ zeta3*zeta2 $+\mathrm{L}(8,9) *$ zeta3*zeta $;$

$\mathrm{K} 13=\mathrm{L}(1,5) *$ zeta 1 zeta $1+\mathrm{L}(6,7) *$ zeta2*zeta $2+\mathrm{L}(8,3) *$ zeta3*zeta3 + $\mathrm{L}(1,7) *$ zeta1 *zeta2 + L(1,3)*zeta 1 *zeta3 $+\mathrm{L}(6,5) *$ zeta2*zeta1 + L(6,3)*zeta2*zeta3 $+\mathrm{L}(8,7) *$ zeta3*zeta2 $+\mathrm{L}(8,5) *$ zeta3*zeta $;$

$\mathrm{K} 21=\mathrm{L}(9,1) *$ zeta1*zeta $1+\mathrm{L}(2,6) *$ zeta2*zeta $2+\mathrm{L}(4,8) *$ zeta3*zeta3 + $\mathrm{L}(9,6) *$ zeta 1 *zeta2 $+\mathrm{L}(9,8) *$ zeta 1 *zeta3 $+\mathrm{L}(2,1) *$ zeta2*zeta $1+\mathrm{L}(2,8) *$ zeta $2 *$ zeta3 $+\mathrm{L}(4,6) *$ zeta $3 *$ zeta $2+\mathrm{L}(4,1) *$ zeta3*zeta 1

$\mathrm{K} 22=\mathrm{L}(9,9) *$ zeta 1 zeta $1+\mathrm{L}(2,2) *$ zeta2*zeta $2+\mathrm{L}(4,4) *$ zeta3*zeta3 + $\mathrm{L}(9,2) * z e t a 1 * z e t a 2+\mathrm{L}(9,4) *$ zeta 1 *zeta3 $+\mathrm{L}(2,9) *$ zeta2*zeta $1+\mathrm{L}(2,4) *$ zeta2*zeta3 $+\mathrm{L}(4,2) *$ zeta3*zeta2 + L $(4,9) *$ zeta3*zeta $;$

$\mathrm{K} 23=\mathrm{L}(9,5) *$ zeta 1 zeta $1+\mathrm{L}(2,7){ }^{*}$ zeta2*zeta $2+\mathrm{L}(4,3) *$ zeta3*zeta3 + $\mathrm{L}(9,7) *$ zeta1*zeta2 + L $(9,3) *$ zeta 1 *zeta $3+\mathrm{L}(2,5) *$ zeta2*zeta1 + L $(2,3) *$ zeta2*zeta3 $+\mathrm{L}(4,7) *$ zeta3*zeta2 + L $(4,5) *$ zeta3*zeta1;

$\mathrm{K} 31=\mathrm{L}(5,1) *$ zeta 1 zeta $1+\mathrm{L}(7,6))^{*}$ zeta2*zeta $2+\mathrm{L}(3,8) *$ zeta3*zeta3 + $\mathrm{L}(5,6) *$ zeta1 *zeta2 + L $(5,8) *$ zeta $1 *$ zeta3 $+\mathrm{L}(7,1) *$ zeta2*zeta $1+\mathrm{L}(7,8) *$ zeta2*zeta3 $+\mathrm{L}(3,6) *$ zeta3*zeta2 + L $(3,1) *$ zeta3*zeta1; 
$\mathrm{K} 32=\mathrm{L}(5,9) *$ zeta 1 zeta $1+\mathrm{L}(7,2) *$ zeta2*zeta $2+\mathrm{L}(3,4) *$ zeta3*zeta3 + $\mathrm{L}(5,2) * z e t a 1 * z e t a 2+\mathrm{L}(5,4) *$ zeta 1 *zeta3 $+\mathrm{L}(7,9) *$ zeta2*zeta1 $+\mathrm{L}(7,4) *$ zeta2*zeta3 $+\mathrm{L}(3,2) *$ zeta3*zeta2 + L(3,9)*zeta3*zeta1;

$\mathrm{K} 33=\mathrm{L}(5,5) *$ zeta $1 *$ zeta $1+\mathrm{L}(7,7) *$ zeta $2 *$ zeta $2+\mathrm{L}(3,3) *$ zeta3*zeta3 + $\mathrm{L}(5,7) *$ zeta 1 *zeta2 $+\mathrm{L}(5,3) *$ zeta 1 *zeta $3+\mathrm{L}(7,5) *$ zeta2*zeta $1+\mathrm{L}(7,3) *$ zeta2*zeta3 $+\mathrm{L}(3,7) *$ zeta3*zeta2 $+\mathrm{L}(3,5) *$ zeta3*zeta1;

$\mathrm{D}=\mathrm{c} 123 * \mathrm{~K} 11 * \mathrm{~K} 22 * \mathrm{~K} 33+\mathrm{c} 231 * \mathrm{~K} 21 * \mathrm{~K} 32 * \mathrm{~K} 13+\mathrm{c} 312 * \mathrm{~K} 31 * \mathrm{~K} 12 * \mathrm{~K} 23+$ $\mathrm{c} 132 * \mathrm{~K} 11 * \mathrm{~K} 32 * \mathrm{~K} 23+\mathrm{c} 213 * \mathrm{~K} 21 * \mathrm{~K} 12 * \mathrm{~K} 33+\mathrm{c} 321 * \mathrm{~K} 31 * \mathrm{~K} 22 * \mathrm{~K} 13$;

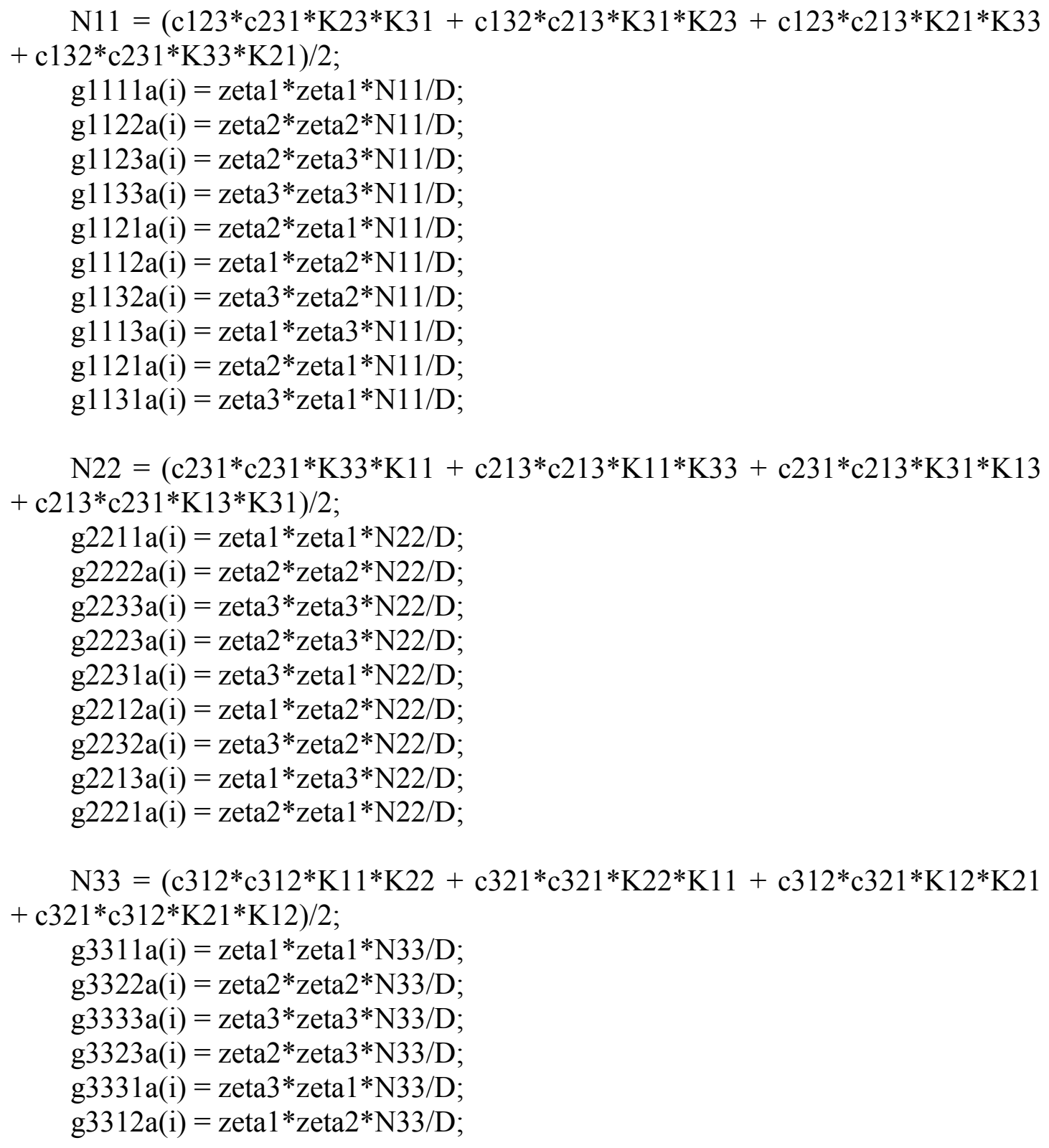




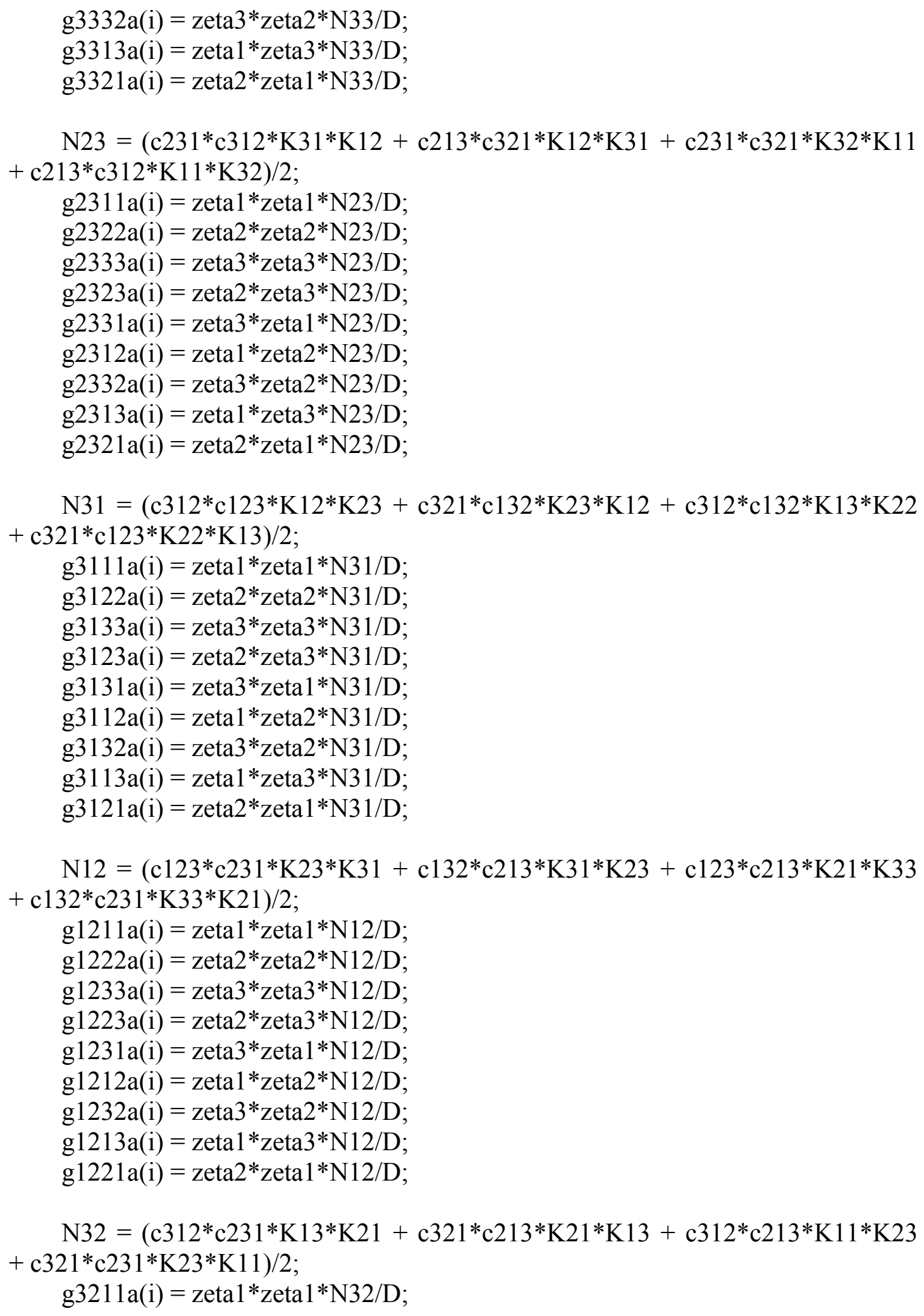




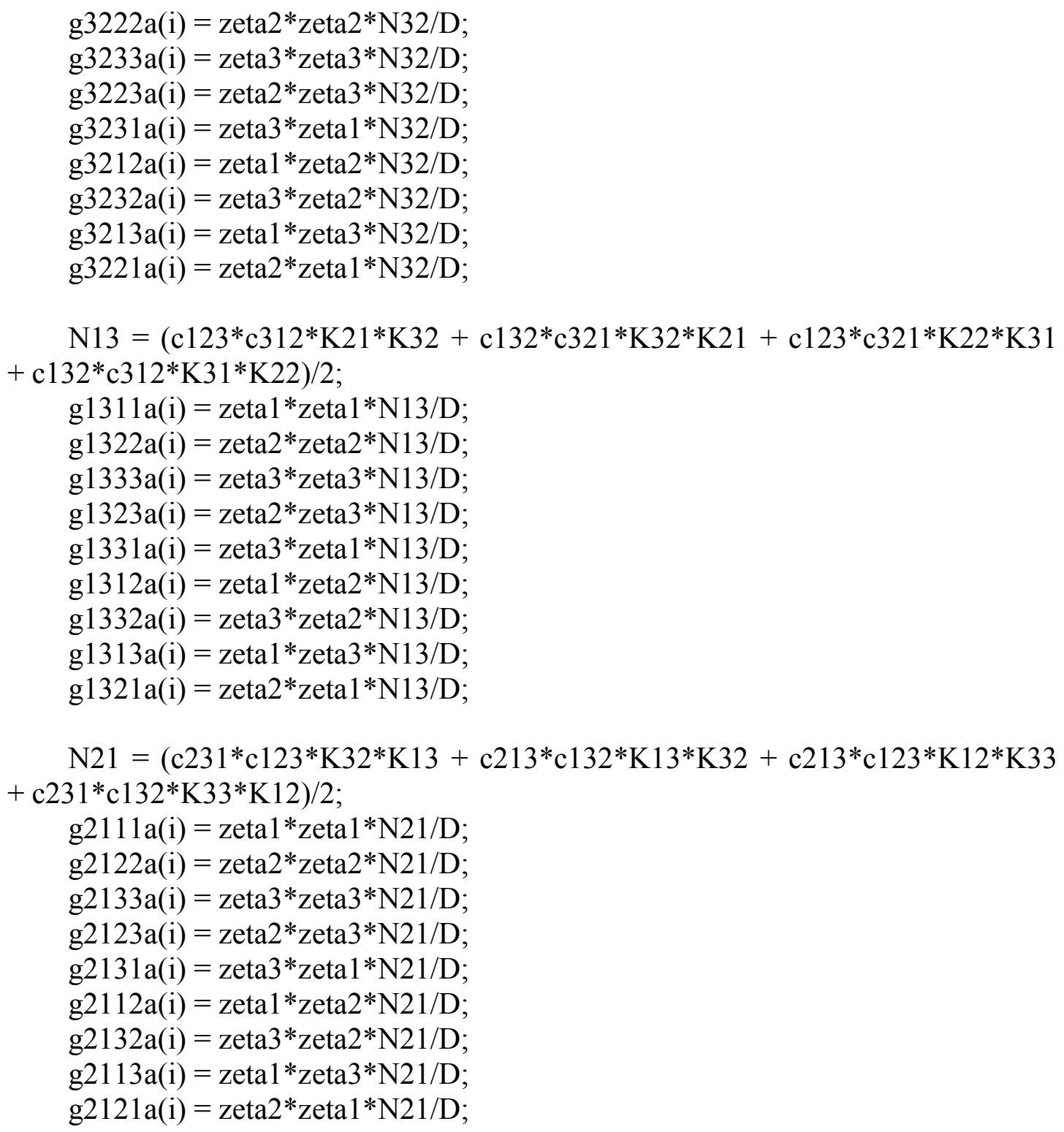

end

for $\mathrm{p}=2$;

$$
\begin{aligned}
& \text { if } \mathrm{p}==\mathrm{c} \\
& \quad \text { eta } 3=0.577350269189626 \\
& \text { elseif } \mathrm{p}==\mathrm{d} \\
& \quad \text { eta } 3=-0.577350269189626 \\
& \text { end }
\end{aligned}
$$


eta2 $=\left(\operatorname{sqrt}\left(1-\left((\text { eta } 3)^{\wedge} 2\right)\right) *(\sin (\right.$ omga $\left.* \mathrm{i}))\right)$

zeta $1=$ eta $1 / \mathrm{a} 1 ; \%$ zeta $1=0$

zeta $2=$ eta $2 /$ a $2 ; \%$ zeta2 $=0$;

$\%$ zeta $3=$ eta $3 / \mathrm{a} 3 ;$ zeta $3=0$;

$\mathrm{K} 11=\mathrm{L}(1,1) *$ zeta 1 zeta $1+\mathrm{L}(6,6) *$ zeta2*zeta $2+\mathrm{L}(8,8) *$ zeta3*zeta3 + $\mathrm{L}(1,6)$ *zeta 1 *zeta2 + L(1,8)*zeta1*zeta3 + L(6,1)*zeta2*zeta1 + L(6,8)*zeta2*zeta3 $+\mathrm{L}(8,6) *$ zeta3*zeta $2+\mathrm{L}(8,1) *$ zeta3*zeta1;

$\mathrm{K} 12=\mathrm{L}(1,9) *$ zeta 1 zeta $1+\mathrm{L}(6,2) *$ zeta2*zeta $2+\mathrm{L}(8,4) *$ zeta3*zeta3 + $\mathrm{L}(1,2) *$ zeta 1 *zeta2 + L $(1,4) *$ zeta 1 *zeta3 $+\mathrm{L}(6,9) *$ zeta2*zeta1 + L(6,4)*zeta2*zeta3 $+\mathrm{L}(8,2) *$ zeta3*zeta2 $+\mathrm{L}(8,9) *$ zeta3*zeta $;$

$\mathrm{K} 13=\mathrm{L}(1,5) *$ zeta 1 *zeta $1+\mathrm{L}(6,7) *$ zeta2*zeta $2+\mathrm{L}(8,3) *$ zeta3*zeta3 + $\mathrm{L}(1,7) *$ zeta $1 *$ zeta2 $+\mathrm{L}(1,3) *$ zeta 1 *zeta $3+\mathrm{L}(6,5) *$ zeta $2 *$ zeta $1+\mathrm{L}(6,3) *$ zeta $2 * z e t a 3$ $+\mathrm{L}(8,7) *$ zeta3*zeta2 $+\mathrm{L}(8,5) *$ zeta3*zeta $;$

$\mathrm{K} 21=\mathrm{L}(9,1) *$ zeta 1 *zeta $1+\mathrm{L}(2,6) *$ zeta2*zeta $2+\mathrm{L}(4,8) *$ zeta3*zeta3 + $\mathrm{L}(9,6) *$ zeta 1 *zeta2 $+\mathrm{L}(9,8) *$ zeta 1 *zeta3 $+\mathrm{L}(2,1) *$ zeta2*zeta1 + L $(2,8) *$ zeta2*zeta3 $+\mathrm{L}(4,6) *$ zeta3*zeta2 $+\mathrm{L}(4,1) *$ zeta3*zeta1;

$\mathrm{K} 22=\mathrm{L}(9,9) *$ zeta1*zeta $+\mathrm{L}(2,2) *$ zeta2*zeta $2+\mathrm{L}(4,4) *$ zeta3*zeta3 + $\mathrm{L}(9,2) * z e t a 1$ *zeta2 $+\mathrm{L}(9,4) *$ zeta 1 *zeta3 $+\mathrm{L}(2,9) *$ zeta 2 *zeta $1+\mathrm{L}(2,4) *$ zeta2*zeta3 $+\mathrm{L}(4,2) * z e t a 3 * z e t a 2+\mathrm{L}(4,9) *$ zeta3*zeta $;$

$\mathrm{K} 23=\mathrm{L}(9,5) *$ zeta 1 zzeta $1+\mathrm{L}(2,7) *$ zeta2*zeta $2+\mathrm{L}(4,3) *$ zeta3*zeta3 + $\mathrm{L}(9,7) *$ zeta $1 *$ zeta2 $+\mathrm{L}(9,3) *$ zeta 1 *zeta3 $+\mathrm{L}(2,5) *$ zeta $2 *$ zeta $1+\mathrm{L}(2,3) *$ zeta2*zeta3 $+\mathrm{L}(4,7) *$ zeta3*zeta2 $+\mathrm{L}(4,5) *$ zeta3*zeta $;$

$\mathrm{K} 31=\mathrm{L}(5,1) *$ zeta 1 *zeta $1+\mathrm{L}(7,6) *$ zeta2*zeta $2+\mathrm{L}(3,8) *$ zeta3*zeta3 + $\mathrm{L}(5,6) *$ zeta1 *zeta2 + L $(5,8) *$ zeta 1 *zeta3 + L(7,1)*zeta2*zeta $+\mathrm{L}(7,8) * z e t a 2 * z e t a 3$ $+\mathrm{L}(3,6) *$ zeta3*zeta $2+\mathrm{L}(3,1) *$ zeta3*zeta 1 ;

$\mathrm{K} 32=\mathrm{L}(5,9) *$ zeta $1 *$ zeta $1+\mathrm{L}(7,2) *$ zeta2*zeta $2+\mathrm{L}(3,4) *$ zeta3*zeta3 + $\mathrm{L}(5,2) *$ zeta $1 *$ zeta2 $+\mathrm{L}(5,4) *$ zeta 1 *zeta $3+\mathrm{L}(7,9) *$ zeta2*zeta1 $+\mathrm{L}(7,4) *$ zeta2*zeta3 $+\mathrm{L}(3,2) *$ zeta3*zeta2 $+\mathrm{L}(3,9) *$ zeta3*zeta $;$

$\mathrm{K} 33=\mathrm{L}(5,5) *$ zeta 1 zeta $1+\mathrm{L}(7,7) *$ zeta2*zeta $2+\mathrm{L}(3,3) *$ zeta3*zeta3 + $\mathrm{L}(5,7) *$ zeta $1 *$ zeta2 $+\mathrm{L}(5,3) *$ zeta $1 *$ zeta3 $+\mathrm{L}(7,5) *$ zeta $2 *$ zeta $1+\mathrm{L}(7,3) *$ zeta2*zeta3 $+\mathrm{L}(3,7) *$ zeta3*zeta2 + L $(3,5) *$ zeta3*zeta1;

$\mathrm{D}=\mathrm{c} 123 * \mathrm{~K} 11 * \mathrm{~K} 22 * \mathrm{~K} 33+\mathrm{c} 231 * \mathrm{~K} 21 * \mathrm{~K} 32 * \mathrm{~K} 13+\mathrm{c} 312 * \mathrm{~K} 31 * \mathrm{~K} 12 * \mathrm{~K} 23+$ $\mathrm{c} 132 * \mathrm{~K} 11 * \mathrm{~K} 32 * \mathrm{~K} 23+\mathrm{c} 213 * \mathrm{~K} 21 * \mathrm{~K} 12 * \mathrm{~K} 33+\mathrm{c} 321 * \mathrm{~K} 31 * \mathrm{~K} 22 * \mathrm{~K} 13$;

$\mathrm{N} 11=(\mathrm{c} 123 * \mathrm{c} 231 * \mathrm{~K} 23 * \mathrm{~K} 31+\mathrm{c} 132 * \mathrm{c} 213 * \mathrm{~K} 31 * \mathrm{~K} 23+\mathrm{c} 123 * \mathrm{c} 213 * \mathrm{~K} 21 * \mathrm{~K} 33$ $+\mathrm{c} 132 * \mathrm{c} 231 * \mathrm{~K} 33 * \mathrm{~K} 21) / 2$

$\mathrm{g} 1111 \mathrm{~b}(\mathrm{i})=$ zeta $1 *$ zeta $1 * \mathrm{~N} 11 / \mathrm{D}$;

g1122b(i) $=$ zeta $2 *$ zeta $2 * \mathrm{~N} 11 / \mathrm{D}$;

g1123b(i) $=$ zeta $2 *$ zeta $3 *$ N11/D;

g1133b(i) = zeta3*zeta $3 *$ N11/D; 


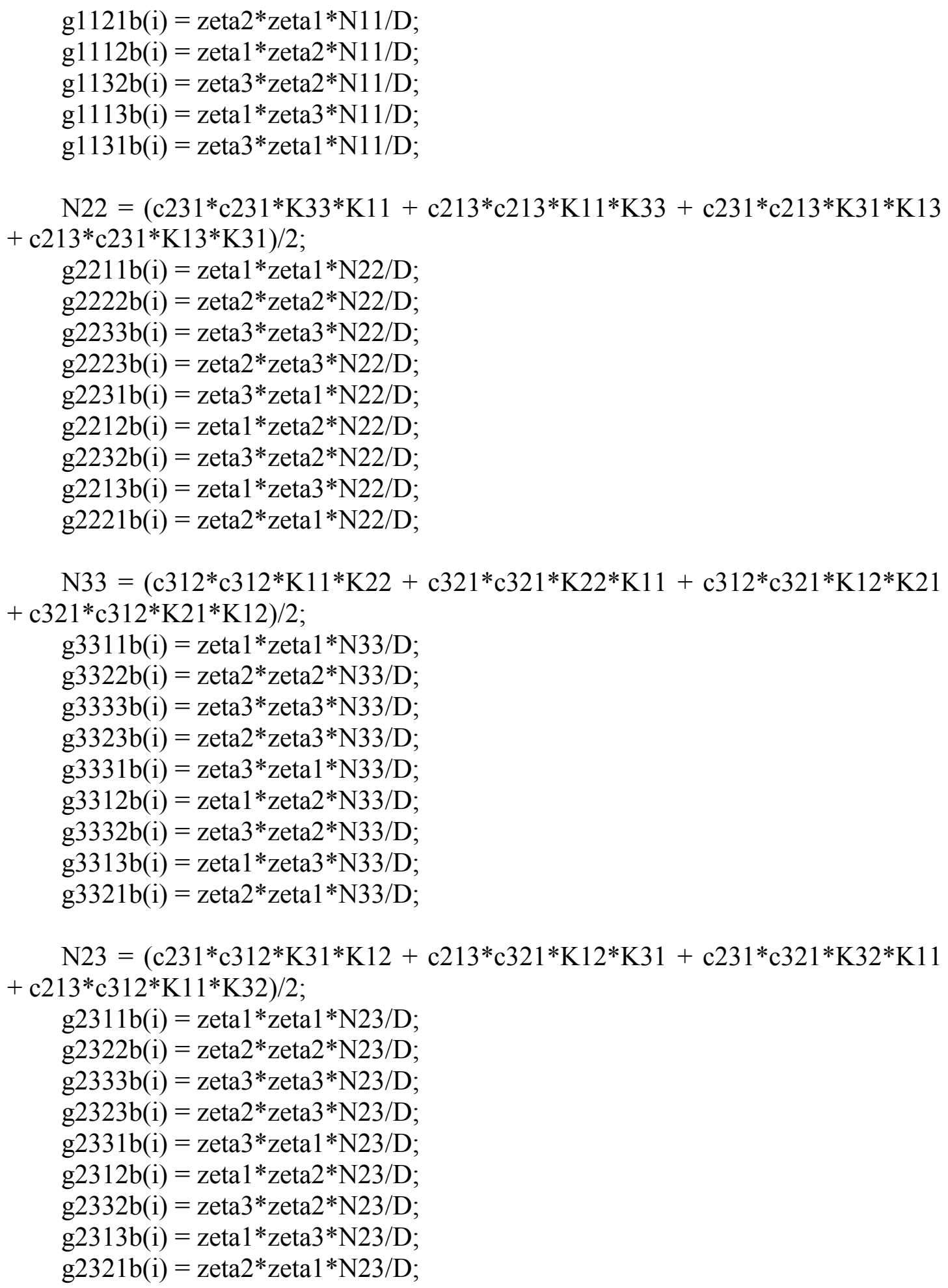




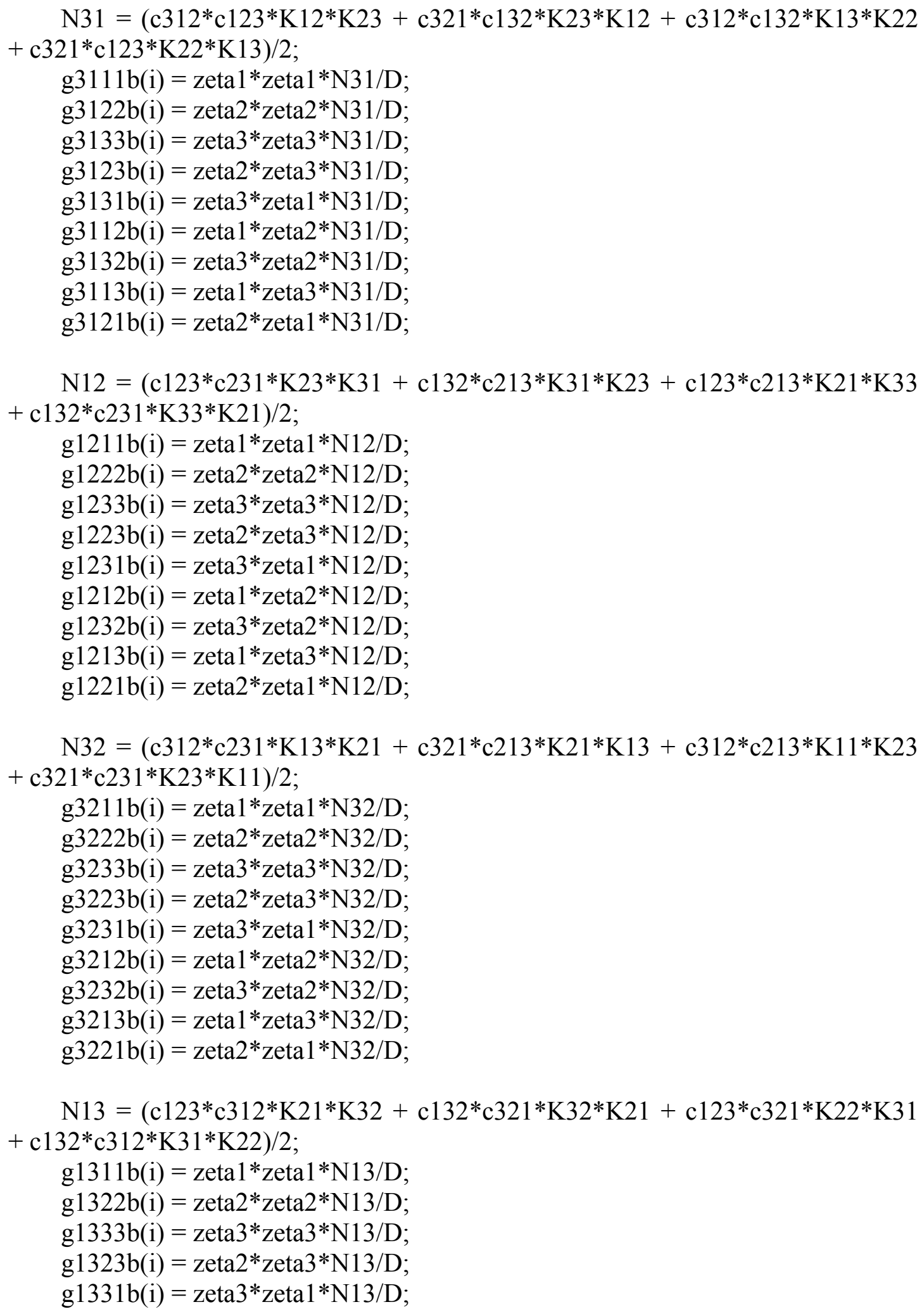




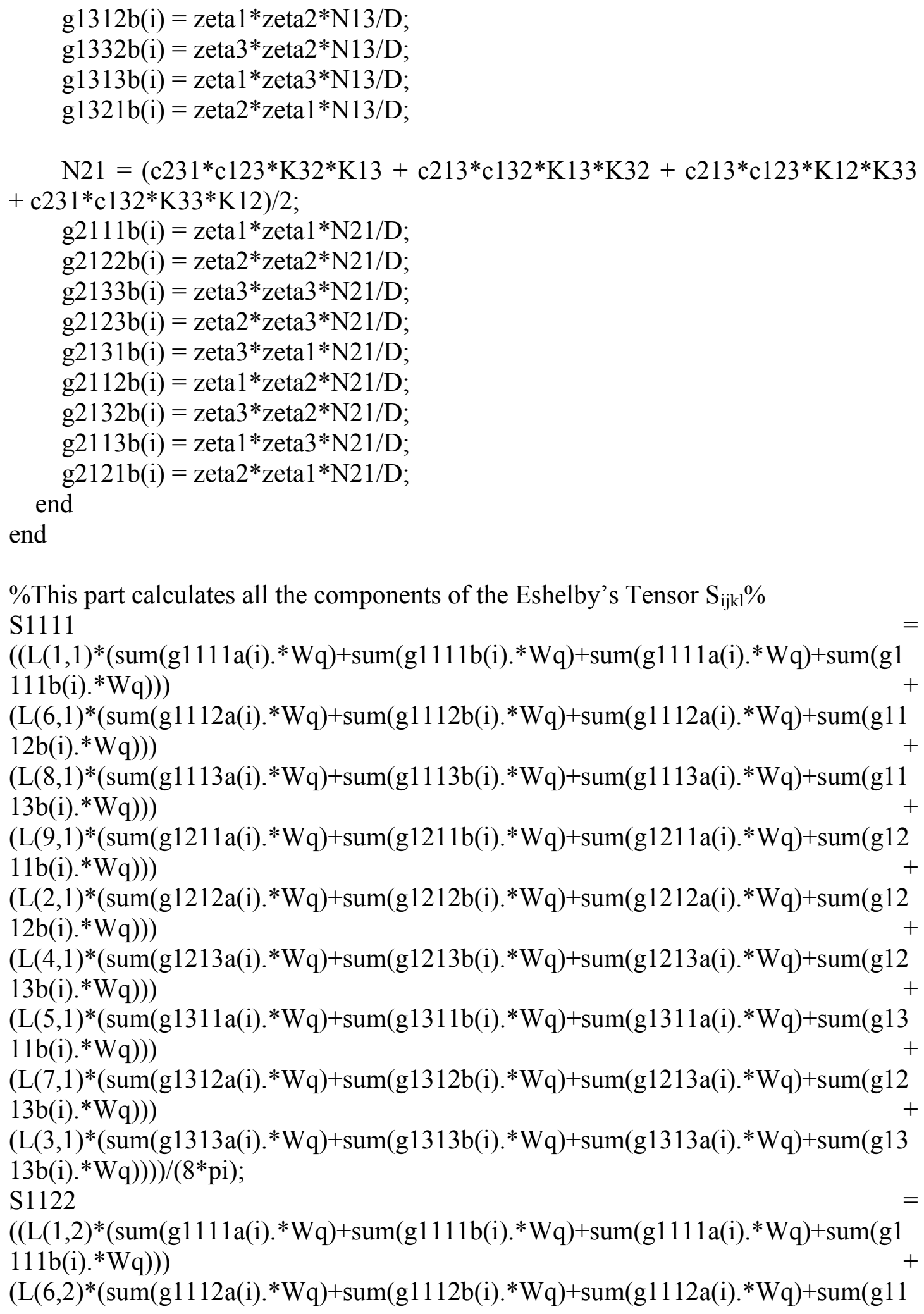


$12 \mathrm{~b}(\mathrm{i}) . * \mathrm{Wq})))$

$\left(\mathrm{L}(8,2) *\left(\operatorname{sum}\left(\mathrm{g} 1113 \mathrm{a}(\mathrm{i}) .{ }^{*} \mathrm{Wq}\right)+\operatorname{sum}\left(\mathrm{g} 1113 \mathrm{~b}(\mathrm{i}) .{ }^{*} \mathrm{Wq}\right)+\operatorname{sum}\left(\mathrm{g} 1113 \mathrm{a}(\mathrm{i}) .{ }^{*} \mathrm{Wq}\right)+\operatorname{sum}(\mathrm{g} 11\right.\right.$

$13 \mathrm{~b}(\mathrm{i}) . * \mathrm{Wq}))$ )

$\left(\mathrm{L}(9,2) *\left(\operatorname{sum}\left(\mathrm{g} 1211 \mathrm{a}(\mathrm{i}) \cdot{ }^{*} \mathrm{Wq}\right)+\operatorname{sum}\left(\mathrm{g} 1211 \mathrm{~b}(\mathrm{i}) \cdot{ }^{*} \mathrm{Wq}\right)+\operatorname{sum}\left(\mathrm{g} 1211 \mathrm{a}(\mathrm{i}) \cdot{ }^{*} \mathrm{Wq}\right)+\operatorname{sum}(\mathrm{g} 12\right.\right.$

$11 \mathrm{~b}(\mathrm{i}) . * \mathrm{Wq}))$

$\left(\mathrm{L}(2,2) *\left(\operatorname{sum}\left(\mathrm{g} 1212 \mathrm{a}(\mathrm{i}) \cdot{ }^{*} \mathrm{Wq}\right)+\operatorname{sum}\left(\mathrm{g} 1212 \mathrm{~b}(\mathrm{i}) \cdot{ }^{*} \mathrm{Wq}\right)+\operatorname{sum}\left(\mathrm{g} 1212 \mathrm{a}(\mathrm{i}) \cdot{ }^{*} \mathrm{Wq}\right)+\operatorname{sum}(\mathrm{g} 12\right.\right.$

$12 \mathrm{~b}(\mathrm{i}) . * \mathrm{Wq})))$

$\left(\mathrm{L}(4,2) *\left(\operatorname{sum}\left(\mathrm{g} 1213 \mathrm{a}(\mathrm{i}) .{ }^{*} \mathrm{Wq}\right)+\operatorname{sum}\left(\mathrm{g} 1213 \mathrm{~b}(\mathrm{i}) .{ }^{*} \mathrm{Wq}\right)+\operatorname{sum}\left(\mathrm{g} 1213 \mathrm{a}(\mathrm{i}) .{ }^{*} \mathrm{Wq}\right)+\operatorname{sum}(\mathrm{g} 12\right.\right.$

$13 \mathrm{~b}(\mathrm{i}) . * \mathrm{Wq}))$

$\left(\mathrm{L}(5,2) *\left(\operatorname{sum}\left(\mathrm{g} 1311 \mathrm{a}(\mathrm{i}) .{ }^{*} \mathrm{Wq}\right)+\operatorname{sum}\left(\mathrm{g} 1311 \mathrm{~b}(\mathrm{i}) .{ }^{*} \mathrm{Wq}\right)+\operatorname{sum}\left(\mathrm{g} 1311 \mathrm{a}(\mathrm{i}) .{ }^{*} \mathrm{Wq}\right)+\operatorname{sum}(\mathrm{g} 13\right.\right.$

$11 \mathrm{~b}(\mathrm{i}) . * \mathrm{Wq})))$

$\left(\mathrm{L}(7,2) *\left(\operatorname{sum}\left(\mathrm{g} 1312 \mathrm{a}(\mathrm{i}) .{ }^{*} \mathrm{Wq}\right)+\operatorname{sum}\left(\mathrm{g} 1312 \mathrm{~b}(\mathrm{i}) \cdot{ }^{*} \mathrm{Wq}\right)+\operatorname{sum}\left(\mathrm{g} 1213 \mathrm{a}(\mathrm{i}) \cdot{ }^{*} \mathrm{Wq}\right)+\operatorname{sum}(\mathrm{g} 12\right.\right.$

$13 \mathrm{~b}(\mathrm{i}) \cdot * \mathrm{Wq}))$

$\left(\mathrm{L}(3,2) *\left(\operatorname{sum}\left(\mathrm{g} 1313 \mathrm{a}(\mathrm{i}) .{ }^{*} \mathrm{Wq}\right)+\operatorname{sum}\left(\mathrm{g} 1313 \mathrm{~b}(\mathrm{i}) \cdot{ }^{*} \mathrm{Wq}\right)+\operatorname{sum}\left(\mathrm{g} 1313 \mathrm{a}(\mathrm{i}) .{ }^{*} \mathrm{Wq}\right)+\operatorname{sum}(\mathrm{g} 13\right.\right.$

$13 \mathrm{~b}(\mathrm{i}) * \mathrm{Wq})))) /(8 * \mathrm{pi})$;

S1133

$\left(\left(\mathrm{L}(1,3) *\left(\operatorname{sum}\left(\mathrm{g} 1111 \mathrm{a}(\mathrm{i}) .{ }^{*} \mathrm{Wq}\right)+\operatorname{sum}\left(\mathrm{g} 1111 \mathrm{~b}(\mathrm{i}) .{ }^{*} \mathrm{Wq}\right)+\operatorname{sum}\left(\mathrm{g} 1111 \mathrm{a}(\mathrm{i}) .{ }^{*} \mathrm{Wq}\right)+\operatorname{sum}(\mathrm{g} 1\right.\right.\right.$ $111 \mathrm{~b}(\mathrm{i}) * \mathrm{Wq})))$

$\left(\mathrm{L}(6,3) *\left(\operatorname{sum}\left(\mathrm{g} 1112 \mathrm{a}(\mathrm{i}) .{ }^{*} \mathrm{Wq}\right)+\operatorname{sum}\left(\mathrm{g} 1112 \mathrm{~b}(\mathrm{i}) \cdot{ }^{*} \mathrm{Wq}\right)+\operatorname{sum}\left(\mathrm{g} 1112 \mathrm{a}(\mathrm{i}) .{ }^{*} \mathrm{Wq}\right)+\operatorname{sum}(\mathrm{g} 11\right.\right.$

$12 \mathrm{~b}(\mathrm{i}) . * \mathrm{Wq})))$

$\left(\mathrm{L}(8,3) *\left(\operatorname{sum}\left(\mathrm{g} 1113 \mathrm{a}(\mathrm{i}) .{ }^{*} \mathrm{Wq}\right)+\operatorname{sum}\left(\mathrm{g} 1113 \mathrm{~b}(\mathrm{i}) .{ }^{*} \mathrm{Wq}\right)+\operatorname{sum}\left(\mathrm{g} 1113 \mathrm{a}(\mathrm{i}) .{ }^{*} \mathrm{Wq}\right)+\operatorname{sum}(\mathrm{g} 11\right.\right.$

$13 \mathrm{~b}(\mathrm{i}) . * \mathrm{Wq})))$

$\left(\mathrm{L}(9,3) *\left(\operatorname{sum}(\mathrm{g} 1211 \mathrm{a}(\mathrm{i}) . * \mathrm{Wq})+\operatorname{sum}\left(\mathrm{g} 1211 \mathrm{~b}(\mathrm{i}) \cdot{ }^{*} \mathrm{Wq}\right)+\operatorname{sum}\left(\mathrm{g} 1211 \mathrm{a}(\mathrm{i}) \cdot{ }^{*} \mathrm{Wq}\right)+\operatorname{sum}(\mathrm{g} 12\right.\right.$

$11 \mathrm{~b}(\mathrm{i}) . * \mathrm{Wq})))$

$\left(\mathrm{L}(2,3) *\left(\operatorname{sum}\left(\mathrm{g} 1212 \mathrm{a}(\mathrm{i}) \cdot{ }^{*} \mathrm{Wq}\right)+\operatorname{sum}\left(\mathrm{g} 1212 \mathrm{~b}(\mathrm{i}) \cdot{ }^{*} \mathrm{Wq}\right)+\operatorname{sum}\left(\mathrm{g} 1212 \mathrm{a}(\mathrm{i}) \cdot{ }^{*} \mathrm{Wq}\right)+\operatorname{sum}(\mathrm{g} 12\right.\right.$

$12 \mathrm{~b}(\mathrm{i}) . * \mathrm{Wq})))$

$\left(\mathrm{L}(4,3) *\left(\operatorname{sum}\left(\mathrm{g} 1213 \mathrm{a}(\mathrm{i}) .{ }^{*} \mathrm{Wq}\right)+\operatorname{sum}\left(\mathrm{g} 1213 \mathrm{~b}(\mathrm{i}) .{ }^{*} \mathrm{Wq}\right)+\operatorname{sum}\left(\mathrm{g} 1213 \mathrm{a}(\mathrm{i}) .{ }^{*} \mathrm{Wq}\right)+\operatorname{sum}(\mathrm{g} 12\right.\right.$

$13 \mathrm{~b}(\mathrm{i}) . * \mathrm{Wq}))$

$\left(\mathrm{L}(5,3) *\left(\operatorname{sum}\left(\mathrm{g} 1311 \mathrm{a}(\mathrm{i}) .{ }^{*} \mathrm{Wq}\right)+\operatorname{sum}\left(\mathrm{g} 1311 \mathrm{~b}(\mathrm{i}) \cdot{ }^{*} \mathrm{Wq}\right)+\operatorname{sum}\left(\mathrm{g} 1311 \mathrm{a}(\mathrm{i}) .{ }^{*} \mathrm{Wq}\right)+\operatorname{sum}(\mathrm{g} 13\right.\right.$

$11 \mathrm{~b}(\mathrm{i}) . * \mathrm{Wq})))$

$\left(\mathrm{L}(7,3) *\left(\operatorname{sum}\left(\mathrm{g} 1312 \mathrm{a}(\mathrm{i}) .{ }^{*} \mathrm{Wq}\right)+\operatorname{sum}\left(\mathrm{g} 1312 \mathrm{~b}(\mathrm{i}) \cdot{ }^{*} \mathrm{Wq}\right)+\operatorname{sum}\left(\mathrm{g} 1213 \mathrm{a}(\mathrm{i}) .{ }^{*} \mathrm{Wq}\right)+\operatorname{sum}(\mathrm{g} 12\right.\right.$

$13 \mathrm{~b}(\mathrm{i}) \cdot * \mathrm{Wq})))$

$\left(\mathrm{L}(3,3) *\left(\operatorname{sum}\left(\mathrm{g} 1313 \mathrm{a}(\mathrm{i}) .{ }^{*} \mathrm{Wq}\right)+\operatorname{sum}\left(\mathrm{g} 1313 \mathrm{~b}(\mathrm{i}) .{ }^{*} \mathrm{Wq}\right)+\operatorname{sum}\left(\mathrm{g} 1313 \mathrm{a}(\mathrm{i}) .{ }^{*} \mathrm{Wq}\right)+\operatorname{sum}(\mathrm{g} 13\right.\right.$

$\left.\left.\left.\left.13 \mathrm{~b}(\mathrm{i}){ }^{*} \mathrm{Wq}\right)\right)\right)\right) /(8 * \mathrm{pi})$;

$\mathrm{S} 1123$

$\left(\left(\mathrm{L}(1,4) *\left(\operatorname{sum}\left(\mathrm{g} 1111 \mathrm{a}(\mathrm{i}) .{ }^{*} \mathrm{Wq}\right)+\operatorname{sum}\left(\mathrm{g} 1111 \mathrm{~b}(\mathrm{i}) .{ }^{*} \mathrm{Wq}\right)+\operatorname{sum}\left(\mathrm{g} 1111 \mathrm{a}(\mathrm{i}) .{ }^{*} \mathrm{Wq}\right)+\operatorname{sum}(\mathrm{g} 1\right.\right.\right.$ $111 \mathrm{~b}(\mathrm{i}) * \mathrm{Wq})))$

$\left(\mathrm{L}(6,4) *\left(\operatorname{sum}\left(\mathrm{g} 1112 \mathrm{a}(\mathrm{i}) .{ }^{*} \mathrm{Wq}\right)+\operatorname{sum}\left(\mathrm{g} 1112 \mathrm{~b}(\mathrm{i}) .{ }^{*} \mathrm{Wq}\right)+\operatorname{sum}\left(\mathrm{g} 1112 \mathrm{a}(\mathrm{i}) .{ }^{*} \mathrm{Wq}\right)+\operatorname{sum}(\mathrm{g} 11\right.\right.$

$\left.\left.\left.12 \mathrm{~b}(\mathrm{i}){ }^{*} \mathrm{Wq}\right)\right)\right)$

$\left(\mathrm{L}(8,4) *\left(\operatorname{sum}\left(\mathrm{g} 1113 \mathrm{a}(\mathrm{i}) .{ }^{*} \mathrm{Wq}\right)+\operatorname{sum}\left(\mathrm{g} 1113 \mathrm{~b}(\mathrm{i}) \cdot{ }^{*} \mathrm{Wq}\right)+\operatorname{sum}\left(\mathrm{g} 1113 \mathrm{a}(\mathrm{i}) .{ }^{*} \mathrm{Wq}\right)+\operatorname{sum}(\mathrm{g} 11\right.\right.$

$13 \mathrm{~b}(\mathrm{i}) . * \mathrm{Wq}))$

$\left(\mathrm{L}(9,4) *\left(\operatorname{sum}(\mathrm{g} 1211 \mathrm{a}(\mathrm{i}) . * \mathrm{Wq})+\operatorname{sum}\left(\mathrm{g} 1211 \mathrm{~b}(\mathrm{i}) \cdot{ }^{*} \mathrm{Wq}\right)+\operatorname{sum}\left(\mathrm{g} 1211 \mathrm{a}(\mathrm{i}) \cdot{ }^{*} \mathrm{Wq}\right)+\operatorname{sum}(\mathrm{g} 12\right.\right.$

$\left.\left.\left.11 \mathrm{~b}(\mathrm{i}) .{ }^{*} \mathrm{Wq}\right)\right)\right)$ 
$\left(\mathrm{L}(2,4) *\left(\operatorname{sum}\left(\mathrm{g} 1212 \mathrm{a}(\mathrm{i}) \cdot{ }^{*} \mathrm{Wq}\right)+\operatorname{sum}\left(\mathrm{g} 1212 \mathrm{~b}(\mathrm{i}) \cdot{ }^{*} \mathrm{Wq}\right)+\operatorname{sum}\left(\mathrm{g} 1212 \mathrm{a}(\mathrm{i}) \cdot{ }^{*} \mathrm{Wq}\right)+\operatorname{sum}(\mathrm{g} 12\right.\right.$ $12 \mathrm{~b}(\mathrm{i}) . * \mathrm{Wq}))$ ) $\left(\mathrm{L}(4,4) *\left(\operatorname{sum}(\mathrm{g} 1213 \mathrm{a}(\mathrm{i}) . * \mathrm{Wq})+\operatorname{sum}\left(\mathrm{g} 1213 \mathrm{~b}(\mathrm{i}) \cdot{ }^{*} \mathrm{Wq}\right)+\operatorname{sum}\left(\mathrm{g} 1213 \mathrm{a}(\mathrm{i}) \cdot{ }^{*} \mathrm{Wq}\right)+\operatorname{sum}(\mathrm{g} 12\right.\right.$ $13 \mathrm{~b}(\mathrm{i}) . * \mathrm{Wq}))$ $\left(\mathrm{L}(5,4) *\left(\operatorname{sum}\left(\mathrm{g} 1311 \mathrm{a}(\mathrm{i}) .{ }^{*} \mathrm{Wq}\right)+\operatorname{sum}\left(\mathrm{g} 1311 \mathrm{~b}(\mathrm{i}) .{ }^{*} \mathrm{Wq}\right)+\operatorname{sum}\left(\mathrm{g} 1311 \mathrm{a}(\mathrm{i}) .{ }^{*} \mathrm{Wq}\right)+\operatorname{sum}(\mathrm{g} 13\right.\right.$ $11 \mathrm{~b}(\mathrm{i}) . * \mathrm{Wq})))$ $\left(\mathrm{L}(7,4) *\left(\operatorname{sum}\left(\mathrm{g} 1312 \mathrm{a}(\mathrm{i}) .{ }^{*} \mathrm{Wq}\right)+\operatorname{sum}\left(\mathrm{g} 1312 \mathrm{~b}(\mathrm{i}) \cdot{ }^{*} \mathrm{Wq}\right)+\operatorname{sum}\left(\mathrm{g} 1213 \mathrm{a}(\mathrm{i}) .{ }^{*} \mathrm{Wq}\right)+\operatorname{sum}(\mathrm{g} 12\right.\right.$ $13 \mathrm{~b}(\mathrm{i}) . * \mathrm{Wq})))$ $\left(\mathrm{L}(3,4) *\left(\operatorname{sum}\left(\mathrm{g} 1313 \mathrm{a}(\mathrm{i}) .{ }^{*} \mathrm{Wq}\right)+\operatorname{sum}\left(\mathrm{g} 1313 \mathrm{~b}(\mathrm{i}) .{ }^{*} \mathrm{Wq}\right)+\operatorname{sum}\left(\mathrm{g} 1313 \mathrm{a}(\mathrm{i}) .{ }^{*} \mathrm{Wq}\right)+\operatorname{sum}(\mathrm{g} 13\right.\right.$ $\left.\left.\left.\left.13 \mathrm{~b}(\mathrm{i}){ }^{*} \mathrm{Wq}\right)\right)\right)\right) /(8 * \mathrm{pi})$;

S1131

$\left(\left(\mathrm{L}(1,5) *\left(\operatorname{sum}\left(\mathrm{g} 1111 \mathrm{a}(\mathrm{i}) .{ }^{*} \mathrm{Wq}\right)+\operatorname{sum}\left(\mathrm{g} 1111 \mathrm{~b}(\mathrm{i}) .{ }^{*} \mathrm{Wq}\right)+\operatorname{sum}\left(\mathrm{g} 1111 \mathrm{a}(\mathrm{i}) .{ }^{*} \mathrm{Wq}\right)+\operatorname{sum}(\mathrm{g} 1\right.\right.\right.$ $\left.\left.\left.111 \mathrm{~b}(\mathrm{i}) \cdot{ }^{*} \mathrm{Wq}\right)\right)\right)$ $\left(\mathrm{L}(6,5) *\left(\operatorname{sum}\left(\mathrm{g} 1112 \mathrm{a}(\mathrm{i}) .{ }^{*} \mathrm{Wq}\right)+\operatorname{sum}\left(\mathrm{g} 1112 \mathrm{~b}(\mathrm{i}) .{ }^{*} \mathrm{Wq}\right)+\operatorname{sum}(\mathrm{g} 1112 \mathrm{a}(\mathrm{i}) . * \mathrm{Wq})+\operatorname{sum}(\mathrm{g} 11\right.\right.$ $12 \mathrm{~b}(\mathrm{i}) . * \mathrm{Wq}))$ ) $\left(\mathrm{L}(8,5) *\left(\operatorname{sum}\left(\mathrm{g} 1113 \mathrm{a}(\mathrm{i}) .{ }^{*} \mathrm{Wq}\right)+\operatorname{sum}\left(\mathrm{g} 1113 \mathrm{~b}(\mathrm{i}) \cdot{ }^{*} \mathrm{Wq}\right)+\operatorname{sum}\left(\mathrm{g} 1113 \mathrm{a}(\mathrm{i}) \cdot{ }^{*} \mathrm{Wq}\right)+\operatorname{sum}(\mathrm{g} 11\right.\right.$ $13 \mathrm{~b}(\mathrm{i}) . * \mathrm{Wq})))$ $\left(\mathrm{L}(9,5) *\left(\operatorname{sum}\left(\mathrm{g} 1211 \mathrm{a}(\mathrm{i}) .{ }^{*} \mathrm{Wq}\right)+\operatorname{sum}\left(\mathrm{g} 1211 \mathrm{~b}(\mathrm{i}) .{ }^{*} \mathrm{Wq}\right)+\operatorname{sum}\left(\mathrm{g} 1211 \mathrm{a}(\mathrm{i}) \cdot{ }^{*} \mathrm{Wq}\right)+\operatorname{sum}(\mathrm{g} 12\right.\right.$ $11 \mathrm{~b}(\mathrm{i}) . * \mathrm{Wq})))$ $\left(\mathrm{L}(2,5) *\left(\operatorname{sum}\left(\mathrm{g} 1212 \mathrm{a}(\mathrm{i}) .{ }^{*} \mathrm{Wq}\right)+\operatorname{sum}\left(\mathrm{g} 1212 \mathrm{~b}(\mathrm{i}) .{ }^{*} \mathrm{Wq}\right)+\operatorname{sum}\left(\mathrm{g} 1212 \mathrm{a}(\mathrm{i}) .{ }^{*} \mathrm{Wq}\right)+\operatorname{sum}(\mathrm{g} 12\right.\right.$ $12 \mathrm{~b}(\mathrm{i}) . * \mathrm{Wq})))$ $\left(\mathrm{L}(4,5) *\left(\operatorname{sum}\left(\mathrm{g} 1213 \mathrm{a}(\mathrm{i}) .{ }^{*} \mathrm{Wq}\right)+\operatorname{sum}\left(\mathrm{g} 1213 \mathrm{~b}(\mathrm{i}) \cdot{ }^{*} \mathrm{Wq}\right)+\operatorname{sum}\left(\mathrm{g} 1213 \mathrm{a}(\mathrm{i}) \cdot{ }^{*} \mathrm{Wq}\right)+\operatorname{sum}(\mathrm{g} 12\right.\right.$ $13 \mathrm{~b}(\mathrm{i}) . * \mathrm{Wq}))$ $\left(\mathrm{L}(5,5) *\left(\operatorname{sum}\left(\mathrm{g} 1311 \mathrm{a}(\mathrm{i}) .{ }^{*} \mathrm{Wq}\right)+\operatorname{sum}\left(\mathrm{g} 1311 \mathrm{~b}(\mathrm{i}) \cdot{ }^{*} \mathrm{Wq}\right)+\operatorname{sum}\left(\mathrm{g} 1311 \mathrm{a}(\mathrm{i}) .{ }^{*} \mathrm{Wq}\right)+\operatorname{sum}(\mathrm{g} 13\right.\right.$ $11 \mathrm{~b}(\mathrm{i}) . * \mathrm{Wq})))$ $\left(\mathrm{L}(7,5) *\left(\operatorname{sum}\left(\mathrm{g} 1312 \mathrm{a}(\mathrm{i}) .{ }^{*} \mathrm{Wq}\right)+\operatorname{sum}\left(\mathrm{g} 1312 \mathrm{~b}(\mathrm{i}) .{ }^{*} \mathrm{Wq}\right)+\operatorname{sum}\left(\mathrm{g} 1213 \mathrm{a}(\mathrm{i}) .{ }^{*} \mathrm{Wq}\right)+\operatorname{sum}(\mathrm{g} 12\right.\right.$ $13 \mathrm{~b}(\mathrm{i}) . * \mathrm{Wq}))$ $\left(\mathrm{L}(3,5) *\left(\operatorname{sum}\left(\mathrm{g} 1313 \mathrm{a}(\mathrm{i}) .{ }^{*} \mathrm{Wq}\right)+\operatorname{sum}\left(\mathrm{g} 1313 \mathrm{~b}(\mathrm{i}) .{ }^{*} \mathrm{Wq}\right)+\operatorname{sum}\left(\mathrm{g} 1313 \mathrm{a}(\mathrm{i}) .{ }^{*} \mathrm{Wq}\right)+\operatorname{sum}(\mathrm{g} 13\right.\right.$ $13 \mathrm{~b}(\mathrm{i}) . * \mathrm{Wq})))) /(8 * \mathrm{pi})$;

$\mathrm{S} 1112$ $\left(\left(\mathrm{L}(1,6) *\left(\operatorname{sum}\left(\mathrm{g} 1111 \mathrm{a}(\mathrm{i}) .{ }^{*} \mathrm{Wq}\right)+\operatorname{sum}\left(\mathrm{g} 1111 \mathrm{~b}(\mathrm{i}) .{ }^{*} \mathrm{Wq}\right)+\operatorname{sum}\left(\mathrm{g} 1111 \mathrm{a}(\mathrm{i}) .{ }^{*} \mathrm{Wq}\right)+\operatorname{sum}(\mathrm{g} 1\right.\right.\right.$ $111 \mathrm{~b}(\mathrm{i}) * \mathrm{Wq})))$ $\left(\mathrm{L}(6,6) *\left(\operatorname{sum}\left(\mathrm{g} 1112 \mathrm{a}(\mathrm{i}) .{ }^{*} \mathrm{Wq}\right)+\operatorname{sum}\left(\mathrm{g} 1112 \mathrm{~b}(\mathrm{i}) \cdot{ }^{*} \mathrm{Wq}\right)+\operatorname{sum}\left(\mathrm{g} 1112 \mathrm{a}(\mathrm{i}) .{ }^{*} \mathrm{Wq}\right)+\operatorname{sum}(\mathrm{g} 11\right.\right.$ $12 \mathrm{~b}(\mathrm{i}) . * \mathrm{Wq})))$ $\left(\mathrm{L}(8,6) *\left(\operatorname{sum}\left(\mathrm{g} 1113 \mathrm{a}(\mathrm{i}) .{ }^{*} \mathrm{Wq}\right)+\operatorname{sum}\left(\mathrm{g} 1113 \mathrm{~b}(\mathrm{i}) .{ }^{*} \mathrm{Wq}\right)+\operatorname{sum}\left(\mathrm{g} 1113 \mathrm{a}(\mathrm{i}) .{ }^{*} \mathrm{Wq}\right)+\operatorname{sum}(\mathrm{g} 11\right.\right.$ $13 \mathrm{~b}(\mathrm{i}) . * \mathrm{Wq})))$ $\left(\mathrm{L}(9,6) *\left(\operatorname{sum}\left(\mathrm{g} 1211 \mathrm{a}(\mathrm{i}) .{ }^{*} \mathrm{Wq}\right)+\operatorname{sum}\left(\mathrm{g} 1211 \mathrm{~b}(\mathrm{i}) \cdot{ }^{*} \mathrm{Wq}\right)+\operatorname{sum}\left(\mathrm{g} 1211 \mathrm{a}(\mathrm{i}) \cdot{ }^{*} \mathrm{Wq}\right)+\operatorname{sum}(\mathrm{g} 12\right.\right.$ $11 \mathrm{~b}(\mathrm{i}) . * \mathrm{Wq})))$ $\left(\mathrm{L}(2,6) *\left(\operatorname{sum}\left(\mathrm{g} 1212 \mathrm{a}(\mathrm{i}) .{ }^{*} \mathrm{Wq}\right)+\operatorname{sum}\left(\mathrm{g} 1212 \mathrm{~b}(\mathrm{i}) \cdot{ }^{*} \mathrm{Wq}\right)+\operatorname{sum}\left(\mathrm{g} 1212 \mathrm{a}(\mathrm{i}) \cdot{ }^{*} \mathrm{Wq}\right)+\operatorname{sum}(\mathrm{g} 12\right.\right.$ $12 \mathrm{~b}(\mathrm{i}) . * \mathrm{Wq})))$ $\left(\mathrm{L}(4,6) *\left(\operatorname{sum}\left(\mathrm{g} 1213 \mathrm{a}(\mathrm{i}) .{ }^{*} \mathrm{Wq}\right)+\operatorname{sum}\left(\mathrm{g} 1213 \mathrm{~b}(\mathrm{i}) \cdot{ }^{*} \mathrm{Wq}\right)+\operatorname{sum}\left(\mathrm{g} 1213 \mathrm{a}(\mathrm{i}) \cdot{ }^{*} \mathrm{Wq}\right)+\operatorname{sum}(\mathrm{g} 12\right.\right.$ $\left.\left.\left.13 \mathrm{~b}(\mathrm{i}) \cdot{ }^{*} \mathrm{Wq}\right)\right)\right)$ $\left(\mathrm{L}(5,6) *\left(\operatorname{sum}\left(\mathrm{g} 1311 \mathrm{a}(\mathrm{i}) .{ }^{*} \mathrm{Wq}\right)+\operatorname{sum}\left(\mathrm{g} 1311 \mathrm{~b}(\mathrm{i}) \cdot{ }^{*} \mathrm{Wq}\right)+\operatorname{sum}\left(\mathrm{g} 1311 \mathrm{a}(\mathrm{i}) \cdot{ }^{*} \mathrm{Wq}\right)+\operatorname{sum}(\mathrm{g} 13\right.\right.$ 
$11 \mathrm{~b}(\mathrm{i}) . * \mathrm{Wq})))$

$\left(\mathrm{L}(7,6) *\left(\operatorname{sum}\left(\mathrm{g} 1312 \mathrm{a}(\mathrm{i}) \cdot{ }^{*} \mathrm{Wq}\right)+\operatorname{sum}\left(\mathrm{g} 1312 \mathrm{~b}(\mathrm{i}) .{ }^{*} \mathrm{Wq}\right)+\operatorname{sum}\left(\mathrm{g} 1213 \mathrm{a}(\mathrm{i}) .{ }^{*} \mathrm{Wq}\right)+\operatorname{sum}(\mathrm{g} 12\right.\right.$

$13 \mathrm{~b}(\mathrm{i}) \cdot * \mathrm{Wq}))$ )

$\left(\mathrm{L}(3,6) *\left(\operatorname{sum}\left(\mathrm{g} 1313 \mathrm{a}(\mathrm{i}) .{ }^{*} \mathrm{Wq}\right)+\operatorname{sum}\left(\mathrm{g} 1313 \mathrm{~b}(\mathrm{i}) .{ }^{*} \mathrm{Wq}\right)+\operatorname{sum}\left(\mathrm{g} 1313 \mathrm{a}(\mathrm{i}) .{ }^{*} \mathrm{Wq}\right)+\operatorname{sum}(\mathrm{g} 13\right.\right.$

$13 \mathrm{~b}(\mathrm{i}) *$ Wq $)))) /(8 * \mathrm{pi})$;

S1132

$\left(\left(\mathrm{L}(1,7) *\left(\operatorname{sum}\left(\mathrm{g} 1111 \mathrm{a}(\mathrm{i}) .{ }^{*} \mathrm{Wq}\right)+\operatorname{sum}\left(\mathrm{g} 1111 \mathrm{~b}(\mathrm{i}) .{ }^{*} \mathrm{Wq}\right)+\operatorname{sum}\left(\mathrm{g} 1111 \mathrm{a}(\mathrm{i}) \cdot{ }^{*} \mathrm{Wq}\right)+\operatorname{sum}(\mathrm{g} 1\right.\right.\right.$ $\left.\left.\left.111 \mathrm{~b}(\mathrm{i}) *{ }^{*} \mathrm{Wq}\right)\right)\right)$

$\left(\mathrm{L}(6,7) *\left(\operatorname{sum}\left(\mathrm{g} 1112 \mathrm{a}(\mathrm{i}) \cdot{ }^{*} \mathrm{Wq}\right)+\operatorname{sum}\left(\mathrm{g} 1112 \mathrm{~b}(\mathrm{i}) \cdot{ }^{*} \mathrm{Wq}\right)+\operatorname{sum}\left(\mathrm{g} 1112 \mathrm{a}(\mathrm{i}){ }^{*} \mathrm{Wq}\right)+\operatorname{sum}(\mathrm{g} 11\right.\right.$

$\left.\left.12 \mathrm{~b}(\mathrm{i}){ }^{*} \mathrm{Wq}\right)\right)$ )

$\left(\mathrm{L}(8,7) *\left(\operatorname{sum}\left(\mathrm{g} 1113 \mathrm{a}(\mathrm{i}) .{ }^{*} \mathrm{Wq}\right)+\operatorname{sum}\left(\mathrm{g} 1113 \mathrm{~b}(\mathrm{i}) .{ }^{*} \mathrm{Wq}\right)+\operatorname{sum}\left(\mathrm{g} 1113 \mathrm{a}(\mathrm{i}) .{ }^{*} \mathrm{Wq}\right)+\operatorname{sum}(\mathrm{g} 11\right.\right.$

$13 b(\mathrm{i}) * * \mathrm{Wq})))$

$\left(\mathrm{L}(9,7) *\left(\operatorname{sum}\left(\mathrm{g} 1211 \mathrm{a}(\mathrm{i}) .{ }^{*} \mathrm{Wq}\right)+\operatorname{sum}\left(\mathrm{g} 1211 \mathrm{~b}(\mathrm{i}) .{ }^{*} \mathrm{Wq}\right)+\operatorname{sum}\left(\mathrm{g} 1211 \mathrm{a}(\mathrm{i}) .{ }^{*} \mathrm{Wq}\right)+\operatorname{sum}(\mathrm{g} 12\right.\right.$

$\left.\left.\left.11 \mathrm{~b}(\mathrm{i}) *{ }^{*} \mathrm{Wq}\right)\right)\right)$

$\left(\mathrm{L}(2,7) *\left(\operatorname{sum}\left(\mathrm{g} 1212 \mathrm{a}(\mathrm{i}) .{ }^{*} \mathrm{Wq}\right)+\operatorname{sum}\left(\mathrm{g} 1212 \mathrm{~b}(\mathrm{i}) \cdot{ }^{*} \mathrm{Wq}\right)+\operatorname{sum}\left(\mathrm{g} 1212 \mathrm{a}(\mathrm{i}) \cdot{ }^{* W q}\right)+\operatorname{sum}(\mathrm{g} 12\right.\right.$

$\left.\left.\left.12 \mathrm{~b}(\mathrm{i}) \cdot{ }^{*} \mathrm{Wq}\right)\right)\right)$

$\left(\mathrm{L}(4,7) *\left(\operatorname{sum}\left(\mathrm{g} 1213 \mathrm{a}(\mathrm{i}) \cdot{ }^{*} \mathrm{Wq}\right)+\operatorname{sum}\left(\mathrm{g} 1213 \mathrm{~b}(\mathrm{i}) \cdot{ }^{*} \mathrm{Wq}\right)+\operatorname{sum}\left(\mathrm{g} 1213 \mathrm{a}(\mathrm{i}) .{ }^{*} \mathrm{Wq}\right)+\operatorname{sum}(\mathrm{g} 12\right.\right.$

$13 \mathrm{~b}(\mathrm{i}) . * \mathrm{Wq})))$

$\left(\mathrm{L}(5,7) *\left(\operatorname{sum}\left(\mathrm{g} 1311 \mathrm{a}(\mathrm{i}) \cdot{ }^{*} \mathrm{Wq}\right)+\operatorname{sum}\left(\mathrm{g} 1311 \mathrm{~b}(\mathrm{i}) .{ }^{*} \mathrm{Wq}\right)+\operatorname{sum}\left(\mathrm{g} 1311 \mathrm{a}(\mathrm{i}) \cdot{ }^{*} \mathrm{Wq}\right)+\operatorname{sum}(\mathrm{g} 13\right.\right.$

$\left.\left.\left.11 \mathrm{~b}(\mathrm{i}){ }^{*} \mathrm{Wq}\right)\right)\right)$

$\left(\mathrm{L}(7,7) *\left(\operatorname{sum}\left(\mathrm{g} 1312 \mathrm{a}(\mathrm{i}) \cdot{ }^{*} \mathrm{Wq}\right)+\operatorname{sum}\left(\mathrm{g} 1312 \mathrm{~b}(\mathrm{i}) \cdot{ }^{*} \mathrm{Wq}\right)+\operatorname{sum}\left(\mathrm{g} 1213 \mathrm{a}(\mathrm{i}) .{ }^{*} \mathrm{Wq}\right)+\operatorname{sum}(\mathrm{g} 12\right.\right.$

$13 \mathrm{~b}(\mathrm{i}) . * \mathrm{Wq})))$

$\left(\mathrm{L}(3,7) *\left(\operatorname{sum}\left(\mathrm{g} 1313 \mathrm{a}(\mathrm{i}) .{ }^{*} \mathrm{Wq}\right)+\operatorname{sum}\left(\mathrm{g} 1313 \mathrm{~b}(\mathrm{i}) .{ }^{*} \mathrm{Wq}\right)+\operatorname{sum}\left(\mathrm{g} 1313 \mathrm{a}(\mathrm{i}) .{ }^{*} \mathrm{Wq}\right)+\operatorname{sum}(\mathrm{g} 13\right.\right.$

$13 \mathrm{~b}(\mathrm{i}) *$ (Wq) $))) /(8 * \mathrm{pi})$;

$\mathrm{S} 1113$

$\left(\left(\mathrm{L}(1,8) *\left(\operatorname{sum}\left(\mathrm{g} 1111 \mathrm{a}(\mathrm{i}) \cdot{ }^{*} \mathrm{Wq}\right)+\operatorname{sum}\left(\mathrm{g} 1111 \mathrm{~b}(\mathrm{i}) \cdot{ }^{*} \mathrm{Wq}\right)+\operatorname{sum}\left(\mathrm{g} 1111 \mathrm{a}(\mathrm{i}) \cdot{ }^{* W q}\right)+\operatorname{sum}(\mathrm{g} 1\right.\right.\right.$

$111 \mathrm{~b}(\mathrm{i}) * \mathrm{Wq})))$

$\left(\mathrm{L}(6,8) *\left(\operatorname{sum}\left(\mathrm{g} 1112 \mathrm{a}(\mathrm{i}) \cdot{ }^{*} \mathrm{Wq}\right)+\operatorname{sum}\left(\mathrm{g} 1112 \mathrm{~b}(\mathrm{i}) \cdot{ }^{*} \mathrm{Wq}\right)+\operatorname{sum}\left(\mathrm{g} 1112 \mathrm{a}(\mathrm{i}) \cdot{ }^{*} \mathrm{Wq}\right)+\operatorname{sum}(\mathrm{g} 11\right.\right.$

$12 \mathrm{~b}(\mathrm{i}) . * \mathrm{Wq})))$

$\left(\mathrm{L}(8,8) *\left(\operatorname{sum}\left(\mathrm{g} 1113 \mathrm{a}(\mathrm{i}) .{ }^{*} \mathrm{Wq}\right)+\operatorname{sum}\left(\mathrm{g} 1113 \mathrm{~b}(\mathrm{i}) .{ }^{*} \mathrm{Wq}\right)+\operatorname{sum}\left(\mathrm{g} 1113 \mathrm{a}(\mathrm{i}) .{ }^{* W q}\right)+\operatorname{sum}(\mathrm{g} 11\right.\right.$

$13 \mathrm{~b}(\mathrm{i}) . * \mathrm{Wq})))$

$\left(\mathrm{L}(9,8) *\left(\operatorname{sum}\left(\mathrm{g} 1211 \mathrm{a}(\mathrm{i}) .{ }^{*} \mathrm{Wq}\right)+\operatorname{sum}\left(\mathrm{g} 1211 \mathrm{~b}(\mathrm{i}) .{ }^{*} \mathrm{Wq}\right)+\operatorname{sum}\left(\mathrm{g} 1211 \mathrm{a}(\mathrm{i}) .{ }^{*} \mathrm{Wq}\right)+\operatorname{sum}(\mathrm{g} 12\right.\right.$

$\left.\left.11 \mathrm{~b}(\mathrm{i}) \cdot{ }^{*} \mathrm{Wq}\right)\right)$ )

$\left(\mathrm{L}(2,8) *\left(\operatorname{sum}\left(\mathrm{g} 1212 \mathrm{a}(\mathrm{i}) \cdot{ }^{*} \mathrm{Wq}\right)+\operatorname{sum}\left(\mathrm{g} 1212 \mathrm{~b}(\mathrm{i}) \cdot{ }^{*} \mathrm{Wq}\right)+\operatorname{sum}\left(\mathrm{g} 1212 \mathrm{a}(\mathrm{i}) \cdot{ }^{*} \mathrm{Wq}\right)+\operatorname{sum}(\mathrm{g} 12\right.\right.$

$12 \mathrm{~b}(\mathrm{i}) \cdot * \mathrm{Wq}))$ )

$\left(\mathrm{L}(4,8) *\left(\operatorname{sum}\left(\mathrm{g} 1213 \mathrm{a}(\mathrm{i}) .{ }^{*} \mathrm{Wq}\right)+\operatorname{sum}\left(\mathrm{g} 1213 \mathrm{~b}(\mathrm{i}) .{ }^{*} \mathrm{Wq}\right)+\operatorname{sum}\left(\mathrm{g} 1213 \mathrm{a}(\mathrm{i}) .{ }^{* W q}\right)+\operatorname{sum}(\mathrm{g} 12\right.\right.$ $\left.\left.13 \mathrm{~b}(\mathrm{i}) \cdot{ }^{*} \mathrm{Wq}\right)\right)$

$\left(\mathrm{L}(5,8) *\left(\operatorname{sum}\left(\mathrm{g} 1311 \mathrm{a}(\mathrm{i}) .{ }^{*} \mathrm{Wq}\right)+\operatorname{sum}\left(\mathrm{g} 1311 \mathrm{~b}(\mathrm{i}) .{ }^{*} \mathrm{Wq}\right)+\operatorname{sum}\left(\mathrm{g} 1311 \mathrm{a}(\mathrm{i}){ }^{*} \mathrm{Wq}\right)+\operatorname{sum}(\mathrm{g} 13\right.\right.$

$\left.\left.\left.11 \mathrm{~b}(\mathrm{i}){ }^{*} \mathrm{Wq}\right)\right)\right)$

$\left(\mathrm{L}(7,8) *\left(\operatorname{sum}\left(\mathrm{g} 1312 \mathrm{a}(\mathrm{i}) \cdot{ }^{*} \mathrm{Wq}\right)+\operatorname{sum}\left(\mathrm{g} 1312 \mathrm{~b}(\mathrm{i}) \cdot{ }^{*} \mathrm{Wq}\right)+\operatorname{sum}\left(\mathrm{g} 1213 \mathrm{a}(\mathrm{i}) \cdot{ }^{*} \mathrm{Wq}\right)+\operatorname{sum}(\mathrm{g} 12\right.\right.$

$13 \mathrm{~b}(\mathrm{i}) . * \mathrm{Wq}))$ )

$\left(\mathrm{L}(3,8) *\left(\operatorname{sum}\left(\mathrm{g} 1313 \mathrm{a}(\mathrm{i}) .{ }^{*} \mathrm{Wq}\right)+\operatorname{sum}\left(\mathrm{g} 1313 \mathrm{~b}(\mathrm{i}) .{ }^{*} \mathrm{Wq}\right)+\operatorname{sum}\left(\mathrm{g} 1313 \mathrm{a}(\mathrm{i}) .{ }^{*} \mathrm{Wq}\right)+\operatorname{sum}(\mathrm{g} 13\right.\right.$

$13 \mathrm{~b}(\mathrm{i}) *$ Wq $)))) /(8 * \mathrm{pi})$; 
S1121

$\left(\left(\mathrm{L}(1,9) *\left(\operatorname{sum}\left(\mathrm{g} 1111 \mathrm{a}(\mathrm{i}) .{ }^{*} \mathrm{Wq}\right)+\operatorname{sum}\left(\mathrm{g} 1111 \mathrm{~b}(\mathrm{i}) .{ }^{*} \mathrm{Wq}\right)+\operatorname{sum}\left(\mathrm{g} 1111 \mathrm{a}(\mathrm{i}) .{ }^{*} \mathrm{Wq}\right)+\operatorname{sum}(\mathrm{g} 1\right.\right.\right.$ $\left.\left.\left.111 \mathrm{~b}(\mathrm{i}) *{ }^{*} \mathrm{Wq}\right)\right)\right)$

$\left(\mathrm{L}(6,9) *\left(\operatorname{sum}\left(\mathrm{g} 1112 \mathrm{a}(\mathrm{i}) \cdot{ }^{*} \mathrm{Wq}\right)+\operatorname{sum}\left(\mathrm{g} 1112 \mathrm{~b}(\mathrm{i}) \cdot{ }^{*} \mathrm{Wq}\right)+\operatorname{sum}\left(\mathrm{g} 1112 \mathrm{a}(\mathrm{i}) .{ }^{*} \mathrm{Wq}\right)+\operatorname{sum}(\mathrm{g} 11\right.\right.$ $12 \mathrm{~b}(\mathrm{i}) \cdot * \mathrm{Wq}))$ )

$\left(\mathrm{L}(8,9) *\left(\operatorname{sum}\left(\mathrm{g} 1113 \mathrm{a}(\mathrm{i}) .{ }^{*} \mathrm{Wq}\right)+\operatorname{sum}\left(\mathrm{g} 1113 \mathrm{~b}(\mathrm{i}) .{ }^{*} \mathrm{Wq}\right)+\operatorname{sum}\left(\mathrm{g} 1113 \mathrm{a}(\mathrm{i}) .{ }^{*} \mathrm{Wq}\right)+\operatorname{sum}(\mathrm{g} 11\right.\right.$ $\left.\left.\left.13 \mathrm{~b}(\mathrm{i}) *{ }^{*} \mathrm{Wq}\right)\right)\right)$

$\left(\mathrm{L}(9,9) *\left(\operatorname{sum}\left(\mathrm{g} 1211 \mathrm{a}(\mathrm{i}) \cdot{ }^{*} \mathrm{Wq}\right)+\operatorname{sum}\left(\mathrm{g} 1211 \mathrm{~b}(\mathrm{i}) .{ }^{*} \mathrm{Wq}\right)+\operatorname{sum}\left(\mathrm{g} 1211 \mathrm{a}(\mathrm{i}) .{ }^{*} \mathrm{Wq}\right)+\operatorname{sum}(\mathrm{g} 12\right.\right.$ $\left.\left.\left.11 \mathrm{~b}(\mathrm{i}) \cdot{ }^{*} \mathrm{Wq}\right)\right)\right)$

$\left(\mathrm{L}(2,9) *\left(\operatorname{sum}(\mathrm{g} 1212 \mathrm{a}(\mathrm{i}) . * \mathrm{Wq})+\operatorname{sum}\left(\mathrm{g} 1212 \mathrm{~b}(\mathrm{i}) .{ }^{*} \mathrm{Wq}\right)+\operatorname{sum}\left(\mathrm{g} 1212 \mathrm{a}(\mathrm{i}) .{ }^{*} \mathrm{Wq}\right)+\operatorname{sum}(\mathrm{g} 12\right.\right.$ $\left.\left.\left.12 \mathrm{~b}(\mathrm{i}){ }^{*} \mathrm{Wq}\right)\right)\right)$

$\left(\mathrm{L}(4,9) *\left(\operatorname{sum}\left(\mathrm{g} 1213 \mathrm{a}(\mathrm{i}) \cdot{ }^{*} \mathrm{Wq}\right)+\operatorname{sum}\left(\mathrm{g} 1213 \mathrm{~b}(\mathrm{i}) \cdot{ }^{*} \mathrm{Wq}\right)+\operatorname{sum}\left(\mathrm{g} 1213 \mathrm{a}(\mathrm{i}){ }^{*} \mathrm{Wq}\right)+\operatorname{sum}(\mathrm{g} 12\right.\right.$ $13 \mathrm{~b}(\mathrm{i}) . * \mathrm{Wq})))$

$\left(\mathrm{L}(5,9) *\left(\operatorname{sum}\left(\mathrm{g} 1311 \mathrm{a}(\mathrm{i}) .{ }^{*} \mathrm{Wq}\right)+\operatorname{sum}\left(\mathrm{g} 1311 \mathrm{~b}(\mathrm{i}) .{ }^{*} \mathrm{Wq}\right)+\operatorname{sum}\left(\mathrm{g} 1311 \mathrm{a}(\mathrm{i}) .{ }^{* W \mathrm{~W}}\right)+\operatorname{sum}(\mathrm{g} 13\right.\right.$ $11 \mathrm{~b}(\mathrm{i}) . * \mathrm{Wq})))$

$\left(\mathrm{L}(7,9) *\left(\operatorname{sum}\left(\mathrm{g} 1312 \mathrm{a}(\mathrm{i}) .{ }^{*} \mathrm{Wq}\right)+\operatorname{sum}\left(\mathrm{g} 1312 \mathrm{~b}(\mathrm{i}) .{ }^{*} \mathrm{Wq}\right)+\operatorname{sum}\left(\mathrm{g} 1213 \mathrm{a}(\mathrm{i}){ }^{*} \mathrm{Wq}\right)+\operatorname{sum}(\mathrm{g} 12\right.\right.$ $13 \mathrm{~b}(\mathrm{i}) . * \mathrm{Wq})))$

$\left(\mathrm{L}(3,9) *\left(\operatorname{sum}\left(\mathrm{g} 1313 \mathrm{a}(\mathrm{i}) \cdot{ }^{*} \mathrm{Wq}\right)+\operatorname{sum}\left(\mathrm{g} 1313 \mathrm{~b}(\mathrm{i}) \cdot{ }^{*} \mathrm{Wq}\right)+\operatorname{sum}\left(\mathrm{g} 1313 \mathrm{a}(\mathrm{i}){ }^{*} \mathrm{Wq}\right)+\operatorname{sum}(\mathrm{g} 13\right.\right.$ $\left.\left.\left.\left.13 \mathrm{~b}(\mathrm{i}) .{ }^{*} \mathrm{Wq}\right)\right)\right)\right) /(8 * \mathrm{pi})$;

$\mathrm{S} 2211$

$\left(\left(\mathrm{L}(1,1) *\left(\operatorname{sum}\left(\mathrm{g} 2121 \mathrm{a}(\mathrm{i}) .{ }^{*} \mathrm{Wq}\right)+\operatorname{sum}\left(\mathrm{g} 2121 \mathrm{~b}(\mathrm{i}) \cdot{ }^{*} \mathrm{Wq}\right)+\operatorname{sum}\left(\mathrm{g} 2121 \mathrm{a}(\mathrm{i}) .{ }^{*} \mathrm{Wq}\right)+\operatorname{sum}(\mathrm{g} 2\right.\right.\right.$ $121 \mathrm{~b}(\mathrm{i}) \cdot * \mathrm{Wq})))$

$\left(\mathrm{L}(6,1) *\left(\operatorname{sum}\left(\mathrm{g} 2122 \mathrm{a}(\mathrm{i}) .{ }^{*} \mathrm{Wq}\right)+\operatorname{sum}\left(\mathrm{g} 2122 \mathrm{~b}(\mathrm{i}) .{ }^{*} \mathrm{Wq}\right)+\operatorname{sum}\left(\mathrm{g} 2122 \mathrm{a}(\mathrm{i}) .{ }^{*} \mathrm{Wq}\right)+\operatorname{sum}(\mathrm{g} 21\right.\right.$ $\left.\left.\left.22 \mathrm{~b}(\mathrm{i}){ }^{*} \mathrm{Wq}\right)\right)\right)$

$\left(\mathrm{L}(8,1) *\left(\operatorname{sum}\left(\mathrm{g} 2123 \mathrm{a}(\mathrm{i}) \cdot{ }^{*} \mathrm{Wq}\right)+\operatorname{sum}\left(\mathrm{g} 2123 \mathrm{~b}(\mathrm{i}) \cdot{ }^{*} \mathrm{Wq}\right)+\operatorname{sum}\left(\mathrm{g} 2123 \mathrm{a}(\mathrm{i}) \cdot{ }^{* \mathrm{Wq}}\right)+\operatorname{sum}(\mathrm{g} 21\right.\right.$ $\left.\left.\left.23 \mathrm{~b}(\mathrm{i}) *{ }^{*} \mathrm{Wq}\right)\right)\right)$

$\left(\mathrm{L}(9,1) *\left(\operatorname{sum}\left(\mathrm{g} 2221 \mathrm{a}(\mathrm{i}) .{ }^{*} \mathrm{Wq}\right)+\operatorname{sum}\left(\mathrm{g} 2221 \mathrm{~b}(\mathrm{i}) .{ }^{*} \mathrm{Wq}\right)+\operatorname{sum}\left(\mathrm{g} 2221 \mathrm{a}(\mathrm{i}){ }^{*} \mathrm{Wq}\right)+\operatorname{sum}(\mathrm{g} 22\right.\right.$ $21 \mathrm{~b}(\mathrm{i}) . * \mathrm{Wq})))$

$\left(\mathrm{L}(2,1) *\left(\operatorname{sum}\left(\mathrm{g} 2222 \mathrm{a}(\mathrm{i}) .{ }^{*} \mathrm{Wq}\right)+\operatorname{sum}\left(\mathrm{g} 2222 \mathrm{~b}(\mathrm{i}) .{ }^{*} \mathrm{Wq}\right)+\operatorname{sum}\left(\mathrm{g} 2222 \mathrm{a}(\mathrm{i}){ }^{*} \mathrm{Wq}\right)+\operatorname{sum}(\mathrm{g} 22\right.\right.$ $\left.\left.\left.22 \mathrm{~b}(\mathrm{i}) *{ }^{*} \mathrm{Wq}\right)\right)\right)$

$\left(\mathrm{L}(4,1) *\left(\operatorname{sum}\left(\mathrm{g} 2223 \mathrm{a}(\mathrm{i}) .{ }^{*} \mathrm{Wq}\right)+\operatorname{sum}\left(\mathrm{g} 2223 \mathrm{~b}(\mathrm{i}) \cdot{ }^{*} \mathrm{Wq}\right)+\operatorname{sum}\left(\mathrm{g} 2223 \mathrm{a}(\mathrm{i}){ }^{*} \mathrm{Wq}\right)+\operatorname{sum}(\mathrm{g} 22\right.\right.$ $23 \mathrm{~b}(\mathrm{i}) \cdot * \mathrm{Wq})))$

$\left(\mathrm{L}(5,1) *\left(\operatorname{sum}\left(\mathrm{g} 2321 \mathrm{a}(\mathrm{i}) .{ }^{*} \mathrm{Wq}\right)+\operatorname{sum}\left(\mathrm{g} 2321 \mathrm{~b}(\mathrm{i}) .{ }^{*} \mathrm{Wq}\right)+\operatorname{sum}\left(\mathrm{g} 2321 \mathrm{a}(\mathrm{i}) .{ }^{*} \mathrm{Wq}\right)+\operatorname{sum}(\mathrm{g} 23\right.\right.$ $21 \mathrm{~b}(\mathrm{i}) . * \mathrm{Wq})))$

$\left(\mathrm{L}(7,1) *\left(\operatorname{sum}\left(\mathrm{g} 2322 \mathrm{a}(\mathrm{i}) \cdot{ }^{*} \mathrm{Wq}\right)+\operatorname{sum}\left(\mathrm{g} 2322 \mathrm{~b}(\mathrm{i}) \cdot{ }^{*} \mathrm{Wq}\right)+\operatorname{sum}\left(\mathrm{g} 2322 \mathrm{a}(\mathrm{i}) \cdot{ }^{* \mathrm{Wq}}\right)+\operatorname{sum}(\mathrm{g} 23\right.\right.$ $\left.\left.\left.22 \mathrm{~b}(\mathrm{i}) *{ }^{*} \mathrm{Wq}\right)\right)\right)$

$\left(\mathrm{L}(3,1) *\left(\operatorname{sum}\left(\mathrm{g} 2323 \mathrm{a}(\mathrm{i}) .{ }^{*} \mathrm{Wq}\right)+\operatorname{sum}\left(\mathrm{g} 2323 \mathrm{~b}(\mathrm{i}) .{ }^{*} \mathrm{Wq}\right)+\operatorname{sum}\left(\mathrm{g} 2323 \mathrm{a}(\mathrm{i}) .{ }^{*} \mathrm{Wq}\right)+\operatorname{sum}(\mathrm{g} 23\right.\right.$ $\left.\left.\left.\left.23 \mathrm{~b}(\mathrm{i}){ }^{*} \mathrm{Wq}\right)\right)\right)\right) /\left(8^{*} \mathrm{pi}\right)$;

$\mathrm{S} 2222$

$\left(\left(\mathrm{L}(1,2) *\left(\operatorname{sum}\left(\mathrm{g} 2121 \mathrm{a}(\mathrm{i}) .{ }^{*} \mathrm{Wq}\right)+\operatorname{sum}\left(\mathrm{g} 2121 \mathrm{~b}(\mathrm{i}) .{ }^{*} \mathrm{Wq}\right)+\operatorname{sum}\left(\mathrm{g} 2121 \mathrm{a}(\mathrm{i}) \cdot{ }^{*} \mathrm{Wq}\right)+\operatorname{sum}(\mathrm{g} 2\right.\right.\right.$ 121b(i).*Wq)))

$\left(\mathrm{L}(6,2) *\left(\operatorname{sum}\left(\mathrm{g} 2122 \mathrm{a}(\mathrm{i}) .{ }^{*} \mathrm{Wq}\right)+\operatorname{sum}\left(\mathrm{g} 2122 \mathrm{~b}(\mathrm{i}) .{ }^{*} \mathrm{Wq}\right)+\operatorname{sum}\left(\mathrm{g} 2122 \mathrm{a}(\mathrm{i}){ }^{*} \mathrm{Wq}\right)+\operatorname{sum}(\mathrm{g} 21\right.\right.$ 
$22 \mathrm{~b}(\mathrm{i}) \cdot * \mathrm{Wq})))$

$\left(\mathrm{L}(8,2) *\left(\operatorname{sum}(\mathrm{g} 2123 \mathrm{a}(\mathrm{i}) . * \mathrm{Wq})+\operatorname{sum}\left(\mathrm{g} 2123 \mathrm{~b}(\mathrm{i}) \cdot{ }^{*} \mathrm{Wq}\right)+\operatorname{sum}\left(\mathrm{g} 2123 \mathrm{a}(\mathrm{i}) \cdot{ }^{*} \mathrm{Wq}\right)+\operatorname{sum}(\mathrm{g} 21\right.\right.$

$\left.\left.\left.23 \mathrm{~b}(\mathrm{i}) *{ }^{*} \mathrm{Wq}\right)\right)\right)$

$\left(\mathrm{L}(9,2) *\left(\operatorname{sum}\left(\mathrm{g} 2221 \mathrm{a}(\mathrm{i}) .{ }^{*} \mathrm{Wq}\right)+\operatorname{sum}(\mathrm{g} 2221 \mathrm{~b}(\mathrm{i}) . * \mathrm{Wq})+\operatorname{sum}\left(\mathrm{g} 2221 \mathrm{a}(\mathrm{i}) .{ }^{*} \mathrm{Wq}\right)+\operatorname{sum}(\mathrm{g} 22\right.\right.$

$\left.\left.\left.21 \mathrm{~b}(\mathrm{i}) \cdot{ }^{*} \mathrm{Wq}\right)\right)\right)$

$\left(\mathrm{L}(2,2) *\left(\operatorname{sum}(\mathrm{g} 2222 \mathrm{a}(\mathrm{i}) . * \mathrm{Wq})+\operatorname{sum}\left(\mathrm{g} 2222 \mathrm{~b}(\mathrm{i}) \cdot{ }^{*} \mathrm{Wq}\right)+\operatorname{sum}\left(\mathrm{g} 2222 \mathrm{a}(\mathrm{i}) \cdot{ }^{*} \mathrm{Wq}\right)+\operatorname{sum}(\mathrm{g} 22\right.\right.$

$22 \mathrm{~b}(\mathrm{i}) . * \mathrm{Wq})))$

$\left(\mathrm{L}(4,2) *\left(\operatorname{sum}(\mathrm{g} 2223 \mathrm{a}(\mathrm{i}) . * \mathrm{Wq})+\operatorname{sum}\left(\mathrm{g} 2223 \mathrm{~b}(\mathrm{i}) \cdot{ }^{*} \mathrm{Wq}\right)+\operatorname{sum}\left(\mathrm{g} 2223 \mathrm{a}(\mathrm{i}) .{ }^{*} \mathrm{Wq}\right)+\operatorname{sum}(\mathrm{g} 22\right.\right.$

$23 \mathrm{~b}(\mathrm{i}) . * \mathrm{Wq}))$

$\left(\mathrm{L}(5,2) *\left(\operatorname{sum}\left(\mathrm{g} 2321 \mathrm{a}(\mathrm{i}) .{ }^{*} \mathrm{Wq}\right)+\operatorname{sum}\left(\mathrm{g} 2321 \mathrm{~b}(\mathrm{i}) .{ }^{*} \mathrm{Wq}\right)+\operatorname{sum}\left(\mathrm{g} 2321 \mathrm{a}(\mathrm{i}) .{ }^{*} \mathrm{Wq}\right)+\operatorname{sum}(\mathrm{g} 23\right.\right.$

$21 \mathrm{~b}(\mathrm{i}) . * \mathrm{Wq})))$

$\left(\mathrm{L}(7,2) *\left(\operatorname{sum}\left(\mathrm{g} 2322 \mathrm{a}(\mathrm{i}) .{ }^{*} \mathrm{Wq}\right)+\operatorname{sum}\left(\mathrm{g} 2322 \mathrm{~b}(\mathrm{i}) .{ }^{*} \mathrm{Wq}\right)+\operatorname{sum}\left(\mathrm{g} 2322 \mathrm{a}(\mathrm{i}) .{ }^{*} \mathrm{Wq}\right)+\operatorname{sum}(\mathrm{g} 23\right.\right.$

$\left.\left.\left.22 \mathrm{~b}(\mathrm{i}) \cdot{ }^{*} \mathrm{Wq}\right)\right)\right)$

$\left(\mathrm{L}(3,2) *\left(\operatorname{sum}\left(\mathrm{g} 2323 \mathrm{a}(\mathrm{i}) .{ }^{*} \mathrm{Wq}\right)+\operatorname{sum}\left(\mathrm{g} 2323 \mathrm{~b}(\mathrm{i}) \cdot{ }^{*} \mathrm{Wq}\right)+\operatorname{sum}\left(\mathrm{g} 2323 \mathrm{a}(\mathrm{i}) .{ }^{*} \mathrm{Wq}\right)+\operatorname{sum}(\mathrm{g} 23\right.\right.$

$\left.\left.\left.\left.23 \mathrm{~b}(\mathrm{i}) \cdot{ }^{*} \mathrm{Wq}\right)\right)\right)\right) /(8 * \mathrm{pi})$;

$\mathrm{S} 2233$

$((\mathrm{L}(1,3) *(\operatorname{sum}(\mathrm{g} 2121 \mathrm{a}(\mathrm{i}) . * \mathrm{Wq})+\operatorname{sum}(\mathrm{g} 2121 \mathrm{~b}(\mathrm{i}) . * \mathrm{Wq})+\operatorname{sum}(\mathrm{g} 2121 \mathrm{a}(\mathrm{i}) . * \mathrm{Wq})+\operatorname{sum}(\mathrm{g} 2$ $\left.\left.\left.121 \mathrm{~b}(\mathrm{i}) *{ }^{*} \mathrm{Wq}\right)\right)\right)$

$\left(\mathrm{L}(6,3) *\left(\operatorname{sum}\left(\mathrm{g} 2122 \mathrm{a}(\mathrm{i}) .{ }^{*} \mathrm{Wq}\right)+\operatorname{sum}\left(\mathrm{g} 2122 \mathrm{~b}(\mathrm{i}) .{ }^{*} \mathrm{Wq}\right)+\operatorname{sum}\left(\mathrm{g} 2122 \mathrm{a}(\mathrm{i}) .{ }^{*} \mathrm{Wq}\right)+\operatorname{sum}(\mathrm{g} 21\right.\right.$ $22 \mathrm{~b}(\mathrm{i}) . * \mathrm{Wq})))$

$\left(\mathrm{L}(8,3) *\left(\operatorname{sum}\left(\mathrm{g} 2123 \mathrm{a}(\mathrm{i}) .{ }^{*} \mathrm{Wq}\right)+\operatorname{sum}\left(\mathrm{g} 2123 \mathrm{~b}(\mathrm{i}) .{ }^{*} \mathrm{Wq}\right)+\operatorname{sum}\left(\mathrm{g} 2123 \mathrm{a}(\mathrm{i}) .{ }^{*} \mathrm{Wq}\right)+\operatorname{sum}(\mathrm{g} 21\right.\right.$

$23 \mathrm{~b}(\mathrm{i}) . * \mathrm{Wq})))$

$\left(\mathrm{L}(9,3) *\left(\operatorname{sum}\left(\mathrm{g} 2221 \mathrm{a}(\mathrm{i}) .{ }^{*} \mathrm{Wq}\right)+\operatorname{sum}\left(\mathrm{g} 2221 \mathrm{~b}(\mathrm{i}) .{ }^{*} \mathrm{Wq}\right)+\operatorname{sum}\left(\mathrm{g} 2221 \mathrm{a}(\mathrm{i}) .{ }^{*} \mathrm{Wq}\right)+\operatorname{sum}(\mathrm{g} 22\right.\right.$

$21 \mathrm{~b}(\mathrm{i}) . * \mathrm{Wq}))$

$\left(\mathrm{L}(2,3) *\left(\operatorname{sum}\left(\mathrm{g} 2222 \mathrm{a}(\mathrm{i}) .{ }^{*} \mathrm{Wq}\right)+\operatorname{sum}\left(\mathrm{g} 2222 \mathrm{~b}(\mathrm{i}) \cdot{ }^{*} \mathrm{Wq}\right)+\operatorname{sum}\left(\mathrm{g} 2222 \mathrm{a}(\mathrm{i}) \cdot{ }^{*} \mathrm{Wq}\right)+\operatorname{sum}(\mathrm{g} 22\right.\right.$

$22 \mathrm{~b}(\mathrm{i}) . * \mathrm{Wq})))$

$\left(\mathrm{L}(4,3) *\left(\operatorname{sum}\left(\mathrm{g} 2223 \mathrm{a}(\mathrm{i}) .{ }^{*} \mathrm{Wq}\right)+\operatorname{sum}\left(\mathrm{g} 2223 \mathrm{~b}(\mathrm{i}) .{ }^{*} \mathrm{Wq}\right)+\operatorname{sum}\left(\mathrm{g} 2223 \mathrm{a}(\mathrm{i}) .{ }^{*} \mathrm{Wq}\right)+\operatorname{sum}(\mathrm{g} 22\right.\right.$

$23 \mathrm{~b}(\mathrm{i}) . * \mathrm{Wq}))$

$\left(\mathrm{L}(5,3) *\left(\operatorname{sum}\left(\mathrm{g} 2321 \mathrm{a}(\mathrm{i}) .{ }^{*} \mathrm{Wq}\right)+\operatorname{sum}\left(\mathrm{g} 2321 \mathrm{~b}(\mathrm{i}) \cdot{ }^{*} \mathrm{Wq}\right)+\operatorname{sum}\left(\mathrm{g} 2321 \mathrm{a}(\mathrm{i}) .{ }^{*} \mathrm{Wq}\right)+\operatorname{sum}(\mathrm{g} 23\right.\right.$

$21 \mathrm{~b}(\mathrm{i}) . * \mathrm{Wq})))$

$\left(\mathrm{L}(7,3) *\left(\operatorname{sum}\left(\mathrm{g} 2322 \mathrm{a}(\mathrm{i}) .{ }^{*} \mathrm{Wq}\right)+\operatorname{sum}\left(\mathrm{g} 2322 \mathrm{~b}(\mathrm{i}) .{ }^{*} \mathrm{Wq}\right)+\operatorname{sum}(\mathrm{g} 2322 \mathrm{a}(\mathrm{i}) . * \mathrm{Wq})+\operatorname{sum}(\mathrm{g} 23\right.\right.$

$22 \mathrm{~b}(\mathrm{i}) \cdot * \mathrm{Wq})))$

$\left(\mathrm{L}(3,3) *\left(\operatorname{sum}\left(\mathrm{g} 2323 \mathrm{a}(\mathrm{i}) .{ }^{*} \mathrm{Wq}\right)+\operatorname{sum}\left(\mathrm{g} 2323 \mathrm{~b}(\mathrm{i}) \cdot{ }^{*} \mathrm{Wq}\right)+\operatorname{sum}\left(\mathrm{g} 2323 \mathrm{a}(\mathrm{i}) .{ }^{*} \mathrm{Wq}\right)+\operatorname{sum}(\mathrm{g} 23\right.\right.$

$23 \mathrm{~b}(\mathrm{i}) * \mathrm{Wq})))) /(8 * \mathrm{pi})$;

$\mathrm{S} 2223$

$\left(\left(\mathrm{L}(1,4) *\left(\operatorname{sum}(\mathrm{g} 2121 \mathrm{a}(\mathrm{i}) . * \mathrm{Wq})+\operatorname{sum}(\mathrm{g} 2121 \mathrm{~b}(\mathrm{i}) . * \mathrm{Wq})+\operatorname{sum}\left(\mathrm{g} 2121 \mathrm{a}(\mathrm{i}) .{ }^{*} \mathrm{Wq}\right)+\operatorname{sum}(\mathrm{g} 2\right.\right.\right.$ $121 \mathrm{~b}(\mathrm{i}) * \mathrm{Wq})))$

$\left(\mathrm{L}(6,4) *\left(\operatorname{sum}\left(\mathrm{g} 2122 \mathrm{a}(\mathrm{i}) .{ }^{*} \mathrm{Wq}\right)+\operatorname{sum}\left(\mathrm{g} 2122 \mathrm{~b}(\mathrm{i}) .{ }^{*} \mathrm{Wq}\right)+\operatorname{sum}\left(\mathrm{g} 2122 \mathrm{a}(\mathrm{i}) .{ }^{*} \mathrm{Wq}\right)+\operatorname{sum}(\mathrm{g} 21\right.\right.$ $22 \mathrm{~b}(\mathrm{i}) . * \mathrm{Wq})))$

$\left(\mathrm{L}(8,4) *\left(\operatorname{sum}\left(\mathrm{g} 2123 \mathrm{a}(\mathrm{i}) .{ }^{*} \mathrm{Wq}\right)+\operatorname{sum}\left(\mathrm{g} 2123 \mathrm{~b}(\mathrm{i}) .{ }^{*} \mathrm{Wq}\right)+\operatorname{sum}\left(\mathrm{g} 2123 \mathrm{a}(\mathrm{i}) .{ }^{*} \mathrm{Wq}\right)+\operatorname{sum}(\mathrm{g} 21\right.\right.$

$23 \mathrm{~b}(\mathrm{i}) \cdot * \mathrm{Wq})))$

$\left(\mathrm{L}(9,4) *\left(\operatorname{sum}(\mathrm{g} 2221 \mathrm{a}(\mathrm{i}) . * \mathrm{Wq})+\operatorname{sum}\left(\mathrm{g} 2221 \mathrm{~b}(\mathrm{i}) \cdot{ }^{*} \mathrm{Wq}\right)+\operatorname{sum}\left(\mathrm{g} 2221 \mathrm{a}(\mathrm{i}) \cdot{ }^{*} \mathrm{Wq}\right)+\operatorname{sum}(\mathrm{g} 22\right.\right.$

$21 \mathrm{~b}(\mathrm{i}) . * \mathrm{Wq})))$ 
$\left(\mathrm{L}(2,4) *\left(\operatorname{sum}(\mathrm{g} 2222 \mathrm{a}(\mathrm{i}) \cdot * \mathrm{Wq})+\operatorname{sum}(\mathrm{g} 2222 \mathrm{~b}(\mathrm{i}) \cdot * \mathrm{Wq})+\operatorname{sum}\left(\mathrm{g} 2222 \mathrm{a}(\mathrm{i}) \cdot{ }^{*} \mathrm{Wq}\right)+\operatorname{sum}(\mathrm{g} 22\right.\right.$ $22 \mathrm{~b}(\mathrm{i}) . * \mathrm{Wq})))$ $(\mathrm{L}(4,4) *(\operatorname{sum}(\mathrm{g} 2223 \mathrm{a}(\mathrm{i}) . * \mathrm{Wq})+\operatorname{sum}(\mathrm{g} 2223 \mathrm{~b}(\mathrm{i}) . * \mathrm{Wq})+\operatorname{sum}(\mathrm{g} 2223 \mathrm{a}(\mathrm{i}) . * \mathrm{Wq})+\operatorname{sum}(\mathrm{g} 22$ $23 \mathrm{~b}(\mathrm{i}) . * \mathrm{Wq})))$ $\left(\mathrm{L}(5,4) *\left(\operatorname{sum}\left(\mathrm{g} 2321 \mathrm{a}(\mathrm{i}) .{ }^{*} \mathrm{Wq}\right)+\operatorname{sum}\left(\mathrm{g} 2321 \mathrm{~b}(\mathrm{i}) .{ }^{*} \mathrm{Wq}\right)+\operatorname{sum}\left(\mathrm{g} 2321 \mathrm{a}(\mathrm{i}) .{ }^{*} \mathrm{Wq}\right)+\operatorname{sum}(\mathrm{g} 23\right.\right.$ $21 \mathrm{~b}(\mathrm{i}) . * \mathrm{Wq})))$ $\left(\mathrm{L}(7,4) *\left(\operatorname{sum}\left(\mathrm{g} 2322 \mathrm{a}(\mathrm{i}) .{ }^{*} \mathrm{Wq}\right)+\operatorname{sum}\left(\mathrm{g} 2322 \mathrm{~b}(\mathrm{i}) .{ }^{*} \mathrm{Wq}\right)+\operatorname{sum}(\mathrm{g} 2322 \mathrm{a}(\mathrm{i}) . * \mathrm{Wq})+\operatorname{sum}(\mathrm{g} 23\right.\right.$ $22 \mathrm{~b}(\mathrm{i}) . * \mathrm{Wq})))$ $\left(\mathrm{L}(3,4) *\left(\operatorname{sum}\left(\mathrm{g} 2323 \mathrm{a}(\mathrm{i}) .{ }^{*} \mathrm{Wq}\right)+\operatorname{sum}\left(\mathrm{g} 2323 \mathrm{~b}(\mathrm{i}) .{ }^{*} \mathrm{Wq}\right)+\operatorname{sum}\left(\mathrm{g} 2323 \mathrm{a}(\mathrm{i}) .{ }^{*} \mathrm{Wq}\right)+\operatorname{sum}(\mathrm{g} 23\right.\right.$ $\left.\left.\left.\left.23 \mathrm{~b}(\mathrm{i}) \cdot{ }^{*} \mathrm{Wq}\right)\right)\right)\right) /(8 * \mathrm{pi})$;

$\mathrm{S} 2231$

$\left(\left(\mathrm{L}(1,5) *\left(\operatorname{sum}\left(\mathrm{g} 2121 \mathrm{a}(\mathrm{i}) .{ }^{*} \mathrm{Wq}\right)+\operatorname{sum}\left(\mathrm{g} 2121 \mathrm{~b}(\mathrm{i}) .{ }^{*} \mathrm{Wq}\right)+\operatorname{sum}\left(\mathrm{g} 2121 \mathrm{a}(\mathrm{i}) .{ }^{*} \mathrm{Wq}\right)+\operatorname{sum}(\mathrm{g} 2\right.\right.\right.$ $\left.\left.\left.121 \mathrm{~b}(\mathrm{i}) \cdot{ }^{*} \mathrm{Wq}\right)\right)\right)$

$\left(\mathrm{L}(6,5) *\left(\operatorname{sum}\left(\mathrm{g} 2122 \mathrm{a}(\mathrm{i}) .{ }^{*} \mathrm{Wq}\right)+\operatorname{sum}\left(\mathrm{g} 2122 \mathrm{~b}(\mathrm{i}) .{ }^{*} \mathrm{Wq}\right)+\operatorname{sum}\left(\mathrm{g} 2122 \mathrm{a}(\mathrm{i}) .{ }^{*} \mathrm{Wq}\right)+\operatorname{sum}(\mathrm{g} 21\right.\right.$ $22 \mathrm{~b}(\mathrm{i}) . * \mathrm{Wq}))$ )

$\left(\mathrm{L}(8,5) *\left(\operatorname{sum}(\mathrm{g} 2123 \mathrm{a}(\mathrm{i}) . * \mathrm{Wq})+\operatorname{sum}\left(\mathrm{g} 2123 \mathrm{~b}(\mathrm{i}) \cdot{ }^{*} \mathrm{Wq}\right)+\operatorname{sum}\left(\mathrm{g} 2123 \mathrm{a}(\mathrm{i}) .{ }^{*} \mathrm{Wq}\right)+\operatorname{sum}(\mathrm{g} 21\right.\right.$ $23 \mathrm{~b}(\mathrm{i}) \cdot * \mathrm{Wq})))$ $\left(\mathrm{L}(9,5) *\left(\operatorname{sum}\left(\mathrm{g} 2221 \mathrm{a}(\mathrm{i}) .{ }^{*} \mathrm{Wq}\right)+\operatorname{sum}\left(\mathrm{g} 2221 \mathrm{~b}(\mathrm{i}) .{ }^{*} \mathrm{Wq}\right)+\operatorname{sum}\left(\mathrm{g} 2221 \mathrm{a}(\mathrm{i}) \cdot{ }^{*} \mathrm{Wq}\right)+\operatorname{sum}(\mathrm{g} 22\right.\right.$ $21 \mathrm{~b}(\mathrm{i}) . * \mathrm{Wq}))$

$\left(\mathrm{L}(2,5) *\left(\operatorname{sum}\left(\mathrm{g} 2222 \mathrm{a}(\mathrm{i}) .{ }^{*} \mathrm{Wq}\right)+\operatorname{sum}\left(\mathrm{g} 2222 \mathrm{~b}(\mathrm{i}) \cdot{ }^{*} \mathrm{Wq}\right)+\operatorname{sum}\left(\mathrm{g} 2222 \mathrm{a}(\mathrm{i}) .{ }^{*} \mathrm{Wq}\right)+\operatorname{sum}(\mathrm{g} 22\right.\right.$ $22 \mathrm{~b}(\mathrm{i}) . * \mathrm{Wq})))$

$\left(\mathrm{L}(4,5) *\left(\operatorname{sum}(\mathrm{g} 2223 \mathrm{a}(\mathrm{i}) . * \mathrm{Wq})+\operatorname{sum}\left(\mathrm{g} 2223 \mathrm{~b}(\mathrm{i}) \cdot{ }^{*} \mathrm{Wq}\right)+\operatorname{sum}\left(\mathrm{g} 2223 \mathrm{a}(\mathrm{i}) \cdot{ }^{*} \mathrm{Wq}\right)+\operatorname{sum}(\mathrm{g} 22\right.\right.$ $23 \mathrm{~b}(\mathrm{i}) . * \mathrm{Wq})))$ $\left(\mathrm{L}(5,5) *\left(\operatorname{sum}\left(\mathrm{g} 2321 \mathrm{a}(\mathrm{i}) .{ }^{*} \mathrm{Wq}\right)+\operatorname{sum}\left(\mathrm{g} 2321 \mathrm{~b}(\mathrm{i}) .{ }^{*} \mathrm{Wq}\right)+\operatorname{sum}\left(\mathrm{g} 2321 \mathrm{a}(\mathrm{i}) .{ }^{*} \mathrm{Wq}\right)+\operatorname{sum}(\mathrm{g} 23\right.\right.$ $21 \mathrm{~b}(\mathrm{i}) . * \mathrm{Wq})))$

$\left(\mathrm{L}(7,5) *\left(\operatorname{sum}\left(\mathrm{g} 2322 \mathrm{a}(\mathrm{i}) .{ }^{*} \mathrm{Wq}\right)+\operatorname{sum}\left(\mathrm{g} 2322 \mathrm{~b}(\mathrm{i}) .{ }^{*} \mathrm{Wq}\right)+\operatorname{sum}\left(\mathrm{g} 2322 \mathrm{a}(\mathrm{i}) \cdot{ }^{*} \mathrm{Wq}\right)+\operatorname{sum}(\mathrm{g} 23\right.\right.$ $22 \mathrm{~b}(\mathrm{i}) . * \mathrm{Wq}))$

$\left(\mathrm{L}(3,5) *\left(\operatorname{sum}\left(\mathrm{g} 2323 \mathrm{a}(\mathrm{i}) .{ }^{*} \mathrm{Wq}\right)+\operatorname{sum}(\mathrm{g} 2323 \mathrm{~b}(\mathrm{i}) . * \mathrm{Wq})+\operatorname{sum}\left(\mathrm{g} 2323 \mathrm{a}(\mathrm{i}) .{ }^{*} \mathrm{Wq}\right)+\operatorname{sum}(\mathrm{g} 23\right.\right.$ $23 \mathrm{~b}(\mathrm{i}) . * \mathrm{Wq})))) /(8 * \mathrm{pi})$;

$\mathrm{S} 2212$

$\left(\left(\mathrm{L}(1,6) *\left(\operatorname{sum}\left(\mathrm{g} 2121 \mathrm{a}(\mathrm{i}) .{ }^{*} \mathrm{Wq}\right)+\operatorname{sum}\left(\mathrm{g} 2121 \mathrm{~b}(\mathrm{i}) .{ }^{*} \mathrm{Wq}\right)+\operatorname{sum}\left(\mathrm{g} 2121 \mathrm{a}(\mathrm{i}) .{ }^{*} \mathrm{Wq}\right)+\operatorname{sum}(\mathrm{g} 2\right.\right.\right.$ $\left.\left.121 \mathrm{~b}(\mathrm{i}) \cdot{ }^{*} \mathrm{Wq}\right)\right)$

$\left(\mathrm{L}(6,6) *\left(\operatorname{sum}\left(\mathrm{g} 2122 \mathrm{a}(\mathrm{i}) .{ }^{*} \mathrm{Wq}\right)+\operatorname{sum}\left(\mathrm{g} 2122 \mathrm{~b}(\mathrm{i}) \cdot{ }^{*} \mathrm{Wq}\right)+\operatorname{sum}(\mathrm{g} 2122 \mathrm{a}(\mathrm{i}) . * \mathrm{Wq})+\operatorname{sum}(\mathrm{g} 21\right.\right.$ $22 \mathrm{~b}(\mathrm{i}) . * \mathrm{Wq})))$

$\left(\mathrm{L}(8,6) *\left(\operatorname{sum}\left(\mathrm{g} 2123 \mathrm{a}(\mathrm{i}) .{ }^{*} \mathrm{Wq}\right)+\operatorname{sum}\left(\mathrm{g} 2123 \mathrm{~b}(\mathrm{i}) .{ }^{*} \mathrm{Wq}\right)+\operatorname{sum}\left(\mathrm{g} 2123 \mathrm{a}(\mathrm{i}) .{ }^{*} \mathrm{Wq}\right)+\operatorname{sum}(\mathrm{g} 21\right.\right.$ $23 \mathrm{~b}(\mathrm{i}) \cdot * \mathrm{Wq}))$

$\left(\mathrm{L}(9,6) *\left(\operatorname{sum}\left(\mathrm{g} 2221 \mathrm{a}(\mathrm{i}) .{ }^{*} \mathrm{Wq}\right)+\operatorname{sum}\left(\mathrm{g} 2221 \mathrm{~b}(\mathrm{i}) .{ }^{*} \mathrm{Wq}\right)+\operatorname{sum}(\mathrm{g} 2221 \mathrm{a}(\mathrm{i}) . * \mathrm{Wq})+\operatorname{sum}(\mathrm{g} 22\right.\right.$ $21 \mathrm{~b}(\mathrm{i}) . * \mathrm{Wq})))$

$\left(\mathrm{L}(2,6) *\left(\operatorname{sum}\left(\mathrm{g} 2222 \mathrm{a}(\mathrm{i}) .{ }^{*} \mathrm{Wq}\right)+\operatorname{sum}\left(\mathrm{g} 2222 \mathrm{~b}(\mathrm{i}) .{ }^{*} \mathrm{Wq}\right)+\operatorname{sum}\left(\mathrm{g} 2222 \mathrm{a}(\mathrm{i}) .{ }^{*} \mathrm{Wq}\right)+\operatorname{sum}(\mathrm{g} 22\right.\right.$ $22 \mathrm{~b}(\mathrm{i}) . * \mathrm{Wq})))$ $\left(\mathrm{L}(4,6) *\left(\operatorname{sum}\left(\mathrm{g} 2223 \mathrm{a}(\mathrm{i}) .{ }^{*} \mathrm{Wq}\right)+\operatorname{sum}\left(\mathrm{g} 2223 \mathrm{~b}(\mathrm{i}) .{ }^{*} \mathrm{Wq}\right)+\operatorname{sum}\left(\mathrm{g} 2223 \mathrm{a}(\mathrm{i}) .{ }^{*} \mathrm{Wq}\right)+\operatorname{sum}(\mathrm{g} 22\right.\right.$ $23 \mathrm{~b}(\mathrm{i}) . * \mathrm{Wq})))$ $\left(\mathrm{L}(5,6) *\left(\operatorname{sum}\left(\mathrm{g} 2321 \mathrm{a}(\mathrm{i}) .{ }^{*} \mathrm{Wq}\right)+\operatorname{sum}\left(\mathrm{g} 2321 \mathrm{~b}(\mathrm{i}) .{ }^{*} \mathrm{Wq}\right)+\operatorname{sum}\left(\mathrm{g} 2321 \mathrm{a}(\mathrm{i}) .{ }^{*} \mathrm{Wq}\right)+\operatorname{sum}(\mathrm{g} 23\right.\right.$ 
$21 \mathrm{~b}(\mathrm{i}) . * \mathrm{Wq})))$

$\left(\mathrm{L}(7,6) *\left(\operatorname{sum}\left(\mathrm{g} 2322 \mathrm{a}(\mathrm{i}) .{ }^{*} \mathrm{Wq}\right)+\operatorname{sum}(\mathrm{g} 2322 \mathrm{~b}(\mathrm{i}) . * \mathrm{Wq})+\operatorname{sum}\left(\mathrm{g} 2322 \mathrm{a}(\mathrm{i}) .{ }^{*} \mathrm{Wq}\right)+\operatorname{sum}(\mathrm{g} 23\right.\right.$

$22 \mathrm{~b}(\mathrm{i}) . * \mathrm{Wq})))$

$\left(\mathrm{L}(3,6) *\left(\operatorname{sum}(\mathrm{g} 2323 \mathrm{a}(\mathrm{i}) . * \mathrm{Wq})+\operatorname{sum}\left(\mathrm{g} 2323 \mathrm{~b}(\mathrm{i}) \cdot{ }^{*} \mathrm{Wq}\right)+\operatorname{sum}\left(\mathrm{g} 2323 \mathrm{a}(\mathrm{i}) \cdot{ }^{*} \mathrm{Wq}\right)+\operatorname{sum}(\mathrm{g} 23\right.\right.$

$\left.\left.\left.\left.23 \mathrm{~b}(\mathrm{i}) \cdot{ }^{*} \mathrm{Wq}\right)\right)\right)\right) /(8 * \mathrm{pi})$;

$\mathrm{S} 2232$

$\left(\left(\mathrm{L}(1,7) *\left(\operatorname{sum}\left(\mathrm{g} 2121 \mathrm{a}(\mathrm{i}) .{ }^{*} \mathrm{Wq}\right)+\operatorname{sum}\left(\mathrm{g} 2121 \mathrm{~b}(\mathrm{i}) .{ }^{*} \mathrm{Wq}\right)+\operatorname{sum}\left(\mathrm{g} 2121 \mathrm{a}(\mathrm{i}) .{ }^{*} \mathrm{Wq}\right)+\operatorname{sum}(\mathrm{g} 2\right.\right.\right.$ $\left.\left.\left.121 \mathrm{~b}(\mathrm{i}) \cdot{ }^{*} \mathrm{Wq}\right)\right)\right)$

$\left(\mathrm{L}(6,7) *\left(\operatorname{sum}\left(\mathrm{g} 2122 \mathrm{a}(\mathrm{i}) .{ }^{*} \mathrm{Wq}\right)+\operatorname{sum}\left(\mathrm{g} 2122 \mathrm{~b}(\mathrm{i}) .{ }^{*} \mathrm{Wq}\right)+\operatorname{sum}(\mathrm{g} 2122 \mathrm{a}(\mathrm{i}) . * \mathrm{Wq})+\operatorname{sum}(\mathrm{g} 21\right.\right.$

$22 \mathrm{~b}(\mathrm{i}) . * \mathrm{Wq})))$

$\left(\mathrm{L}(8,7) *\left(\operatorname{sum}\left(\mathrm{g} 2123 \mathrm{a}(\mathrm{i}) .{ }^{*} \mathrm{Wq}\right)+\operatorname{sum}\left(\mathrm{g} 2123 \mathrm{~b}(\mathrm{i}) .{ }^{*} \mathrm{Wq}\right)+\operatorname{sum}\left(\mathrm{g} 2123 \mathrm{a}(\mathrm{i}) .{ }^{*} \mathrm{Wq}\right)+\operatorname{sum}(\mathrm{g} 21\right.\right.$

$23 \mathrm{~b}(\mathrm{i}) . * \mathrm{Wq})))$

$\left(\mathrm{L}(9,7) *\left(\operatorname{sum}\left(\mathrm{g} 2221 \mathrm{a}(\mathrm{i}) .{ }^{*} \mathrm{Wq}\right)+\operatorname{sum}\left(\mathrm{g} 2221 \mathrm{~b}(\mathrm{i}) .{ }^{*} \mathrm{Wq}\right)+\operatorname{sum}\left(\mathrm{g} 2221 \mathrm{a}(\mathrm{i}) .{ }^{*} \mathrm{Wq}\right)+\operatorname{sum}(\mathrm{g} 22\right.\right.$

$21 \mathrm{~b}(\mathrm{i}) \cdot * \mathrm{Wq}))$

$\left(\mathrm{L}(2,7) *\left(\operatorname{sum}\left(\mathrm{g} 2222 \mathrm{a}(\mathrm{i}) .{ }^{*} \mathrm{Wq}\right)+\operatorname{sum}\left(\mathrm{g} 2222 \mathrm{~b}(\mathrm{i}) \cdot{ }^{*} \mathrm{Wq}\right)+\operatorname{sum}(\mathrm{g} 2222 \mathrm{a}(\mathrm{i}) . * \mathrm{Wq})+\operatorname{sum}(\mathrm{g} 22\right.\right.$

$22 \mathrm{~b}(\mathrm{i}) . * \mathrm{Wq})))$

$\left(\mathrm{L}(4,7) *\left(\operatorname{sum}\left(\mathrm{g} 2223 \mathrm{a}(\mathrm{i}) .{ }^{*} \mathrm{Wq}\right)+\operatorname{sum}\left(\mathrm{g} 2223 \mathrm{~b}(\mathrm{i}) \cdot{ }^{*} \mathrm{Wq}\right)+\operatorname{sum}\left(\mathrm{g} 2223 \mathrm{a}(\mathrm{i}) .{ }^{*} \mathrm{Wq}\right)+\operatorname{sum}(\mathrm{g} 22\right.\right.$

$23 \mathrm{~b}(\mathrm{i}) \cdot * \mathrm{Wq}))$

$\left(\mathrm{L}(5,7) *\left(\operatorname{sum}\left(\mathrm{g} 2321 \mathrm{a}(\mathrm{i}) .{ }^{*} \mathrm{Wq}\right)+\operatorname{sum}\left(\mathrm{g} 2321 \mathrm{~b}(\mathrm{i}) .{ }^{*} \mathrm{Wq}\right)+\operatorname{sum}\left(\mathrm{g} 2321 \mathrm{a}(\mathrm{i}) .{ }^{*} \mathrm{Wq}\right)+\operatorname{sum}(\mathrm{g} 23\right.\right.$

$21 \mathrm{~b}(\mathrm{i}) . * \mathrm{Wq})))$

$\left(\mathrm{L}(7,7) *\left(\operatorname{sum}\left(\mathrm{g} 2322 \mathrm{a}(\mathrm{i}) .{ }^{*} \mathrm{Wq}\right)+\operatorname{sum}\left(\mathrm{g} 2322 \mathrm{~b}(\mathrm{i}) \cdot{ }^{*} \mathrm{Wq}\right)+\operatorname{sum}\left(\mathrm{g} 2322 \mathrm{a}(\mathrm{i}) .{ }^{*} \mathrm{Wq}\right)+\operatorname{sum}(\mathrm{g} 23\right.\right.$

$22 \mathrm{~b}(\mathrm{i}) . * \mathrm{Wq})))$

$\left(\mathrm{L}(3,7) *\left(\operatorname{sum}\left(\mathrm{g} 2323 \mathrm{a}(\mathrm{i}) .{ }^{*} \mathrm{Wq}\right)+\operatorname{sum}\left(\mathrm{g} 2323 \mathrm{~b}(\mathrm{i}) .{ }^{*} \mathrm{Wq}\right)+\operatorname{sum}\left(\mathrm{g} 2323 \mathrm{a}(\mathrm{i}) .{ }^{*} \mathrm{Wq}\right)+\operatorname{sum}(\mathrm{g} 23\right.\right.$

$23 \mathrm{~b}(\mathrm{i}) * \mathrm{Wq})))) /(8 * \mathrm{pi})$

$\mathrm{S} 2213$

$\left(\left(\mathrm{L}(1,8) *\left(\operatorname{sum}\left(\mathrm{g} 2121 \mathrm{a}(\mathrm{i}) .{ }^{*} \mathrm{Wq}\right)+\operatorname{sum}\left(\mathrm{g} 2121 \mathrm{~b}(\mathrm{i}) .{ }^{*} \mathrm{Wq}\right)+\operatorname{sum}\left(\mathrm{g} 2121 \mathrm{a}(\mathrm{i}) .{ }^{*} \mathrm{Wq}\right)+\operatorname{sum}(\mathrm{g} 2\right.\right.\right.$ $\left.\left.\left.121 \mathrm{~b}(\mathrm{i}) \cdot{ }^{*} \mathrm{Wq}\right)\right)\right)$

$\left(\mathrm{L}(6,8) *\left(\operatorname{sum}\left(\mathrm{g} 2122 \mathrm{a}(\mathrm{i}) .{ }^{*} \mathrm{Wq}\right)+\operatorname{sum}\left(\mathrm{g} 2122 \mathrm{~b}(\mathrm{i}) .{ }^{*} \mathrm{Wq}\right)+\operatorname{sum}\left(\mathrm{g} 2122 \mathrm{a}(\mathrm{i}) .{ }^{*} \mathrm{Wq}\right)+\operatorname{sum}(\mathrm{g} 21\right.\right.$

$22 \mathrm{~b}(\mathrm{i}) . * \mathrm{Wq})))$

$\left(\mathrm{L}(8,8) *\left(\operatorname{sum}\left(\mathrm{g} 2123 \mathrm{a}(\mathrm{i}) .{ }^{*} \mathrm{Wq}\right)+\operatorname{sum}\left(\mathrm{g} 2123 \mathrm{~b}(\mathrm{i}) .{ }^{*} \mathrm{Wq}\right)+\operatorname{sum}(\mathrm{g} 2123 \mathrm{a}(\mathrm{i}) . * \mathrm{Wq})+\operatorname{sum}(\mathrm{g} 21\right.\right.$

$23 \mathrm{~b}(\mathrm{i}) \cdot * \mathrm{Wq}))$

$\left(\mathrm{L}(9,8) *\left(\operatorname{sum}\left(\mathrm{g} 2221 \mathrm{a}(\mathrm{i}) .{ }^{*} \mathrm{Wq}\right)+\operatorname{sum}\left(\mathrm{g} 2221 \mathrm{~b}(\mathrm{i}) .{ }^{*} \mathrm{Wq}\right)+\operatorname{sum}\left(\mathrm{g} 2221 \mathrm{a}(\mathrm{i}) .{ }^{*} \mathrm{Wq}\right)+\operatorname{sum}(\mathrm{g} 22\right.\right.$

$21 \mathrm{~b}(\mathrm{i}) . * \mathrm{Wq})))$

$\left(\mathrm{L}(2,8) *\left(\operatorname{sum}\left(\mathrm{g} 2222 \mathrm{a}(\mathrm{i}) .{ }^{*} \mathrm{Wq}\right)+\operatorname{sum}\left(\mathrm{g} 2222 \mathrm{~b}(\mathrm{i}) \cdot{ }^{*} \mathrm{Wq}\right)+\operatorname{sum}\left(\mathrm{g} 2222 \mathrm{a}(\mathrm{i}) \cdot{ }^{*} \mathrm{Wq}\right)+\operatorname{sum}(\mathrm{g} 22\right.\right.$

$22 \mathrm{~b}(\mathrm{i}) . * \mathrm{Wq})))$

$\left(\mathrm{L}(4,8) *\left(\operatorname{sum}\left(\mathrm{g} 2223 \mathrm{a}(\mathrm{i}) .{ }^{*} \mathrm{Wq}\right)+\operatorname{sum}\left(\mathrm{g} 2223 \mathrm{~b}(\mathrm{i}) \cdot{ }^{*} \mathrm{Wq}\right)+\operatorname{sum}(\mathrm{g} 2223 \mathrm{a}(\mathrm{i}) . * \mathrm{Wq})+\operatorname{sum}(\mathrm{g} 22\right.\right.$

$23 \mathrm{~b}(\mathrm{i}) . * \mathrm{Wq})))$

$\left(\mathrm{L}(5,8) *\left(\operatorname{sum}\left(\mathrm{g} 2321 \mathrm{a}(\mathrm{i}) .{ }^{*} \mathrm{Wq}\right)+\operatorname{sum}\left(\mathrm{g} 2321 \mathrm{~b}(\mathrm{i}) .{ }^{*} \mathrm{Wq}\right)+\operatorname{sum}\left(\mathrm{g} 2321 \mathrm{a}(\mathrm{i}) .{ }^{*} \mathrm{Wq}\right)+\operatorname{sum}(\mathrm{g} 23\right.\right.$

$21 \mathrm{~b}(\mathrm{i}) . * \mathrm{Wq})))$

$\left(\mathrm{L}(7,8) *\left(\operatorname{sum}\left(\mathrm{g} 2322 \mathrm{a}(\mathrm{i}) .{ }^{*} \mathrm{Wq}\right)+\operatorname{sum}\left(\mathrm{g} 2322 \mathrm{~b}(\mathrm{i}) \cdot{ }^{*} \mathrm{Wq}\right)+\operatorname{sum}(\mathrm{g} 2322 \mathrm{a}(\mathrm{i}) . * \mathrm{Wq})+\operatorname{sum}(\mathrm{g} 23\right.\right.$

$22 \mathrm{~b}(\mathrm{i}) . * \mathrm{Wq})))$

$\left(\mathrm{L}(3,8) *\left(\operatorname{sum}(\mathrm{g} 2323 \mathrm{a}(\mathrm{i}) . * \mathrm{Wq})+\operatorname{sum}\left(\mathrm{g} 2323 \mathrm{~b}(\mathrm{i}) \cdot{ }^{*} \mathrm{Wq}\right)+\operatorname{sum}\left(\mathrm{g} 2323 \mathrm{a}(\mathrm{i}) \cdot{ }^{*} \mathrm{Wq}\right)+\operatorname{sum}(\mathrm{g} 23\right.\right.$

$\left.\left.\left.\left.23 \mathrm{~b}(\mathrm{i}){ }^{*} \mathrm{Wq}\right)\right)\right)\right) /(8 * \mathrm{pi})$; 
S2221

$\left(\left(\mathrm{L}(1,9) *\left(\operatorname{sum}\left(\mathrm{g} 2121 \mathrm{a}(\mathrm{i}) .{ }^{*} \mathrm{Wq}\right)+\operatorname{sum}\left(\mathrm{g} 2121 \mathrm{~b}(\mathrm{i}) .{ }^{*} \mathrm{Wq}\right)+\operatorname{sum}\left(\mathrm{g} 2121 \mathrm{a}(\mathrm{i}) .{ }^{*} \mathrm{Wq}\right)+\operatorname{sum}(\mathrm{g} 2\right.\right.\right.$ $\left.\left.\left.121 \mathrm{~b}(\mathrm{i}) \cdot{ }^{*} \mathrm{Wq}\right)\right)\right)$

$\left(\mathrm{L}(6,9) *\left(\operatorname{sum}(\mathrm{g} 2122 \mathrm{a}(\mathrm{i}) . * \mathrm{Wq})+\operatorname{sum}\left(\mathrm{g} 2122 \mathrm{~b}(\mathrm{i}) \cdot{ }^{*} \mathrm{Wq}\right)+\operatorname{sum}\left(\mathrm{g} 2122 \mathrm{a}(\mathrm{i}) \cdot{ }^{*} \mathrm{Wq}\right)+\operatorname{sum}(\mathrm{g} 21\right.\right.$ $\left.\left.\left.22 \mathrm{~b}(\mathrm{i}) \cdot{ }^{*} \mathrm{Wq}\right)\right)\right)$

$\left(\mathrm{L}(8,9) *\left(\operatorname{sum}\left(\mathrm{g} 2123 \mathrm{a}(\mathrm{i}) .{ }^{*} \mathrm{Wq}\right)+\operatorname{sum}\left(\mathrm{g} 2123 \mathrm{~b}(\mathrm{i}) .{ }^{*} \mathrm{Wq}\right)+\operatorname{sum}\left(\mathrm{g} 2123 \mathrm{a}(\mathrm{i}) .{ }^{*} \mathrm{Wq}\right)+\operatorname{sum}(\mathrm{g} 21\right.\right.$

$\left.\left.\left.23 \mathrm{~b}(\mathrm{i}) \cdot{ }^{*} \mathrm{Wq}\right)\right)\right)$

$\left(\mathrm{L}(9,9) *\left(\operatorname{sum}(\mathrm{g} 2221 \mathrm{a}(\mathrm{i}) . * \mathrm{Wq})+\operatorname{sum}\left(\mathrm{g} 2221 \mathrm{~b}(\mathrm{i}) \cdot{ }^{*} \mathrm{Wq}\right)+\operatorname{sum}\left(\mathrm{g} 2221 \mathrm{a}(\mathrm{i}) .{ }^{*} \mathrm{Wq}\right)+\operatorname{sum}(\mathrm{g} 22\right.\right.$

$21 \mathrm{~b}(\mathrm{i}) . * \mathrm{Wq}))$

$\left(\mathrm{L}(2,9) *\left(\operatorname{sum}\left(\mathrm{g} 2222 \mathrm{a}(\mathrm{i}) .{ }^{*} \mathrm{Wq}\right)+\operatorname{sum}\left(\mathrm{g} 2222 \mathrm{~b}(\mathrm{i}) \cdot{ }^{*} \mathrm{Wq}\right)+\operatorname{sum}(\mathrm{g} 2222 \mathrm{a}(\mathrm{i}) . * \mathrm{Wq})+\operatorname{sum}(\mathrm{g} 22\right.\right.$

$22 \mathrm{~b}(\mathrm{i}) . * \mathrm{Wq})))$

$\left(\mathrm{L}(4,9) *\left(\operatorname{sum}\left(\mathrm{g} 2223 \mathrm{a}(\mathrm{i}) .{ }^{*} \mathrm{Wq}\right)+\operatorname{sum}\left(\mathrm{g} 2223 \mathrm{~b}(\mathrm{i}) .{ }^{*} \mathrm{Wq}\right)+\operatorname{sum}(\mathrm{g} 2223 \mathrm{a}(\mathrm{i}) . * \mathrm{Wq})+\operatorname{sum}(\mathrm{g} 22\right.\right.$

$23 \mathrm{~b}(\mathrm{i}) . * \mathrm{Wq})))$

$\left(\mathrm{L}(5,9) *\left(\operatorname{sum}\left(\mathrm{g} 2321 \mathrm{a}(\mathrm{i}) .{ }^{*} \mathrm{Wq}\right)+\operatorname{sum}\left(\mathrm{g} 2321 \mathrm{~b}(\mathrm{i}) .{ }^{*} \mathrm{Wq}\right)+\operatorname{sum}\left(\mathrm{g} 2321 \mathrm{a}(\mathrm{i}) .{ }^{*} \mathrm{Wq}\right)+\operatorname{sum}(\mathrm{g} 23\right.\right.$

$21 \mathrm{~b}(\mathrm{i}) . * \mathrm{Wq})))$

$\left(\mathrm{L}(7,9) *\left(\operatorname{sum}(\mathrm{g} 2322 \mathrm{a}(\mathrm{i}) . * \mathrm{Wq})+\operatorname{sum}\left(\mathrm{g} 2322 \mathrm{~b}(\mathrm{i}) \cdot{ }^{*} \mathrm{Wq}\right)+\operatorname{sum}(\mathrm{g} 2322 \mathrm{a}(\mathrm{i}) . * \mathrm{Wq})+\operatorname{sum}(\mathrm{g} 23\right.\right.$

$22 \mathrm{~b}(\mathrm{i}) . * \mathrm{Wq}))$

$\left(\mathrm{L}(3,9) *\left(\operatorname{sum}\left(\mathrm{g} 2323 \mathrm{a}(\mathrm{i}) .{ }^{*} \mathrm{Wq}\right)+\operatorname{sum}\left(\mathrm{g} 2323 \mathrm{~b}(\mathrm{i}) .{ }^{*} \mathrm{Wq}\right)+\operatorname{sum}(\mathrm{g} 2323 \mathrm{a}(\mathrm{i}) . * \mathrm{Wq})+\operatorname{sum}(\mathrm{g} 23\right.\right.$

$23 \mathrm{~b}(\mathrm{i}) * \mathrm{Wq})))) /(8 * \mathrm{pi})$;

S3311

$\left(\left(\mathrm{L}(1,1) *\left(\operatorname{sum}\left(\mathrm{g} 3131 \mathrm{a}(\mathrm{i}) .{ }^{*} \mathrm{Wq}\right)+\operatorname{sum}\left(\mathrm{g} 3131 \mathrm{~b}(\mathrm{i}) .{ }^{*} \mathrm{Wq}\right)+\operatorname{sum}\left(\mathrm{g} 3131 \mathrm{a}(\mathrm{i}) .{ }^{*} \mathrm{Wq}\right)+\operatorname{sum}(\mathrm{g} 3\right.\right.\right.$ $\left.\left.\left.131 \mathrm{~b}(\mathrm{i}) *{ }^{*} \mathrm{Wq}\right)\right)\right)$

$\left(\mathrm{L}(6,1) *\left(\operatorname{sum}\left(\mathrm{g} 3132 \mathrm{a}(\mathrm{i}) .{ }^{*} \mathrm{Wq}\right)+\operatorname{sum}\left(\mathrm{g} 3132 \mathrm{~b}(\mathrm{i}) .{ }^{*} \mathrm{Wq}\right)+\operatorname{sum}(\mathrm{g} 3132 \mathrm{a}(\mathrm{i}) . * \mathrm{Wq})+\operatorname{sum}(\mathrm{g} 31\right.\right.$ $32 \mathrm{~b}(\mathrm{i}) . * \mathrm{Wq})))$

$\left(\mathrm{L}(8,1) *\left(\operatorname{sum}\left(\mathrm{g} 3133 \mathrm{a}(\mathrm{i}) .{ }^{*} \mathrm{Wq}\right)+\operatorname{sum}\left(\mathrm{g} 3133 \mathrm{~b}(\mathrm{i}) .{ }^{*} \mathrm{Wq}\right)+\operatorname{sum}(\mathrm{g} 3133 \mathrm{a}(\mathrm{i}) . * \mathrm{Wq})+\operatorname{sum}(\mathrm{g} 31\right.\right.$

$33 \mathrm{~b}(\mathrm{i}) . * \mathrm{Wq}))$

$\left(\mathrm{L}(9,1) *\left(\operatorname{sum}\left(\mathrm{g} 3231 \mathrm{a}(\mathrm{i}) .{ }^{*} \mathrm{Wq}\right)+\operatorname{sum}\left(\mathrm{g} 3231 \mathrm{~b}(\mathrm{i}) .{ }^{*} \mathrm{Wq}\right)+\operatorname{sum}\left(\mathrm{g} 3231 \mathrm{a}(\mathrm{i}) .{ }^{*} \mathrm{Wq}\right)+\operatorname{sum}(\mathrm{g} 32\right.\right.$

$31 \mathrm{~b}(\mathrm{i}) . * \mathrm{Wq})))$

$\left(\mathrm{L}(2,1) *\left(\operatorname{sum}\left(\mathrm{g} 3232 \mathrm{a}(\mathrm{i}) .{ }^{*} \mathrm{Wq}\right)+\operatorname{sum}\left(\mathrm{g} 3232 \mathrm{~b}(\mathrm{i}) \cdot{ }^{*} \mathrm{Wq}\right)+\operatorname{sum}\left(\mathrm{g} 3232 \mathrm{a}(\mathrm{i}) \cdot{ }^{*} \mathrm{Wq}\right)+\operatorname{sum}(\mathrm{g} 32\right.\right.$

$\left.\left.\left.32 \mathrm{~b}(\mathrm{i}) \cdot{ }^{*} \mathrm{Wq}\right)\right)\right)$

$\left(\mathrm{L}(4,1) *\left(\operatorname{sum}\left(\mathrm{g} 3233 \mathrm{a}(\mathrm{i}) .{ }^{*} \mathrm{Wq}\right)+\operatorname{sum}\left(\mathrm{g} 3233 \mathrm{~b}(\mathrm{i}) .{ }^{*} \mathrm{Wq}\right)+\operatorname{sum}\left(\mathrm{g} 3233 \mathrm{a}(\mathrm{i}) .{ }^{*} \mathrm{Wq}\right)+\operatorname{sum}(\mathrm{g} 32\right.\right.$

$33 \mathrm{~b}(\mathrm{i}) . * \mathrm{Wq})))$

$\left(\mathrm{L}(5,1) *\left(\operatorname{sum}\left(\mathrm{g} 3331 \mathrm{a}(\mathrm{i}) .{ }^{*} \mathrm{Wq}\right)+\operatorname{sum}\left(\mathrm{g} 3331 \mathrm{~b}(\mathrm{i}) .{ }^{*} \mathrm{Wq}\right)+\operatorname{sum}\left(\mathrm{g} 3331 \mathrm{a}(\mathrm{i}) .{ }^{*} \mathrm{Wq}\right)+\operatorname{sum}(\mathrm{g} 33\right.\right.$

$31 \mathrm{~b}(\mathrm{i}) . * \mathrm{Wq})))$

$\left(\mathrm{L}(7,1) *\left(\operatorname{sum}\left(\mathrm{g} 3332 \mathrm{a}(\mathrm{i}) .{ }^{*} \mathrm{Wq}\right)+\operatorname{sum}\left(\mathrm{g} 3332 \mathrm{~b}(\mathrm{i}) \cdot{ }^{*} \mathrm{Wq}\right)+\operatorname{sum}\left(\mathrm{g} 3332 \mathrm{a}(\mathrm{i}) \cdot{ }^{*} \mathrm{Wq}\right)+\operatorname{sum}(\mathrm{g} 33\right.\right.$

$32 \mathrm{~b}(\mathrm{i}) . * \mathrm{Wq})))$

$\left(\mathrm{L}(3,1) *\left(\operatorname{sum}\left(\mathrm{g} 3333 \mathrm{a}(\mathrm{i}) .{ }^{*} \mathrm{Wq}\right)+\operatorname{sum}\left(\mathrm{g} 3333 \mathrm{~b}(\mathrm{i}) .{ }^{*} \mathrm{Wq}\right)+\operatorname{sum}(\mathrm{g} 3333 \mathrm{a}(\mathrm{i}) . * \mathrm{Wq})+\operatorname{sum}(\mathrm{g} 33\right.\right.$

$33 \mathrm{~b}(\mathrm{i}) \cdot * \mathrm{Wq})))) /(8 * \mathrm{pi})$

$\mathrm{S} 3322$

$\left(\left(\mathrm{L}(1,2) *\left(\operatorname{sum}\left(\mathrm{g} 3131 \mathrm{a}(\mathrm{i}) .{ }^{*} \mathrm{Wq}\right)+\operatorname{sum}\left(\mathrm{g} 3131 \mathrm{~b}(\mathrm{i}) .{ }^{*} \mathrm{Wq}\right)+\operatorname{sum}\left(\mathrm{g} 3131 \mathrm{a}(\mathrm{i}) .{ }^{*} \mathrm{Wq}\right)+\operatorname{sum}(\mathrm{g} 3\right.\right.\right.$ $\left.\left.\left.131 \mathrm{~b}(\mathrm{i}) *{ }^{*} \mathrm{Wq}\right)\right)\right)$

$\left(\mathrm{L}(6,2) *\left(\operatorname{sum}\left(\mathrm{g} 3132 \mathrm{a}(\mathrm{i}) .{ }^{*} \mathrm{Wq}\right)+\operatorname{sum}\left(\mathrm{g} 3132 \mathrm{~b}(\mathrm{i}) .{ }^{*} \mathrm{Wq}\right)+\operatorname{sum}(\mathrm{g} 3132 \mathrm{a}(\mathrm{i}) . * \mathrm{Wq})+\operatorname{sum}(\mathrm{g} 31\right.\right.$ 
$32 \mathrm{~b}(\mathrm{i}) \cdot * \mathrm{Wq})))$

$\left(\mathrm{L}(8,2) *\left(\operatorname{sum}\left(\mathrm{g} 3133 \mathrm{a}(\mathrm{i}) .{ }^{*} \mathrm{Wq}\right)+\operatorname{sum}\left(\mathrm{g} 3133 \mathrm{~b}(\mathrm{i}) .{ }^{*} \mathrm{Wq}\right)+\operatorname{sum}\left(\mathrm{g} 3133 \mathrm{a}(\mathrm{i}) .{ }^{*} \mathrm{Wq}\right)+\operatorname{sum}(\mathrm{g} 31\right.\right.$

$\left.\left.\left.33 \mathrm{~b}(\mathrm{i}) *{ }^{*} \mathrm{Wq}\right)\right)\right)$

$\left(\mathrm{L}(9,2) *\left(\operatorname{sum}\left(\mathrm{g} 3231 \mathrm{a}(\mathrm{i}) .{ }^{*} \mathrm{Wq}\right)+\operatorname{sum}\left(\mathrm{g} 3231 \mathrm{~b}(\mathrm{i}) .{ }^{*} \mathrm{Wq}\right)+\operatorname{sum}\left(\mathrm{g} 3231 \mathrm{a}(\mathrm{i}) .{ }^{*} \mathrm{Wq}\right)+\operatorname{sum}(\mathrm{g} 32\right.\right.$

$\left.\left.31 \mathrm{~b}(\mathrm{i}) \cdot{ }^{*} \mathrm{Wq}\right)\right)$ )

$\left(\mathrm{L}(2,2) *\left(\operatorname{sum}(\mathrm{g} 3232 \mathrm{a}(\mathrm{i}) . * \mathrm{Wq})+\operatorname{sum}\left(\mathrm{g} 3232 \mathrm{~b}(\mathrm{i}) \cdot{ }^{*} \mathrm{Wq}\right)+\operatorname{sum}\left(\mathrm{g} 3232 \mathrm{a}(\mathrm{i}) \cdot{ }^{*} \mathrm{Wq}\right)+\operatorname{sum}(\mathrm{g} 32\right.\right.$

$32 \mathrm{~b}(\mathrm{i}) . * \mathrm{Wq})))$

$\left(\mathrm{L}(4,2) *\left(\operatorname{sum}\left(\mathrm{g} 3233 \mathrm{a}(\mathrm{i}) .{ }^{*} \mathrm{Wq}\right)+\operatorname{sum}\left(\mathrm{g} 3233 \mathrm{~b}(\mathrm{i}) .{ }^{*} \mathrm{Wq}\right)+\operatorname{sum}\left(\mathrm{g} 3233 \mathrm{a}(\mathrm{i}) .{ }^{*} \mathrm{Wq}\right)+\operatorname{sum}(\mathrm{g} 32\right.\right.$

$33 b(\mathrm{i}) . * \mathrm{Wq}))$

$\left(\mathrm{L}(5,2) *\left(\operatorname{sum}\left(\mathrm{g} 3331 \mathrm{a}(\mathrm{i}) .{ }^{*} \mathrm{Wq}\right)+\operatorname{sum}\left(\mathrm{g} 3331 \mathrm{~b}(\mathrm{i}) \cdot{ }^{*} \mathrm{Wq}\right)+\operatorname{sum}\left(\mathrm{g} 3331 \mathrm{a}(\mathrm{i}) \cdot{ }^{*} \mathrm{Wq}\right)+\operatorname{sum}(\mathrm{g} 33\right.\right.$

$31 \mathrm{~b}(\mathrm{i}) . * \mathrm{Wq})))$

$\left(\mathrm{L}(7,2) *\left(\operatorname{sum}(\mathrm{g} 3332 \mathrm{a}(\mathrm{i}) . * \mathrm{Wq})+\operatorname{sum}\left(\mathrm{g} 3332 \mathrm{~b}(\mathrm{i}) \cdot{ }^{*} \mathrm{Wq}\right)+\operatorname{sum}(\mathrm{g} 3332 \mathrm{a}(\mathrm{i}) . * \mathrm{Wq})+\operatorname{sum}(\mathrm{g} 33\right.\right.$

$32 \mathrm{~b}(\mathrm{i}) . * \mathrm{Wq})))$

$\left(\mathrm{L}(3,2) *\left(\operatorname{sum}\left(\mathrm{g} 3333 \mathrm{a}(\mathrm{i}) .{ }^{*} \mathrm{Wq}\right)+\operatorname{sum}\left(\mathrm{g} 3333 \mathrm{~b}(\mathrm{i}) \cdot{ }^{*} \mathrm{Wq}\right)+\operatorname{sum}\left(\mathrm{g} 3333 \mathrm{a}(\mathrm{i}) .{ }^{*} \mathrm{Wq}\right)+\operatorname{sum}(\mathrm{g} 33\right.\right.$

$\left.\left.\left.\left.33 \mathrm{~b}(\mathrm{i}) \cdot{ }^{*} \mathrm{Wq}\right)\right)\right)\right) /(8 * \mathrm{pi})$;

S3333

$\left(\left(\mathrm{L}(1,3) *\left(\operatorname{sum}\left(\mathrm{g} 3131 \mathrm{a}(\mathrm{i}) .{ }^{*} \mathrm{Wq}\right)+\operatorname{sum}\left(\mathrm{g} 3131 \mathrm{~b}(\mathrm{i}) .{ }^{*} \mathrm{Wq}\right)+\operatorname{sum}\left(\mathrm{g} 3131 \mathrm{a}(\mathrm{i}) .{ }^{*} \mathrm{Wq}\right)+\operatorname{sum}(\mathrm{g} 3\right.\right.\right.$ $\left.\left.\left.131 \mathrm{~b}(\mathrm{i}) *{ }^{*} \mathrm{Wq}\right)\right)\right)$

$\left(\mathrm{L}(6,3) *\left(\operatorname{sum}\left(\mathrm{g} 3132 \mathrm{a}(\mathrm{i}) .{ }^{*} \mathrm{Wq}\right)+\operatorname{sum}\left(\mathrm{g} 3132 \mathrm{~b}(\mathrm{i}) \cdot{ }^{*} \mathrm{Wq}\right)+\operatorname{sum}\left(\mathrm{g} 3132 \mathrm{a}(\mathrm{i}) .{ }^{*} \mathrm{Wq}\right)+\operatorname{sum}(\mathrm{g} 31\right.\right.$

$32 \mathrm{~b}(\mathrm{i}) . * \mathrm{Wq})))$

$\left(\mathrm{L}(8,3) *\left(\operatorname{sum}\left(\mathrm{g} 3133 \mathrm{a}(\mathrm{i}) .{ }^{*} \mathrm{Wq}\right)+\operatorname{sum}\left(\mathrm{g} 3133 \mathrm{~b}(\mathrm{i}) \cdot{ }^{*} \mathrm{Wq}\right)+\operatorname{sum}\left(\mathrm{g} 3133 \mathrm{a}(\mathrm{i}) .{ }^{*} \mathrm{Wq}\right)+\operatorname{sum}(\mathrm{g} 31\right.\right.$

$33 \mathrm{~b}(\mathrm{i}) . * \mathrm{Wq})))$

$\left(\mathrm{L}(9,3) *\left(\operatorname{sum}\left(\mathrm{g} 3231 \mathrm{a}(\mathrm{i}) .{ }^{*} \mathrm{Wq}\right)+\operatorname{sum}\left(\mathrm{g} 3231 \mathrm{~b}(\mathrm{i}) .{ }^{*} \mathrm{Wq}\right)+\operatorname{sum}\left(\mathrm{g} 3231 \mathrm{a}(\mathrm{i}) .{ }^{*} \mathrm{Wq}\right)+\operatorname{sum}(\mathrm{g} 32\right.\right.$

$31 \mathrm{~b}(\mathrm{i}) . * \mathrm{Wq})))$

$\left(\mathrm{L}(2,3) *\left(\operatorname{sum}\left(\mathrm{g} 3232 \mathrm{a}(\mathrm{i}) .{ }^{*} \mathrm{Wq}\right)+\operatorname{sum}\left(\mathrm{g} 3232 \mathrm{~b}(\mathrm{i}) \cdot{ }^{*} \mathrm{Wq}\right)+\operatorname{sum}\left(\mathrm{g} 3232 \mathrm{a}(\mathrm{i}) \cdot{ }^{*} \mathrm{Wq}\right)+\operatorname{sum}(\mathrm{g} 32\right.\right.$

$32 \mathrm{~b}(\mathrm{i}) . * \mathrm{Wq})))$

$\left(\mathrm{L}(4,3) *\left(\operatorname{sum}\left(\mathrm{g} 3233 \mathrm{a}(\mathrm{i}) .{ }^{*} \mathrm{Wq}\right)+\operatorname{sum}\left(\mathrm{g} 3233 \mathrm{~b}(\mathrm{i}) \cdot{ }^{*} \mathrm{Wq}\right)+\operatorname{sum}\left(\mathrm{g} 3233 \mathrm{a}(\mathrm{i}) .{ }^{*} \mathrm{Wq}\right)+\operatorname{sum}(\mathrm{g} 32\right.\right.$

$33 b(\mathrm{i}) . * \mathrm{Wq}))$

$\left(\mathrm{L}(5,3) *\left(\operatorname{sum}\left(\mathrm{g} 3331 \mathrm{a}(\mathrm{i}) .{ }^{*} \mathrm{Wq}\right)+\operatorname{sum}\left(\mathrm{g} 3331 \mathrm{~b}(\mathrm{i}) \cdot{ }^{*} \mathrm{Wq}\right)+\operatorname{sum}\left(\mathrm{g} 3331 \mathrm{a}(\mathrm{i}) .{ }^{*} \mathrm{Wq}\right)+\operatorname{sum}(\mathrm{g} 33\right.\right.$

$31 \mathrm{~b}(\mathrm{i}) . * \mathrm{Wq})))$

$\left(\mathrm{L}(7,3) *\left(\operatorname{sum}\left(\mathrm{g} 3332 \mathrm{a}(\mathrm{i}) .{ }^{*} \mathrm{Wq}\right)+\operatorname{sum}\left(\mathrm{g} 3332 \mathrm{~b}(\mathrm{i}) .{ }^{*} \mathrm{Wq}\right)+\operatorname{sum}(\mathrm{g} 3332 \mathrm{a}(\mathrm{i}) . * \mathrm{Wq})+\operatorname{sum}(\mathrm{g} 33\right.\right.$

$\left.\left.\left.32 \mathrm{~b}(\mathrm{i}) \cdot{ }^{*} \mathrm{Wq}\right)\right)\right)$

$\left(\mathrm{L}(3,3) *\left(\operatorname{sum}\left(\mathrm{g} 3333 \mathrm{a}(\mathrm{i}) .{ }^{*} \mathrm{Wq}\right)+\operatorname{sum}\left(\mathrm{g} 3333 \mathrm{~b}(\mathrm{i}) \cdot{ }^{*} \mathrm{Wq}\right)+\operatorname{sum}\left(\mathrm{g} 3333 \mathrm{a}(\mathrm{i}) .{ }^{*} \mathrm{Wq}\right)+\operatorname{sum}(\mathrm{g} 33\right.\right.$

$\left.\left.\left.\left.33 \mathrm{~b}(\mathrm{i}) *{ }^{*} \mathrm{Wq}\right)\right)\right)\right) /(8 * \mathrm{pi})$;

S3323

$\left(\left(\mathrm{L}(1,4) *\left(\operatorname{sum}(\mathrm{g} 3131 \mathrm{a}(\mathrm{i}) . * \mathrm{Wq})+\operatorname{sum}(\mathrm{g} 3131 \mathrm{~b}(\mathrm{i}) . * \mathrm{Wq})+\operatorname{sum}\left(\mathrm{g} 3131 \mathrm{a}(\mathrm{i}) .{ }^{*} \mathrm{Wq}\right)+\operatorname{sum}(\mathrm{g} 3\right.\right.\right.$ $131 \mathrm{~b}(\mathrm{i}) * \mathrm{Wq})))$

$\left(\mathrm{L}(6,4) *\left(\operatorname{sum}\left(\mathrm{g} 3132 \mathrm{a}(\mathrm{i}) .{ }^{*} \mathrm{Wq}\right)+\operatorname{sum}\left(\mathrm{g} 3132 \mathrm{~b}(\mathrm{i}) \cdot{ }^{*} \mathrm{Wq}\right)+\operatorname{sum}\left(\mathrm{g} 3132 \mathrm{a}(\mathrm{i}) .{ }^{*} \mathrm{Wq}\right)+\operatorname{sum}(\mathrm{g} 31\right.\right.$ $\left.\left.32 \mathrm{~b}(\mathrm{i}){ }^{*} \mathrm{Wq}\right)\right)$ )

$\left(\mathrm{L}(8,4) *\left(\operatorname{sum}\left(\mathrm{g} 3133 \mathrm{a}(\mathrm{i}) .{ }^{*} \mathrm{Wq}\right)+\operatorname{sum}\left(\mathrm{g} 3133 \mathrm{~b}(\mathrm{i}) .{ }^{*} \mathrm{Wq}\right)+\operatorname{sum}\left(\mathrm{g} 3133 \mathrm{a}(\mathrm{i}) .{ }^{*} \mathrm{Wq}\right)+\operatorname{sum}(\mathrm{g} 31\right.\right.$

$33 \mathrm{~b}(\mathrm{i}) \cdot * \mathrm{Wq}))$

$\left(\mathrm{L}(9,4) *\left(\operatorname{sum}(\mathrm{g} 3231 \mathrm{a}(\mathrm{i}) . * \mathrm{Wq})+\operatorname{sum}\left(\mathrm{g} 3231 \mathrm{~b}(\mathrm{i}) \cdot{ }^{*} \mathrm{Wq}\right)+\operatorname{sum}\left(\mathrm{g} 3231 \mathrm{a}(\mathrm{i}) \cdot{ }^{*} \mathrm{Wq}\right)+\operatorname{sum}(\mathrm{g} 32\right.\right.$

$\left.\left.\left.31 \mathrm{~b}(\mathrm{i}) \cdot{ }^{*} \mathrm{Wq}\right)\right)\right)$ 
$\left(\mathrm{L}(2,4) *\left(\operatorname{sum}\left(\mathrm{g} 3232 \mathrm{a}(\mathrm{i}) \cdot{ }^{*} \mathrm{Wq}\right)+\operatorname{sum}(\mathrm{g} 3232 \mathrm{~b}(\mathrm{i}) \cdot * \mathrm{Wq})+\operatorname{sum}\left(\mathrm{g} 3232 \mathrm{a}(\mathrm{i}) \cdot{ }^{*} \mathrm{Wq}\right)+\operatorname{sum}(\mathrm{g} 32\right.\right.$ $32 \mathrm{~b}(\mathrm{i}) . * \mathrm{Wq}))$ ) $\left(\mathrm{L}(4,4) *\left(\operatorname{sum}(\mathrm{g} 3233 \mathrm{a}(\mathrm{i}) . * \mathrm{Wq})+\operatorname{sum}\left(\mathrm{g} 3233 \mathrm{~b}(\mathrm{i}) .{ }^{*} \mathrm{Wq}\right)+\operatorname{sum}\left(\mathrm{g} 3233 \mathrm{a}(\mathrm{i}) .{ }^{*} \mathrm{Wq}\right)+\operatorname{sum}(\mathrm{g} 32\right.\right.$ $33 \mathrm{~b}(\mathrm{i}) . * \mathrm{Wq})))$ $\left(\mathrm{L}(5,4) *\left(\operatorname{sum}\left(\mathrm{g} 3331 \mathrm{a}(\mathrm{i}) .{ }^{*} \mathrm{Wq}\right)+\operatorname{sum}\left(\mathrm{g} 3331 \mathrm{~b}(\mathrm{i}) \cdot{ }^{*} \mathrm{Wq}\right)+\operatorname{sum}\left(\mathrm{g} 3331 \mathrm{a}(\mathrm{i}) .{ }^{*} \mathrm{Wq}\right)+\operatorname{sum}(\mathrm{g} 33\right.\right.$ $31 \mathrm{~b}(\mathrm{i}) . * \mathrm{Wq})))$ $\left(\mathrm{L}(7,4) *\left(\operatorname{sum}\left(\mathrm{g} 3332 \mathrm{a}(\mathrm{i}) .{ }^{*} \mathrm{Wq}\right)+\operatorname{sum}\left(\mathrm{g} 3332 \mathrm{~b}(\mathrm{i}) .{ }^{*} \mathrm{Wq}\right)+\operatorname{sum}\left(\mathrm{g} 3332 \mathrm{a}(\mathrm{i}) .{ }^{*} \mathrm{Wq}\right)+\operatorname{sum}(\mathrm{g} 33\right.\right.$ $32 \mathrm{~b}(\mathrm{i}) . * \mathrm{Wq})))$ $\left(\mathrm{L}(3,4) *\left(\operatorname{sum}\left(\mathrm{g} 3333 \mathrm{a}(\mathrm{i}) .{ }^{*} \mathrm{Wq}\right)+\operatorname{sum}\left(\mathrm{g} 3333 \mathrm{~b}(\mathrm{i}) .{ }^{*} \mathrm{Wq}\right)+\operatorname{sum}(\mathrm{g} 3333 \mathrm{a}(\mathrm{i}) . * \mathrm{Wq})+\operatorname{sum}(\mathrm{g} 33\right.\right.$ $33 \mathrm{~b}(\mathrm{i}) \cdot * \mathrm{Wq})))) /(8 * \mathrm{pi})$;

$\mathrm{S} 3331$

$\left(\left(\mathrm{L}(1,5) *\left(\operatorname{sum}\left(\mathrm{g} 3131 \mathrm{a}(\mathrm{i}) .{ }^{*} \mathrm{Wq}\right)+\operatorname{sum}\left(\mathrm{g} 3131 \mathrm{~b}(\mathrm{i}) .{ }^{*} \mathrm{Wq}\right)+\operatorname{sum}\left(\mathrm{g} 3131 \mathrm{a}(\mathrm{i}) .{ }^{*} \mathrm{Wq}\right)+\operatorname{sum}(\mathrm{g} 3\right.\right.\right.$ $\left.\left.\left.131 \mathrm{~b}(\mathrm{i}) \cdot{ }^{*} \mathrm{Wq}\right)\right)\right)$

$\left(\mathrm{L}(6,5) *\left(\operatorname{sum}\left(\mathrm{g} 3132 \mathrm{a}(\mathrm{i}) .{ }^{*} \mathrm{Wq}\right)+\operatorname{sum}\left(\mathrm{g} 3132 \mathrm{~b}(\mathrm{i}) \cdot{ }^{*} \mathrm{Wq}\right)+\operatorname{sum}(\mathrm{g} 3132 \mathrm{a}(\mathrm{i}) . * \mathrm{Wq})+\operatorname{sum}(\mathrm{g} 31\right.\right.$ $32 \mathrm{~b}(\mathrm{i}) . * \mathrm{Wq}))$ ) $\left(\mathrm{L}(8,5) *\left(\operatorname{sum}\left(\mathrm{g} 3133 \mathrm{a}(\mathrm{i}) .{ }^{*} \mathrm{Wq}\right)+\operatorname{sum}\left(\mathrm{g} 3133 \mathrm{~b}(\mathrm{i}) .{ }^{*} \mathrm{Wq}\right)+\operatorname{sum}(\mathrm{g} 3133 \mathrm{a}(\mathrm{i}) . * \mathrm{Wq})+\operatorname{sum}(\mathrm{g} 31\right.\right.$ $33 \mathrm{~b}(\mathrm{i}) . * \mathrm{Wq}))$ ) $\left(\mathrm{L}(9,5) *\left(\operatorname{sum}\left(\mathrm{g} 3231 \mathrm{a}(\mathrm{i}) .{ }^{*} \mathrm{Wq}\right)+\operatorname{sum}\left(\mathrm{g} 3231 \mathrm{~b}(\mathrm{i}) .{ }^{*} \mathrm{Wq}\right)+\operatorname{sum}\left(\mathrm{g} 3231 \mathrm{a}(\mathrm{i}) \cdot{ }^{*} \mathrm{Wq}\right)+\operatorname{sum}(\mathrm{g} 32\right.\right.$ $31 \mathrm{~b}(\mathrm{i}) . * \mathrm{Wq})))$ $\left(\mathrm{L}(2,5) *\left(\operatorname{sum}\left(\mathrm{g} 3232 \mathrm{a}(\mathrm{i}) .{ }^{*} \mathrm{Wq}\right)+\operatorname{sum}\left(\mathrm{g} 3232 \mathrm{~b}(\mathrm{i}) \cdot{ }^{*} \mathrm{Wq}\right)+\operatorname{sum}\left(\mathrm{g} 3232 \mathrm{a}(\mathrm{i}) \cdot{ }^{*} \mathrm{Wq}\right)+\operatorname{sum}(\mathrm{g} 32\right.\right.$ $32 \mathrm{~b}(\mathrm{i}) . * \mathrm{Wq})))$ $\left(\mathrm{L}(4,5) *\left(\operatorname{sum}(\mathrm{g} 3233 \mathrm{a}(\mathrm{i}) . * \mathrm{Wq})+\operatorname{sum}\left(\mathrm{g} 3233 \mathrm{~b}(\mathrm{i}) \cdot{ }^{*} \mathrm{Wq}\right)+\operatorname{sum}\left(\mathrm{g} 3233 \mathrm{a}(\mathrm{i}) \cdot{ }^{*} \mathrm{Wq}\right)+\operatorname{sum}(\mathrm{g} 32\right.\right.$ $33 \mathrm{~b}(\mathrm{i}) . * \mathrm{Wq})))$ $\left(\mathrm{L}(5,5) *\left(\operatorname{sum}\left(\mathrm{g} 3331 \mathrm{a}(\mathrm{i}) .{ }^{*} \mathrm{Wq}\right)+\operatorname{sum}\left(\mathrm{g} 3331 \mathrm{~b}(\mathrm{i}) \cdot{ }^{*} \mathrm{Wq}\right)+\operatorname{sum}\left(\mathrm{g} 3331 \mathrm{a}(\mathrm{i}) .{ }^{*} \mathrm{Wq}\right)+\operatorname{sum}(\mathrm{g} 33\right.\right.$ $31 \mathrm{~b}(\mathrm{i}) . * \mathrm{Wq})))$ $\left(\mathrm{L}(7,5) *\left(\operatorname{sum}\left(\mathrm{g} 3332 \mathrm{a}(\mathrm{i}) .{ }^{*} \mathrm{Wq}\right)+\operatorname{sum}\left(\mathrm{g} 3332 \mathrm{~b}(\mathrm{i}) .{ }^{*} \mathrm{Wq}\right)+\operatorname{sum}\left(\mathrm{g} 3332 \mathrm{a}(\mathrm{i}) .{ }^{*} \mathrm{Wq}\right)+\operatorname{sum}(\mathrm{g} 33\right.\right.$ $32 \mathrm{~b}(\mathrm{i}) . * \mathrm{Wq})))$ $\left(\mathrm{L}(3,5) *\left(\operatorname{sum}\left(\mathrm{g} 3333 \mathrm{a}(\mathrm{i}) .{ }^{*} \mathrm{Wq}\right)+\operatorname{sum}\left(\mathrm{g} 3333 \mathrm{~b}(\mathrm{i}) .{ }^{*} \mathrm{Wq}\right)+\operatorname{sum}\left(\mathrm{g} 3333 \mathrm{a}(\mathrm{i}) .{ }^{*} \mathrm{Wq}\right)+\operatorname{sum}(\mathrm{g} 33\right.\right.$ $33 \mathrm{~b}(\mathrm{i}) . * \mathrm{Wq})))) /(8 * \mathrm{pi})$;

$\mathrm{S} 3312$

$\left(\left(\mathrm{L}(1,6) *\left(\operatorname{sum}\left(\mathrm{g} 3131 \mathrm{a}(\mathrm{i}) .{ }^{*} \mathrm{Wq}\right)+\operatorname{sum}\left(\mathrm{g} 3131 \mathrm{~b}(\mathrm{i}) .{ }^{*} \mathrm{Wq}\right)+\operatorname{sum}\left(\mathrm{g} 3131 \mathrm{a}(\mathrm{i}) .{ }^{*} \mathrm{Wq}\right)+\operatorname{sum}(\mathrm{g} 3\right.\right.\right.$ $131 b(\mathrm{i}) * \mathrm{Wq}))$ $\left(\mathrm{L}(6,6) *\left(\operatorname{sum}\left(\mathrm{g} 3132 \mathrm{a}(\mathrm{i}) .{ }^{*} \mathrm{Wq}\right)+\operatorname{sum}\left(\mathrm{g} 3132 \mathrm{~b}(\mathrm{i}) \cdot{ }^{*} \mathrm{Wq}\right)+\operatorname{sum}(\mathrm{g} 3132 \mathrm{a}(\mathrm{i}) . * \mathrm{Wq})+\operatorname{sum}(\mathrm{g} 31\right.\right.$ $32 \mathrm{~b}(\mathrm{i}) . * \mathrm{Wq})))$ $\left(\mathrm{L}(8,6) *\left(\operatorname{sum}\left(\mathrm{g} 3133 \mathrm{a}(\mathrm{i}) .{ }^{*} \mathrm{Wq}\right)+\operatorname{sum}\left(\mathrm{g} 3133 \mathrm{~b}(\mathrm{i}) .{ }^{*} \mathrm{Wq}\right)+\operatorname{sum}\left(\mathrm{g} 3133 \mathrm{a}(\mathrm{i}) .{ }^{*} \mathrm{Wq}\right)+\operatorname{sum}(\mathrm{g} 31\right.\right.$ $33 \mathrm{~b}(\mathrm{i}) . * \mathrm{Wq})))$ $\left(\mathrm{L}(9,6) *\left(\operatorname{sum}\left(\mathrm{g} 3231 \mathrm{a}(\mathrm{i}) .{ }^{*} \mathrm{Wq}\right)+\operatorname{sum}\left(\mathrm{g} 3231 \mathrm{~b}(\mathrm{i}) .{ }^{*} \mathrm{Wq}\right)+\operatorname{sum}\left(\mathrm{g} 3231 \mathrm{a}(\mathrm{i}) \cdot{ }^{*} \mathrm{Wq}\right)+\operatorname{sum}(\mathrm{g} 32\right.\right.$ $31 \mathrm{~b}(\mathrm{i}) . * \mathrm{Wq})))$ $\left(\mathrm{L}(2,6) *\left(\operatorname{sum}\left(\mathrm{g} 3232 \mathrm{a}(\mathrm{i}) .{ }^{*} \mathrm{Wq}\right)+\operatorname{sum}\left(\mathrm{g} 3232 \mathrm{~b}(\mathrm{i}) \cdot{ }^{*} \mathrm{Wq}\right)+\operatorname{sum}\left(\mathrm{g} 3232 \mathrm{a}(\mathrm{i}) .{ }^{*} \mathrm{Wq}\right)+\operatorname{sum}(\mathrm{g} 32\right.\right.$ $32 \mathrm{~b}(\mathrm{i}) . * \mathrm{Wq})))$ $\left(\mathrm{L}(4,6) *\left(\operatorname{sum}(\mathrm{g} 3233 \mathrm{a}(\mathrm{i}) . * \mathrm{Wq})+\operatorname{sum}\left(\mathrm{g} 3233 \mathrm{~b}(\mathrm{i}) \cdot{ }^{*} \mathrm{Wq}\right)+\operatorname{sum}\left(\mathrm{g} 3233 \mathrm{a}(\mathrm{i}) \cdot{ }^{*} \mathrm{Wq}\right)+\operatorname{sum}(\mathrm{g} 32\right.\right.$ $33 \mathrm{~b}(\mathrm{i}) . * \mathrm{Wq})))$ $\left(\mathrm{L}(5,6) *\left(\operatorname{sum}\left(\mathrm{g} 3331 \mathrm{a}(\mathrm{i}) .{ }^{*} \mathrm{Wq}\right)+\operatorname{sum}\left(\mathrm{g} 3331 \mathrm{~b}(\mathrm{i}) .{ }^{*} \mathrm{Wq}\right)+\operatorname{sum}\left(\mathrm{g} 3331 \mathrm{a}(\mathrm{i}) .{ }^{*} \mathrm{Wq}\right)+\operatorname{sum}(\mathrm{g} 33\right.\right.$ 
$31 \mathrm{~b}(\mathrm{i}) . * \mathrm{Wq})))$

$\left(\mathrm{L}(7,6) *\left(\operatorname{sum}\left(\mathrm{g} 3332 \mathrm{a}(\mathrm{i}) .{ }^{*} \mathrm{Wq}\right)+\operatorname{sum}\left(\mathrm{g} 3332 \mathrm{~b}(\mathrm{i}) \cdot{ }^{*} \mathrm{Wq}\right)+\operatorname{sum}\left(\mathrm{g} 3332 \mathrm{a}(\mathrm{i}) .{ }^{*} \mathrm{Wq}\right)+\operatorname{sum}(\mathrm{g} 33\right.\right.$

$32 \mathrm{~b}(\mathrm{i}) . * \mathrm{Wq}))$ )

$\left(\mathrm{L}(3,6) *\left(\operatorname{sum}\left(\mathrm{g} 3333 \mathrm{a}(\mathrm{i}) .{ }^{*} \mathrm{Wq}\right)+\operatorname{sum}\left(\mathrm{g} 3333 \mathrm{~b}(\mathrm{i}) \cdot{ }^{*} \mathrm{Wq}\right)+\operatorname{sum}\left(\mathrm{g} 3333 \mathrm{a}(\mathrm{i}) .{ }^{*} \mathrm{Wq}\right)+\operatorname{sum}(\mathrm{g} 33\right.\right.$

$33 \mathrm{~b}(\mathrm{i}) *$ (Wq) $))) /(8 * \mathrm{pi})$;

$\mathrm{S} 3332$

$\left(\left(\mathrm{L}(1,7) *\left(\operatorname{sum}\left(\mathrm{g} 3131 \mathrm{a}(\mathrm{i}) .{ }^{*} \mathrm{Wq}\right)+\operatorname{sum}\left(\mathrm{g} 3131 \mathrm{~b}(\mathrm{i}) .{ }^{*} \mathrm{Wq}\right)+\operatorname{sum}\left(\mathrm{g} 3131 \mathrm{a}(\mathrm{i}) .{ }^{*} \mathrm{Wq}\right)+\operatorname{sum}(\mathrm{g} 3\right.\right.\right.$ $\left.\left.\left.131 \mathrm{~b}(\mathrm{i}) \cdot{ }^{*} \mathrm{Wq}\right)\right)\right)$

$\left(\mathrm{L}(6,7) *\left(\operatorname{sum}\left(\mathrm{g} 3132 \mathrm{a}(\mathrm{i}) .{ }^{*} \mathrm{Wq}\right)+\operatorname{sum}\left(\mathrm{g} 3132 \mathrm{~b}(\mathrm{i}) \cdot{ }^{*} \mathrm{Wq}\right)+\operatorname{sum}\left(\mathrm{g} 3132 \mathrm{a}(\mathrm{i}) .{ }^{*} \mathrm{Wq}\right)+\operatorname{sum}(\mathrm{g} 31\right.\right.$ $32 \mathrm{~b}(\mathrm{i}) . * \mathrm{Wq}))$ )

$\left(\mathrm{L}(8,7) *\left(\operatorname{sum}\left(\mathrm{g} 3133 \mathrm{a}(\mathrm{i}) .{ }^{*} \mathrm{Wq}\right)+\operatorname{sum}(\mathrm{g} 3133 \mathrm{~b}(\mathrm{i}) . * \mathrm{Wq})+\operatorname{sum}\left(\mathrm{g} 3133 \mathrm{a}(\mathrm{i}) .{ }^{*} \mathrm{Wq}\right)+\operatorname{sum}(\mathrm{g} 31\right.\right.$ $33 \mathrm{~b}(\mathrm{i}) . * \mathrm{Wq}))$ )

$\left(\mathrm{L}(9,7) *\left(\operatorname{sum}\left(\mathrm{g} 3231 \mathrm{a}(\mathrm{i}) .{ }^{*} \mathrm{Wq}\right)+\operatorname{sum}\left(\mathrm{g} 3231 \mathrm{~b}(\mathrm{i}) .{ }^{*} \mathrm{Wq}\right)+\operatorname{sum}\left(\mathrm{g} 3231 \mathrm{a}(\mathrm{i}) .{ }^{*} \mathrm{Wq}\right)+\operatorname{sum}(\mathrm{g} 32\right.\right.$ $31 \mathrm{~b}(\mathrm{i}) . * \mathrm{Wq}))$ )

$\left(\mathrm{L}(2,7) *\left(\operatorname{sum}\left(\mathrm{g} 3232 \mathrm{a}(\mathrm{i}) .{ }^{*} \mathrm{Wq}\right)+\operatorname{sum}\left(\mathrm{g} 3232 \mathrm{~b}(\mathrm{i}) .{ }^{*} \mathrm{Wq}\right)+\operatorname{sum}\left(\mathrm{g} 3232 \mathrm{a}(\mathrm{i}) .{ }^{*} \mathrm{Wq}\right)+\operatorname{sum}(\mathrm{g} 32\right.\right.$ $32 \mathrm{~b}(\mathrm{i}) . * \mathrm{Wq}))$ )

$\left(\mathrm{L}(4,7) *\left(\operatorname{sum}\left(\mathrm{g} 3233 \mathrm{a}(\mathrm{i}) .{ }^{*} \mathrm{Wq}\right)+\operatorname{sum}(\mathrm{g} 3233 \mathrm{~b}(\mathrm{i}) . * \mathrm{Wq})+\operatorname{sum}\left(\mathrm{g} 3233 \mathrm{a}(\mathrm{i}) .{ }^{*} \mathrm{Wq}\right)+\operatorname{sum}(\mathrm{g} 32\right.\right.$ $33 \mathrm{~b}(\mathrm{i}) . * \mathrm{Wq}))$ )

$\left(\mathrm{L}(5,7) *\left(\operatorname{sum}\left(\mathrm{g} 3331 \mathrm{a}(\mathrm{i}) \cdot{ }^{*} \mathrm{Wq}\right)+\operatorname{sum}\left(\mathrm{g} 3331 \mathrm{~b}(\mathrm{i}) \cdot{ }^{*} \mathrm{Wq}\right)+\operatorname{sum}\left(\mathrm{g} 3331 \mathrm{a}(\mathrm{i}) .{ }^{*} \mathrm{Wq}\right)+\operatorname{sum}(\mathrm{g} 33\right.\right.$ $31 \mathrm{~b}(\mathrm{i}) . * \mathrm{Wq}))$ )

$\left(\mathrm{L}(7,7) *\left(\operatorname{sum}\left(\mathrm{g} 3332 \mathrm{a}(\mathrm{i}) .{ }^{*} \mathrm{Wq}\right)+\operatorname{sum}\left(\mathrm{g} 3332 \mathrm{~b}(\mathrm{i}) .{ }^{*} \mathrm{Wq}\right)+\operatorname{sum}\left(\mathrm{g} 3332 \mathrm{a}(\mathrm{i}) .{ }^{*} \mathrm{Wq}\right)+\operatorname{sum}(\mathrm{g} 33\right.\right.$ $32 \mathrm{~b}(\mathrm{i}) . * \mathrm{Wq})))$

$\left(\mathrm{L}(3,7) *\left(\operatorname{sum}\left(\mathrm{g} 3333 \mathrm{a}(\mathrm{i}) .{ }^{*} \mathrm{Wq}\right)+\operatorname{sum}\left(\mathrm{g} 3333 \mathrm{~b}(\mathrm{i}) \cdot{ }^{*} \mathrm{Wq}\right)+\operatorname{sum}\left(\mathrm{g} 3333 \mathrm{a}(\mathrm{i}) \cdot{ }^{*} \mathrm{Wq}\right)+\operatorname{sum}(\mathrm{g} 33\right.\right.$ $33 \mathrm{~b}(\mathrm{i}) \cdot * \mathrm{Wq})))) /(8 * \mathrm{pi})$;

S3313

$\left(\left(\mathrm{L}(1,8) *\left(\operatorname{sum}\left(\mathrm{g} 3131 \mathrm{a}(\mathrm{i}) .{ }^{*} \mathrm{Wq}\right)+\operatorname{sum}(\mathrm{g} 3131 \mathrm{~b}(\mathrm{i}) . * \mathrm{Wq})+\operatorname{sum}\left(\mathrm{g} 3131 \mathrm{a}(\mathrm{i}) .{ }^{*} \mathrm{Wq}\right)+\operatorname{sum}(\mathrm{g} 3\right.\right.\right.$ $131 \mathrm{~b}(\mathrm{i}) \cdot * \mathrm{Wq})))$

$\left(\mathrm{L}(6,8) *\left(\operatorname{sum}\left(\mathrm{g} 3132 \mathrm{a}(\mathrm{i}) .{ }^{*} \mathrm{Wq}\right)+\operatorname{sum}\left(\mathrm{g} 3132 \mathrm{~b}(\mathrm{i}) .{ }^{*} \mathrm{Wq}\right)+\operatorname{sum}\left(\mathrm{g} 3132 \mathrm{a}(\mathrm{i}) .{ }^{*} \mathrm{Wq}\right)+\operatorname{sum}(\mathrm{g} 31\right.\right.$ $32 \mathrm{~b}(\mathrm{i}) . * \mathrm{Wq}))$ )

$\left(\mathrm{L}(8,8) *\left(\operatorname{sum}\left(\mathrm{g} 3133 \mathrm{a}(\mathrm{i}) .{ }^{*} \mathrm{Wq}\right)+\operatorname{sum}\left(\mathrm{g} 3133 \mathrm{~b}(\mathrm{i}) .{ }^{*} \mathrm{Wq}\right)+\operatorname{sum}\left(\mathrm{g} 3133 \mathrm{a}(\mathrm{i}) .{ }^{*} \mathrm{Wq}\right)+\operatorname{sum}(\mathrm{g} 31\right.\right.$ $33 \mathrm{~b}(\mathrm{i}) . * \mathrm{Wq})))$

$\left(\mathrm{L}(9,8) *\left(\operatorname{sum}\left(\mathrm{g} 3231 \mathrm{a}(\mathrm{i}) .{ }^{*} \mathrm{Wq}\right)+\operatorname{sum}\left(\mathrm{g} 3231 \mathrm{~b}(\mathrm{i}) \cdot{ }^{*} \mathrm{Wq}\right)+\operatorname{sum}\left(\mathrm{g} 3231 \mathrm{a}(\mathrm{i}) .{ }^{*} \mathrm{Wq}\right)+\operatorname{sum}(\mathrm{g} 32\right.\right.$ $31 \mathrm{~b}(\mathrm{i}) . * \mathrm{Wq}))$ )

$\left(\mathrm{L}(2,8) *\left(\operatorname{sum}\left(\mathrm{g} 3232 \mathrm{a}(\mathrm{i}) .{ }^{*} \mathrm{Wq}\right)+\operatorname{sum}\left(\mathrm{g} 3232 \mathrm{~b}(\mathrm{i}) .{ }^{*} \mathrm{Wq}\right)+\operatorname{sum}\left(\mathrm{g} 3232 \mathrm{a}(\mathrm{i}) .{ }^{*} \mathrm{Wq}\right)+\operatorname{sum}(\mathrm{g} 32\right.\right.$ $\left.\left.\left.32 \mathrm{~b}(\mathrm{i}) *{ }^{*} \mathrm{Wq}\right)\right)\right)$

$\left(\mathrm{L}(4,8) *\left(\operatorname{sum}\left(\mathrm{g} 3233 \mathrm{a}(\mathrm{i}) .{ }^{*} \mathrm{Wq}\right)+\operatorname{sum}\left(\mathrm{g} 3233 \mathrm{~b}(\mathrm{i}) \cdot{ }^{*} \mathrm{Wq}\right)+\operatorname{sum}\left(\mathrm{g} 3233 \mathrm{a}(\mathrm{i}) .{ }^{*} \mathrm{Wq}\right)+\operatorname{sum}(\mathrm{g} 32\right.\right.$ $33 \mathrm{~b}(\mathrm{i}) . * \mathrm{Wq}))$ )

$\left(\mathrm{L}(5,8) *\left(\operatorname{sum}\left(\mathrm{g} 3331 \mathrm{a}(\mathrm{i}) .{ }^{*} \mathrm{Wq}\right)+\operatorname{sum}\left(\mathrm{g} 3331 \mathrm{~b}(\mathrm{i}) .{ }^{*} \mathrm{Wq}\right)+\operatorname{sum}\left(\mathrm{g} 3331 \mathrm{a}(\mathrm{i}) .{ }^{*} \mathrm{Wq}\right)+\operatorname{sum}(\mathrm{g} 33\right.\right.$ $31 \mathrm{~b}(\mathrm{i}) . * \mathrm{Wq})))$ $\left(\mathrm{L}(7,8) *\left(\operatorname{sum}\left(\mathrm{g} 3332 \mathrm{a}(\mathrm{i}) .{ }^{*} \mathrm{Wq}\right)+\operatorname{sum}\left(\mathrm{g} 3332 \mathrm{~b}(\mathrm{i}) .{ }^{*} \mathrm{Wq}\right)+\operatorname{sum}\left(\mathrm{g} 3332 \mathrm{a}(\mathrm{i}) .{ }^{*} \mathrm{Wq}\right)+\operatorname{sum}(\mathrm{g} 33\right.\right.$ $32 \mathrm{~b}(\mathrm{i}) . * \mathrm{Wq}))$ ) $\left(\mathrm{L}(3,8) *\left(\operatorname{sum}\left(\mathrm{g} 3333 \mathrm{a}(\mathrm{i}) .{ }^{*} \mathrm{Wq}\right)+\operatorname{sum}\left(\mathrm{g} 3333 \mathrm{~b}(\mathrm{i}) .{ }^{*} \mathrm{Wq}\right)+\operatorname{sum}\left(\mathrm{g} 3333 \mathrm{a}(\mathrm{i}) .{ }^{*} \mathrm{Wq}\right)+\operatorname{sum}(\mathrm{g} 33\right.\right.$ $33 \mathrm{~b}(\mathrm{i}) . * \mathrm{Wq})))) /(8 * \mathrm{pi})$; 
S3321

$\left(\left(\mathrm{L}(1,9) *\left(\operatorname{sum}\left(\mathrm{g} 3131 \mathrm{a}(\mathrm{i}) .{ }^{*} \mathrm{Wq}\right)+\operatorname{sum}\left(\mathrm{g} 3131 \mathrm{~b}(\mathrm{i}){ }^{*} \mathrm{Wq}\right)+\operatorname{sum}\left(\mathrm{g} 3131 \mathrm{a}(\mathrm{i}) .{ }^{*} \mathrm{Wq}\right)+\operatorname{sum}(\mathrm{g} 3\right.\right.\right.$ $\left.\left.\left.131 \mathrm{~b}(\mathrm{i}) \cdot{ }^{*} \mathrm{Wq}\right)\right)\right)$

$\left(\mathrm{L}(6,9) *\left(\operatorname{sum}(\mathrm{g} 3132 \mathrm{a}(\mathrm{i}) . * \mathrm{Wq})+\operatorname{sum}\left(\mathrm{g} 3132 \mathrm{~b}(\mathrm{i}) \cdot{ }^{*} \mathrm{Wq}\right)+\operatorname{sum}\left(\mathrm{g} 3132 \mathrm{a}(\mathrm{i}) \cdot{ }^{*} \mathrm{Wq}\right)+\operatorname{sum}(\mathrm{g} 31\right.\right.$ $32 \mathrm{~b}(\mathrm{i}) . * \mathrm{Wq})))$

$\left(\mathrm{L}(8,9) *\left(\operatorname{sum}\left(\mathrm{g} 3133 \mathrm{a}(\mathrm{i}) .{ }^{*} \mathrm{Wq}\right)+\operatorname{sum}\left(\mathrm{g} 3133 \mathrm{~b}(\mathrm{i}) .{ }^{*} \mathrm{Wq}\right)+\operatorname{sum}\left(\mathrm{g} 3133 \mathrm{a}(\mathrm{i}) .{ }^{*} \mathrm{Wq}\right)+\operatorname{sum}(\mathrm{g} 31\right.\right.$ $33 \mathrm{~b}(\mathrm{i}) . * \mathrm{Wq}))$ )

$\left(\mathrm{L}(9,9) *\left(\operatorname{sum}\left(\mathrm{g} 3231 \mathrm{a}(\mathrm{i}) .{ }^{*} \mathrm{Wq}\right)+\operatorname{sum}\left(\mathrm{g} 3231 \mathrm{~b}(\mathrm{i}) \cdot{ }^{*} \mathrm{Wq}\right)+\operatorname{sum}\left(\mathrm{g} 3231 \mathrm{a}(\mathrm{i}) .{ }^{*} \mathrm{Wq}\right)+\operatorname{sum}(\mathrm{g} 32\right.\right.$

$31 \mathrm{~b}(\mathrm{i}) . * \mathrm{Wq})))$

$\left(\mathrm{L}(2,9) *\left(\operatorname{sum}(\mathrm{g} 3232 \mathrm{a}(\mathrm{i}) . * \mathrm{Wq})+\operatorname{sum}\left(\mathrm{g} 3232 \mathrm{~b}(\mathrm{i}) \cdot{ }^{*} \mathrm{Wq}\right)+\operatorname{sum}\left(\mathrm{g} 3232 \mathrm{a}(\mathrm{i}) \cdot{ }^{*} \mathrm{Wq}\right)+\operatorname{sum}(\mathrm{g} 32\right.\right.$

$32 \mathrm{~b}(\mathrm{i}) . * \mathrm{Wq})))$

$\left(\mathrm{L}(4,9) *\left(\operatorname{sum}\left(\mathrm{g} 3233 \mathrm{a}(\mathrm{i}) .{ }^{*} \mathrm{Wq}\right)+\operatorname{sum}\left(\mathrm{g} 3233 \mathrm{~b}(\mathrm{i}) .{ }^{*} \mathrm{Wq}\right)+\operatorname{sum}\left(\mathrm{g} 3233 \mathrm{a}(\mathrm{i}) .{ }^{*} \mathrm{Wq}\right)+\operatorname{sum}(\mathrm{g} 32\right.\right.$

$33 \mathrm{~b}(\mathrm{i}) . * \mathrm{Wq}))$ )

$\left(\mathrm{L}(5,9) *\left(\operatorname{sum}\left(\mathrm{g} 3331 \mathrm{a}(\mathrm{i}) .{ }^{*} \mathrm{Wq}\right)+\operatorname{sum}\left(\mathrm{g} 3331 \mathrm{~b}(\mathrm{i}) .{ }^{*} \mathrm{Wq}\right)+\operatorname{sum}\left(\mathrm{g} 3331 \mathrm{a}(\mathrm{i}) .{ }^{*} \mathrm{Wq}\right)+\operatorname{sum}(\mathrm{g} 33\right.\right.$

$31 \mathrm{~b}(\mathrm{i}) . * \mathrm{Wq})))$

$\left(\mathrm{L}(7,9) *\left(\operatorname{sum}(\mathrm{g} 3332 \mathrm{a}(\mathrm{i}) . * \mathrm{Wq})+\operatorname{sum}\left(\mathrm{g} 3332 \mathrm{~b}(\mathrm{i}) \cdot{ }^{*} \mathrm{Wq}\right)+\operatorname{sum}\left(\mathrm{g} 3332 \mathrm{a}(\mathrm{i}) \cdot{ }^{*} \mathrm{Wq}\right)+\operatorname{sum}(\mathrm{g} 33\right.\right.$

$32 \mathrm{~b}(\mathrm{i}) . * \mathrm{Wq}))$ )

$\left(\mathrm{L}(3,9) *\left(\operatorname{sum}\left(\mathrm{g} 3333 \mathrm{a}(\mathrm{i}) .{ }^{*} \mathrm{Wq}\right)+\operatorname{sum}\left(\mathrm{g} 3333 \mathrm{~b}(\mathrm{i}) \cdot{ }^{*} \mathrm{Wq}\right)+\operatorname{sum}\left(\mathrm{g} 3333 \mathrm{a}(\mathrm{i}) .{ }^{*} \mathrm{Wq}\right)+\operatorname{sum}(\mathrm{g} 33\right.\right.$

$\left.\left.\left.\left.33 \mathrm{~b}(\mathrm{i}) *{ }^{*} \mathrm{Wq}\right)\right)\right)\right) /(8 * \mathrm{pi})$;

S2311

$\left(\left(\mathrm{L}(1,1) *\left(\operatorname{sum}\left(\mathrm{g} 2131 \mathrm{a}(\mathrm{i}) .{ }^{*} \mathrm{Wq}\right)+\operatorname{sum}\left(\mathrm{g} 2131 \mathrm{~b}(\mathrm{i}) .{ }^{*} \mathrm{Wq}\right)+\operatorname{sum}(\mathrm{g} 3121 \mathrm{a}(\mathrm{i}) . * \mathrm{Wq})+\operatorname{sum}(\mathrm{g} 3\right.\right.\right.$ $121 \mathrm{~b}(\mathrm{i}) \cdot * \mathrm{Wq})))$

$\left(\mathrm{L}(6,1) *\left(\operatorname{sum}\left(\mathrm{g} 2132 \mathrm{a}(\mathrm{i}) .{ }^{*} \mathrm{Wq}\right)+\operatorname{sum}\left(\mathrm{g} 2132 \mathrm{~b}(\mathrm{i}) .{ }^{*} \mathrm{Wq}\right)+\operatorname{sum}\left(\mathrm{g} 3122 \mathrm{a}(\mathrm{i}) .{ }^{*} \mathrm{Wq}\right)+\operatorname{sum}(\mathrm{g} 31\right.\right.$ $\left.\left.\left.22 \mathrm{~b}(\mathrm{i}){ }^{*} \mathrm{Wq}\right)\right)\right)$

$\left(\mathrm{L}(8,1) *\left(\operatorname{sum}\left(\mathrm{g} 2133 \mathrm{a}(\mathrm{i}) .{ }^{*} \mathrm{Wq}\right)+\operatorname{sum}\left(\mathrm{g} 2133 \mathrm{~b}(\mathrm{i}) .{ }^{*} \mathrm{Wq}\right)+\operatorname{sum}\left(\mathrm{g} 3123 \mathrm{a}(\mathrm{i}) .{ }^{*} \mathrm{Wq}\right)+\operatorname{sum}(\mathrm{g} 31\right.\right.$

$23 \mathrm{~b}(\mathrm{i}) . * \mathrm{Wq})))$

$\left(\mathrm{L}(9,1) *\left(\operatorname{sum}\left(\mathrm{g} 2231 \mathrm{a}(\mathrm{i}) .{ }^{*} \mathrm{Wq}\right)+\operatorname{sum}\left(\mathrm{g} 2231 \mathrm{~b}(\mathrm{i}) .{ }^{*} \mathrm{Wq}\right)+\operatorname{sum}\left(\mathrm{g} 3221 \mathrm{a}(\mathrm{i}) .{ }^{*} \mathrm{Wq}\right)+\operatorname{sum}(\mathrm{g} 32\right.\right.$

$\left.\left.\left.21 \mathrm{~b}(\mathrm{i}) \cdot{ }^{*} \mathrm{Wq}\right)\right)\right)$

$\left(\mathrm{L}(2,1) *\left(\operatorname{sum}\left(\mathrm{g} 2232 \mathrm{a}(\mathrm{i}) \cdot{ }^{*} \mathrm{Wq}\right)+\operatorname{sum}\left(\mathrm{g} 2232 \mathrm{~b}(\mathrm{i}) \cdot{ }^{*} \mathrm{Wq}\right)+\operatorname{sum}\left(\mathrm{g} 3222 \mathrm{a}(\mathrm{i}) \cdot{ }^{*} \mathrm{Wq}\right)+\operatorname{sum}(\mathrm{g} 32\right.\right.$

$22 \mathrm{~b}(\mathrm{i}) . * \mathrm{Wq})))$

$\left(\mathrm{L}(4,1) *\left(\operatorname{sum}\left(\mathrm{g} 2233 \mathrm{a}(\mathrm{i}) .{ }^{*} \mathrm{Wq}\right)+\operatorname{sum}\left(\mathrm{g} 2233 \mathrm{~b}(\mathrm{i}) \cdot{ }^{*} \mathrm{Wq}\right)+\operatorname{sum}\left(\mathrm{g} 3223 \mathrm{a}(\mathrm{i}) \cdot{ }^{*} \mathrm{Wq}\right)+\operatorname{sum}(\mathrm{g} 32\right.\right.$

$23 \mathrm{~b}(\mathrm{i}) . * \mathrm{Wq}))$

$\left(\mathrm{L}(5,1) *\left(\operatorname{sum}(\mathrm{g} 2331 \mathrm{a}(\mathrm{i}) . * \mathrm{Wq})+\operatorname{sum}\left(\mathrm{g} 2331 \mathrm{~b}(\mathrm{i}) \cdot{ }^{*} \mathrm{Wq}\right)+\operatorname{sum}\left(\mathrm{g} 3321 \mathrm{a}(\mathrm{i}) \cdot{ }^{*} \mathrm{Wq}\right)+\operatorname{sum}(\mathrm{g} 33\right.\right.$

$21 \mathrm{~b}(\mathrm{i}) \cdot * \mathrm{Wq})))$

$\left(\mathrm{L}(7,1) *\left(\operatorname{sum}\left(\mathrm{g} 2332 \mathrm{a}(\mathrm{i}) .{ }^{*} \mathrm{Wq}\right)+\operatorname{sum}\left(\mathrm{g} 2332 \mathrm{~b}(\mathrm{i}) \cdot{ }^{*} \mathrm{Wq}\right)+\operatorname{sum}\left(\mathrm{g} 3322 \mathrm{a}(\mathrm{i}) .{ }^{*} \mathrm{Wq}\right)+\operatorname{sum}(\mathrm{g} 33\right.\right.$

$22 \mathrm{~b}(\mathrm{i}) . * \mathrm{Wq})))$

$\left(\mathrm{L}(3,1) *\left(\operatorname{sum}\left(\mathrm{g} 2333 \mathrm{a}(\mathrm{i}) .{ }^{*} \mathrm{Wq}\right)+\operatorname{sum}\left(\mathrm{g} 2333 \mathrm{~b}(\mathrm{i}) \cdot{ }^{*} \mathrm{Wq}\right)+\operatorname{sum}\left(\mathrm{g} 3323 \mathrm{a}(\mathrm{i}) .{ }^{*} \mathrm{Wq}\right)+\operatorname{sum}(\mathrm{g} 33\right.\right.$

$23 \mathrm{~b}(\mathrm{i}) *$ *Wq $)))) /(8 * \mathrm{pi})$

$\mathrm{S} 2322$

$\left(\left(\mathrm{L}(1,2) *\left(\operatorname{sum}(\mathrm{g} 2131 \mathrm{a}(\mathrm{i}) . * \mathrm{Wq})+\operatorname{sum}\left(\mathrm{g} 2131 \mathrm{~b}(\mathrm{i}) \cdot{ }^{*} \mathrm{Wq}\right)+\operatorname{sum}(\mathrm{g} 3121 \mathrm{a}(\mathrm{i}) . * \mathrm{Wq})+\operatorname{sum}(\mathrm{g} 3\right.\right.\right.$ $\left.\left.\left.121 \mathrm{~b}(\mathrm{i}) \cdot{ }^{*} \mathrm{Wq}\right)\right)\right)$

$\left(\mathrm{L}(6,2) *\left(\operatorname{sum}\left(\mathrm{g} 2132 \mathrm{a}(\mathrm{i}) .{ }^{*} \mathrm{Wq}\right)+\operatorname{sum}\left(\mathrm{g} 2132 \mathrm{~b}(\mathrm{i}) \cdot{ }^{*} \mathrm{Wq}\right)+\operatorname{sum}(\mathrm{g} 3122 \mathrm{a}(\mathrm{i}) . * \mathrm{Wq})+\operatorname{sum}(\mathrm{g} 31\right.\right.$ 
$22 \mathrm{~b}(\mathrm{i}) \cdot * \mathrm{Wq})))$

$\left(\mathrm{L}(8,2) *\left(\operatorname{sum}(\mathrm{g} 2133 \mathrm{a}(\mathrm{i}) . * \mathrm{Wq})+\operatorname{sum}\left(\mathrm{g} 2133 \mathrm{~b}(\mathrm{i}) \cdot{ }^{*} \mathrm{Wq}\right)+\operatorname{sum}\left(\mathrm{g} 3123 \mathrm{a}(\mathrm{i}) \cdot{ }^{*} \mathrm{Wq}\right)+\operatorname{sum}(\mathrm{g} 31\right.\right.$

$\left.\left.\left.23 \mathrm{~b}(\mathrm{i}) *{ }^{*} \mathrm{Wq}\right)\right)\right)$

$\left(\mathrm{L}(9,2) *\left(\operatorname{sum}\left(\mathrm{g} 2231 \mathrm{a}(\mathrm{i}) .{ }^{*} \mathrm{Wq}\right)+\operatorname{sum}\left(\mathrm{g} 2231 \mathrm{~b}(\mathrm{i}) .{ }^{*} \mathrm{Wq}\right)+\operatorname{sum}\left(\mathrm{g} 3221 \mathrm{a}(\mathrm{i}) \cdot{ }^{*} \mathrm{Wq}\right)+\operatorname{sum}(\mathrm{g} 32\right.\right.$

$21 \mathrm{~b}(\mathrm{i}) . * \mathrm{Wq})))$

$\left(\mathrm{L}(2,2) *\left(\operatorname{sum}(\mathrm{g} 2232 \mathrm{a}(\mathrm{i}) . * \mathrm{Wq})+\operatorname{sum}\left(\mathrm{g} 2232 \mathrm{~b}(\mathrm{i}) \cdot{ }^{*} \mathrm{Wq}\right)+\operatorname{sum}\left(\mathrm{g} 3222 \mathrm{a}(\mathrm{i}) \cdot{ }^{*} \mathrm{Wq}\right)+\operatorname{sum}(\mathrm{g} 32\right.\right.$

$22 \mathrm{~b}(\mathrm{i}) . * \mathrm{Wq})))$

$\left(\mathrm{L}(4,2) *\left(\operatorname{sum}\left(\mathrm{g} 2233 \mathrm{a}(\mathrm{i}) .{ }^{*} \mathrm{Wq}\right)+\operatorname{sum}\left(\mathrm{g} 2233 \mathrm{~b}(\mathrm{i}) \cdot{ }^{*} \mathrm{Wq}\right)+\operatorname{sum}\left(\mathrm{g} 3223 \mathrm{a}(\mathrm{i}) .{ }^{*} \mathrm{Wq}\right)+\operatorname{sum}(\mathrm{g} 32\right.\right.$ $\left.\left.23 \mathrm{~b}(\mathrm{i}) \cdot{ }^{*} \mathrm{Wq}\right)\right)$

$\left(\mathrm{L}(5,2) *\left(\operatorname{sum}\left(\mathrm{g} 2331 \mathrm{a}(\mathrm{i}) .{ }^{*} \mathrm{Wq}\right)+\operatorname{sum}\left(\mathrm{g} 2331 \mathrm{~b}(\mathrm{i}) .{ }^{*} \mathrm{Wq}\right)+\operatorname{sum}\left(\mathrm{g} 3321 \mathrm{a}(\mathrm{i}) .{ }^{*} \mathrm{Wq}\right)+\operatorname{sum}(\mathrm{g} 33\right.\right.$

$21 \mathrm{~b}(\mathrm{i}) . * \mathrm{Wq})))$

$\left(\mathrm{L}(7,2) *\left(\operatorname{sum}\left(\mathrm{g} 2332 \mathrm{a}(\mathrm{i}) .{ }^{*} \mathrm{Wq}\right)+\operatorname{sum}\left(\mathrm{g} 2332 \mathrm{~b}(\mathrm{i}) .{ }^{*} \mathrm{Wq}\right)+\operatorname{sum}(\mathrm{g} 3322 \mathrm{a}(\mathrm{i}) . * \mathrm{Wq})+\operatorname{sum}(\mathrm{g} 33\right.\right.$

$22 \mathrm{~b}(\mathrm{i}) \cdot * \mathrm{Wq}))$

$\left(\mathrm{L}(3,2) *\left(\operatorname{sum}\left(\mathrm{g} 2333 \mathrm{a}(\mathrm{i}) .{ }^{*} \mathrm{Wq}\right)+\operatorname{sum}\left(\mathrm{g} 2333 \mathrm{~b}(\mathrm{i}) \cdot{ }^{*} \mathrm{Wq}\right)+\operatorname{sum}\left(\mathrm{g} 3323 \mathrm{a}(\mathrm{i}) .{ }^{*} \mathrm{Wq}\right)+\operatorname{sum}(\mathrm{g} 33\right.\right.$

$\left.\left.\left.\left.23 \mathrm{~b}(\mathrm{i}) \cdot{ }^{*} \mathrm{Wq}\right)\right)\right)\right) /(8 * \mathrm{pi})$;

$\mathrm{S} 2333$

$\left(\left(\mathrm{L}(1,3) *\left(\operatorname{sum}\left(\mathrm{g} 2131 \mathrm{a}(\mathrm{i}) .{ }^{*} \mathrm{Wq}\right)+\operatorname{sum}\left(\mathrm{g} 2131 \mathrm{~b}(\mathrm{i}) .{ }^{*} \mathrm{Wq}\right)+\operatorname{sum}(\mathrm{g} 3121 \mathrm{a}(\mathrm{i}) . * \mathrm{Wq})+\operatorname{sum}(\mathrm{g} 3\right.\right.\right.$ $121 \mathrm{~b}(\mathrm{i}) * \mathrm{Wq})))$

$\left(\mathrm{L}(6,3) *\left(\operatorname{sum}\left(\mathrm{g} 2132 \mathrm{a}(\mathrm{i}) .{ }^{*} \mathrm{Wq}\right)+\operatorname{sum}\left(\mathrm{g} 2132 \mathrm{~b}(\mathrm{i}) \cdot{ }^{*} \mathrm{Wq}\right)+\operatorname{sum}\left(\mathrm{g} 3122 \mathrm{a}(\mathrm{i}) .{ }^{*} \mathrm{Wq}\right)+\operatorname{sum}(\mathrm{g} 31\right.\right.$ $22 \mathrm{~b}(\mathrm{i}) . * \mathrm{Wq})))$

$\left(\mathrm{L}(8,3) *\left(\operatorname{sum}\left(\mathrm{g} 2133 \mathrm{a}(\mathrm{i}) .{ }^{*} \mathrm{Wq}\right)+\operatorname{sum}\left(\mathrm{g} 2133 \mathrm{~b}(\mathrm{i}) \cdot{ }^{*} \mathrm{Wq}\right)+\operatorname{sum}\left(\mathrm{g} 3123 \mathrm{a}(\mathrm{i}) .{ }^{*} \mathrm{Wq}\right)+\operatorname{sum}(\mathrm{g} 31\right.\right.$

$23 \mathrm{~b}(\mathrm{i}) . * \mathrm{Wq})))$

$\left(\mathrm{L}(9,3) *\left(\operatorname{sum}\left(\mathrm{g} 2231 \mathrm{a}(\mathrm{i}) .{ }^{*} \mathrm{Wq}\right)+\operatorname{sum}\left(\mathrm{g} 2231 \mathrm{~b}(\mathrm{i}) .{ }^{*} \mathrm{Wq}\right)+\operatorname{sum}\left(\mathrm{g} 3221 \mathrm{a}(\mathrm{i}) \cdot{ }^{*} \mathrm{Wq}\right)+\operatorname{sum}(\mathrm{g} 32\right.\right.$

$21 \mathrm{~b}(\mathrm{i}) . * \mathrm{Wq})))$

$\left(\mathrm{L}(2,3) *\left(\operatorname{sum}\left(\mathrm{g} 2232 \mathrm{a}(\mathrm{i}) \cdot{ }^{*} \mathrm{Wq}\right)+\operatorname{sum}\left(\mathrm{g} 2232 \mathrm{~b}(\mathrm{i}) \cdot{ }^{*} \mathrm{Wq}\right)+\operatorname{sum}\left(\mathrm{g} 3222 \mathrm{a}(\mathrm{i}) \cdot{ }^{*} \mathrm{Wq}\right)+\operatorname{sum}(\mathrm{g} 32\right.\right.$

$22 \mathrm{~b}(\mathrm{i}) \cdot * \mathrm{Wq}))$

$\left(\mathrm{L}(4,3) *\left(\operatorname{sum}\left(\mathrm{g} 2233 \mathrm{a}(\mathrm{i}) .{ }^{*} \mathrm{Wq}\right)+\operatorname{sum}\left(\mathrm{g} 2233 \mathrm{~b}(\mathrm{i}) .{ }^{*} \mathrm{Wq}\right)+\operatorname{sum}\left(\mathrm{g} 3223 \mathrm{a}(\mathrm{i}) .{ }^{*} \mathrm{Wq}\right)+\operatorname{sum}(\mathrm{g} 32\right.\right.$

$23 \mathrm{~b}(\mathrm{i}) . * \mathrm{Wq}))$

$\left(\mathrm{L}(5,3) *\left(\operatorname{sum}\left(\mathrm{g} 2331 \mathrm{a}(\mathrm{i}) .{ }^{*} \mathrm{Wq}\right)+\operatorname{sum}\left(\mathrm{g} 2331 \mathrm{~b}(\mathrm{i}) \cdot{ }^{*} \mathrm{Wq}\right)+\operatorname{sum}\left(\mathrm{g} 3321 \mathrm{a}(\mathrm{i}) .{ }^{*} \mathrm{Wq}\right)+\operatorname{sum}(\mathrm{g} 33\right.\right.$

$21 \mathrm{~b}(\mathrm{i}) . * \mathrm{Wq})))$

$\left(\mathrm{L}(7,3) *\left(\operatorname{sum}\left(\mathrm{g} 2332 \mathrm{a}(\mathrm{i}) .{ }^{*} \mathrm{Wq}\right)+\operatorname{sum}\left(\mathrm{g} 2332 \mathrm{~b}(\mathrm{i}) .{ }^{*} \mathrm{Wq}\right)+\operatorname{sum}(\mathrm{g} 3322 \mathrm{a}(\mathrm{i}) . * \mathrm{Wq})+\operatorname{sum}(\mathrm{g} 33\right.\right.$

$\left.\left.22 \mathrm{~b}(\mathrm{i}) \cdot{ }^{*} \mathrm{Wq}\right)\right)$

$\left(\mathrm{L}(3,3) *\left(\operatorname{sum}\left(\mathrm{g} 2333 \mathrm{a}(\mathrm{i}) .{ }^{*} \mathrm{Wq}\right)+\operatorname{sum}\left(\mathrm{g} 2333 \mathrm{~b}(\mathrm{i}) \cdot{ }^{*} \mathrm{Wq}\right)+\operatorname{sum}\left(\mathrm{g} 3323 \mathrm{a}(\mathrm{i}) .{ }^{*} \mathrm{Wq}\right)+\operatorname{sum}(\mathrm{g} 33\right.\right.$

$\left.\left.\left.\left.23 \mathrm{~b}(\mathrm{i}) \cdot{ }^{*} \mathrm{Wq}\right)\right)\right)\right) /(8 * \mathrm{pi})$;

$\mathrm{S} 2323$

$\left(\left(\mathrm{L}(1,4) *\left(\operatorname{sum}(\mathrm{g} 2131 \mathrm{a}(\mathrm{i}) . * \mathrm{Wq})+\operatorname{sum}(\mathrm{g} 2131 \mathrm{~b}(\mathrm{i}) . * \mathrm{Wq})+\operatorname{sum}\left(\mathrm{g} 3121 \mathrm{a}(\mathrm{i}) .{ }^{*} \mathrm{Wq}\right)+\operatorname{sum}(\mathrm{g} 3\right.\right.\right.$ $121 \mathrm{~b}(\mathrm{i}) \cdot * \mathrm{Wq})))$

$\left(\mathrm{L}(6,4) *\left(\operatorname{sum}\left(\mathrm{g} 2132 \mathrm{a}(\mathrm{i}) .{ }^{*} \mathrm{Wq}\right)+\operatorname{sum}\left(\mathrm{g} 2132 \mathrm{~b}(\mathrm{i}) \cdot{ }^{*} \mathrm{Wq}\right)+\operatorname{sum}\left(\mathrm{g} 3122 \mathrm{a}(\mathrm{i}) .{ }^{*} \mathrm{Wq}\right)+\operatorname{sum}(\mathrm{g} 31\right.\right.$ $\left.\left.\left.22 \mathrm{~b}(\mathrm{i}){ }^{*} \mathrm{Wq}\right)\right)\right)$

$\left(\mathrm{L}(8,4) *\left(\operatorname{sum}\left(\mathrm{g} 2133 \mathrm{a}(\mathrm{i}) .{ }^{*} \mathrm{Wq}\right)+\operatorname{sum}\left(\mathrm{g} 2133 \mathrm{~b}(\mathrm{i}) .{ }^{*} \mathrm{Wq}\right)+\operatorname{sum}\left(\mathrm{g} 3123 \mathrm{a}(\mathrm{i}) .{ }^{*} \mathrm{Wq}\right)+\operatorname{sum}(\mathrm{g} 31\right.\right.$

$23 \mathrm{~b}(\mathrm{i}) \cdot * \mathrm{Wq})))$

$\left(\mathrm{L}(9,4) *\left(\operatorname{sum}(\mathrm{g} 2231 \mathrm{a}(\mathrm{i}) . * \mathrm{Wq})+\operatorname{sum}\left(\mathrm{g} 2231 \mathrm{~b}(\mathrm{i}) \cdot{ }^{*} \mathrm{Wq}\right)+\operatorname{sum}\left(\mathrm{g} 3221 \mathrm{a}(\mathrm{i}) \cdot{ }^{*} \mathrm{Wq}\right)+\operatorname{sum}(\mathrm{g} 32\right.\right.$

$21 \mathrm{~b}(\mathrm{i}) . * \mathrm{Wq})))$ 
$\left(\mathrm{L}(2,4) *\left(\operatorname{sum}\left(\mathrm{g} 2232 \mathrm{a}(\mathrm{i}) \cdot{ }^{*} \mathrm{Wq}\right)+\operatorname{sum}\left(\mathrm{g} 2232 \mathrm{~b}(\mathrm{i}) \cdot{ }^{*} \mathrm{Wq}\right)+\operatorname{sum}\left(\mathrm{g} 3222 \mathrm{a}(\mathrm{i}) \cdot{ }^{*} \mathrm{Wq}\right)+\operatorname{sum}(\mathrm{g} 32\right.\right.$ $22 \mathrm{~b}(\mathrm{i}) . * \mathrm{Wq})))$ $\left(\mathrm{L}(4,4) *\left(\operatorname{sum}(\mathrm{g} 2233 \mathrm{a}(\mathrm{i}) . * \mathrm{Wq})+\operatorname{sum}\left(\mathrm{g} 2233 \mathrm{~b}(\mathrm{i}) .{ }^{*} \mathrm{Wq}\right)+\operatorname{sum}(\mathrm{g} 3223 \mathrm{a}(\mathrm{i}) . * \mathrm{Wq})+\operatorname{sum}(\mathrm{g} 32\right.\right.$ $23 \mathrm{~b}(\mathrm{i}) . * \mathrm{Wq})))$ $\left(\mathrm{L}(5,4) *\left(\operatorname{sum}\left(\mathrm{g} 2331 \mathrm{a}(\mathrm{i}) .{ }^{*} \mathrm{Wq}\right)+\operatorname{sum}\left(\mathrm{g} 2331 \mathrm{~b}(\mathrm{i}) .{ }^{*} \mathrm{Wq}\right)+\operatorname{sum}\left(\mathrm{g} 3321 \mathrm{a}(\mathrm{i}) .{ }^{*} \mathrm{Wq}\right)+\operatorname{sum}(\mathrm{g} 33\right.\right.$ $21 \mathrm{~b}(\mathrm{i}) . * \mathrm{Wq})))$ $\left(\mathrm{L}(7,4) *\left(\operatorname{sum}\left(\mathrm{g} 2332 \mathrm{a}(\mathrm{i}) .{ }^{*} \mathrm{Wq}\right)+\operatorname{sum}\left(\mathrm{g} 2332 \mathrm{~b}(\mathrm{i}) \cdot{ }^{*} \mathrm{Wq}\right)+\operatorname{sum}\left(\mathrm{g} 3322 \mathrm{a}(\mathrm{i}) .{ }^{*} \mathrm{Wq}\right)+\operatorname{sum}(\mathrm{g} 33\right.\right.$ $22 \mathrm{~b}(\mathrm{i}) . * \mathrm{Wq})))$ $\left(\mathrm{L}(3,4) *\left(\operatorname{sum}\left(\mathrm{g} 2333 \mathrm{a}(\mathrm{i}) .{ }^{*} \mathrm{Wq}\right)+\operatorname{sum}\left(\mathrm{g} 2333 \mathrm{~b}(\mathrm{i}) .{ }^{*} \mathrm{Wq}\right)+\operatorname{sum}\left(\mathrm{g} 3323 \mathrm{a}(\mathrm{i}) .{ }^{*} \mathrm{Wq}\right)+\operatorname{sum}(\mathrm{g} 33\right.\right.$ $\left.\left.\left.\left.23 \mathrm{~b}(\mathrm{i}){ }^{*} \mathrm{Wq}\right)\right)\right)\right) /(8 * \mathrm{pi})$;

$\mathrm{S} 2331$

$\left(\left(\mathrm{L}(1,5) *\left(\operatorname{sum}\left(\mathrm{g} 2131 \mathrm{a}(\mathrm{i}) .{ }^{*} \mathrm{Wq}\right)+\operatorname{sum}\left(\mathrm{g} 2131 \mathrm{~b}(\mathrm{i}) .{ }^{*} \mathrm{Wq}\right)+\operatorname{sum}\left(\mathrm{g} 3121 \mathrm{a}(\mathrm{i}) .{ }^{*} \mathrm{Wq}\right)+\operatorname{sum}(\mathrm{g} 3\right.\right.\right.$ $\left.\left.\left.121 \mathrm{~b}(\mathrm{i}) \cdot{ }^{*} \mathrm{Wq}\right)\right)\right)$ $\left(\mathrm{L}(6,5) *\left(\operatorname{sum}\left(\mathrm{g} 2132 \mathrm{a}(\mathrm{i}) .{ }^{*} \mathrm{Wq}\right)+\operatorname{sum}\left(\mathrm{g} 2132 \mathrm{~b}(\mathrm{i}) \cdot{ }^{*} \mathrm{Wq}\right)+\operatorname{sum}\left(\mathrm{g} 3122 \mathrm{a}(\mathrm{i}) .{ }^{*} \mathrm{Wq}\right)+\operatorname{sum}(\mathrm{g} 31\right.\right.$ $22 \mathrm{~b}(\mathrm{i}) . * \mathrm{Wq}))$ ) $\left(\mathrm{L}(8,5) *\left(\operatorname{sum}\left(\mathrm{g} 2133 \mathrm{a}(\mathrm{i}) .{ }^{*} \mathrm{Wq}\right)+\operatorname{sum}\left(\mathrm{g} 2133 \mathrm{~b}(\mathrm{i}) .{ }^{*} \mathrm{Wq}\right)+\operatorname{sum}(\mathrm{g} 3123 \mathrm{a}(\mathrm{i}) . * \mathrm{Wq})+\operatorname{sum}(\mathrm{g} 31\right.\right.$ $23 \mathrm{~b}(\mathrm{i}) . * \mathrm{Wq})))$ $\left(\mathrm{L}(9,5) *\left(\operatorname{sum}\left(\mathrm{g} 2231 \mathrm{a}(\mathrm{i}) .{ }^{*} \mathrm{Wq}\right)+\operatorname{sum}\left(\mathrm{g} 2231 \mathrm{~b}(\mathrm{i}) .{ }^{*} \mathrm{Wq}\right)+\operatorname{sum}\left(\mathrm{g} 3221 \mathrm{a}(\mathrm{i}) \cdot{ }^{*} \mathrm{Wq}\right)+\operatorname{sum}(\mathrm{g} 32\right.\right.$ $21 \mathrm{~b}(\mathrm{i}) . * \mathrm{Wq})))$ $\left(\mathrm{L}(2,5) *\left(\operatorname{sum}\left(\mathrm{g} 2232 \mathrm{a}(\mathrm{i}) .{ }^{*} \mathrm{Wq}\right)+\operatorname{sum}\left(\mathrm{g} 2232 \mathrm{~b}(\mathrm{i}) \cdot{ }^{*} \mathrm{Wq}\right)+\operatorname{sum}\left(\mathrm{g} 3222 \mathrm{a}(\mathrm{i}) \cdot{ }^{*} \mathrm{Wq}\right)+\operatorname{sum}(\mathrm{g} 32\right.\right.$ $22 \mathrm{~b}(\mathrm{i}) . * \mathrm{Wq})))$ $\left(\mathrm{L}(4,5) *\left(\operatorname{sum}(\mathrm{g} 2233 \mathrm{a}(\mathrm{i}) . * \mathrm{Wq})+\operatorname{sum}\left(\mathrm{g} 2233 \mathrm{~b}(\mathrm{i}) \cdot{ }^{*} \mathrm{Wq}\right)+\operatorname{sum}\left(\mathrm{g} 3223 \mathrm{a}(\mathrm{i}) \cdot{ }^{*} \mathrm{Wq}\right)+\operatorname{sum}(\mathrm{g} 32\right.\right.$ $23 \mathrm{~b}(\mathrm{i}) . * \mathrm{Wq})))$ $\left(\mathrm{L}(5,5) *\left(\operatorname{sum}(\mathrm{g} 2331 \mathrm{a}(\mathrm{i}) . * \mathrm{Wq})+\operatorname{sum}\left(\mathrm{g} 2331 \mathrm{~b}(\mathrm{i}) \cdot{ }^{*} \mathrm{Wq}\right)+\operatorname{sum}\left(\mathrm{g} 3321 \mathrm{a}(\mathrm{i}) .{ }^{*} \mathrm{Wq}\right)+\operatorname{sum}(\mathrm{g} 33\right.\right.$ $21 \mathrm{~b}(\mathrm{i}) . * \mathrm{Wq})))$ $\left(\mathrm{L}(7,5) *\left(\operatorname{sum}\left(\mathrm{g} 2332 \mathrm{a}(\mathrm{i}) .{ }^{*} \mathrm{Wq}\right)+\operatorname{sum}\left(\mathrm{g} 2332 \mathrm{~b}(\mathrm{i}) \cdot{ }^{*} \mathrm{Wq}\right)+\operatorname{sum}\left(\mathrm{g} 3322 \mathrm{a}(\mathrm{i}) \cdot{ }^{*} \mathrm{Wq}\right)+\operatorname{sum}(\mathrm{g} 33\right.\right.$ $22 \mathrm{~b}(\mathrm{i}) . * \mathrm{Wq})))$ $\left(\mathrm{L}(3,5) *\left(\operatorname{sum}\left(\mathrm{g} 2333 \mathrm{a}(\mathrm{i}) .{ }^{*} \mathrm{Wq}\right)+\operatorname{sum}\left(\mathrm{g} 2333 \mathrm{~b}(\mathrm{i}) .{ }^{*} \mathrm{Wq}\right)+\operatorname{sum}\left(\mathrm{g} 3323 \mathrm{a}(\mathrm{i}) .{ }^{*} \mathrm{Wq}\right)+\operatorname{sum}(\mathrm{g} 33\right.\right.$ $23 \mathrm{~b}(\mathrm{i}) . * \mathrm{Wq})))) /(8 * \mathrm{pi})$;

$\mathrm{S} 2312$

$\left(\left(\mathrm{L}(1,6) *\left(\operatorname{sum}\left(\mathrm{g} 2131 \mathrm{a}(\mathrm{i}) .{ }^{*} \mathrm{Wq}\right)+\operatorname{sum}\left(\mathrm{g} 2131 \mathrm{~b}(\mathrm{i}) .{ }^{*} \mathrm{Wq}\right)+\operatorname{sum}\left(\mathrm{g} 3121 \mathrm{a}(\mathrm{i}) .{ }^{*} \mathrm{Wq}\right)+\operatorname{sum}(\mathrm{g} 3\right.\right.\right.$ $121 \mathrm{~b}(\mathrm{i}) * \mathrm{Wq}))$ $\left(\mathrm{L}(6,6) *\left(\operatorname{sum}\left(\mathrm{g} 2132 \mathrm{a}(\mathrm{i}) .{ }^{*} \mathrm{Wq}\right)+\operatorname{sum}\left(\mathrm{g} 2132 \mathrm{~b}(\mathrm{i}) .{ }^{*} \mathrm{Wq}\right)+\operatorname{sum}(\mathrm{g} 3122 \mathrm{a}(\mathrm{i}) . * \mathrm{Wq})+\operatorname{sum}(\mathrm{g} 31\right.\right.$ $22 \mathrm{~b}(\mathrm{i}) . * \mathrm{Wq})))$ $\left(\mathrm{L}(8,6) *\left(\operatorname{sum}(\mathrm{g} 2133 \mathrm{a}(\mathrm{i}) . * \mathrm{Wq})+\operatorname{sum}\left(\mathrm{g} 2133 \mathrm{~b}(\mathrm{i}) \cdot{ }^{*} \mathrm{Wq}\right)+\operatorname{sum}(\mathrm{g} 3123 \mathrm{a}(\mathrm{i}) . * \mathrm{Wq})+\operatorname{sum}(\mathrm{g} 31\right.\right.$ $23 \mathrm{~b}(\mathrm{i}) . * \mathrm{Wq})))$ $\left(\mathrm{L}(9,6) *\left(\operatorname{sum}\left(\mathrm{g} 2231 \mathrm{a}(\mathrm{i}) .{ }^{*} \mathrm{Wq}\right)+\operatorname{sum}\left(\mathrm{g} 2231 \mathrm{~b}(\mathrm{i}) \cdot{ }^{*} \mathrm{Wq}\right)+\operatorname{sum}\left(\mathrm{g} 3221 \mathrm{a}(\mathrm{i}) \cdot{ }^{*} \mathrm{Wq}\right)+\operatorname{sum}(\mathrm{g} 32\right.\right.$ $21 \mathrm{~b}(\mathrm{i}) . * \mathrm{Wq})))$ $\left(\mathrm{L}(2,6) *\left(\operatorname{sum}\left(\mathrm{g} 2232 \mathrm{a}(\mathrm{i}) .{ }^{*} \mathrm{Wq}\right)+\operatorname{sum}\left(\mathrm{g} 2232 \mathrm{~b}(\mathrm{i}) .{ }^{*} \mathrm{Wq}\right)+\operatorname{sum}\left(\mathrm{g} 3222 \mathrm{a}(\mathrm{i}) .{ }^{*} \mathrm{Wq}\right)+\operatorname{sum}(\mathrm{g} 32\right.\right.$ $22 \mathrm{~b}(\mathrm{i}) . * \mathrm{Wq})))$ $\left(\mathrm{L}(4,6) *\left(\operatorname{sum}(\mathrm{g} 2233 \mathrm{a}(\mathrm{i}) . * \mathrm{Wq})+\operatorname{sum}\left(\mathrm{g} 2233 \mathrm{~b}(\mathrm{i}) \cdot{ }^{*} \mathrm{Wq}\right)+\operatorname{sum}\left(\mathrm{g} 3223 \mathrm{a}(\mathrm{i}) \cdot{ }^{*} \mathrm{Wq}\right)+\operatorname{sum}(\mathrm{g} 32\right.\right.$ $23 \mathrm{~b}(\mathrm{i}) . * \mathrm{Wq})))$ $\left(\mathrm{L}(5,6) *\left(\operatorname{sum}\left(\mathrm{g} 2331 \mathrm{a}(\mathrm{i}) .{ }^{*} \mathrm{Wq}\right)+\operatorname{sum}\left(\mathrm{g} 2331 \mathrm{~b}(\mathrm{i}) \cdot{ }^{*} \mathrm{Wq}\right)+\operatorname{sum}\left(\mathrm{g} 3321 \mathrm{a}(\mathrm{i}) .{ }^{*} \mathrm{Wq}\right)+\operatorname{sum}(\mathrm{g} 33\right.\right.$ 
$21 \mathrm{~b}(\mathrm{i}) . * \mathrm{Wq})))$

$\left(\mathrm{L}(7,6) *\left(\operatorname{sum}\left(\mathrm{g} 2332 \mathrm{a}(\mathrm{i}) \cdot{ }^{*} \mathrm{Wq}\right)+\operatorname{sum}\left(\mathrm{g} 2332 \mathrm{~b}(\mathrm{i}) \cdot{ }^{*} \mathrm{Wq}\right)+\operatorname{sum}\left(\mathrm{g} 3322 \mathrm{a}(\mathrm{i}) .{ }^{*} \mathrm{Wq}\right)+\operatorname{sum}(\mathrm{g} 33\right.\right.$

$22 \mathrm{~b}(\mathrm{i}) . * \mathrm{Wq})))$

$\left(\mathrm{L}(3,6) *\left(\operatorname{sum}\left(\mathrm{g} 2333 \mathrm{a}(\mathrm{i}) .{ }^{*} \mathrm{Wq}\right)+\operatorname{sum}\left(\mathrm{g} 2333 \mathrm{~b}(\mathrm{i}) \cdot{ }^{*} \mathrm{Wq}\right)+\operatorname{sum}\left(\mathrm{g} 3323 \mathrm{a}(\mathrm{i}) .{ }^{*} \mathrm{Wq}\right)+\operatorname{sum}(\mathrm{g} 33\right.\right.$

$23 \mathrm{~b}(\mathrm{i}) *$ (Wq) $))) /(8 * \mathrm{pi})$;

$\mathrm{S} 2332$

$\left(\left(\mathrm{L}(1,7) *\left(\operatorname{sum}\left(\mathrm{g} 2131 \mathrm{a}(\mathrm{i}) .{ }^{*} \mathrm{Wq}\right)+\operatorname{sum}\left(\mathrm{g} 2131 \mathrm{~b}(\mathrm{i}) .{ }^{*} \mathrm{Wq}\right)+\operatorname{sum}\left(\mathrm{g} 3121 \mathrm{a}(\mathrm{i}) .{ }^{*} \mathrm{Wq}\right)+\operatorname{sum}(\mathrm{g} 3\right.\right.\right.$ $\left.\left.\left.121 \mathrm{~b}(\mathrm{i}) \cdot{ }^{*} \mathrm{Wq}\right)\right)\right)$

$\left(\mathrm{L}(6,7) *\left(\operatorname{sum}\left(\mathrm{g} 2132 \mathrm{a}(\mathrm{i}) .{ }^{*} \mathrm{Wq}\right)+\operatorname{sum}\left(\mathrm{g} 2132 \mathrm{~b}(\mathrm{i}) .{ }^{*} \mathrm{Wq}\right)+\operatorname{sum}\left(\mathrm{g} 3122 \mathrm{a}(\mathrm{i}) .{ }^{*} \mathrm{Wq}\right)+\operatorname{sum}(\mathrm{g} 31\right.\right.$ $22 \mathrm{~b}(\mathrm{i}) . * \mathrm{Wq}))$ )

$\left(\mathrm{L}(8,7) *\left(\operatorname{sum}\left(\mathrm{g} 2133 \mathrm{a}(\mathrm{i}) .{ }^{*} \mathrm{Wq}\right)+\operatorname{sum}(\mathrm{g} 2133 \mathrm{~b}(\mathrm{i}) . * \mathrm{Wq})+\operatorname{sum}\left(\mathrm{g} 3123 \mathrm{a}(\mathrm{i}) .{ }^{*} \mathrm{Wq}\right)+\operatorname{sum}(\mathrm{g} 31\right.\right.$ 23b(i).*Wq)))

$\left(\mathrm{L}(9,7) *\left(\operatorname{sum}\left(\mathrm{g} 2231 \mathrm{a}(\mathrm{i}) .{ }^{*} \mathrm{Wq}\right)+\operatorname{sum}\left(\mathrm{g} 2231 \mathrm{~b}(\mathrm{i}) .{ }^{*} \mathrm{Wq}\right)+\operatorname{sum}\left(\mathrm{g} 3221 \mathrm{a}(\mathrm{i}) .{ }^{*} \mathrm{Wq}\right)+\operatorname{sum}(\mathrm{g} 32\right.\right.$ $21 \mathrm{~b}(\mathrm{i}) * \mathrm{Wq}))$

$\left(\mathrm{L}(2,7) *\left(\operatorname{sum}\left(\mathrm{g} 2232 \mathrm{a}(\mathrm{i}) .{ }^{*} \mathrm{Wq}\right)+\operatorname{sum}(\mathrm{g} 2232 \mathrm{~b}(\mathrm{i}) . * \mathrm{Wq})+\operatorname{sum}\left(\mathrm{g} 3222 \mathrm{a}(\mathrm{i}) .{ }^{*} \mathrm{Wq}\right)+\operatorname{sum}(\mathrm{g} 32\right.\right.$ $\left.\left.\left.22 \mathrm{~b}(\mathrm{i}) *{ }^{*} \mathrm{Wq}\right)\right)\right)$

$\left(\mathrm{L}(4,7) *\left(\operatorname{sum}\left(\mathrm{g} 2233 \mathrm{a}(\mathrm{i}) .{ }^{*} \mathrm{Wq}\right)+\operatorname{sum}(\mathrm{g} 2233 \mathrm{~b}(\mathrm{i}) . * \mathrm{Wq})+\operatorname{sum}\left(\mathrm{g} 3223 \mathrm{a}(\mathrm{i}) .{ }^{*} \mathrm{Wq}\right)+\operatorname{sum}(\mathrm{g} 32\right.\right.$ 23b(i).*Wq)))

$\left(\mathrm{L}(5,7) *\left(\operatorname{sum}\left(\mathrm{g} 2331 \mathrm{a}(\mathrm{i}) .{ }^{*} \mathrm{Wq}\right)+\operatorname{sum}\left(\mathrm{g} 2331 \mathrm{~b}(\mathrm{i}) \cdot{ }^{*} \mathrm{Wq}\right)+\operatorname{sum}\left(\mathrm{g} 3321 \mathrm{a}(\mathrm{i}) .{ }^{*} \mathrm{Wq}\right)+\operatorname{sum}(\mathrm{g} 33\right.\right.$ $21 \mathrm{~b}(\mathrm{i}) . * \mathrm{Wq})))$

$\left(\mathrm{L}(7,7) *\left(\operatorname{sum}\left(\mathrm{g} 2332 \mathrm{a}(\mathrm{i}) .{ }^{*} \mathrm{Wq}\right)+\operatorname{sum}\left(\mathrm{g} 2332 \mathrm{~b}(\mathrm{i}) .{ }^{*} \mathrm{Wq}\right)+\operatorname{sum}\left(\mathrm{g} 3322 \mathrm{a}(\mathrm{i}) .{ }^{*} \mathrm{Wq}\right)+\operatorname{sum}(\mathrm{g} 33\right.\right.$ $22 \mathrm{~b}(\mathrm{i}) . * \mathrm{Wq})))$

$\left(\mathrm{L}(3,7) *\left(\operatorname{sum}\left(\mathrm{g} 2333 \mathrm{a}(\mathrm{i}) .{ }^{*} \mathrm{Wq}\right)+\operatorname{sum}\left(\mathrm{g} 2333 \mathrm{~b}(\mathrm{i}) \cdot{ }^{*} \mathrm{Wq}\right)+\operatorname{sum}\left(\mathrm{g} 3323 \mathrm{a}(\mathrm{i}) \cdot{ }^{*} \mathrm{Wq}\right)+\operatorname{sum}(\mathrm{g} 33\right.\right.$ $23 \mathrm{~b}(\mathrm{i}) \cdot * \mathrm{Wq})))) /(8 * \mathrm{pi})$;

$\mathrm{S} 2313$

$\left(\left(\mathrm{L}(1,8) *\left(\operatorname{sum}\left(\mathrm{g} 2131 \mathrm{a}(\mathrm{i}) .{ }^{*} \mathrm{Wq}\right)+\operatorname{sum}\left(\mathrm{g} 2131 \mathrm{~b}(\mathrm{i}) .{ }^{*} \mathrm{Wq}\right)+\operatorname{sum}\left(\mathrm{g} 3121 \mathrm{a}(\mathrm{i}) .{ }^{*} \mathrm{Wq}\right)+\operatorname{sum}(\mathrm{g} 3\right.\right.\right.$ $121 \mathrm{~b}(\mathrm{i}) * \mathrm{Wq})))$

$\left(\mathrm{L}(6,8) *\left(\operatorname{sum}\left(\mathrm{g} 2132 \mathrm{a}(\mathrm{i}) .{ }^{*} \mathrm{Wq}\right)+\operatorname{sum}\left(\mathrm{g} 2132 \mathrm{~b}(\mathrm{i}) .{ }^{*} \mathrm{Wq}\right)+\operatorname{sum}\left(\mathrm{g} 3122 \mathrm{a}(\mathrm{i}) .{ }^{*} \mathrm{Wq}\right)+\operatorname{sum}(\mathrm{g} 31\right.\right.$ $22 \mathrm{~b}(\mathrm{i}) . * \mathrm{Wq})))$

$\left(\mathrm{L}(8,8) *\left(\operatorname{sum}\left(\mathrm{g} 2133 \mathrm{a}(\mathrm{i}) .{ }^{*} \mathrm{Wq}\right)+\operatorname{sum}\left(\mathrm{g} 2133 \mathrm{~b}(\mathrm{i}) .{ }^{*} \mathrm{Wq}\right)+\operatorname{sum}\left(\mathrm{g} 3123 \mathrm{a}(\mathrm{i}) .{ }^{*} \mathrm{Wq}\right)+\operatorname{sum}(\mathrm{g} 31\right.\right.$ $23 \mathrm{~b}(\mathrm{i}) . * \mathrm{Wq})))$

$\left(\mathrm{L}(9,8) *\left(\operatorname{sum}\left(\mathrm{g} 2231 \mathrm{a}(\mathrm{i}) .{ }^{*} \mathrm{Wq}\right)+\operatorname{sum}(\mathrm{g} 2231 \mathrm{~b}(\mathrm{i}) . * \mathrm{Wq})+\operatorname{sum}\left(\mathrm{g} 3221 \mathrm{a}(\mathrm{i}) .{ }^{*} \mathrm{Wq}\right)+\operatorname{sum}(\mathrm{g} 32\right.\right.$ $21 \mathrm{~b}(\mathrm{i}) . * \mathrm{Wq})))$

$\left(\mathrm{L}(2,8) *\left(\operatorname{sum}\left(\mathrm{g} 2232 \mathrm{a}(\mathrm{i}) .{ }^{*} \mathrm{Wq}\right)+\operatorname{sum}(\mathrm{g} 2232 \mathrm{~b}(\mathrm{i}) . * \mathrm{Wq})+\operatorname{sum}\left(\mathrm{g} 3222 \mathrm{a}(\mathrm{i}) .{ }^{*} \mathrm{Wq}\right)+\operatorname{sum}(\mathrm{g} 32\right.\right.$ $\left.\left.\left.22 \mathrm{~b}(\mathrm{i}) *{ }^{*} \mathrm{Wq}\right)\right)\right)$

$\left(\mathrm{L}(4,8) *\left(\operatorname{sum}\left(\mathrm{g} 2233 \mathrm{a}(\mathrm{i}) .{ }^{*} \mathrm{Wq}\right)+\operatorname{sum}\left(\mathrm{g} 2233 \mathrm{~b}(\mathrm{i}) \cdot{ }^{*} \mathrm{Wq}\right)+\operatorname{sum}\left(\mathrm{g} 3223 \mathrm{a}(\mathrm{i}) .{ }^{*} \mathrm{Wq}\right)+\operatorname{sum}(\mathrm{g} 32\right.\right.$ 23b(i).*Wq)))

$\left(\mathrm{L}(5,8) *\left(\operatorname{sum}\left(\mathrm{g} 2331 \mathrm{a}(\mathrm{i}) .{ }^{*} \mathrm{Wq}\right)+\operatorname{sum}\left(\mathrm{g} 2331 \mathrm{~b}(\mathrm{i}) \cdot{ }^{*} \mathrm{Wq}\right)+\operatorname{sum}(\mathrm{g} 3321 \mathrm{a}(\mathrm{i}) . * \mathrm{Wq})+\operatorname{sum}(\mathrm{g} 33\right.\right.$ $21 \mathrm{~b}(\mathrm{i}) . * \mathrm{Wq})))$

$\left(\mathrm{L}(7,8) *\left(\operatorname{sum}\left(\mathrm{g} 2332 \mathrm{a}(\mathrm{i}) .{ }^{*} \mathrm{Wq}\right)+\operatorname{sum}(\mathrm{g} 2332 \mathrm{~b}(\mathrm{i}) . * \mathrm{Wq})+\operatorname{sum}\left(\mathrm{g} 3322 \mathrm{a}(\mathrm{i}) .{ }^{*} \mathrm{Wq}\right)+\operatorname{sum}(\mathrm{g} 33\right.\right.$ $22 \mathrm{~b}(\mathrm{i}) . * \mathrm{Wq})))$

$\left(\mathrm{L}(3,8) *\left(\operatorname{sum}(\mathrm{g} 2333 \mathrm{a}(\mathrm{i}) . * \mathrm{Wq})+\operatorname{sum}\left(\mathrm{g} 2333 \mathrm{~b}(\mathrm{i}) \cdot{ }^{*} \mathrm{Wq}\right)+\operatorname{sum}\left(\mathrm{g} 3323 \mathrm{a}(\mathrm{i}) \cdot{ }^{*} \mathrm{Wq}\right)+\operatorname{sum}(\mathrm{g} 33\right.\right.$ $23 \mathrm{~b}(\mathrm{i}) . * \mathrm{Wq})))) /(8 * \mathrm{pi})$; 
S2321

$\left(\left(\mathrm{L}(1,9) *\left(\operatorname{sum}\left(\mathrm{g} 2131 \mathrm{a}(\mathrm{i}) .{ }^{*} \mathrm{Wq}\right)+\operatorname{sum}\left(\mathrm{g} 2131 \mathrm{~b}(\mathrm{i}) .{ }^{*} \mathrm{Wq}\right)+\operatorname{sum}\left(\mathrm{g} 3121 \mathrm{a}(\mathrm{i}) .{ }^{*} \mathrm{Wq}\right)+\operatorname{sum}(\mathrm{g} 3\right.\right.\right.$ $\left.\left.\left.121 \mathrm{~b}(\mathrm{i}) \cdot{ }^{*} \mathrm{Wq}\right)\right)\right)$

$\left(\mathrm{L}(6,9) *\left(\operatorname{sum}(\mathrm{g} 2132 \mathrm{a}(\mathrm{i}) . * \mathrm{Wq})+\operatorname{sum}\left(\mathrm{g} 2132 \mathrm{~b}(\mathrm{i}) \cdot{ }^{*} \mathrm{Wq}\right)+\operatorname{sum}\left(\mathrm{g} 3122 \mathrm{a}(\mathrm{i}) \cdot{ }^{*} \mathrm{Wq}\right)+\operatorname{sum}(\mathrm{g} 31\right.\right.$ $\left.\left.\left.22 \mathrm{~b}(\mathrm{i}) \cdot{ }^{*} \mathrm{Wq}\right)\right)\right)$

$\left(\mathrm{L}(8,9) *\left(\operatorname{sum}\left(\mathrm{g} 2133 \mathrm{a}(\mathrm{i}) .{ }^{*} \mathrm{Wq}\right)+\operatorname{sum}\left(\mathrm{g} 2133 \mathrm{~b}(\mathrm{i}) .{ }^{*} \mathrm{Wq}\right)+\operatorname{sum}(\mathrm{g} 3123 \mathrm{a}(\mathrm{i}) . * \mathrm{Wq})+\operatorname{sum}(\mathrm{g} 31\right.\right.$ $23 \mathrm{~b}(\mathrm{i}) \cdot * \mathrm{Wq}))$

$\left(\mathrm{L}(9,9) *\left(\operatorname{sum}\left(\mathrm{g} 2231 \mathrm{a}(\mathrm{i}) .{ }^{*} \mathrm{Wq}\right)+\operatorname{sum}\left(\mathrm{g} 2231 \mathrm{~b}(\mathrm{i}) \cdot{ }^{*} \mathrm{Wq}\right)+\operatorname{sum}\left(\mathrm{g} 3221 \mathrm{a}(\mathrm{i}) .{ }^{*} \mathrm{Wq}\right)+\operatorname{sum}(\mathrm{g} 32\right.\right.$ $21 \mathrm{~b}(\mathrm{i}) . * \mathrm{Wq}))$

$\left(\mathrm{L}(2,9) *\left(\operatorname{sum}\left(\mathrm{g} 2232 \mathrm{a}(\mathrm{i}) .{ }^{*} \mathrm{Wq}\right)+\operatorname{sum}\left(\mathrm{g} 2232 \mathrm{~b}(\mathrm{i}) \cdot{ }^{*} \mathrm{Wq}\right)+\operatorname{sum}(\mathrm{g} 3222 \mathrm{a}(\mathrm{i}) . * \mathrm{Wq})+\operatorname{sum}(\mathrm{g} 32\right.\right.$ $22 \mathrm{~b}(\mathrm{i}) . * \mathrm{Wq})))$

$\left(\mathrm{L}(4,9) *\left(\operatorname{sum}(\mathrm{g} 2233 \mathrm{a}(\mathrm{i}) . * \mathrm{Wq})+\operatorname{sum}\left(\mathrm{g} 2233 \mathrm{~b}(\mathrm{i}) .{ }^{*} \mathrm{Wq}\right)+\operatorname{sum}\left(\mathrm{g} 3223 \mathrm{a}(\mathrm{i}) .{ }^{*} \mathrm{Wq}\right)+\operatorname{sum}(\mathrm{g} 32\right.\right.$ $23 \mathrm{~b}(\mathrm{i}) \cdot * \mathrm{Wq}))$

$\left(\mathrm{L}(5,9) *\left(\operatorname{sum}\left(\mathrm{g} 2331 \mathrm{a}(\mathrm{i}) .{ }^{*} \mathrm{Wq}\right)+\operatorname{sum}\left(\mathrm{g} 2331 \mathrm{~b}(\mathrm{i}) .{ }^{*} \mathrm{Wq}\right)+\operatorname{sum}\left(\mathrm{g} 3321 \mathrm{a}(\mathrm{i}) .{ }^{*} \mathrm{Wq}\right)+\operatorname{sum}(\mathrm{g} 33\right.\right.$ $21 \mathrm{~b}(\mathrm{i}) . * \mathrm{Wq})))$

$\left(\mathrm{L}(7,9) *\left(\operatorname{sum}(\mathrm{g} 2332 \mathrm{a}(\mathrm{i}) . * \mathrm{Wq})+\operatorname{sum}\left(\mathrm{g} 2332 \mathrm{~b}(\mathrm{i}) \cdot{ }^{*} \mathrm{Wq}\right)+\operatorname{sum}(\mathrm{g} 3322 \mathrm{a}(\mathrm{i}) . * \mathrm{Wq})+\operatorname{sum}(\mathrm{g} 33\right.\right.$ $22 \mathrm{~b}(\mathrm{i}) \cdot * \mathrm{Wq})))$

$\left(\mathrm{L}(3,9) *\left(\operatorname{sum}\left(\mathrm{g} 2333 \mathrm{a}(\mathrm{i}) .{ }^{*} \mathrm{Wq}\right)+\operatorname{sum}\left(\mathrm{g} 2333 \mathrm{~b}(\mathrm{i}) \cdot{ }^{*} \mathrm{Wq}\right)+\operatorname{sum}\left(\mathrm{g} 3323 \mathrm{a}(\mathrm{i}) \cdot{ }^{*} \mathrm{Wq}\right)+\operatorname{sum}(\mathrm{g} 33\right.\right.$

$\left.\left.\left.\left.23 \mathrm{~b}(\mathrm{i}){ }^{*} \mathrm{Wq}\right)\right)\right)\right) /(8 * \mathrm{pi})$;

S3111

$\left(\left(\mathrm{L}(1,1) *\left(\operatorname{sum}\left(\mathrm{g} 3111 \mathrm{a}(\mathrm{i}) .{ }^{*} \mathrm{Wq}\right)+\operatorname{sum}\left(\mathrm{g} 3111 \mathrm{~b}(\mathrm{i}) .{ }^{*} \mathrm{Wq}\right)+\operatorname{sum}\left(\mathrm{g} 1131 \mathrm{a}(\mathrm{i}) .{ }^{*} \mathrm{Wq}\right)+\operatorname{sum}(\mathrm{g} 1\right.\right.\right.$ $\left.\left.\left.131 \mathrm{~b}(\mathrm{i}) *{ }^{*} \mathrm{Wq}\right)\right)\right)$

$\left(\mathrm{L}(6,1) *\left(\operatorname{sum}\left(\mathrm{g} 3112 \mathrm{a}(\mathrm{i}) .{ }^{*} \mathrm{Wq}\right)+\operatorname{sum}\left(\mathrm{g} 3112 \mathrm{~b}(\mathrm{i}) \cdot{ }^{*} \mathrm{Wq}\right)+\operatorname{sum}\left(\mathrm{g} 1132 \mathrm{a}(\mathrm{i}) .{ }^{*} \mathrm{Wq}\right)+\operatorname{sum}(\mathrm{g} 11\right.\right.$ $32 \mathrm{~b}(\mathrm{i}) . * \mathrm{Wq})))$

$\left(\mathrm{L}(8,1) *\left(\operatorname{sum}\left(\mathrm{g} 3113 \mathrm{a}(\mathrm{i}) .{ }^{*} \mathrm{Wq}\right)+\operatorname{sum}\left(\mathrm{g} 3113 \mathrm{~b}(\mathrm{i}) .{ }^{*} \mathrm{Wq}\right)+\operatorname{sum}\left(\mathrm{g} 1133 \mathrm{a}(\mathrm{i}) .{ }^{*} \mathrm{Wq}\right)+\operatorname{sum}(\mathrm{g} 11\right.\right.$ $33 \mathrm{~b}(\mathrm{i}) . * \mathrm{Wq})))$

$\left(\mathrm{L}(9,1) *\left(\operatorname{sum}\left(\mathrm{g} 3211 \mathrm{a}(\mathrm{i}) .{ }^{*} \mathrm{Wq}\right)+\operatorname{sum}\left(\mathrm{g} 3211 \mathrm{~b}(\mathrm{i}) .{ }^{*} \mathrm{Wq}\right)+\operatorname{sum}\left(\mathrm{g} 1231 \mathrm{a}(\mathrm{i}) .{ }^{*} \mathrm{Wq}\right)+\operatorname{sum}(\mathrm{g} 12\right.\right.$

$31 \mathrm{~b}(\mathrm{i}) . * \mathrm{Wq})))$

$\left(\mathrm{L}(2,1) *\left(\operatorname{sum}\left(\mathrm{g} 3212 \mathrm{a}(\mathrm{i}) .{ }^{*} \mathrm{Wq}\right)+\operatorname{sum}\left(\mathrm{g} 3212 \mathrm{~b}(\mathrm{i}) .{ }^{*} \mathrm{Wq}\right)+\operatorname{sum}\left(\mathrm{g} 1232 \mathrm{a}(\mathrm{i}) .{ }^{*} \mathrm{Wq}\right)+\operatorname{sum}(\mathrm{g} 12\right.\right.$

$32 \mathrm{~b}(\mathrm{i}) . * \mathrm{Wq})))$

$\left(\mathrm{L}(4,1) *\left(\operatorname{sum}\left(\mathrm{g} 3213 \mathrm{a}(\mathrm{i}) .{ }^{*} \mathrm{Wq}\right)+\operatorname{sum}\left(\mathrm{g} 3213 \mathrm{~b}(\mathrm{i}) .{ }^{*} \mathrm{Wq}\right)+\operatorname{sum}\left(\mathrm{g} 1233 \mathrm{a}(\mathrm{i}) .{ }^{*} \mathrm{Wq}\right)+\operatorname{sum}(\mathrm{g} 12\right.\right.$ $\left.\left.33 \mathrm{~b}(\mathrm{i}) \cdot{ }^{*} \mathrm{Wq}\right)\right)$

$\left(\mathrm{L}(5,1) *\left(\operatorname{sum}\left(\mathrm{g} 3311 \mathrm{a}(\mathrm{i}) .{ }^{*} \mathrm{Wq}\right)+\operatorname{sum}\left(\mathrm{g} 3311 \mathrm{~b}(\mathrm{i}) .{ }^{*} \mathrm{Wq}\right)+\operatorname{sum}(\mathrm{g} 1331 \mathrm{a}(\mathrm{i}) . * \mathrm{Wq})+\operatorname{sum}(\mathrm{g} 13\right.\right.$ $31 \mathrm{~b}(\mathrm{i}) . * \mathrm{Wq})))$

$(\mathrm{L}(7,1) *(\operatorname{sum}(\mathrm{g} 3312 \mathrm{a}(\mathrm{i}) . * \mathrm{Wq})+\operatorname{sum}(\mathrm{g} 3312 \mathrm{~b}(\mathrm{i}) . * \mathrm{Wq})+\operatorname{sum}(\mathrm{g} 1332 \mathrm{a}(\mathrm{i}) . * \mathrm{Wq})+\operatorname{sum}(\mathrm{g} 13$ $32 \mathrm{~b}(\mathrm{i}) . * \mathrm{Wq}))$

$\left(\mathrm{L}(3,1) *\left(\operatorname{sum}\left(\mathrm{g} 3313 \mathrm{a}(\mathrm{i}) .{ }^{*} \mathrm{Wq}\right)+\operatorname{sum}\left(\mathrm{g} 3313 \mathrm{~b}(\mathrm{i}) .{ }^{*} \mathrm{Wq}\right)+\operatorname{sum}\left(\mathrm{g} 1333 \mathrm{a}(\mathrm{i}) .{ }^{*} \mathrm{Wq}\right)+\operatorname{sum}(\mathrm{g} 13\right.\right.$

$33 \mathrm{~b}(\mathrm{i}) *$ *Wq $))) /(8 * \mathrm{pi})$

S3122

$\left(\left(\mathrm{L}(1,2) *\left(\operatorname{sum}\left(\mathrm{g} 3111 \mathrm{a}(\mathrm{i}) .{ }^{*} \mathrm{Wq}\right)+\operatorname{sum}\left(\mathrm{g} 3111 \mathrm{~b}(\mathrm{i}) .{ }^{*} \mathrm{Wq}\right)+\operatorname{sum}\left(\mathrm{g} 1131 \mathrm{a}(\mathrm{i}) .{ }^{*} \mathrm{Wq}\right)+\operatorname{sum}(\mathrm{g} 1\right.\right.\right.$ $131 \mathrm{~b}(\mathrm{i}) * \mathrm{Wq})))$

$\left(\mathrm{L}(6,2) *\left(\operatorname{sum}\left(\mathrm{g} 3112 \mathrm{a}(\mathrm{i}) .{ }^{*} \mathrm{Wq}\right)+\operatorname{sum}\left(\mathrm{g} 3112 \mathrm{~b}(\mathrm{i}) \cdot{ }^{*} \mathrm{Wq}\right)+\operatorname{sum}\left(\mathrm{g} 1132 \mathrm{a}(\mathrm{i}) \cdot{ }^{*} \mathrm{Wq}\right)+\operatorname{sum}(\mathrm{g} 11\right.\right.$ 
$32 \mathrm{~b}(\mathrm{i}) \cdot * \mathrm{Wq})))$

$\left(\mathrm{L}(8,2) *\left(\operatorname{sum}\left(\mathrm{g} 3113 \mathrm{a}(\mathrm{i}) .{ }^{*} \mathrm{Wq}\right)+\operatorname{sum}\left(\mathrm{g} 3113 \mathrm{~b}(\mathrm{i}) .{ }^{*} \mathrm{Wq}\right)+\operatorname{sum}\left(\mathrm{g} 1133 \mathrm{a}(\mathrm{i}) .{ }^{*} \mathrm{Wq}\right)+\operatorname{sum}(\mathrm{g} 11\right.\right.$

$33 \mathrm{~b}(\mathrm{i}) . * \mathrm{Wq}))$ )

$\left(\mathrm{L}(9,2) *\left(\operatorname{sum}\left(\mathrm{g} 3211 \mathrm{a}(\mathrm{i}) .{ }^{*} \mathrm{Wq}\right)+\operatorname{sum}\left(\mathrm{g} 3211 \mathrm{~b}(\mathrm{i}) \cdot{ }^{*} \mathrm{Wq}\right)+\operatorname{sum}\left(\mathrm{g} 1231 \mathrm{a}(\mathrm{i}) \cdot{ }^{*} \mathrm{Wq}\right)+\operatorname{sum}(\mathrm{g} 12\right.\right.$

$\left.\left.31 \mathrm{~b}(\mathrm{i}) \cdot{ }^{*} \mathrm{Wq}\right)\right)$ )

$\left(\mathrm{L}(2,2) *\left(\operatorname{sum}\left(\mathrm{g} 3212 \mathrm{a}(\mathrm{i}) .{ }^{*} \mathrm{Wq}\right)+\operatorname{sum}\left(\mathrm{g} 3212 \mathrm{~b}(\mathrm{i}) .{ }^{*} \mathrm{Wq}\right)+\operatorname{sum}\left(\mathrm{g} 1232 \mathrm{a}(\mathrm{i}) .{ }^{*} \mathrm{Wq}\right)+\operatorname{sum}(\mathrm{g} 12\right.\right.$

$32 \mathrm{~b}(\mathrm{i}) . * \mathrm{Wq})))$

$\left(\mathrm{L}(4,2) *\left(\operatorname{sum}\left(\mathrm{g} 3213 \mathrm{a}(\mathrm{i}) .{ }^{*} \mathrm{Wq}\right)+\operatorname{sum}\left(\mathrm{g} 3213 \mathrm{~b}(\mathrm{i}) .{ }^{*} \mathrm{Wq}\right)+\operatorname{sum}\left(\mathrm{g} 1233 \mathrm{a}(\mathrm{i}) .{ }^{*} \mathrm{Wq}\right)+\operatorname{sum}(\mathrm{g} 12\right.\right.$ $\left.\left.33 \mathrm{~b}(\mathrm{i}) \cdot{ }^{*} \mathrm{Wq}\right)\right)$

$\left(\mathrm{L}(5,2) *\left(\operatorname{sum}\left(\mathrm{g} 3311 \mathrm{a}(\mathrm{i}) .{ }^{*} \mathrm{Wq}\right)+\operatorname{sum}\left(\mathrm{g} 3311 \mathrm{~b}(\mathrm{i}) .{ }^{*} \mathrm{Wq}\right)+\operatorname{sum}\left(\mathrm{g} 1331 \mathrm{a}(\mathrm{i}) .{ }^{*} \mathrm{Wq}\right)+\operatorname{sum}(\mathrm{g} 13\right.\right.$ $31 \mathrm{~b}(\mathrm{i}) . * \mathrm{Wq})))$

$\left(\mathrm{L}(7,2) *\left(\operatorname{sum}(\mathrm{g} 3312 \mathrm{a}(\mathrm{i}) . * \mathrm{Wq})+\operatorname{sum}\left(\mathrm{g} 3312 \mathrm{~b}(\mathrm{i}) .{ }^{*} \mathrm{Wq}\right)+\operatorname{sum}(\mathrm{g} 1332 \mathrm{a}(\mathrm{i}) . * \mathrm{Wq})+\operatorname{sum}(\mathrm{g} 13\right.\right.$

$32 \mathrm{~b}(\mathrm{i}) \cdot * \mathrm{Wq}))$

$\left(\mathrm{L}(3,2) *\left(\operatorname{sum}\left(\mathrm{g} 3313 \mathrm{a}(\mathrm{i}) .{ }^{*} \mathrm{Wq}\right)+\operatorname{sum}\left(\mathrm{g} 3313 \mathrm{~b}(\mathrm{i}) \cdot{ }^{*} \mathrm{Wq}\right)+\operatorname{sum}\left(\mathrm{g} 1333 \mathrm{a}(\mathrm{i}) .{ }^{*} \mathrm{Wq}\right)+\operatorname{sum}(\mathrm{g} 13\right.\right.$

$\left.\left.\left.\left.33 \mathrm{~b}(\mathrm{i}) \cdot{ }^{*} \mathrm{Wq}\right)\right)\right)\right) /(8 * \mathrm{pi})$;

S3133

$\left(\left(\mathrm{L}(1,3) *\left(\operatorname{sum}\left(\mathrm{g} 3111 \mathrm{a}(\mathrm{i}) .{ }^{*} \mathrm{Wq}\right)+\operatorname{sum}\left(\mathrm{g} 3111 \mathrm{~b}(\mathrm{i}) .{ }^{*} \mathrm{Wq}\right)+\operatorname{sum}\left(\mathrm{g} 1131 \mathrm{a}(\mathrm{i}) .{ }^{*} \mathrm{Wq}\right)+\operatorname{sum}(\mathrm{g} 1\right.\right.\right.$ $131 \mathrm{~b}(\mathrm{i}) * \mathrm{Wq})))$

$\left(\mathrm{L}(6,3) *\left(\operatorname{sum}\left(\mathrm{g} 3112 \mathrm{a}(\mathrm{i}) .{ }^{*} \mathrm{Wq}\right)+\operatorname{sum}\left(\mathrm{g} 3112 \mathrm{~b}(\mathrm{i}) .{ }^{*} \mathrm{Wq}\right)+\operatorname{sum}\left(\mathrm{g} 1132 \mathrm{a}(\mathrm{i}) .{ }^{*} \mathrm{Wq}\right)+\operatorname{sum}(\mathrm{g} 11\right.\right.$ $32 \mathrm{~b}(\mathrm{i}) . * \mathrm{Wq})))$

$\left(\mathrm{L}(8,3) *\left(\operatorname{sum}\left(\mathrm{g} 3113 \mathrm{a}(\mathrm{i}) .{ }^{*} \mathrm{Wq}\right)+\operatorname{sum}\left(\mathrm{g} 3113 \mathrm{~b}(\mathrm{i}) \cdot{ }^{*} \mathrm{Wq}\right)+\operatorname{sum}\left(\mathrm{g} 1133 \mathrm{a}(\mathrm{i}) .{ }^{*} \mathrm{Wq}\right)+\operatorname{sum}(\mathrm{g} 11\right.\right.$

$33 \mathrm{~b}(\mathrm{i}) . * \mathrm{Wq})))$

$\left(\mathrm{L}(9,3) *\left(\operatorname{sum}\left(\mathrm{g} 3211 \mathrm{a}(\mathrm{i}) .{ }^{*} \mathrm{Wq}\right)+\operatorname{sum}\left(\mathrm{g} 3211 \mathrm{~b}(\mathrm{i}) .{ }^{*} \mathrm{Wq}\right)+\operatorname{sum}\left(\mathrm{g} 1231 \mathrm{a}(\mathrm{i}) .{ }^{*} \mathrm{Wq}\right)+\operatorname{sum}(\mathrm{g} 12\right.\right.$

$31 \mathrm{~b}(\mathrm{i}) . * \mathrm{Wq})))$

$\left(\mathrm{L}(2,3) *\left(\operatorname{sum}\left(\mathrm{g} 3212 \mathrm{a}(\mathrm{i}) .{ }^{*} \mathrm{Wq}\right)+\operatorname{sum}\left(\mathrm{g} 3212 \mathrm{~b}(\mathrm{i}) \cdot{ }^{*} \mathrm{Wq}\right)+\operatorname{sum}\left(\mathrm{g} 1232 \mathrm{a}(\mathrm{i}) \cdot{ }^{*} \mathrm{Wq}\right)+\operatorname{sum}(\mathrm{g} 12\right.\right.$

$32 \mathrm{~b}(\mathrm{i}) . * \mathrm{Wq})))$

$\left(\mathrm{L}(4,3) *\left(\operatorname{sum}\left(\mathrm{g} 3213 \mathrm{a}(\mathrm{i}) .{ }^{*} \mathrm{Wq}\right)+\operatorname{sum}\left(\mathrm{g} 3213 \mathrm{~b}(\mathrm{i}) .{ }^{*} \mathrm{Wq}\right)+\operatorname{sum}\left(\mathrm{g} 1233 \mathrm{a}(\mathrm{i}) .{ }^{*} \mathrm{Wq}\right)+\operatorname{sum}(\mathrm{g} 12\right.\right.$

$\left.\left.33 \mathrm{~b}(\mathrm{i}) \cdot{ }^{*} \mathrm{Wq}\right)\right)$ )

$\left(\mathrm{L}(5,3) *\left(\operatorname{sum}\left(\mathrm{g} 3311 \mathrm{a}(\mathrm{i}) .{ }^{*} \mathrm{Wq}\right)+\operatorname{sum}\left(\mathrm{g} 3311 \mathrm{~b}(\mathrm{i}) \cdot{ }^{*} \mathrm{Wq}\right)+\operatorname{sum}\left(\mathrm{g} 1331 \mathrm{a}(\mathrm{i}) .{ }^{*} \mathrm{Wq}\right)+\operatorname{sum}(\mathrm{g} 13\right.\right.$

$31 \mathrm{~b}(\mathrm{i}) . * \mathrm{Wq})))$

$\left(\mathrm{L}(7,3) *\left(\operatorname{sum}\left(\mathrm{g} 3312 \mathrm{a}(\mathrm{i}) .{ }^{*} \mathrm{Wq}\right)+\operatorname{sum}\left(\mathrm{g} 3312 \mathrm{~b}(\mathrm{i}) .{ }^{*} \mathrm{Wq}\right)+\operatorname{sum}(\mathrm{g} 1332 \mathrm{a}(\mathrm{i}) . * \mathrm{Wq})+\operatorname{sum}(\mathrm{g} 13\right.\right.$

32b(i).*Wq))) +

$\left(\mathrm{L}(3,3) *\left(\operatorname{sum}\left(\mathrm{g} 3313 \mathrm{a}(\mathrm{i}) .{ }^{*} \mathrm{Wq}\right)+\operatorname{sum}\left(\mathrm{g} 3313 \mathrm{~b}(\mathrm{i}) .{ }^{*} \mathrm{Wq}\right)+\operatorname{sum}\left(\mathrm{g} 1333 \mathrm{a}(\mathrm{i}) .{ }^{*} \mathrm{Wq}\right)+\operatorname{sum}(\mathrm{g} 13\right.\right.$

$\left.\left.\left.\left.33 \mathrm{~b}(\mathrm{i}) \cdot{ }^{*} \mathrm{Wq}\right)\right)\right)\right) /(8 * \mathrm{pi})$;

S3123

$((\mathrm{L}(1,4) *(\operatorname{sum}(\mathrm{g} 3111 \mathrm{a}(\mathrm{i}) . * \mathrm{Wq})+\operatorname{sum}(\mathrm{g} 3111 \mathrm{~b}(\mathrm{i}) . * \mathrm{Wq})+\operatorname{sum}(\mathrm{g} 1131 \mathrm{a}(\mathrm{i}) . * \mathrm{Wq})+\operatorname{sum}(\mathrm{g} 1$ $131 \mathrm{~b}(\mathrm{i}) * \mathrm{Wq})))$

$\left(\mathrm{L}(6,4) *\left(\operatorname{sum}\left(\mathrm{g} 3112 \mathrm{a}(\mathrm{i}) .{ }^{*} \mathrm{Wq}\right)+\operatorname{sum}\left(\mathrm{g} 3112 \mathrm{~b}(\mathrm{i}) .{ }^{*} \mathrm{Wq}\right)+\operatorname{sum}\left(\mathrm{g} 1132 \mathrm{a}(\mathrm{i}) .{ }^{*} \mathrm{Wq}\right)+\operatorname{sum}(\mathrm{g} 11\right.\right.$ $32 \mathrm{~b}(\mathrm{i}) . * \mathrm{Wq})))$

$\left(\mathrm{L}(8,4) *\left(\operatorname{sum}\left(\mathrm{g} 3113 \mathrm{a}(\mathrm{i}) .{ }^{*} \mathrm{Wq}\right)+\operatorname{sum}\left(\mathrm{g} 3113 \mathrm{~b}(\mathrm{i}) .{ }^{*} \mathrm{Wq}\right)+\operatorname{sum}\left(\mathrm{g} 1133 \mathrm{a}(\mathrm{i}) .{ }^{*} \mathrm{Wq}\right)+\operatorname{sum}(\mathrm{g} 11\right.\right.$

$33 \mathrm{~b}(\mathrm{i}) . * \mathrm{Wq}))$

$\left(\mathrm{L}(9,4) *\left(\operatorname{sum}\left(\mathrm{g} 3211 \mathrm{a}(\mathrm{i}) .{ }^{*} \mathrm{Wq}\right)+\operatorname{sum}\left(\mathrm{g} 3211 \mathrm{~b}(\mathrm{i}) .{ }^{*} \mathrm{Wq}\right)+\operatorname{sum}\left(\mathrm{g} 1231 \mathrm{a}(\mathrm{i}) .{ }^{*} \mathrm{Wq}\right)+\operatorname{sum}(\mathrm{g} 12\right.\right.$

$\left.\left.\left.31 \mathrm{~b}(\mathrm{i}) \cdot{ }^{*} \mathrm{Wq}\right)\right)\right)$ 
$\left(\mathrm{L}(2,4) *\left(\operatorname{sum}\left(\mathrm{g} 3212 \mathrm{a}(\mathrm{i}) .{ }^{*} \mathrm{Wq}\right)+\operatorname{sum}\left(\mathrm{g} 3212 \mathrm{~b}(\mathrm{i}) \cdot{ }^{*} \mathrm{Wq}\right)+\operatorname{sum}\left(\mathrm{g} 1232 \mathrm{a}(\mathrm{i}) .{ }^{*} \mathrm{Wq}\right)+\operatorname{sum}(\mathrm{g} 12\right.\right.$ $32 \mathrm{~b}(\mathrm{i}) . * \mathrm{Wq})))$

$\left(\mathrm{L}(4,4) *\left(\operatorname{sum}(\mathrm{g} 3213 \mathrm{a}(\mathrm{i}) . * \mathrm{Wq})+\operatorname{sum}\left(\mathrm{g} 3213 \mathrm{~b}(\mathrm{i}) \cdot{ }^{*} \mathrm{Wq}\right)+\operatorname{sum}\left(\mathrm{g} 1233 \mathrm{a}(\mathrm{i}) .{ }^{*} \mathrm{Wq}\right)+\operatorname{sum}(\mathrm{g} 12\right.\right.$ $\left.\left.\left.33 \mathrm{~b}(\mathrm{i}) *{ }^{*} \mathrm{Wq}\right)\right)\right)$

$\left(\mathrm{L}(5,4) *\left(\operatorname{sum}\left(\mathrm{g} 3311 \mathrm{a}(\mathrm{i}) .{ }^{*} \mathrm{Wq}\right)+\operatorname{sum}\left(\mathrm{g} 3311 \mathrm{~b}(\mathrm{i}) .{ }^{*} \mathrm{Wq}\right)+\operatorname{sum}\left(\mathrm{g} 1331 \mathrm{a}(\mathrm{i}) .{ }^{*} \mathrm{Wq}\right)+\operatorname{sum}(\mathrm{g} 13\right.\right.$ $31 \mathrm{~b}(\mathrm{i}) . * \mathrm{Wq})))$ $\left(\mathrm{L}(7,4) *\left(\operatorname{sum}\left(\mathrm{g} 3312 \mathrm{a}(\mathrm{i}) .{ }^{*} \mathrm{Wq}\right)+\operatorname{sum}\left(\mathrm{g} 3312 \mathrm{~b}(\mathrm{i}) \cdot{ }^{*} \mathrm{Wq}\right)+\operatorname{sum}\left(\mathrm{g} 1332 \mathrm{a}(\mathrm{i}) .{ }^{*} \mathrm{Wq}\right)+\operatorname{sum}(\mathrm{g} 13\right.\right.$ $32 \mathrm{~b}(\mathrm{i}) . * \mathrm{Wq})))$ $\left(\mathrm{L}(3,4) *\left(\operatorname{sum}\left(\mathrm{g} 3313 \mathrm{a}(\mathrm{i}) .{ }^{*} \mathrm{Wq}\right)+\operatorname{sum}\left(\mathrm{g} 3313 \mathrm{~b}(\mathrm{i}) .{ }^{*} \mathrm{Wq}\right)+\operatorname{sum}\left(\mathrm{g} 1333 \mathrm{a}(\mathrm{i}) .{ }^{*} \mathrm{Wq}\right)+\operatorname{sum}(\mathrm{g} 13\right.\right.$ $33 \mathrm{~b}(\mathrm{i}) . * \mathrm{Wq})))) /(8 * \mathrm{pi})$;

S3131

$\left(\left(\mathrm{L}(1,5) *\left(\operatorname{sum}\left(\mathrm{g} 3111 \mathrm{a}(\mathrm{i}) .{ }^{*} \mathrm{Wq}\right)+\operatorname{sum}\left(\mathrm{g} 3111 \mathrm{~b}(\mathrm{i}) .{ }^{*} \mathrm{Wq}\right)+\operatorname{sum}\left(\mathrm{g} 1131 \mathrm{a}(\mathrm{i}) .{ }^{*} \mathrm{Wq}\right)+\operatorname{sum}(\mathrm{g} 1\right.\right.\right.$ $\left.\left.\left.131 \mathrm{~b}(\mathrm{i}) \cdot{ }^{*} \mathrm{Wq}\right)\right)\right)$

$\left(\mathrm{L}(6,5) *\left(\operatorname{sum}\left(\mathrm{g} 3112 \mathrm{a}(\mathrm{i}) .{ }^{*} \mathrm{Wq}\right)+\operatorname{sum}\left(\mathrm{g} 3112 \mathrm{~b}(\mathrm{i}) \cdot{ }^{*} \mathrm{Wq}\right)+\operatorname{sum}\left(\mathrm{g} 1132 \mathrm{a}(\mathrm{i}) \cdot{ }^{*} \mathrm{Wq}\right)+\operatorname{sum}(\mathrm{g} 11\right.\right.$ $32 \mathrm{~b}(\mathrm{i}) . * \mathrm{Wq}))$ )

$\left(\mathrm{L}(8,5) *\left(\operatorname{sum}\left(\mathrm{g} 3113 \mathrm{a}(\mathrm{i}) .{ }^{*} \mathrm{Wq}\right)+\operatorname{sum}\left(\mathrm{g} 3113 \mathrm{~b}(\mathrm{i}) .{ }^{*} \mathrm{Wq}\right)+\operatorname{sum}\left(\mathrm{g} 1133 \mathrm{a}(\mathrm{i}) .{ }^{*} \mathrm{Wq}\right)+\operatorname{sum}(\mathrm{g} 11\right.\right.$ $33 \mathrm{~b}(\mathrm{i}) . * \mathrm{Wq}))$ )

$\left(\mathrm{L}(9,5) *\left(\operatorname{sum}\left(\mathrm{g} 3211 \mathrm{a}(\mathrm{i}) .{ }^{*} \mathrm{Wq}\right)+\operatorname{sum}\left(\mathrm{g} 3211 \mathrm{~b}(\mathrm{i}) \cdot{ }^{*} \mathrm{Wq}\right)+\operatorname{sum}\left(\mathrm{g} 1231 \mathrm{a}(\mathrm{i}) .{ }^{*} \mathrm{Wq}\right)+\operatorname{sum}(\mathrm{g} 12\right.\right.$ $31 \mathrm{~b}(\mathrm{i}) . * \mathrm{Wq}))$ )

$\left(\mathrm{L}(2,5) *\left(\operatorname{sum}\left(\mathrm{g} 3212 \mathrm{a}(\mathrm{i}) .{ }^{*} \mathrm{Wq}\right)+\operatorname{sum}\left(\mathrm{g} 3212 \mathrm{~b}(\mathrm{i}) \cdot{ }^{*} \mathrm{Wq}\right)+\operatorname{sum}\left(\mathrm{g} 1232 \mathrm{a}(\mathrm{i}) .{ }^{*} \mathrm{Wq}\right)+\operatorname{sum}(\mathrm{g} 12\right.\right.$ $32 \mathrm{~b}(\mathrm{i}) . * \mathrm{Wq})))$

$\left(\mathrm{L}(4,5) *\left(\operatorname{sum}\left(\mathrm{g} 3213 \mathrm{a}(\mathrm{i}) .{ }^{*} \mathrm{Wq}\right)+\operatorname{sum}\left(\mathrm{g} 3213 \mathrm{~b}(\mathrm{i}) \cdot{ }^{*} \mathrm{Wq}\right)+\operatorname{sum}\left(\mathrm{g} 1233 \mathrm{a}(\mathrm{i}) \cdot{ }^{*} \mathrm{Wq}\right)+\operatorname{sum}(\mathrm{g} 12\right.\right.$ $\left.\left.\left.33 \mathrm{~b}(\mathrm{i}) *{ }^{*} \mathrm{Wq}\right)\right)\right)$

$\left(\mathrm{L}(5,5) *\left(\operatorname{sum}\left(\mathrm{g} 3311 \mathrm{a}(\mathrm{i}) .{ }^{*} \mathrm{Wq}\right)+\operatorname{sum}\left(\mathrm{g} 3311 \mathrm{~b}(\mathrm{i}) .{ }^{*} \mathrm{Wq}\right)+\operatorname{sum}\left(\mathrm{g} 1331 \mathrm{a}(\mathrm{i}) .{ }^{*} \mathrm{Wq}\right)+\operatorname{sum}(\mathrm{g} 13\right.\right.$ $31 \mathrm{~b}(\mathrm{i}) . * \mathrm{Wq})))$ $\left(\mathrm{L}(7,5) *\left(\operatorname{sum}\left(\mathrm{g} 3312 \mathrm{a}(\mathrm{i}) .{ }^{*} \mathrm{Wq}\right)+\operatorname{sum}\left(\mathrm{g} 3312 \mathrm{~b}(\mathrm{i}) \cdot{ }^{*} \mathrm{Wq}\right)+\operatorname{sum}\left(\mathrm{g} 1332 \mathrm{a}(\mathrm{i}) \cdot{ }^{*} \mathrm{Wq}\right)+\operatorname{sum}(\mathrm{g} 13\right.\right.$ $32 \mathrm{~b}(\mathrm{i}) . * \mathrm{Wq})))$ $\left(\mathrm{L}(3,5) *\left(\operatorname{sum}(\mathrm{g} 3313 \mathrm{a}(\mathrm{i}) . * \mathrm{Wq})+\operatorname{sum}(\mathrm{g} 3313 \mathrm{~b}(\mathrm{i}) \cdot * \mathrm{Wq})+\operatorname{sum}\left(\mathrm{g} 1333 \mathrm{a}(\mathrm{i}) \cdot{ }^{*} \mathrm{Wq}\right)+\operatorname{sum}(\mathrm{g} 13\right.\right.$ $33 \mathrm{~b}(\mathrm{i}) *$ Wq $)))) /(8 * \mathrm{pi})$;

S3112

$\left(\left(\mathrm{L}(1,6) *\left(\operatorname{sum}\left(\mathrm{g} 3111 \mathrm{a}(\mathrm{i}) .{ }^{*} \mathrm{Wq}\right)+\operatorname{sum}\left(\mathrm{g} 3111 \mathrm{~b}(\mathrm{i}){ }^{*} \mathrm{Wq}\right)+\operatorname{sum}\left(\mathrm{g} 1131 \mathrm{a}(\mathrm{i}) .{ }^{*} \mathrm{Wq}\right)+\operatorname{sum}(\mathrm{g} 1\right.\right.\right.$ $131 \mathrm{~b}(\mathrm{i}) \cdot * \mathrm{Wq})))$

$\left(\mathrm{L}(6,6) *\left(\operatorname{sum}\left(\mathrm{g} 3112 \mathrm{a}(\mathrm{i}) .{ }^{*} \mathrm{Wq}\right)+\operatorname{sum}\left(\mathrm{g} 3112 \mathrm{~b}(\mathrm{i}) \cdot{ }^{*} \mathrm{Wq}\right)+\operatorname{sum}\left(\mathrm{g} 1132 \mathrm{a}(\mathrm{i}) .{ }^{*} \mathrm{Wq}\right)+\operatorname{sum}(\mathrm{g} 11\right.\right.$ $32 \mathrm{~b}(\mathrm{i}) . * \mathrm{Wq}))$ )

$\left(\mathrm{L}(8,6) *\left(\operatorname{sum}\left(\mathrm{g} 3113 \mathrm{a}(\mathrm{i}) .{ }^{*} \mathrm{Wq}\right)+\operatorname{sum}\left(\mathrm{g} 3113 \mathrm{~b}(\mathrm{i}) .{ }^{*} \mathrm{Wq}\right)+\operatorname{sum}\left(\mathrm{g} 1133 \mathrm{a}(\mathrm{i}) .{ }^{*} \mathrm{Wq}\right)+\operatorname{sum}(\mathrm{g} 11\right.\right.$ $\left.\left.33 \mathrm{~b}(\mathrm{i}){ }^{*} \mathrm{Wq}\right)\right)$ )

$\left(\mathrm{L}(9,6) *\left(\operatorname{sum}\left(\mathrm{g} 3211 \mathrm{a}(\mathrm{i}) .{ }^{*} \mathrm{Wq}\right)+\operatorname{sum}\left(\mathrm{g} 3211 \mathrm{~b}(\mathrm{i}) .{ }^{*} \mathrm{Wq}\right)+\operatorname{sum}\left(\mathrm{g} 1231 \mathrm{a}(\mathrm{i}) .{ }^{*} \mathrm{Wq}\right)+\operatorname{sum}(\mathrm{g} 12\right.\right.$ $31 \mathrm{~b}(\mathrm{i}) . * \mathrm{Wq}))$ )

$\left(\mathrm{L}(2,6) *\left(\operatorname{sum}\left(\mathrm{g} 3212 \mathrm{a}(\mathrm{i}) .{ }^{*} \mathrm{Wq}\right)+\operatorname{sum}\left(\mathrm{g} 3212 \mathrm{~b}(\mathrm{i}) .{ }^{*} \mathrm{Wq}\right)+\operatorname{sum}\left(\mathrm{g} 1232 \mathrm{a}(\mathrm{i}) .{ }^{*} \mathrm{Wq}\right)+\operatorname{sum}(\mathrm{g} 12\right.\right.$ $32 \mathrm{~b}(\mathrm{i}) . * \mathrm{Wq})))$

$\left(\mathrm{L}(4,6) *\left(\operatorname{sum}\left(\mathrm{g} 3213 \mathrm{a}(\mathrm{i}) .{ }^{*} \mathrm{Wq}\right)+\operatorname{sum}\left(\mathrm{g} 3213 \mathrm{~b}(\mathrm{i}) .{ }^{*} \mathrm{Wq}\right)+\operatorname{sum}\left(\mathrm{g} 1233 \mathrm{a}(\mathrm{i}) .{ }^{*} \mathrm{Wq}\right)+\operatorname{sum}(\mathrm{g} 12\right.\right.$ $33 \mathrm{~b}(\mathrm{i}) . * \mathrm{Wq}))$ )

$\left(\mathrm{L}(5,6) *\left(\operatorname{sum}\left(\mathrm{g} 3311 \mathrm{a}(\mathrm{i}) \cdot{ }^{*} \mathrm{Wq}\right)+\operatorname{sum}\left(\mathrm{g} 3311 \mathrm{~b}(\mathrm{i}) \cdot{ }^{*} \mathrm{Wq}\right)+\operatorname{sum}\left(\mathrm{g} 1331 \mathrm{a}(\mathrm{i}) \cdot{ }^{*} \mathrm{Wq}\right)+\operatorname{sum}(\mathrm{g} 13\right.\right.$ 
$31 \mathrm{~b}(\mathrm{i}) . * \mathrm{Wq})))$

$\left(\mathrm{L}(7,6) *\left(\operatorname{sum}\left(\mathrm{g} 3312 \mathrm{a}(\mathrm{i}) .{ }^{*} \mathrm{Wq}\right)+\operatorname{sum}\left(\mathrm{g} 3312 \mathrm{~b}(\mathrm{i}) .{ }^{*} \mathrm{Wq}\right)+\operatorname{sum}\left(\mathrm{g} 1332 \mathrm{a}(\mathrm{i}) .{ }^{*} \mathrm{Wq}\right)+\operatorname{sum}(\mathrm{g} 13\right.\right.$

$32 \mathrm{~b}(\mathrm{i}) . * \mathrm{Wq}))$ )

$\left(\mathrm{L}(3,6) *\left(\operatorname{sum}\left(\mathrm{g} 3313 \mathrm{a}(\mathrm{i}) .{ }^{*} \mathrm{Wq}\right)+\operatorname{sum}\left(\mathrm{g} 3313 \mathrm{~b}(\mathrm{i}) .{ }^{*} \mathrm{Wq}\right)+\operatorname{sum}\left(\mathrm{g} 1333 \mathrm{a}(\mathrm{i}) .{ }^{*} \mathrm{Wq}\right)+\operatorname{sum}(\mathrm{g} 13\right.\right.$

$33 \mathrm{~b}(\mathrm{i}) \cdot * \mathrm{Wq})))) /(8 * \mathrm{pi})$;

S3132

$\left(\left(\mathrm{L}(1,7) *\left(\operatorname{sum}\left(\mathrm{g} 3111 \mathrm{a}(\mathrm{i}) .{ }^{*} \mathrm{Wq}\right)+\operatorname{sum}\left(\mathrm{g} 3111 \mathrm{~b}(\mathrm{i}) .{ }^{*} \mathrm{Wq}\right)+\operatorname{sum}\left(\mathrm{g} 1131 \mathrm{a}(\mathrm{i}) .{ }^{*} \mathrm{Wq}\right)+\operatorname{sum}(\mathrm{g} 1\right.\right.\right.$ $\left.\left.\left.131 \mathrm{~b}(\mathrm{i}) \cdot{ }^{*} \mathrm{Wq}\right)\right)\right)$

$\left(\mathrm{L}(6,7) *\left(\operatorname{sum}\left(\mathrm{g} 3112 \mathrm{a}(\mathrm{i}) .{ }^{*} \mathrm{Wq}\right)+\operatorname{sum}\left(\mathrm{g} 3112 \mathrm{~b}(\mathrm{i}) \cdot{ }^{*} \mathrm{Wq}\right)+\operatorname{sum}\left(\mathrm{g} 1132 \mathrm{a}(\mathrm{i}) .{ }^{*} \mathrm{Wq}\right)+\operatorname{sum}(\mathrm{g} 11\right.\right.$ $\left.\left.\left.32 \mathrm{~b}(\mathrm{i}) *{ }^{*} \mathrm{Wq}\right)\right)\right)$

$\left(\mathrm{L}(8,7) *\left(\operatorname{sum}\left(\mathrm{g} 3113 \mathrm{a}(\mathrm{i}) .{ }^{*} \mathrm{Wq}\right)+\operatorname{sum}\left(\mathrm{g} 3113 \mathrm{~b}(\mathrm{i}) .{ }^{*} \mathrm{Wq}\right)+\operatorname{sum}\left(\mathrm{g} 1133 \mathrm{a}(\mathrm{i}) .{ }^{*} \mathrm{Wq}\right)+\operatorname{sum}(\mathrm{g} 11\right.\right.$ $33 \mathrm{~b}(\mathrm{i}) . * \mathrm{Wq}))$ )

$\left(\mathrm{L}(9,7) *\left(\operatorname{sum}\left(\mathrm{g} 3211 \mathrm{a}(\mathrm{i}) .{ }^{*} \mathrm{Wq}\right)+\operatorname{sum}\left(\mathrm{g} 3211 \mathrm{~b}(\mathrm{i}) .{ }^{*} \mathrm{Wq}\right)+\operatorname{sum}\left(\mathrm{g} 1231 \mathrm{a}(\mathrm{i}) .{ }^{*} \mathrm{Wq}\right)+\operatorname{sum}(\mathrm{g} 12\right.\right.$ $31 \mathrm{~b}(\mathrm{i}) . * \mathrm{Wq}))$ )

$\left(\mathrm{L}(2,7) *\left(\operatorname{sum}\left(\mathrm{g} 3212 \mathrm{a}(\mathrm{i}) .{ }^{*} \mathrm{Wq}\right)+\operatorname{sum}\left(\mathrm{g} 3212 \mathrm{~b}(\mathrm{i}) .{ }^{*} \mathrm{Wq}\right)+\operatorname{sum}\left(\mathrm{g} 1232 \mathrm{a}(\mathrm{i}) .{ }^{*} \mathrm{Wq}\right)+\operatorname{sum}(\mathrm{g} 12\right.\right.$ $32 \mathrm{~b}(\mathrm{i}) . * \mathrm{Wq}))$ )

$\left(\mathrm{L}(4,7) *\left(\operatorname{sum}\left(\mathrm{g} 3213 \mathrm{a}(\mathrm{i}) .{ }^{*} \mathrm{Wq}\right)+\operatorname{sum}\left(\mathrm{g} 3213 \mathrm{~b}(\mathrm{i}) .{ }^{*} \mathrm{Wq}\right)+\operatorname{sum}\left(\mathrm{g} 1233 \mathrm{a}(\mathrm{i}) .{ }^{*} \mathrm{Wq}\right)+\operatorname{sum}(\mathrm{g} 12\right.\right.$ $33 \mathrm{~b}(\mathrm{i}) . * \mathrm{Wq})))$

$\left(\mathrm{L}(5,7) *\left(\operatorname{sum}\left(\mathrm{g} 3311 \mathrm{a}(\mathrm{i}) .{ }^{*} \mathrm{Wq}\right)+\operatorname{sum}\left(\mathrm{g} 3311 \mathrm{~b}(\mathrm{i}) .{ }^{*} \mathrm{Wq}\right)+\operatorname{sum}(\mathrm{g} 1331 \mathrm{a}(\mathrm{i}) . * \mathrm{Wq})+\operatorname{sum}(\mathrm{g} 13\right.\right.$ $31 \mathrm{~b}(\mathrm{i}) . * \mathrm{Wq}))$ )

$\left(\mathrm{L}(7,7) *\left(\operatorname{sum}\left(\mathrm{g} 3312 \mathrm{a}(\mathrm{i}) .{ }^{*} \mathrm{Wq}\right)+\operatorname{sum}\left(\mathrm{g} 3312 \mathrm{~b}(\mathrm{i}) .{ }^{*} \mathrm{Wq}\right)+\operatorname{sum}\left(\mathrm{g} 1332 \mathrm{a}(\mathrm{i}) .{ }^{*} \mathrm{Wq}\right)+\operatorname{sum}(\mathrm{g} 13\right.\right.$ $32 \mathrm{~b}(\mathrm{i}) . * \mathrm{Wq})))$

$\left(\mathrm{L}(3,7) *\left(\operatorname{sum}(\mathrm{g} 3313 \mathrm{a}(\mathrm{i}) . * \mathrm{Wq})+\operatorname{sum}\left(\mathrm{g} 3313 \mathrm{~b}(\mathrm{i}) \cdot{ }^{*} \mathrm{Wq}\right)+\operatorname{sum}\left(\mathrm{g} 1333 \mathrm{a}(\mathrm{i}) \cdot{ }^{*} \mathrm{Wq}\right)+\operatorname{sum}(\mathrm{g} 13\right.\right.$ $33 \mathrm{~b}(\mathrm{i}) *$ Wq $)))) /(8 * \mathrm{pi})$;

S3113

$\left(\left(\mathrm{L}(1,8) *\left(\operatorname{sum}\left(\mathrm{g} 3111 \mathrm{a}(\mathrm{i}) .{ }^{*} \mathrm{Wq}\right)+\operatorname{sum}\left(\mathrm{g} 3111 \mathrm{~b}(\mathrm{i}){ }^{*} \mathrm{Wq}\right)+\operatorname{sum}\left(\mathrm{g} 1131 \mathrm{a}(\mathrm{i}) .{ }^{*} \mathrm{Wq}\right)+\operatorname{sum}(\mathrm{g} 1\right.\right.\right.$ $131 \mathrm{~b}(\mathrm{i}) \cdot * \mathrm{Wq})))$

$\left(\mathrm{L}(6,8) *\left(\operatorname{sum}\left(\mathrm{g} 3112 \mathrm{a}(\mathrm{i}) .{ }^{*} \mathrm{Wq}\right)+\operatorname{sum}\left(\mathrm{g} 3112 \mathrm{~b}(\mathrm{i}) .{ }^{*} \mathrm{Wq}\right)+\operatorname{sum}\left(\mathrm{g} 1132 \mathrm{a}(\mathrm{i}) .{ }^{*} \mathrm{Wq}\right)+\operatorname{sum}(\mathrm{g} 11\right.\right.$ $32 \mathrm{~b}(\mathrm{i}) . * \mathrm{Wq}))$ )

$\left(\mathrm{L}(8,8) *\left(\operatorname{sum}\left(\mathrm{g} 3113 \mathrm{a}(\mathrm{i}) .{ }^{*} \mathrm{Wq}\right)+\operatorname{sum}\left(\mathrm{g} 3113 \mathrm{~b}(\mathrm{i}) \cdot{ }^{*} \mathrm{Wq}\right)+\operatorname{sum}\left(\mathrm{g} 1133 \mathrm{a}(\mathrm{i}) .{ }^{*} \mathrm{Wq}\right)+\operatorname{sum}(\mathrm{g} 11\right.\right.$ $33 \mathrm{~b}(\mathrm{i}) . * \mathrm{Wq})))$

$\left(\mathrm{L}(9,8) *\left(\operatorname{sum}\left(\mathrm{g} 3211 \mathrm{a}(\mathrm{i}) .{ }^{*} \mathrm{Wq}\right)+\operatorname{sum}\left(\mathrm{g} 3211 \mathrm{~b}(\mathrm{i}) \cdot{ }^{*} \mathrm{Wq}\right)+\operatorname{sum}\left(\mathrm{g} 1231 \mathrm{a}(\mathrm{i}) .{ }^{*} \mathrm{Wq}\right)+\operatorname{sum}(\mathrm{g} 12\right.\right.$ $31 \mathrm{~b}(\mathrm{i}) . * \mathrm{Wq}))$ )

$\left(\mathrm{L}(2,8) *\left(\operatorname{sum}\left(\mathrm{g} 3212 \mathrm{a}(\mathrm{i}) \cdot{ }^{*} \mathrm{Wq}\right)+\operatorname{sum}\left(\mathrm{g} 3212 \mathrm{~b}(\mathrm{i}) .{ }^{*} \mathrm{Wq}\right)+\operatorname{sum}\left(\mathrm{g} 1232 \mathrm{a}(\mathrm{i}) .{ }^{*} \mathrm{Wq}\right)+\operatorname{sum}(\mathrm{g} 12\right.\right.$ $\left.\left.\left.32 \mathrm{~b}(\mathrm{i}) *{ }^{*} \mathrm{Wq}\right)\right)\right)$

$\left(\mathrm{L}(4,8) *\left(\operatorname{sum}\left(\mathrm{g} 3213 \mathrm{a}(\mathrm{i}) .{ }^{*} \mathrm{Wq}\right)+\operatorname{sum}\left(\mathrm{g} 3213 \mathrm{~b}(\mathrm{i}) .{ }^{*} \mathrm{Wq}\right)+\operatorname{sum}\left(\mathrm{g} 1233 \mathrm{a}(\mathrm{i}) .{ }^{*} \mathrm{Wq}\right)+\operatorname{sum}(\mathrm{g} 12\right.\right.$ $33 \mathrm{~b}(\mathrm{i}) . * \mathrm{Wq}))$ )

$\left(\mathrm{L}(5,8) *\left(\operatorname{sum}\left(\mathrm{g} 3311 \mathrm{a}(\mathrm{i}) .{ }^{*} \mathrm{Wq}\right)+\operatorname{sum}\left(\mathrm{g} 3311 \mathrm{~b}(\mathrm{i}) .{ }^{*} \mathrm{Wq}\right)+\operatorname{sum}\left(\mathrm{g} 1331 \mathrm{a}(\mathrm{i}) .{ }^{*} \mathrm{Wq}\right)+\operatorname{sum}(\mathrm{g} 13\right.\right.$ $31 \mathrm{~b}(\mathrm{i}) . * \mathrm{Wq})))$ $\left(\mathrm{L}(7,8) *\left(\operatorname{sum}\left(\mathrm{g} 3312 \mathrm{a}(\mathrm{i}) .{ }^{*} \mathrm{Wq}\right)+\operatorname{sum}\left(\mathrm{g} 3312 \mathrm{~b}(\mathrm{i}) \cdot{ }^{*} \mathrm{Wq}\right)+\operatorname{sum}\left(\mathrm{g} 1332 \mathrm{a}(\mathrm{i}) \cdot{ }^{*} \mathrm{Wq}\right)+\operatorname{sum}(\mathrm{g} 13\right.\right.$ $32 \mathrm{~b}(\mathrm{i}) . * \mathrm{Wq}))$ ) $\left(\mathrm{L}(3,8) *\left(\operatorname{sum}\left(\mathrm{g} 3313 \mathrm{a}(\mathrm{i}) .{ }^{*} \mathrm{Wq}\right)+\operatorname{sum}\left(\mathrm{g} 3313 \mathrm{~b}(\mathrm{i}) .{ }^{*} \mathrm{Wq}\right)+\operatorname{sum}\left(\mathrm{g} 1333 \mathrm{a}(\mathrm{i}) .{ }^{*} \mathrm{Wq}\right)+\operatorname{sum}(\mathrm{g} 13\right.\right.$ $33 \mathrm{~b}(\mathrm{i}) *$ Wq $)))) /(8 * \mathrm{pi})$; 
S3121

$\left(\left(\mathrm{L}(1,9) *\left(\operatorname{sum}\left(\mathrm{g} 3111 \mathrm{a}(\mathrm{i}) .{ }^{*} \mathrm{Wq}\right)+\operatorname{sum}\left(\mathrm{g} 3111 \mathrm{~b}(\mathrm{i}) .{ }^{*} \mathrm{Wq}\right)+\operatorname{sum}(\mathrm{g} 1131 \mathrm{a}(\mathrm{i}) . * \mathrm{Wq})+\operatorname{sum}(\mathrm{g} 1\right.\right.\right.$ $\left.\left.\left.131 \mathrm{~b}(\mathrm{i}) \cdot{ }^{*} \mathrm{Wq}\right)\right)\right)$

$\left(\mathrm{L}(6,9) *\left(\operatorname{sum}\left(\mathrm{g} 3112 \mathrm{a}(\mathrm{i}) .{ }^{*} \mathrm{Wq}\right)+\operatorname{sum}\left(\mathrm{g} 3112 \mathrm{~b}(\mathrm{i}) \cdot{ }^{*} \mathrm{Wq}\right)+\operatorname{sum}\left(\mathrm{g} 1132 \mathrm{a}(\mathrm{i}) .{ }^{*} \mathrm{Wq}\right)+\operatorname{sum}(\mathrm{g} 11\right.\right.$ $32 \mathrm{~b}(\mathrm{i}) . * \mathrm{Wq}))$

$\left(\mathrm{L}(8,9) *\left(\operatorname{sum}\left(\mathrm{g} 3113 \mathrm{a}(\mathrm{i}) .{ }^{*} \mathrm{Wq}\right)+\operatorname{sum}\left(\mathrm{g} 3113 \mathrm{~b}(\mathrm{i}) .{ }^{*} \mathrm{Wq}\right)+\operatorname{sum}\left(\mathrm{g} 1133 \mathrm{a}(\mathrm{i}) .{ }^{*} \mathrm{Wq}\right)+\operatorname{sum}(\mathrm{g} 11\right.\right.$ $\left.\left.\left.33 \mathrm{~b}(\mathrm{i}) \cdot{ }^{*} \mathrm{Wq}\right)\right)\right)$

$\left(\mathrm{L}(9,9) *\left(\operatorname{sum}\left(\mathrm{g} 3211 \mathrm{a}(\mathrm{i}) .{ }^{*} \mathrm{Wq}\right)+\operatorname{sum}\left(\mathrm{g} 3211 \mathrm{~b}(\mathrm{i}) .{ }^{*} \mathrm{Wq}\right)+\operatorname{sum}\left(\mathrm{g} 1231 \mathrm{a}(\mathrm{i}) .{ }^{*} \mathrm{Wq}\right)+\operatorname{sum}(\mathrm{g} 12\right.\right.$ $31 \mathrm{~b}(\mathrm{i}) . * \mathrm{Wq}))$

$\left(\mathrm{L}(2,9) *\left(\operatorname{sum}\left(\mathrm{g} 3212 \mathrm{a}(\mathrm{i}) .{ }^{*} \mathrm{Wq}\right)+\operatorname{sum}\left(\mathrm{g} 3212 \mathrm{~b}(\mathrm{i}) .{ }^{*} \mathrm{Wq}\right)+\operatorname{sum}(\mathrm{g} 1232 \mathrm{a}(\mathrm{i}) . * \mathrm{Wq})+\operatorname{sum}(\mathrm{g} 12\right.\right.$ $32 \mathrm{~b}(\mathrm{i}) . * \mathrm{Wq})))$

$\left(\mathrm{L}(4,9) *\left(\operatorname{sum}(\mathrm{g} 3213 \mathrm{a}(\mathrm{i}) . * \mathrm{Wq})+\operatorname{sum}\left(\mathrm{g} 3213 \mathrm{~b}(\mathrm{i}) \cdot{ }^{*} \mathrm{Wq}\right)+\operatorname{sum}\left(\mathrm{g} 1233 \mathrm{a}(\mathrm{i}) \cdot{ }^{*} \mathrm{Wq}\right)+\operatorname{sum}(\mathrm{g} 12\right.\right.$ $\left.\left.33 \mathrm{~b}(\mathrm{i}) \cdot{ }^{*} \mathrm{Wq}\right)\right)$

$\left(\mathrm{L}(5,9) *\left(\operatorname{sum}\left(\mathrm{g} 3311 \mathrm{a}(\mathrm{i}) .{ }^{*} \mathrm{Wq}\right)+\operatorname{sum}\left(\mathrm{g} 3311 \mathrm{~b}(\mathrm{i}) .{ }^{*} \mathrm{Wq}\right)+\operatorname{sum}(\mathrm{g} 1331 \mathrm{a}(\mathrm{i}) . * \mathrm{Wq})+\operatorname{sum}(\mathrm{g} 13\right.\right.$ $31 \mathrm{~b}(\mathrm{i}) . * \mathrm{Wq})))$

$\left(\mathrm{L}(7,9) *\left(\operatorname{sum}(\mathrm{g} 3312 \mathrm{a}(\mathrm{i}) . * \mathrm{Wq})+\operatorname{sum}\left(\mathrm{g} 3312 \mathrm{~b}(\mathrm{i}) \cdot{ }^{*} \mathrm{Wq}\right)+\operatorname{sum}\left(\mathrm{g} 1332 \mathrm{a}(\mathrm{i}) \cdot{ }^{*} \mathrm{Wq}\right)+\operatorname{sum}(\mathrm{g} 13\right.\right.$ $32 \mathrm{~b}(\mathrm{i}) . * \mathrm{Wq}))$ )

$\left(\mathrm{L}(3,9) *\left(\operatorname{sum}\left(\mathrm{g} 3313 \mathrm{a}(\mathrm{i}) .{ }^{*} \mathrm{Wq}\right)+\operatorname{sum}\left(\mathrm{g} 3313 \mathrm{~b}(\mathrm{i}) .{ }^{*} \mathrm{Wq}\right)+\operatorname{sum}\left(\mathrm{g} 1333 \mathrm{a}(\mathrm{i}) .{ }^{*} \mathrm{Wq}\right)+\operatorname{sum}(\mathrm{g} 13\right.\right.$

$\left.\left.\left.\left.33 \mathrm{~b}(\mathrm{i}){ }^{*} \mathrm{Wq}\right)\right)\right)\right) /(8 * \mathrm{pi})$;

$\mathrm{S} 1211$

$\left(\left(\mathrm{L}(1,1) *\left(\operatorname{sum}\left(\mathrm{g} 1121 \mathrm{a}(\mathrm{i}) .{ }^{*} \mathrm{Wq}\right)+\operatorname{sum}\left(\mathrm{g} 1121 \mathrm{~b}(\mathrm{i}) .{ }^{*} \mathrm{Wq}\right)+\operatorname{sum}(\mathrm{g} 2111 \mathrm{a}(\mathrm{i}) . * \mathrm{Wq})+\operatorname{sum}(\mathrm{g} 2\right.\right.\right.$ $\left.\left.\left.111 \mathrm{~b}(\mathrm{i}) *{ }^{*} \mathrm{Wq}\right)\right)\right)$

$\left(\mathrm{L}(6,1) *\left(\operatorname{sum}\left(\mathrm{g} 1122 \mathrm{a}(\mathrm{i}) .{ }^{*} \mathrm{Wq}\right)+\operatorname{sum}\left(\mathrm{g} 1122 \mathrm{~b}(\mathrm{i}) \cdot{ }^{*} \mathrm{Wq}\right)+\operatorname{sum}\left(\mathrm{g} 2112 \mathrm{a}(\mathrm{i}) .{ }^{*} \mathrm{Wq}\right)+\operatorname{sum}(\mathrm{g} 21\right.\right.$ $12 \mathrm{~b}(\mathrm{i}) . * \mathrm{Wq})))$

$\left(\mathrm{L}(8,1) *\left(\operatorname{sum}\left(\mathrm{g} 1123 \mathrm{a}(\mathrm{i}) .{ }^{*} \mathrm{Wq}\right)+\operatorname{sum}\left(\mathrm{g} 1123 \mathrm{~b}(\mathrm{i}) .{ }^{*} \mathrm{Wq}\right)+\operatorname{sum}\left(\mathrm{g} 2113 \mathrm{a}(\mathrm{i}) .{ }^{*} \mathrm{Wq}\right)+\operatorname{sum}(\mathrm{g} 21\right.\right.$ $13 \mathrm{~b}(\mathrm{i}) . * \mathrm{Wq}))$

$\left(\mathrm{L}(9,1) *\left(\operatorname{sum}\left(\mathrm{g} 1221 \mathrm{a}(\mathrm{i}) .{ }^{*} \mathrm{Wq}\right)+\operatorname{sum}\left(\mathrm{g} 1221 \mathrm{~b}(\mathrm{i}) .{ }^{*} \mathrm{Wq}\right)+\operatorname{sum}\left(\mathrm{g} 2211 \mathrm{a}(\mathrm{i}) .{ }^{*} \mathrm{Wq}\right)+\operatorname{sum}(\mathrm{g} 22\right.\right.$ $11 \mathrm{~b}(\mathrm{i}) . * \mathrm{Wq})))$

$\left(\mathrm{L}(2,1) *\left(\operatorname{sum}\left(\mathrm{g} 1222 \mathrm{a}(\mathrm{i}) .{ }^{*} \mathrm{Wq}\right)+\operatorname{sum}\left(\mathrm{g} 1222 \mathrm{~b}(\mathrm{i}) .{ }^{*} \mathrm{Wq}\right)+\operatorname{sum}\left(\mathrm{g} 2212 \mathrm{a}(\mathrm{i}) .{ }^{*} \mathrm{Wq}\right)+\operatorname{sum}(\mathrm{g} 22\right.\right.$ $12 \mathrm{~b}(\mathrm{i}) . * \mathrm{Wq})))$

$\left(\mathrm{L}(4,1) *\left(\operatorname{sum}\left(\mathrm{g} 1223 \mathrm{a}(\mathrm{i}) .{ }^{*} \mathrm{Wq}\right)+\operatorname{sum}\left(\mathrm{g} 1223 \mathrm{~b}(\mathrm{i}) .{ }^{*} \mathrm{Wq}\right)+\operatorname{sum}\left(\mathrm{g} 2213 \mathrm{a}(\mathrm{i}) .{ }^{*} \mathrm{Wq}\right)+\operatorname{sum}(\mathrm{g} 22\right.\right.$ $13 \mathrm{~b}(\mathrm{i}) . * \mathrm{Wq}))$

$\left(\mathrm{L}(5,1) *\left(\operatorname{sum}\left(\mathrm{g} 1321 \mathrm{a}(\mathrm{i}) .{ }^{*} \mathrm{Wq}\right)+\operatorname{sum}\left(\mathrm{g} 1321 \mathrm{~b}(\mathrm{i}) .{ }^{*} \mathrm{Wq}\right)+\operatorname{sum}\left(\mathrm{g} 2311 \mathrm{a}(\mathrm{i}) .{ }^{*} \mathrm{Wq}\right)+\operatorname{sum}(\mathrm{g} 23\right.\right.$ $11 \mathrm{~b}(\mathrm{i}) . * \mathrm{Wq})))$

$\left(\mathrm{L}(7,1) *\left(\operatorname{sum}\left(\mathrm{g} 1322 \mathrm{a}(\mathrm{i}) .{ }^{*} \mathrm{Wq}\right)+\operatorname{sum}\left(\mathrm{g} 1322 \mathrm{~b}(\mathrm{i}) \cdot{ }^{*} \mathrm{Wq}\right)+\operatorname{sum}(\mathrm{g} 2312 \mathrm{a}(\mathrm{i}) . * \mathrm{Wq})+\operatorname{sum}(\mathrm{g} 23\right.\right.$ $12 \mathrm{~b}(\mathrm{i}) . * \mathrm{Wq}))$

$\left(\mathrm{L}(3,1) *\left(\operatorname{sum}\left(\mathrm{g} 1323 \mathrm{a}(\mathrm{i}) .{ }^{*} \mathrm{Wq}\right)+\operatorname{sum}\left(\mathrm{g} 1323 \mathrm{~b}(\mathrm{i}) \cdot{ }^{*} \mathrm{Wq}\right)+\operatorname{sum}\left(\mathrm{g} 2313 \mathrm{a}(\mathrm{i}) \cdot{ }^{*} \mathrm{Wq}\right)+\operatorname{sum}(\mathrm{g} 23\right.\right.$ $\left.\left.\left.\left.13 \mathrm{~b}(\mathrm{i}) \cdot{ }^{*} \mathrm{Wq}\right)\right)\right)\right) /(8 * \mathrm{pi})$;

$\mathrm{S} 1222$

$\left(\left(\mathrm{L}(1,2) *\left(\operatorname{sum}\left(\mathrm{g} 1121 \mathrm{a}(\mathrm{i}) .{ }^{*} \mathrm{Wq}\right)+\operatorname{sum}\left(\mathrm{g} 1121 \mathrm{~b}(\mathrm{i}) .{ }^{*} \mathrm{Wq}\right)+\operatorname{sum}\left(\mathrm{g} 2111 \mathrm{a}(\mathrm{i}) .{ }^{*} \mathrm{Wq}\right)+\operatorname{sum}(\mathrm{g} 2\right.\right.\right.$ $111 \mathrm{~b}(\mathrm{i}) * \mathrm{Wq})))$

$\left(\mathrm{L}(6,2) *\left(\operatorname{sum}\left(\mathrm{g} 1122 \mathrm{a}(\mathrm{i}) .{ }^{*} \mathrm{Wq}\right)+\operatorname{sum}\left(\mathrm{g} 1122 \mathrm{~b}(\mathrm{i}) .{ }^{*} \mathrm{Wq}\right)+\operatorname{sum}(\mathrm{g} 2112 \mathrm{a}(\mathrm{i}) . * \mathrm{Wq})+\operatorname{sum}(\mathrm{g} 21\right.\right.$ 
$12 \mathrm{~b}(\mathrm{i}) . * \mathrm{Wq})))$

$\left(\mathrm{L}(8,2) *\left(\operatorname{sum}\left(\mathrm{g} 1123 \mathrm{a}(\mathrm{i}) .{ }^{*} \mathrm{Wq}\right)+\operatorname{sum}\left(\mathrm{g} 1123 \mathrm{~b}(\mathrm{i}) .{ }^{*} \mathrm{Wq}\right)+\operatorname{sum}\left(\mathrm{g} 2113 \mathrm{a}(\mathrm{i}) .{ }^{*} \mathrm{Wq}\right)+\operatorname{sum}(\mathrm{g} 21\right.\right.$

$13 \mathrm{~b}(\mathrm{i}) . * \mathrm{Wq}))$ )

$\left(\mathrm{L}(9,2) *\left(\operatorname{sum}(\mathrm{g} 1221 \mathrm{a}(\mathrm{i}) . * \mathrm{Wq})+\operatorname{sum}\left(\mathrm{g} 1221 \mathrm{~b}(\mathrm{i}) \cdot{ }^{*} \mathrm{Wq}\right)+\operatorname{sum}\left(\mathrm{g} 2211 \mathrm{a}(\mathrm{i}) \cdot{ }^{*} \mathrm{Wq}\right)+\operatorname{sum}(\mathrm{g} 22\right.\right.$

$11 \mathrm{~b}(\mathrm{i}) . * \mathrm{Wq})))$

$\left(\mathrm{L}(2,2) *\left(\operatorname{sum}(\mathrm{g} 1222 \mathrm{a}(\mathrm{i}) . * \mathrm{Wq})+\operatorname{sum}\left(\mathrm{g} 1222 \mathrm{~b}(\mathrm{i}) \cdot{ }^{*} \mathrm{Wq}\right)+\operatorname{sum}\left(\mathrm{g} 2212 \mathrm{a}(\mathrm{i}) \cdot{ }^{*} \mathrm{Wq}\right)+\operatorname{sum}(\mathrm{g} 22\right.\right.$

$12 \mathrm{~b}(\mathrm{i}) . * \mathrm{Wq})))$

$\left(\mathrm{L}(4,2) *\left(\operatorname{sum}\left(\mathrm{g} 1223 \mathrm{a}(\mathrm{i}) .{ }^{*} \mathrm{Wq}\right)+\operatorname{sum}\left(\mathrm{g} 1223 \mathrm{~b}(\mathrm{i}) .{ }^{*} \mathrm{Wq}\right)+\operatorname{sum}\left(\mathrm{g} 2213 \mathrm{a}(\mathrm{i}) .{ }^{*} \mathrm{Wq}\right)+\operatorname{sum}(\mathrm{g} 22\right.\right.$

$13 \mathrm{~b}(\mathrm{i}) . * \mathrm{Wq}))$

$\left(\mathrm{L}(5,2) *\left(\operatorname{sum}\left(\mathrm{g} 1321 \mathrm{a}(\mathrm{i}) .{ }^{*} \mathrm{Wq}\right)+\operatorname{sum}\left(\mathrm{g} 1321 \mathrm{~b}(\mathrm{i}) .{ }^{*} \mathrm{Wq}\right)+\operatorname{sum}\left(\mathrm{g} 2311 \mathrm{a}(\mathrm{i}) .{ }^{*} \mathrm{Wq}\right)+\operatorname{sum}(\mathrm{g} 23\right.\right.$

$11 \mathrm{~b}(\mathrm{i}) . * \mathrm{Wq})))$

$\left(\mathrm{L}(7,2) *\left(\operatorname{sum}\left(\mathrm{g} 1322 \mathrm{a}(\mathrm{i}) .{ }^{*} \mathrm{Wq}\right)+\operatorname{sum}\left(\mathrm{g} 1322 \mathrm{~b}(\mathrm{i}) \cdot{ }^{*} \mathrm{Wq}\right)+\operatorname{sum}(\mathrm{g} 2312 \mathrm{a}(\mathrm{i}) . * \mathrm{Wq})+\operatorname{sum}(\mathrm{g} 23\right.\right.$

$\left.\left.\left.12 \mathrm{~b}(\mathrm{i}) \cdot{ }^{*} \mathrm{Wq}\right)\right)\right)$

$\left(\mathrm{L}(3,2) *\left(\operatorname{sum}\left(\mathrm{g} 1323 \mathrm{a}(\mathrm{i}) .{ }^{*} \mathrm{Wq}\right)+\operatorname{sum}\left(\mathrm{g} 1323 \mathrm{~b}(\mathrm{i}) \cdot{ }^{*} \mathrm{Wq}\right)+\operatorname{sum}\left(\mathrm{g} 2313 \mathrm{a}(\mathrm{i}) .{ }^{*} \mathrm{Wq}\right)+\operatorname{sum}(\mathrm{g} 23\right.\right.$

$\left.\left.\left.\left.13 \mathrm{~b}(\mathrm{i}){ }^{*} \mathrm{Wq}\right)\right)\right)\right) /(8 * \mathrm{pi})$;

$\mathrm{S} 1233$

$\left(\left(\mathrm{L}(1,3) *\left(\operatorname{sum}\left(\mathrm{g} 1121 \mathrm{a}(\mathrm{i}) .{ }^{*} \mathrm{Wq}\right)+\operatorname{sum}\left(\mathrm{g} 1121 \mathrm{~b}(\mathrm{i}) \cdot{ }^{*} \mathrm{Wq}\right)+\operatorname{sum}\left(\mathrm{g} 2111 \mathrm{a}(\mathrm{i}) .{ }^{*} \mathrm{Wq}\right)+\operatorname{sum}(\mathrm{g} 2\right.\right.\right.$ $\left.\left.\left.111 \mathrm{~b}(\mathrm{i}) \cdot{ }^{*} \mathrm{Wq}\right)\right)\right)$

$\left(\mathrm{L}(6,3) *\left(\operatorname{sum}\left(\mathrm{g} 1122 \mathrm{a}(\mathrm{i}) .{ }^{*} \mathrm{Wq}\right)+\operatorname{sum}\left(\mathrm{g} 1122 \mathrm{~b}(\mathrm{i}) .{ }^{*} \mathrm{Wq}\right)+\operatorname{sum}\left(\mathrm{g} 2112 \mathrm{a}(\mathrm{i}) .{ }^{*} \mathrm{Wq}\right)+\operatorname{sum}(\mathrm{g} 21\right.\right.$

$12 \mathrm{~b}(\mathrm{i}) . * \mathrm{Wq})))$

$\left(\mathrm{L}(8,3) *\left(\operatorname{sum}\left(\mathrm{g} 1123 \mathrm{a}(\mathrm{i}) .{ }^{*} \mathrm{Wq}\right)+\operatorname{sum}\left(\mathrm{g} 1123 \mathrm{~b}(\mathrm{i}) .{ }^{*} \mathrm{Wq}\right)+\operatorname{sum}\left(\mathrm{g} 2113 \mathrm{a}(\mathrm{i}) .{ }^{*} \mathrm{Wq}\right)+\operatorname{sum}(\mathrm{g} 21\right.\right.$

$13 \mathrm{~b}(\mathrm{i}) . * \mathrm{Wq})))$

$\left(\mathrm{L}(9,3) *\left(\operatorname{sum}(\mathrm{g} 1221 \mathrm{a}(\mathrm{i}) . * \mathrm{Wq})+\operatorname{sum}\left(\mathrm{g} 1221 \mathrm{~b}(\mathrm{i}) \cdot{ }^{*} \mathrm{Wq}\right)+\operatorname{sum}(\mathrm{g} 2211 \mathrm{a}(\mathrm{i}) . * \mathrm{Wq})+\operatorname{sum}(\mathrm{g} 22\right.\right.$

$11 \mathrm{~b}(\mathrm{i}) . * \mathrm{Wq})))$

$\left(\mathrm{L}(2,3) *\left(\operatorname{sum}\left(\mathrm{g} 1222 \mathrm{a}(\mathrm{i}) \cdot{ }^{*} \mathrm{Wq}\right)+\operatorname{sum}\left(\mathrm{g} 1222 \mathrm{~b}(\mathrm{i}) \cdot{ }^{*} \mathrm{Wq}\right)+\operatorname{sum}\left(\mathrm{g} 2212 \mathrm{a}(\mathrm{i}) \cdot{ }^{*} \mathrm{Wq}\right)+\operatorname{sum}(\mathrm{g} 22\right.\right.$

$12 \mathrm{~b}(\mathrm{i}) . * \mathrm{Wq})))$

$\left(\mathrm{L}(4,3) *\left(\operatorname{sum}\left(\mathrm{g} 1223 \mathrm{a}(\mathrm{i}) .{ }^{*} \mathrm{Wq}\right)+\operatorname{sum}\left(\mathrm{g} 1223 \mathrm{~b}(\mathrm{i}) .{ }^{*} \mathrm{Wq}\right)+\operatorname{sum}\left(\mathrm{g} 2213 \mathrm{a}(\mathrm{i}) .{ }^{*} \mathrm{Wq}\right)+\operatorname{sum}(\mathrm{g} 22\right.\right.$

$13 \mathrm{~b}(\mathrm{i}) . * \mathrm{Wq}))$

$\left(\mathrm{L}(5,3) *\left(\operatorname{sum}\left(\mathrm{g} 1321 \mathrm{a}(\mathrm{i}) .{ }^{*} \mathrm{Wq}\right)+\operatorname{sum}\left(\mathrm{g} 1321 \mathrm{~b}(\mathrm{i}) .{ }^{*} \mathrm{Wq}\right)+\operatorname{sum}(\mathrm{g} 2311 \mathrm{a}(\mathrm{i}) . * \mathrm{Wq})+\operatorname{sum}(\mathrm{g} 23\right.\right.$

$11 \mathrm{~b}(\mathrm{i}) . * \mathrm{Wq})))$

$\left(\mathrm{L}(7,3) *\left(\operatorname{sum}\left(\mathrm{g} 1322 \mathrm{a}(\mathrm{i}) .{ }^{*} \mathrm{Wq}\right)+\operatorname{sum}\left(\mathrm{g} 1322 \mathrm{~b}(\mathrm{i}) .{ }^{*} \mathrm{Wq}\right)+\operatorname{sum}\left(\mathrm{g} 2312 \mathrm{a}(\mathrm{i}) .{ }^{*} \mathrm{Wq}\right)+\operatorname{sum}(\mathrm{g} 23\right.\right.$

$12 \mathrm{~b}(\mathrm{i}) . * \mathrm{Wq})))$

$\left(\mathrm{L}(3,3) *\left(\operatorname{sum}\left(\mathrm{g} 1323 \mathrm{a}(\mathrm{i}) .{ }^{*} \mathrm{Wq}\right)+\operatorname{sum}\left(\mathrm{g} 1323 \mathrm{~b}(\mathrm{i}) .{ }^{*} \mathrm{Wq}\right)+\operatorname{sum}\left(\mathrm{g} 2313 \mathrm{a}(\mathrm{i}) .{ }^{*} \mathrm{Wq}\right)+\operatorname{sum}(\mathrm{g} 23\right.\right.$

$13 \mathrm{~b}(\mathrm{i}) * \mathrm{Wq})))) /(8 * \mathrm{pi})$;

$\mathrm{S} 1223$

$\left(\left(\mathrm{L}(1,4) *\left(\operatorname{sum}(\mathrm{g} 1121 \mathrm{a}(\mathrm{i}) . * \mathrm{Wq})+\operatorname{sum}(\mathrm{g} 1121 \mathrm{~b}(\mathrm{i}) . * \mathrm{Wq})+\operatorname{sum}\left(\mathrm{g} 2111 \mathrm{a}(\mathrm{i}) .{ }^{*} \mathrm{Wq}\right)+\operatorname{sum}(\mathrm{g} 2\right.\right.\right.$ $111 \mathrm{~b}(\mathrm{i}) * \mathrm{Wq})))$

$\left(\mathrm{L}(6,4) *\left(\operatorname{sum}\left(\mathrm{g} 1122 \mathrm{a}(\mathrm{i}) .{ }^{*} \mathrm{Wq}\right)+\operatorname{sum}\left(\mathrm{g} 1122 \mathrm{~b}(\mathrm{i}) .{ }^{*} \mathrm{Wq}\right)+\operatorname{sum}\left(\mathrm{g} 2112 \mathrm{a}(\mathrm{i}) .{ }^{*} \mathrm{Wq}\right)+\operatorname{sum}(\mathrm{g} 21\right.\right.$

$12 \mathrm{~b}(\mathrm{i}) . * \mathrm{Wq})))$

$\left(\mathrm{L}(8,4) *\left(\operatorname{sum}\left(\mathrm{g} 1123 \mathrm{a}(\mathrm{i}) .{ }^{*} \mathrm{Wq}\right)+\operatorname{sum}\left(\mathrm{g} 1123 \mathrm{~b}(\mathrm{i}) .{ }^{*} \mathrm{Wq}\right)+\operatorname{sum}\left(\mathrm{g} 2113 \mathrm{a}(\mathrm{i}) .{ }^{*} \mathrm{Wq}\right)+\operatorname{sum}(\mathrm{g} 21\right.\right.$

$13 \mathrm{~b}(\mathrm{i}) . * \mathrm{Wq})))$

$\left(\mathrm{L}(9,4) *\left(\operatorname{sum}\left(\mathrm{g} 1221 \mathrm{a}(\mathrm{i}) .{ }^{*} \mathrm{Wq}\right)+\operatorname{sum}\left(\mathrm{g} 1221 \mathrm{~b}(\mathrm{i}) \cdot{ }^{*} \mathrm{Wq}\right)+\operatorname{sum}\left(\mathrm{g} 2211 \mathrm{a}(\mathrm{i}) \cdot{ }^{*} \mathrm{Wq}\right)+\operatorname{sum}(\mathrm{g} 22\right.\right.$

$\left.\left.\left.11 \mathrm{~b}(\mathrm{i}) \cdot{ }^{*} \mathrm{Wq}\right)\right)\right)$ 
$\left(\mathrm{L}(2,4) *\left(\operatorname{sum}\left(\mathrm{g} 1222 \mathrm{a}(\mathrm{i}) \cdot{ }^{*} \mathrm{Wq}\right)+\operatorname{sum}\left(\mathrm{g} 1222 \mathrm{~b}(\mathrm{i}) \cdot{ }^{*} \mathrm{Wq}\right)+\operatorname{sum}\left(\mathrm{g} 2212 \mathrm{a}(\mathrm{i}) \cdot{ }^{*} \mathrm{Wq}\right)+\operatorname{sum}(\mathrm{g} 22\right.\right.$ $12 \mathrm{~b}(\mathrm{i}) . * \mathrm{Wq}))$ ) $\left(\mathrm{L}(4,4) *\left(\operatorname{sum}(\mathrm{g} 1223 \mathrm{a}(\mathrm{i}) . * \mathrm{Wq})+\operatorname{sum}\left(\mathrm{g} 1223 \mathrm{~b}(\mathrm{i}) \cdot{ }^{*} \mathrm{Wq}\right)+\operatorname{sum}\left(\mathrm{g} 2213 \mathrm{a}(\mathrm{i}) \cdot{ }^{*} \mathrm{Wq}\right)+\operatorname{sum}(\mathrm{g} 22\right.\right.$ $13 \mathrm{~b}(\mathrm{i}) . * \mathrm{Wq})))$ $\left(\mathrm{L}(5,4) *\left(\operatorname{sum}\left(\mathrm{g} 1321 \mathrm{a}(\mathrm{i}) .{ }^{*} \mathrm{Wq}\right)+\operatorname{sum}\left(\mathrm{g} 1321 \mathrm{~b}(\mathrm{i}) .{ }^{*} \mathrm{Wq}\right)+\operatorname{sum}\left(\mathrm{g} 2311 \mathrm{a}(\mathrm{i}) .{ }^{*} \mathrm{Wq}\right)+\operatorname{sum}(\mathrm{g} 23\right.\right.$ $11 \mathrm{~b}(\mathrm{i}) . * \mathrm{Wq})))$ $\left(\mathrm{L}(7,4) *\left(\operatorname{sum}\left(\mathrm{g} 1322 \mathrm{a}(\mathrm{i}) .{ }^{*} \mathrm{Wq}\right)+\operatorname{sum}\left(\mathrm{g} 1322 \mathrm{~b}(\mathrm{i}) .{ }^{*} \mathrm{Wq}\right)+\operatorname{sum}\left(\mathrm{g} 2312 \mathrm{a}(\mathrm{i}) .{ }^{*} \mathrm{Wq}\right)+\operatorname{sum}(\mathrm{g} 23\right.\right.$ $12 \mathrm{~b}(\mathrm{i}) . * \mathrm{Wq})))$ $\left(\mathrm{L}(3,4) *\left(\operatorname{sum}\left(\mathrm{g} 1323 \mathrm{a}(\mathrm{i}) .{ }^{*} \mathrm{Wq}\right)+\operatorname{sum}\left(\mathrm{g} 1323 \mathrm{~b}(\mathrm{i}) .{ }^{*} \mathrm{Wq}\right)+\operatorname{sum}\left(\mathrm{g} 2313 \mathrm{a}(\mathrm{i}) .{ }^{*} \mathrm{Wq}\right)+\operatorname{sum}(\mathrm{g} 23\right.\right.$ $\left.\left.\left.\left.13 \mathrm{~b}(\mathrm{i}){ }^{*} \mathrm{Wq}\right)\right)\right)\right) /(8 * \mathrm{pi})$;

S1231

$\left(\left(\mathrm{L}(1,5) *\left(\operatorname{sum}\left(\mathrm{g} 1121 \mathrm{a}(\mathrm{i}) .{ }^{*} \mathrm{Wq}\right)+\operatorname{sum}\left(\mathrm{g} 1121 \mathrm{~b}(\mathrm{i}) .{ }^{*} \mathrm{Wq}\right)+\operatorname{sum}\left(\mathrm{g} 2111 \mathrm{a}(\mathrm{i}) .{ }^{*} \mathrm{Wq}\right)+\operatorname{sum}(\mathrm{g} 2\right.\right.\right.$ $\left.\left.\left.111 \mathrm{~b}(\mathrm{i}) \cdot{ }^{*} \mathrm{Wq}\right)\right)\right)$ $\left(\mathrm{L}(6,5) *\left(\operatorname{sum}\left(\mathrm{g} 1122 \mathrm{a}(\mathrm{i}) .{ }^{*} \mathrm{Wq}\right)+\operatorname{sum}\left(\mathrm{g} 1122 \mathrm{~b}(\mathrm{i}) \cdot{ }^{*} \mathrm{Wq}\right)+\operatorname{sum}\left(\mathrm{g} 2112 \mathrm{a}(\mathrm{i}) .{ }^{*} \mathrm{Wq}\right)+\operatorname{sum}(\mathrm{g} 21\right.\right.$ $12 \mathrm{~b}(\mathrm{i}) . * \mathrm{Wq}))$ ) $\left(\mathrm{L}(8,5) *\left(\operatorname{sum}(\mathrm{g} 1123 \mathrm{a}(\mathrm{i}) . * \mathrm{Wq})+\operatorname{sum}\left(\mathrm{g} 1123 \mathrm{~b}(\mathrm{i}) \cdot{ }^{*} \mathrm{Wq}\right)+\operatorname{sum}\left(\mathrm{g} 2113 \mathrm{a}(\mathrm{i}) .{ }^{*} \mathrm{Wq}\right)+\operatorname{sum}(\mathrm{g} 21\right.\right.$ $13 \mathrm{~b}(\mathrm{i}) . * \mathrm{Wq})))$ $\left(\mathrm{L}(9,5) *\left(\operatorname{sum}\left(\mathrm{g} 1221 \mathrm{a}(\mathrm{i}) .{ }^{*} \mathrm{Wq}\right)+\operatorname{sum}\left(\mathrm{g} 1221 \mathrm{~b}(\mathrm{i}) .{ }^{*} \mathrm{Wq}\right)+\operatorname{sum}\left(\mathrm{g} 2211 \mathrm{a}(\mathrm{i}) .{ }^{*} \mathrm{Wq}\right)+\operatorname{sum}(\mathrm{g} 22\right.\right.$ $11 \mathrm{~b}(\mathrm{i}) . * \mathrm{Wq})))$ $\left(\mathrm{L}(2,5) *\left(\operatorname{sum}\left(\mathrm{g} 1222 \mathrm{a}(\mathrm{i}) .{ }^{*} \mathrm{Wq}\right)+\operatorname{sum}\left(\mathrm{g} 1222 \mathrm{~b}(\mathrm{i}) \cdot{ }^{*} \mathrm{Wq}\right)+\operatorname{sum}\left(\mathrm{g} 2212 \mathrm{a}(\mathrm{i}) \cdot{ }^{*} \mathrm{Wq}\right)+\operatorname{sum}(\mathrm{g} 22\right.\right.$ $12 \mathrm{~b}(\mathrm{i}) . * \mathrm{Wq})))$ $\left(\mathrm{L}(4,5) *\left(\operatorname{sum}(\mathrm{g} 1223 \mathrm{a}(\mathrm{i}) . * \mathrm{Wq})+\operatorname{sum}\left(\mathrm{g} 1223 \mathrm{~b}(\mathrm{i}) \cdot{ }^{*} \mathrm{Wq}\right)+\operatorname{sum}\left(\mathrm{g} 2213 \mathrm{a}(\mathrm{i}) \cdot{ }^{*} \mathrm{Wq}\right)+\operatorname{sum}(\mathrm{g} 22\right.\right.$ $13 \mathrm{~b}(\mathrm{i}) . * \mathrm{Wq})))$ $\left(\mathrm{L}(5,5) *\left(\operatorname{sum}\left(\mathrm{g} 1321 \mathrm{a}(\mathrm{i}) .{ }^{*} \mathrm{Wq}\right)+\operatorname{sum}\left(\mathrm{g} 1321 \mathrm{~b}(\mathrm{i}) .{ }^{*} \mathrm{Wq}\right)+\operatorname{sum}\left(\mathrm{g} 2311 \mathrm{a}(\mathrm{i}) .{ }^{*} \mathrm{Wq}\right)+\operatorname{sum}(\mathrm{g} 23\right.\right.$ $11 \mathrm{~b}(\mathrm{i}) . * \mathrm{Wq})))$ $\left(\mathrm{L}(7,5) *\left(\operatorname{sum}\left(\mathrm{g} 1322 \mathrm{a}(\mathrm{i}) .{ }^{*} \mathrm{Wq}\right)+\operatorname{sum}\left(\mathrm{g} 1322 \mathrm{~b}(\mathrm{i}) .{ }^{*} \mathrm{Wq}\right)+\operatorname{sum}\left(\mathrm{g} 2312 \mathrm{a}(\mathrm{i}) .{ }^{*} \mathrm{Wq}\right)+\operatorname{sum}(\mathrm{g} 23\right.\right.$ $12 \mathrm{~b}(\mathrm{i}) . * \mathrm{Wq})))$ $\left(\mathrm{L}(3,5) *\left(\operatorname{sum}\left(\mathrm{g} 1323 \mathrm{a}(\mathrm{i}) .{ }^{*} \mathrm{Wq}\right)+\operatorname{sum}\left(\mathrm{g} 1323 \mathrm{~b}(\mathrm{i}) .{ }^{*} \mathrm{Wq}\right)+\operatorname{sum}\left(\mathrm{g} 2313 \mathrm{a}(\mathrm{i}) .{ }^{*} \mathrm{Wq}\right)+\operatorname{sum}(\mathrm{g} 23\right.\right.$ $13 \mathrm{~b}(\mathrm{i}) . * \mathrm{Wq})))) /(8 * \mathrm{pi})$;

$\mathrm{S} 1212$

$\left(\left(\mathrm{L}(1,6) *\left(\operatorname{sum}\left(\mathrm{g} 1121 \mathrm{a}(\mathrm{i}) .{ }^{*} \mathrm{Wq}\right)+\operatorname{sum}\left(\mathrm{g} 1121 \mathrm{~b}(\mathrm{i}) .{ }^{*} \mathrm{Wq}\right)+\operatorname{sum}\left(\mathrm{g} 2111 \mathrm{a}(\mathrm{i}) .{ }^{*} \mathrm{Wq}\right)+\operatorname{sum}(\mathrm{g} 2\right.\right.\right.$ $\left.\left.\left.111 \mathrm{~b}(\mathrm{i}) \cdot{ }^{*} \mathrm{Wq}\right)\right)\right)$ $\left(\mathrm{L}(6,6) *\left(\operatorname{sum}\left(\mathrm{g} 1122 \mathrm{a}(\mathrm{i}) .{ }^{*} \mathrm{Wq}\right)+\operatorname{sum}\left(\mathrm{g} 1122 \mathrm{~b}(\mathrm{i}) \cdot{ }^{*} \mathrm{Wq}\right)+\operatorname{sum}(\mathrm{g} 2112 \mathrm{a}(\mathrm{i}) . * \mathrm{Wq})+\operatorname{sum}(\mathrm{g} 21\right.\right.$ $12 \mathrm{~b}(\mathrm{i}) . * \mathrm{Wq})))$ $\left(\mathrm{L}(8,6) *\left(\operatorname{sum}(\mathrm{g} 1123 \mathrm{a}(\mathrm{i}) . * \mathrm{Wq})+\operatorname{sum}\left(\mathrm{g} 1123 \mathrm{~b}(\mathrm{i}) \cdot{ }^{*} \mathrm{Wq}\right)+\operatorname{sum}\left(\mathrm{g} 2113 \mathrm{a}(\mathrm{i}) \cdot{ }^{*} \mathrm{Wq}\right)+\operatorname{sum}(\mathrm{g} 21\right.\right.$ $13 \mathrm{~b}(\mathrm{i}) . * \mathrm{Wq})))$ $\left(\mathrm{L}(9,6) *\left(\operatorname{sum}\left(\mathrm{g} 1221 \mathrm{a}(\mathrm{i}) \cdot{ }^{*} \mathrm{Wq}\right)+\operatorname{sum}\left(\mathrm{g} 1221 \mathrm{~b}(\mathrm{i}) .{ }^{*} \mathrm{Wq}\right)+\operatorname{sum}\left(\mathrm{g} 2211 \mathrm{a}(\mathrm{i}) \cdot{ }^{*} \mathrm{Wq}\right)+\operatorname{sum}(\mathrm{g} 22\right.\right.$ $\left.\left.\left.11 \mathrm{~b}(\mathrm{i}) \cdot{ }^{*} \mathrm{Wq}\right)\right)\right)$ $\left(\mathrm{L}(2,6) *\left(\operatorname{sum}\left(\mathrm{g} 1222 \mathrm{a}(\mathrm{i}) .{ }^{*} \mathrm{Wq}\right)+\operatorname{sum}\left(\mathrm{g} 1222 \mathrm{~b}(\mathrm{i}) .{ }^{*} \mathrm{Wq}\right)+\operatorname{sum}\left(\mathrm{g} 2212 \mathrm{a}(\mathrm{i}) .{ }^{*} \mathrm{Wq}\right)+\operatorname{sum}(\mathrm{g} 22\right.\right.$ $12 \mathrm{~b}(\mathrm{i}) . * \mathrm{Wq})))$ $\left(\mathrm{L}(4,6) *\left(\operatorname{sum}(\mathrm{g} 1223 \mathrm{a}(\mathrm{i}) . * \mathrm{Wq})+\operatorname{sum}\left(\mathrm{g} 1223 \mathrm{~b}(\mathrm{i}) \cdot{ }^{*} \mathrm{Wq}\right)+\operatorname{sum}\left(\mathrm{g} 2213 \mathrm{a}(\mathrm{i}) \cdot{ }^{*} \mathrm{Wq}\right)+\operatorname{sum}(\mathrm{g} 22\right.\right.$ $13 \mathrm{~b}(\mathrm{i}) . * \mathrm{Wq})))$ $\left(\mathrm{L}(5,6) *\left(\operatorname{sum}\left(\mathrm{g} 1321 \mathrm{a}(\mathrm{i}) .{ }^{*} \mathrm{Wq}\right)+\operatorname{sum}\left(\mathrm{g} 1321 \mathrm{~b}(\mathrm{i}) .{ }^{*} \mathrm{Wq}\right)+\operatorname{sum}\left(\mathrm{g} 2311 \mathrm{a}(\mathrm{i}) .{ }^{*} \mathrm{Wq}\right)+\operatorname{sum}(\mathrm{g} 23\right.\right.$ 
$11 \mathrm{~b}(\mathrm{i}) . * \mathrm{Wq})))$

$\left(\mathrm{L}(7,6) *\left(\operatorname{sum}\left(\mathrm{g} 1322 \mathrm{a}(\mathrm{i}) .{ }^{*} \mathrm{Wq}\right)+\operatorname{sum}\left(\mathrm{g} 1322 \mathrm{~b}(\mathrm{i}) .{ }^{*} \mathrm{Wq}\right)+\operatorname{sum}\left(\mathrm{g} 2312 \mathrm{a}(\mathrm{i}) .{ }^{*} \mathrm{Wq}\right)+\operatorname{sum}(\mathrm{g} 23\right.\right.$

$12 \mathrm{~b}(\mathrm{i}) . * \mathrm{Wq}))$ )

$\left(\mathrm{L}(3,6) *\left(\operatorname{sum}\left(\mathrm{g} 1323 \mathrm{a}(\mathrm{i}) .{ }^{*} \mathrm{Wq}\right)+\operatorname{sum}\left(\mathrm{g} 1323 \mathrm{~b}(\mathrm{i}) .{ }^{*} \mathrm{Wq}\right)+\operatorname{sum}\left(\mathrm{g} 2313 \mathrm{a}(\mathrm{i}) .{ }^{*} \mathrm{Wq}\right)+\operatorname{sum}(\mathrm{g} 23\right.\right.$

$\left.\left.\left.\left.13 \mathrm{~b}(\mathrm{i}) \cdot{ }^{*} \mathrm{Wq}\right)\right)\right)\right) /(8 * \mathrm{pi})$;

$\mathrm{S} 1232$

$\left(\left(\mathrm{L}(1,7) *\left(\operatorname{sum}\left(\mathrm{g} 1121 \mathrm{a}(\mathrm{i}) .{ }^{*} \mathrm{Wq}\right)+\operatorname{sum}\left(\mathrm{g} 1121 \mathrm{~b}(\mathrm{i}) .{ }^{*} \mathrm{Wq}\right)+\operatorname{sum}\left(\mathrm{g} 2111 \mathrm{a}(\mathrm{i}) .{ }^{*} \mathrm{Wq}\right)+\operatorname{sum}(\mathrm{g} 2\right.\right.\right.$ $\left.\left.\left.111 \mathrm{~b}(\mathrm{i}) *{ }^{*} \mathrm{Wq}\right)\right)\right)$

$\left(\mathrm{L}(6,7) *\left(\operatorname{sum}\left(\mathrm{g} 1122 \mathrm{a}(\mathrm{i}) .{ }^{*} \mathrm{Wq}\right)+\operatorname{sum}\left(\mathrm{g} 1122 \mathrm{~b}(\mathrm{i}) .{ }^{*} \mathrm{Wq}\right)+\operatorname{sum}\left(\mathrm{g} 2112 \mathrm{a}(\mathrm{i}) .{ }^{*} \mathrm{Wq}\right)+\operatorname{sum}(\mathrm{g} 21\right.\right.$

$12 \mathrm{~b}(\mathrm{i}) . * \mathrm{Wq})))$

$\left(\mathrm{L}(8,7) *\left(\operatorname{sum}\left(\mathrm{g} 1123 \mathrm{a}(\mathrm{i}) .{ }^{*} \mathrm{Wq}\right)+\operatorname{sum}\left(\mathrm{g} 1123 \mathrm{~b}(\mathrm{i}) .{ }^{*} \mathrm{Wq}\right)+\operatorname{sum}(\mathrm{g} 2113 \mathrm{a}(\mathrm{i}) . * \mathrm{Wq})+\operatorname{sum}(\mathrm{g} 21\right.\right.$

$13 \mathrm{~b}(\mathrm{i}) . \mathrm{Wq})))$

$\left(\mathrm{L}(9,7) *\left(\operatorname{sum}\left(\mathrm{g} 1221 \mathrm{a}(\mathrm{i}) \cdot{ }^{*} \mathrm{Wq}\right)+\operatorname{sum}\left(\mathrm{g} 1221 \mathrm{~b}(\mathrm{i}) \cdot{ }^{*} \mathrm{Wq}\right)+\operatorname{sum}\left(\mathrm{g} 2211 \mathrm{a}(\mathrm{i}) \cdot{ }^{*} \mathrm{Wq}\right)+\operatorname{sum}(\mathrm{g} 22\right.\right.$

$11 \mathrm{~b}(\mathrm{i}) . * \mathrm{Wq}))$

$\left(\mathrm{L}(2,7) *\left(\operatorname{sum}\left(\mathrm{g} 1222 \mathrm{a}(\mathrm{i}) .{ }^{*} \mathrm{Wq}\right)+\operatorname{sum}\left(\mathrm{g} 1222 \mathrm{~b}(\mathrm{i}) .{ }^{*} \mathrm{Wq}\right)+\operatorname{sum}\left(\mathrm{g} 2212 \mathrm{a}(\mathrm{i}) .{ }^{*} \mathrm{Wq}\right)+\operatorname{sum}(\mathrm{g} 22\right.\right.$

$12 \mathrm{~b}(\mathrm{i}) . * \mathrm{Wq})))$

$\left(\mathrm{L}(4,7) *\left(\operatorname{sum}\left(\mathrm{g} 1223 \mathrm{a}(\mathrm{i}) .{ }^{*} \mathrm{Wq}\right)+\operatorname{sum}(\mathrm{g} 1223 \mathrm{~b}(\mathrm{i}) . * \mathrm{Wq})+\operatorname{sum}(\mathrm{g} 2213 \mathrm{a}(\mathrm{i}) . * \mathrm{Wq})+\operatorname{sum}(\mathrm{g} 22\right.\right.$

$\left.\left.\left.13 \mathrm{~b}(\mathrm{i}) \cdot{ }^{*} \mathrm{Wq}\right)\right)\right)$

$\left(\mathrm{L}(5,7) *\left(\operatorname{sum}\left(\mathrm{g} 1321 \mathrm{a}(\mathrm{i}) .{ }^{*} \mathrm{Wq}\right)+\operatorname{sum}\left(\mathrm{g} 1321 \mathrm{~b}(\mathrm{i}) .{ }^{*} \mathrm{Wq}\right)+\operatorname{sum}\left(\mathrm{g} 2311 \mathrm{a}(\mathrm{i}) .{ }^{*} \mathrm{Wq}\right)+\operatorname{sum}(\mathrm{g} 23\right.\right.$

$11 \mathrm{~b}(\mathrm{i}) . * \mathrm{Wq})))$

$\left(\mathrm{L}(7,7) *\left(\operatorname{sum}\left(\mathrm{g} 1322 \mathrm{a}(\mathrm{i}) .{ }^{*} \mathrm{Wq}\right)+\operatorname{sum}\left(\mathrm{g} 1322 \mathrm{~b}(\mathrm{i}) \cdot{ }^{*} \mathrm{Wq}\right)+\operatorname{sum}\left(\mathrm{g} 2312 \mathrm{a}(\mathrm{i}) .{ }^{*} \mathrm{Wq}\right)+\operatorname{sum}(\mathrm{g} 23\right.\right.$

$12 \mathrm{~b}(\mathrm{i}) . * \mathrm{Wq})))$

$\left(\mathrm{L}(3,7) *\left(\operatorname{sum}\left(\mathrm{g} 1323 \mathrm{a}(\mathrm{i}) .{ }^{*} \mathrm{Wq}\right)+\operatorname{sum}\left(\mathrm{g} 1323 \mathrm{~b}(\mathrm{i}) .{ }^{*} \mathrm{Wq}\right)+\operatorname{sum}\left(\mathrm{g} 2313 \mathrm{a}(\mathrm{i}) .{ }^{*} \mathrm{Wq}\right)+\operatorname{sum}(\mathrm{g} 23\right.\right.$

$\left.\left.\left.\left.13 \mathrm{~b}(\mathrm{i}) *{ }^{*} \mathrm{Wq}\right)\right)\right)\right) /(8 * \mathrm{pi})$;

$\mathrm{S} 1213$

$\left(\left(\mathrm{L}(1,8) *\left(\operatorname{sum}\left(\mathrm{g} 1121 \mathrm{a}(\mathrm{i}) .{ }^{*} \mathrm{Wq}\right)+\operatorname{sum}\left(\mathrm{g} 1121 \mathrm{~b}(\mathrm{i}) .{ }^{*} \mathrm{Wq}\right)+\operatorname{sum}\left(\mathrm{g} 2111 \mathrm{a}(\mathrm{i}) .{ }^{*} \mathrm{Wq}\right)+\operatorname{sum}(\mathrm{g} 2\right.\right.\right.$ $111 \mathrm{~b}(\mathrm{i}) \cdot * \mathrm{Wq})))$

$\left(\mathrm{L}(6,8) *\left(\operatorname{sum}\left(\mathrm{g} 1122 \mathrm{a}(\mathrm{i}) .{ }^{*} \mathrm{Wq}\right)+\operatorname{sum}\left(\mathrm{g} 1122 \mathrm{~b}(\mathrm{i}) .{ }^{*} \mathrm{Wq}\right)+\operatorname{sum}\left(\mathrm{g} 2112 \mathrm{a}(\mathrm{i}) .{ }^{*} \mathrm{Wq}\right)+\operatorname{sum}(\mathrm{g} 21\right.\right.$

$12 \mathrm{~b}(\mathrm{i}) . * \mathrm{Wq}))$ )

$\left(\mathrm{L}(8,8) *\left(\operatorname{sum}\left(\mathrm{g} 1123 \mathrm{a}(\mathrm{i}) .{ }^{*} \mathrm{Wq}\right)+\operatorname{sum}\left(\mathrm{g} 1123 \mathrm{~b}(\mathrm{i}) .{ }^{*} \mathrm{Wq}\right)+\operatorname{sum}\left(\mathrm{g} 2113 \mathrm{a}(\mathrm{i}) \cdot{ }^{*} \mathrm{Wq}\right)+\operatorname{sum}(\mathrm{g} 21\right.\right.$

$13 \mathrm{~b}(\mathrm{i}) . * \mathrm{Wq})))$

$\left(\mathrm{L}(9,8) *\left(\operatorname{sum}\left(\mathrm{g} 1221 \mathrm{a}(\mathrm{i}) .{ }^{*} \mathrm{Wq}\right)+\operatorname{sum}\left(\mathrm{g} 1221 \mathrm{~b}(\mathrm{i}) .{ }^{*} \mathrm{Wq}\right)+\operatorname{sum}\left(\mathrm{g} 2211 \mathrm{a}(\mathrm{i}) .{ }^{*} \mathrm{Wq}\right)+\operatorname{sum}(\mathrm{g} 22\right.\right.$

$11 \mathrm{~b}(\mathrm{i}) . * \mathrm{Wq})))$

$\left(\mathrm{L}(2,8) *\left(\operatorname{sum}\left(\mathrm{g} 1222 \mathrm{a}(\mathrm{i}) .{ }^{*} \mathrm{Wq}\right)+\operatorname{sum}\left(\mathrm{g} 1222 \mathrm{~b}(\mathrm{i}) \cdot{ }^{*} \mathrm{Wq}\right)+\operatorname{sum}\left(\mathrm{g} 2212 \mathrm{a}(\mathrm{i}) .{ }^{*} \mathrm{Wq}\right)+\operatorname{sum}(\mathrm{g} 22\right.\right.$

$12 \mathrm{~b}(\mathrm{i}) . * \mathrm{Wq})))$

$\left(\mathrm{L}(4,8) *\left(\operatorname{sum}\left(\mathrm{g} 1223 \mathrm{a}(\mathrm{i}) .{ }^{*} \mathrm{Wq}\right)+\operatorname{sum}\left(\mathrm{g} 1223 \mathrm{~b}(\mathrm{i}) \cdot{ }^{*} \mathrm{Wq}\right)+\operatorname{sum}\left(\mathrm{g} 2213 \mathrm{a}(\mathrm{i}) .{ }^{*} \mathrm{Wq}\right)+\operatorname{sum}(\mathrm{g} 22\right.\right.$

$\left.\left.\left.13 \mathrm{~b}(\mathrm{i}) \cdot{ }^{*} \mathrm{Wq}\right)\right)\right)$

$\left(\mathrm{L}(5,8) *\left(\operatorname{sum}\left(\mathrm{g} 1321 \mathrm{a}(\mathrm{i}) .{ }^{*} \mathrm{Wq}\right)+\operatorname{sum}\left(\mathrm{g} 1321 \mathrm{~b}(\mathrm{i}) .{ }^{*} \mathrm{Wq}\right)+\operatorname{sum}\left(\mathrm{g} 2311 \mathrm{a}(\mathrm{i}) .{ }^{*} \mathrm{Wq}\right)+\operatorname{sum}(\mathrm{g} 23\right.\right.$

$11 \mathrm{~b}(\mathrm{i}) . * \mathrm{Wq})))$

$\left(\mathrm{L}(7,8) *\left(\operatorname{sum}\left(\mathrm{g} 1322 \mathrm{a}(\mathrm{i}) .{ }^{*} \mathrm{Wq}\right)+\operatorname{sum}\left(\mathrm{g} 1322 \mathrm{~b}(\mathrm{i}) \cdot{ }^{*} \mathrm{Wq}\right)+\operatorname{sum}\left(\mathrm{g} 2312 \mathrm{a}(\mathrm{i}) \cdot{ }^{*} \mathrm{Wq}\right)+\operatorname{sum}(\mathrm{g} 23\right.\right.$

$12 \mathrm{~b}(\mathrm{i}) . * \mathrm{Wq})))$

$\left(\mathrm{L}(3,8) *\left(\operatorname{sum}\left(\mathrm{g} 1323 \mathrm{a}(\mathrm{i}) .{ }^{*} \mathrm{Wq}\right)+\operatorname{sum}\left(\mathrm{g} 1323 \mathrm{~b}(\mathrm{i}) \cdot{ }^{*} \mathrm{Wq}\right)+\operatorname{sum}\left(\mathrm{g} 2313 \mathrm{a}(\mathrm{i}) \cdot{ }^{*} \mathrm{Wq}\right)+\operatorname{sum}(\mathrm{g} 23\right.\right.$

$\left.\left.\left.\left.13 \mathrm{~b}(\mathrm{i}){ }^{*} \mathrm{Wq}\right)\right)\right)\right) /(8 * \mathrm{pi})$; 
S1221

$\left(\left(\mathrm{L}(1,9) *\left(\operatorname{sum}\left(\mathrm{g} 1121 \mathrm{a}(\mathrm{i}) .{ }^{*} \mathrm{Wq}\right)+\operatorname{sum}\left(\mathrm{g} 1121 \mathrm{~b}(\mathrm{i}) .{ }^{*} \mathrm{Wq}\right)+\operatorname{sum}\left(\mathrm{g} 2111 \mathrm{a}(\mathrm{i}) .{ }^{*} \mathrm{Wq}\right)+\operatorname{sum}(\mathrm{g} 2\right.\right.\right.$ $111 b(\mathrm{i}) \cdot * \mathrm{Wq})))$

$\left(\mathrm{L}(6,9) *\left(\operatorname{sum}\left(\mathrm{g} 1122 \mathrm{a}(\mathrm{i}) .{ }^{*} \mathrm{Wq}\right)+\operatorname{sum}\left(\mathrm{g} 1122 \mathrm{~b}(\mathrm{i}) \cdot{ }^{*} \mathrm{Wq}\right)+\operatorname{sum}\left(\mathrm{g} 2112 \mathrm{a}(\mathrm{i}) .{ }^{*} \mathrm{Wq}\right)+\operatorname{sum}(\mathrm{g} 21\right.\right.$ $12 \mathrm{~b}(\mathrm{i}) . * \mathrm{Wq})))$

$\left(\mathrm{L}(8,9) *\left(\operatorname{sum}\left(\mathrm{g} 1123 \mathrm{a}(\mathrm{i}) .{ }^{*} \mathrm{Wq}\right)+\operatorname{sum}\left(\mathrm{g} 1123 \mathrm{~b}(\mathrm{i}) .{ }^{*} \mathrm{Wq}\right)+\operatorname{sum}\left(\mathrm{g} 2113 \mathrm{a}(\mathrm{i}) .{ }^{*} \mathrm{Wq}\right)+\operatorname{sum}(\mathrm{g} 21\right.\right.$ $13 \mathrm{~b}(\mathrm{i}) . * \mathrm{Wq})))$

$\left(\mathrm{L}(9,9) *\left(\operatorname{sum}\left(\mathrm{g} 1221 \mathrm{a}(\mathrm{i}) .{ }^{*} \mathrm{Wq}\right)+\operatorname{sum}\left(\mathrm{g} 1221 \mathrm{~b}(\mathrm{i}) \cdot{ }^{*} \mathrm{Wq}\right)+\operatorname{sum}\left(\mathrm{g} 2211 \mathrm{a}(\mathrm{i}) .{ }^{*} \mathrm{Wq}\right)+\operatorname{sum}(\mathrm{g} 22\right.\right.$ $11 \mathrm{~b}(\mathrm{i}) . * \mathrm{Wq})))$

$\left(\mathrm{L}(2,9) *\left(\operatorname{sum}\left(\mathrm{g} 1222 \mathrm{a}(\mathrm{i}) \cdot{ }^{*} \mathrm{Wq}\right)+\operatorname{sum}\left(\mathrm{g} 1222 \mathrm{~b}(\mathrm{i}) .{ }^{*} \mathrm{Wq}\right)+\operatorname{sum}\left(\mathrm{g} 2212 \mathrm{a}(\mathrm{i}) .{ }^{*} \mathrm{Wq}\right)+\operatorname{sum}(\mathrm{g} 22\right.\right.$ $12 \mathrm{~b}(\mathrm{i}) . * \mathrm{Wq})))$

$\left(\mathrm{L}(4,9) *\left(\operatorname{sum}\left(\mathrm{g} 1223 \mathrm{a}(\mathrm{i}) .{ }^{*} \mathrm{Wq}\right)+\operatorname{sum}\left(\mathrm{g} 1223 \mathrm{~b}(\mathrm{i}) .{ }^{*} \mathrm{Wq}\right)+\operatorname{sum}\left(\mathrm{g} 2213 \mathrm{a}(\mathrm{i}) .{ }^{*} \mathrm{Wq}\right)+\operatorname{sum}(\mathrm{g} 22\right.\right.$ $13 \mathrm{~b}(\mathrm{i}) . * \mathrm{Wq})))$

$\left(\mathrm{L}(5,9) *\left(\operatorname{sum}\left(\mathrm{g} 1321 \mathrm{a}(\mathrm{i}) .{ }^{*} \mathrm{Wq}\right)+\operatorname{sum}\left(\mathrm{g} 1321 \mathrm{~b}(\mathrm{i}) \cdot{ }^{*} \mathrm{Wq}\right)+\operatorname{sum}\left(\mathrm{g} 2311 \mathrm{a}(\mathrm{i}) .{ }^{*} \mathrm{Wq}\right)+\operatorname{sum}(\mathrm{g} 23\right.\right.$ $11 \mathrm{~b}(\mathrm{i}) . * \mathrm{Wq})))$

$\left(\mathrm{L}(7,9) *\left(\operatorname{sum}\left(\mathrm{g} 1322 \mathrm{a}(\mathrm{i}) .{ }^{*} \mathrm{Wq}\right)+\operatorname{sum}\left(\mathrm{g} 1322 \mathrm{~b}(\mathrm{i}) \cdot{ }^{*} \mathrm{Wq}\right)+\operatorname{sum}\left(\mathrm{g} 2312 \mathrm{a}(\mathrm{i}) .{ }^{*} \mathrm{Wq}\right)+\operatorname{sum}(\mathrm{g} 23\right.\right.$ $12 \mathrm{~b}(\mathrm{i}) . * \mathrm{Wq}))$ )

$\left(\mathrm{L}(3,9) *\left(\operatorname{sum}\left(\mathrm{g} 1323 \mathrm{a}(\mathrm{i}) \cdot{ }^{*} \mathrm{Wq}\right)+\operatorname{sum}\left(\mathrm{g} 1323 \mathrm{~b}(\mathrm{i}) \cdot{ }^{*} \mathrm{Wq}\right)+\operatorname{sum}\left(\mathrm{g} 2313 \mathrm{a}(\mathrm{i}) \cdot{ }^{*} \mathrm{Wq}\right)+\operatorname{sum}(\mathrm{g} 23\right.\right.$ $\left.\left.\left.\left.13 \mathrm{~b}(\mathrm{i}) *{ }^{*} \mathrm{Wq}\right)\right)\right)\right) /(8 * \mathrm{pi})$;

S3211

$\left(\left(\mathrm{L}(1,1) *\left(\operatorname{sum}(\mathrm{g} 3121 \mathrm{a}(\mathrm{i}) . * \mathrm{Wq})+\operatorname{sum}\left(\mathrm{g} 3121 \mathrm{~b}(\mathrm{i}) .{ }^{*} \mathrm{Wq}\right)+\operatorname{sum}(\mathrm{g} 2131 \mathrm{a}(\mathrm{i}) . * \mathrm{Wq})+\operatorname{sum}(\mathrm{g} 2\right.\right.\right.$ $\left.\left.\left.131 \mathrm{~b}(\mathrm{i}) *{ }^{*} \mathrm{Wq}\right)\right)\right)$

$\left(\mathrm{L}(6,1) *\left(\operatorname{sum}\left(\mathrm{g} 3122 \mathrm{a}(\mathrm{i}) .{ }^{*} \mathrm{Wq}\right)+\operatorname{sum}(\mathrm{g} 3122 \mathrm{~b}(\mathrm{i}) . * \mathrm{Wq})+\operatorname{sum}(\mathrm{g} 2132 \mathrm{a}(\mathrm{i}) . * \mathrm{Wq})+\operatorname{sum}(\mathrm{g} 21\right.\right.$ $32 \mathrm{~b}(\mathrm{i}) . * \mathrm{Wq})))$

$\left(\mathrm{L}(8,1) *\left(\operatorname{sum}\left(\mathrm{g} 3123 \mathrm{a}(\mathrm{i}) .{ }^{*} \mathrm{Wq}\right)+\operatorname{sum}\left(\mathrm{g} 3123 \mathrm{~b}(\mathrm{i}) \cdot{ }^{*} \mathrm{Wq}\right)+\operatorname{sum}\left(\mathrm{g} 2133 \mathrm{a}(\mathrm{i}) \cdot{ }^{*} \mathrm{Wq}\right)+\operatorname{sum}(\mathrm{g} 21\right.\right.$ $33 \mathrm{~b}(\mathrm{i}) . * \mathrm{Wq}))$ )

$\left(\mathrm{L}(9,1) *\left(\operatorname{sum}\left(\mathrm{g} 3221 \mathrm{a}(\mathrm{i}) .{ }^{*} \mathrm{Wq}\right)+\operatorname{sum}\left(\mathrm{g} 3221 \mathrm{~b}(\mathrm{i}) .{ }^{*} \mathrm{Wq}\right)+\operatorname{sum}\left(\mathrm{g} 2231 \mathrm{a}(\mathrm{i}) .{ }^{*} \mathrm{Wq}\right)+\operatorname{sum}(\mathrm{g} 22\right.\right.$ $\left.\left.\left.31 \mathrm{~b}(\mathrm{i}) *{ }^{*} \mathrm{Wq}\right)\right)\right)$

$\left(\mathrm{L}(2,1) *\left(\operatorname{sum}\left(\mathrm{g} 3222 \mathrm{a}(\mathrm{i}) .{ }^{*} \mathrm{Wq}\right)+\operatorname{sum}\left(\mathrm{g} 3222 \mathrm{~b}(\mathrm{i}) .{ }^{*} \mathrm{Wq}\right)+\operatorname{sum}\left(\mathrm{g} 2232 \mathrm{a}(\mathrm{i}) .{ }^{*} \mathrm{Wq}\right)+\operatorname{sum}(\mathrm{g} 22\right.\right.$ $32 \mathrm{~b}(\mathrm{i}) . * \mathrm{Wq}))$ )

$\left(\mathrm{L}(4,1) *\left(\operatorname{sum}\left(\mathrm{g} 3223 \mathrm{a}(\mathrm{i}) .{ }^{*} \mathrm{Wq}\right)+\operatorname{sum}\left(\mathrm{g} 3223 \mathrm{~b}(\mathrm{i}) \cdot{ }^{*} \mathrm{Wq}\right)+\operatorname{sum}\left(\mathrm{g} 2233 \mathrm{a}(\mathrm{i}) .{ }^{*} \mathrm{Wq}\right)+\operatorname{sum}(\mathrm{g} 22\right.\right.$ $33 \mathrm{~b}(\mathrm{i}) . * \mathrm{Wq}))$ )

$\left(\mathrm{L}(5,1) *\left(\operatorname{sum}\left(\mathrm{g} 3321 \mathrm{a}(\mathrm{i}) .{ }^{*} \mathrm{Wq}\right)+\operatorname{sum}\left(\mathrm{g} 3321 \mathrm{~b}(\mathrm{i}) .{ }^{*} \mathrm{Wq}\right)+\operatorname{sum}\left(\mathrm{g} 2331 \mathrm{a}(\mathrm{i}) .{ }^{*} \mathrm{Wq}\right)+\operatorname{sum}(\mathrm{g} 23\right.\right.$ $31 \mathrm{~b}(\mathrm{i}) . * \mathrm{Wq})))$

$\left(\mathrm{L}(7,1) *\left(\operatorname{sum}(\mathrm{g} 3322 \mathrm{a}(\mathrm{i}) . * \mathrm{Wq})+\operatorname{sum}\left(\mathrm{g} 3322 \mathrm{~b}(\mathrm{i}) \cdot{ }^{*} \mathrm{Wq}\right)+\operatorname{sum}\left(\mathrm{g} 2332 \mathrm{a}(\mathrm{i}) \cdot{ }^{*} \mathrm{Wq}\right)+\operatorname{sum}(\mathrm{g} 23\right.\right.$ $32 \mathrm{~b}(\mathrm{i}) . * \mathrm{Wq}))$ )

$\left(\mathrm{L}(3,1) *\left(\operatorname{sum}\left(\mathrm{g} 3323 \mathrm{a}(\mathrm{i}) \cdot{ }^{*} \mathrm{Wq}\right)+\operatorname{sum}\left(\mathrm{g} 3323 \mathrm{~b}(\mathrm{i}) \cdot{ }^{*} \mathrm{Wq}\right)+\operatorname{sum}\left(\mathrm{g} 2333 \mathrm{a}(\mathrm{i}) \cdot{ }^{*} \mathrm{Wq}\right)+\operatorname{sum}(\mathrm{g} 23\right.\right.$ $33 \mathrm{~b}(\mathrm{i}) *$ (Wq) $))) /(8 * \mathrm{pi})$;

S 3222

$\left(\left(\mathrm{L}(1,2) *\left(\operatorname{sum}\left(\mathrm{g} 3121 \mathrm{a}(\mathrm{i}) .{ }^{*} \mathrm{Wq}\right)+\operatorname{sum}\left(\mathrm{g} 3121 \mathrm{~b}(\mathrm{i}){ }^{*} \mathrm{Wq}\right)+\operatorname{sum}\left(\mathrm{g} 2131 \mathrm{a}(\mathrm{i}) .{ }^{*} \mathrm{Wq}\right)+\operatorname{sum}(\mathrm{g} 2\right.\right.\right.$ $131 \mathrm{~b}(\mathrm{i}) . * \mathrm{Wq})))$

$\left(\mathrm{L}(6,2) *\left(\operatorname{sum}\left(\mathrm{g} 3122 \mathrm{a}(\mathrm{i}) .{ }^{*} \mathrm{Wq}\right)+\operatorname{sum}\left(\mathrm{g} 3122 \mathrm{~b}(\mathrm{i}) .{ }^{*} \mathrm{Wq}\right)+\operatorname{sum}\left(\mathrm{g} 2132 \mathrm{a}(\mathrm{i}) .{ }^{*} \mathrm{Wq}\right)+\operatorname{sum}(\mathrm{g} 21\right.\right.$ 
$32 \mathrm{~b}(\mathrm{i}) \cdot * \mathrm{Wq})))$

$\left(\mathrm{L}(8,2) *\left(\operatorname{sum}\left(\mathrm{g} 3123 \mathrm{a}(\mathrm{i}) .{ }^{*} \mathrm{Wq}\right)+\operatorname{sum}\left(\mathrm{g} 3123 \mathrm{~b}(\mathrm{i}) \cdot{ }^{*} \mathrm{Wq}\right)+\operatorname{sum}\left(\mathrm{g} 2133 \mathrm{a}(\mathrm{i}) .{ }^{*} \mathrm{Wq}\right)+\operatorname{sum}(\mathrm{g} 21\right.\right.$

$33 \mathrm{~b}(\mathrm{i}) . * \mathrm{Wq}))$ )

$\left(\mathrm{L}(9,2) *\left(\operatorname{sum}\left(\mathrm{g} 3221 \mathrm{a}(\mathrm{i}) .{ }^{*} \mathrm{Wq}\right)+\operatorname{sum}\left(\mathrm{g} 3221 \mathrm{~b}(\mathrm{i}) \cdot{ }^{*} \mathrm{Wq}\right)+\operatorname{sum}\left(\mathrm{g} 2231 \mathrm{a}(\mathrm{i}) \cdot{ }^{*} \mathrm{Wq}\right)+\operatorname{sum}(\mathrm{g} 22\right.\right.$

$31 \mathrm{~b}(\mathrm{i}) . * \mathrm{Wq}))$

$\left(\mathrm{L}(2,2) *\left(\operatorname{sum}\left(\mathrm{g} 3222 \mathrm{a}(\mathrm{i}) .{ }^{*} \mathrm{Wq}\right)+\operatorname{sum}\left(\mathrm{g} 3222 \mathrm{~b}(\mathrm{i}) \cdot{ }^{*} \mathrm{Wq}\right)+\operatorname{sum}\left(\mathrm{g} 2232 \mathrm{a}(\mathrm{i}) \cdot{ }^{*} \mathrm{Wq}\right)+\operatorname{sum}(\mathrm{g} 22\right.\right.$

$32 \mathrm{~b}(\mathrm{i}) . * \mathrm{Wq})))$

$\left(\mathrm{L}(4,2) *\left(\operatorname{sum}\left(\mathrm{g} 3223 \mathrm{a}(\mathrm{i}) .{ }^{*} \mathrm{Wq}\right)+\operatorname{sum}\left(\mathrm{g} 3223 \mathrm{~b}(\mathrm{i}) .{ }^{*} \mathrm{Wq}\right)+\operatorname{sum}\left(\mathrm{g} 2233 \mathrm{a}(\mathrm{i}) .{ }^{*} \mathrm{Wq}\right)+\operatorname{sum}(\mathrm{g} 22\right.\right.$

$33 b(\mathrm{i}) \cdot * \mathrm{Wq}))$

$\left(\mathrm{L}(5,2) *\left(\operatorname{sum}\left(\mathrm{g} 3321 \mathrm{a}(\mathrm{i}) .{ }^{*} \mathrm{Wq}\right)+\operatorname{sum}\left(\mathrm{g} 3321 \mathrm{~b}(\mathrm{i}) .{ }^{*} \mathrm{Wq}\right)+\operatorname{sum}\left(\mathrm{g} 2331 \mathrm{a}(\mathrm{i}) .{ }^{*} \mathrm{Wq}\right)+\operatorname{sum}(\mathrm{g} 23\right.\right.$

$31 \mathrm{~b}(\mathrm{i}) . * \mathrm{Wq})))$

$\left(\mathrm{L}(7,2) *\left(\operatorname{sum}\left(\mathrm{g} 3322 \mathrm{a}(\mathrm{i}) .{ }^{*} \mathrm{Wq}\right)+\operatorname{sum}\left(\mathrm{g} 3322 \mathrm{~b}(\mathrm{i}) .{ }^{*} \mathrm{Wq}\right)+\operatorname{sum}\left(\mathrm{g} 2332 \mathrm{a}(\mathrm{i}) .{ }^{*} \mathrm{Wq}\right)+\operatorname{sum}(\mathrm{g} 23\right.\right.$

$32 \mathrm{~b}(\mathrm{i}) . * \mathrm{Wq}))$

$\left(\mathrm{L}(3,2) *\left(\operatorname{sum}\left(\mathrm{g} 3323 \mathrm{a}(\mathrm{i}) .{ }^{*} \mathrm{Wq}\right)+\operatorname{sum}\left(\mathrm{g} 3323 \mathrm{~b}(\mathrm{i}) \cdot{ }^{*} \mathrm{Wq}\right)+\operatorname{sum}\left(\mathrm{g} 2333 \mathrm{a}(\mathrm{i}) .{ }^{*} \mathrm{Wq}\right)+\operatorname{sum}(\mathrm{g} 23\right.\right.$

$\left.\left.\left.\left.33 \mathrm{~b}(\mathrm{i}){ }^{*} \mathrm{Wq}\right)\right)\right)\right) /(8 * \mathrm{pi})$;

S3233

$((\mathrm{L}(1,3) *(\operatorname{sum}(\mathrm{g} 3121 \mathrm{a}(\mathrm{i}) . * \mathrm{Wq})+\operatorname{sum}(\mathrm{g} 3121 \mathrm{~b}(\mathrm{i}) . * \mathrm{Wq})+\operatorname{sum}(\mathrm{g} 2131 \mathrm{a}(\mathrm{i}) . * \mathrm{Wq})+\operatorname{sum}(\mathrm{g} 2$ $\left.\left.\left.131 \mathrm{~b}(\mathrm{i}) \cdot{ }^{*} \mathrm{Wq}\right)\right)\right)$

$\left(\mathrm{L}(6,3) *\left(\operatorname{sum}\left(\mathrm{g} 3122 \mathrm{a}(\mathrm{i}) .{ }^{*} \mathrm{Wq}\right)+\operatorname{sum}\left(\mathrm{g} 3122 \mathrm{~b}(\mathrm{i}) .{ }^{*} \mathrm{Wq}\right)+\operatorname{sum}(\mathrm{g} 2132 \mathrm{a}(\mathrm{i}) . * \mathrm{Wq})+\operatorname{sum}(\mathrm{g} 21\right.\right.$ $32 \mathrm{~b}(\mathrm{i}) . * \mathrm{Wq})))$

$\left(\mathrm{L}(8,3) *\left(\operatorname{sum}\left(\mathrm{g} 3123 \mathrm{a}(\mathrm{i}) .{ }^{*} \mathrm{Wq}\right)+\operatorname{sum}\left(\mathrm{g} 3123 \mathrm{~b}(\mathrm{i}) \cdot{ }^{*} \mathrm{Wq}\right)+\operatorname{sum}\left(\mathrm{g} 2133 \mathrm{a}(\mathrm{i}) .{ }^{*} \mathrm{Wq}\right)+\operatorname{sum}(\mathrm{g} 21\right.\right.$

$33 \mathrm{~b}(\mathrm{i}) . * \mathrm{Wq}))$

$\left(\mathrm{L}(9,3) *\left(\operatorname{sum}\left(\mathrm{g} 3221 \mathrm{a}(\mathrm{i}) .{ }^{*} \mathrm{Wq}\right)+\operatorname{sum}\left(\mathrm{g} 3221 \mathrm{~b}(\mathrm{i}) .{ }^{*} \mathrm{Wq}\right)+\operatorname{sum}\left(\mathrm{g} 2231 \mathrm{a}(\mathrm{i}) .{ }^{*} \mathrm{Wq}\right)+\operatorname{sum}(\mathrm{g} 22\right.\right.$

$31 \mathrm{~b}(\mathrm{i}) \cdot * \mathrm{Wq}))$

$\left(\mathrm{L}(2,3) *\left(\operatorname{sum}\left(\mathrm{g} 3222 \mathrm{a}(\mathrm{i}) .{ }^{*} \mathrm{Wq}\right)+\operatorname{sum}\left(\mathrm{g} 3222 \mathrm{~b}(\mathrm{i}) .{ }^{*} \mathrm{Wq}\right)+\operatorname{sum}\left(\mathrm{g} 2232 \mathrm{a}(\mathrm{i}) \cdot{ }^{*} \mathrm{Wq}\right)+\operatorname{sum}(\mathrm{g} 22\right.\right.$

$\left.\left.\left.32 \mathrm{~b}(\mathrm{i}) \cdot{ }^{*} \mathrm{Wq}\right)\right)\right)$

$\left(\mathrm{L}(4,3) *\left(\operatorname{sum}\left(\mathrm{g} 3223 \mathrm{a}(\mathrm{i}) .{ }^{*} \mathrm{Wq}\right)+\operatorname{sum}\left(\mathrm{g} 3223 \mathrm{~b}(\mathrm{i}) .{ }^{*} \mathrm{Wq}\right)+\operatorname{sum}\left(\mathrm{g} 2233 \mathrm{a}(\mathrm{i}) .{ }^{*} \mathrm{Wq}\right)+\operatorname{sum}(\mathrm{g} 22\right.\right.$

$\left.\left.33 \mathrm{~b}(\mathrm{i}) \cdot{ }^{*} \mathrm{Wq}\right)\right)$ )

$\left(\mathrm{L}(5,3) *\left(\operatorname{sum}\left(\mathrm{g} 3321 \mathrm{a}(\mathrm{i}) .{ }^{*} \mathrm{Wq}\right)+\operatorname{sum}\left(\mathrm{g} 3321 \mathrm{~b}(\mathrm{i}) \cdot{ }^{*} \mathrm{Wq}\right)+\operatorname{sum}\left(\mathrm{g} 2331 \mathrm{a}(\mathrm{i}) .{ }^{*} \mathrm{Wq}\right)+\operatorname{sum}(\mathrm{g} 23\right.\right.$

$31 \mathrm{~b}(\mathrm{i}) . * \mathrm{Wq})))$

$\left(\mathrm{L}(7,3) *\left(\operatorname{sum}\left(\mathrm{g} 3322 \mathrm{a}(\mathrm{i}) .{ }^{*} \mathrm{Wq}\right)+\operatorname{sum}\left(\mathrm{g} 3322 \mathrm{~b}(\mathrm{i}) .{ }^{*} \mathrm{Wq}\right)+\operatorname{sum}(\mathrm{g} 2332 \mathrm{a}(\mathrm{i}) . * \mathrm{Wq})+\operatorname{sum}(\mathrm{g} 23\right.\right.$

$32 \mathrm{~b}(\mathrm{i}) \cdot * \mathrm{Wq}))$

$\left(\mathrm{L}(3,3) *\left(\operatorname{sum}\left(\mathrm{g} 3323 \mathrm{a}(\mathrm{i}) .{ }^{*} \mathrm{Wq}\right)+\operatorname{sum}(\mathrm{g} 3323 \mathrm{~b}(\mathrm{i}) . * \mathrm{Wq})+\operatorname{sum}(\mathrm{g} 2333 \mathrm{a}(\mathrm{i}) . * \mathrm{Wq})+\operatorname{sum}(\mathrm{g} 23\right.\right.$

$33 \mathrm{~b}(\mathrm{i}) * \mathrm{Wq})))) /(8 * \mathrm{pi})$;

S 3223

$\left(\left(\mathrm{L}(1,4) *\left(\operatorname{sum}\left(\mathrm{g} 3121 \mathrm{a}(\mathrm{i}) .{ }^{*} \mathrm{Wq}\right)+\operatorname{sum}\left(\mathrm{g} 3121 \mathrm{~b}(\mathrm{i}) .{ }^{*} \mathrm{Wq}\right)+\operatorname{sum}\left(\mathrm{g} 2131 \mathrm{a}(\mathrm{i}) .{ }^{*} \mathrm{Wq}\right)+\operatorname{sum}(\mathrm{g} 2\right.\right.\right.$ $131 \mathrm{~b}(\mathrm{i}) * \mathrm{Wq})))$

$\left(\mathrm{L}(6,4) *\left(\operatorname{sum}\left(\mathrm{g} 3122 \mathrm{a}(\mathrm{i}) .{ }^{*} \mathrm{Wq}\right)+\operatorname{sum}\left(\mathrm{g} 3122 \mathrm{~b}(\mathrm{i}) .{ }^{*} \mathrm{Wq}\right)+\operatorname{sum}\left(\mathrm{g} 2132 \mathrm{a}(\mathrm{i}) .{ }^{*} \mathrm{Wq}\right)+\operatorname{sum}(\mathrm{g} 21\right.\right.$ $\left.\left.\left.32 \mathrm{~b}(\mathrm{i}){ }^{*} \mathrm{Wq}\right)\right)\right)$

$\left(\mathrm{L}(8,4) *\left(\operatorname{sum}\left(\mathrm{g} 3123 \mathrm{a}(\mathrm{i}) .{ }^{*} \mathrm{Wq}\right)+\operatorname{sum}\left(\mathrm{g} 3123 \mathrm{~b}(\mathrm{i}) .{ }^{*} \mathrm{Wq}\right)+\operatorname{sum}\left(\mathrm{g} 2133 \mathrm{a}(\mathrm{i}) .{ }^{*} \mathrm{Wq}\right)+\operatorname{sum}(\mathrm{g} 21\right.\right.$

$33 \mathrm{~b}(\mathrm{i}) . * \mathrm{Wq})))$

$\left(\mathrm{L}(9,4) *\left(\operatorname{sum}(\mathrm{g} 3221 \mathrm{a}(\mathrm{i}) . * \mathrm{Wq})+\operatorname{sum}\left(\mathrm{g} 3221 \mathrm{~b}(\mathrm{i}) \cdot{ }^{*} \mathrm{Wq}\right)+\operatorname{sum}\left(\mathrm{g} 2231 \mathrm{a}(\mathrm{i}) \cdot{ }^{*} \mathrm{Wq}\right)+\operatorname{sum}(\mathrm{g} 22\right.\right.$

$31 \mathrm{~b}(\mathrm{i}) . * \mathrm{Wq})))$ 
$\left(\mathrm{L}(2,4) *\left(\operatorname{sum}\left(\mathrm{g} 3222 \mathrm{a}(\mathrm{i}) .{ }^{*} \mathrm{Wq}\right)+\operatorname{sum}\left(\mathrm{g} 3222 \mathrm{~b}(\mathrm{i}) \cdot{ }^{*} \mathrm{Wq}\right)+\operatorname{sum}\left(\mathrm{g} 2232 \mathrm{a}(\mathrm{i}) \cdot{ }^{*} \mathrm{Wq}\right)+\operatorname{sum}(\mathrm{g} 22\right.\right.$ $32 \mathrm{~b}(\mathrm{i}) . * \mathrm{Wq}))$ ) $\left(\mathrm{L}(4,4) *\left(\operatorname{sum}(\mathrm{g} 3223 \mathrm{a}(\mathrm{i}) . * \mathrm{Wq})+\operatorname{sum}\left(\mathrm{g} 3223 \mathrm{~b}(\mathrm{i}) \cdot{ }^{*} \mathrm{Wq}\right)+\operatorname{sum}\left(\mathrm{g} 2233 \mathrm{a}(\mathrm{i}) \cdot{ }^{*} \mathrm{Wq}\right)+\operatorname{sum}(\mathrm{g} 22\right.\right.$ $33 \mathrm{~b}(\mathrm{i}) . * \mathrm{Wq})))$ $\left(\mathrm{L}(5,4) *\left(\operatorname{sum}\left(\mathrm{g} 3321 \mathrm{a}(\mathrm{i}) .{ }^{*} \mathrm{Wq}\right)+\operatorname{sum}\left(\mathrm{g} 3321 \mathrm{~b}(\mathrm{i}) .{ }^{*} \mathrm{Wq}\right)+\operatorname{sum}(\mathrm{g} 2331 \mathrm{a}(\mathrm{i}) . * \mathrm{Wq})+\operatorname{sum}(\mathrm{g} 23\right.\right.$ $31 \mathrm{~b}(\mathrm{i}) . * \mathrm{Wq})))$ $\left(\mathrm{L}(7,4) *\left(\operatorname{sum}(\mathrm{g} 3322 \mathrm{a}(\mathrm{i}) . * \mathrm{Wq})+\operatorname{sum}\left(\mathrm{g} 3322 \mathrm{~b}(\mathrm{i}) \cdot{ }^{*} \mathrm{Wq}\right)+\operatorname{sum}\left(\mathrm{g} 2332 \mathrm{a}(\mathrm{i}) .{ }^{*} \mathrm{Wq}\right)+\operatorname{sum}(\mathrm{g} 23\right.\right.$ $32 \mathrm{~b}(\mathrm{i}) . * \mathrm{Wq})))$ $\left(\mathrm{L}(3,4) *\left(\operatorname{sum}\left(\mathrm{g} 3323 \mathrm{a}(\mathrm{i}) .{ }^{*} \mathrm{Wq}\right)+\operatorname{sum}\left(\mathrm{g} 3323 \mathrm{~b}(\mathrm{i}) .{ }^{*} \mathrm{Wq}\right)+\operatorname{sum}(\mathrm{g} 2333 \mathrm{a}(\mathrm{i}) . * \mathrm{Wq})+\operatorname{sum}(\mathrm{g} 23\right.\right.$ $\left.\left.\left.\left.33 \mathrm{~b}(\mathrm{i}) *{ }^{*} \mathrm{Wq}\right)\right)\right)\right) /(8 * \mathrm{pi})$;

$\mathrm{S} 3231$

$\left(\left(\mathrm{L}(1,5) *\left(\operatorname{sum}\left(\mathrm{g} 3121 \mathrm{a}(\mathrm{i}) .{ }^{*} \mathrm{Wq}\right)+\operatorname{sum}\left(\mathrm{g} 3121 \mathrm{~b}(\mathrm{i}) .{ }^{*} \mathrm{Wq}\right)+\operatorname{sum}\left(\mathrm{g} 2131 \mathrm{a}(\mathrm{i}) .{ }^{*} \mathrm{Wq}\right)+\operatorname{sum}(\mathrm{g} 2\right.\right.\right.$ $\left.\left.\left.131 \mathrm{~b}(\mathrm{i}) \cdot{ }^{*} \mathrm{Wq}\right)\right)\right)$

$\left(\mathrm{L}(6,5) *\left(\operatorname{sum}\left(\mathrm{g} 3122 \mathrm{a}(\mathrm{i}) .{ }^{*} \mathrm{Wq}\right)+\operatorname{sum}\left(\mathrm{g} 3122 \mathrm{~b}(\mathrm{i}) .{ }^{*} \mathrm{Wq}\right)+\operatorname{sum}(\mathrm{g} 2132 \mathrm{a}(\mathrm{i}) . * \mathrm{Wq})+\operatorname{sum}(\mathrm{g} 21\right.\right.$ $32 \mathrm{~b}(\mathrm{i}) . * \mathrm{Wq}))$ ) $\left(\mathrm{L}(8,5) *\left(\operatorname{sum}\left(\mathrm{g} 3123 \mathrm{a}(\mathrm{i}) .{ }^{*} \mathrm{Wq}\right)+\operatorname{sum}\left(\mathrm{g} 3123 \mathrm{~b}(\mathrm{i}) .{ }^{*} \mathrm{Wq}\right)+\operatorname{sum}(\mathrm{g} 2133 \mathrm{a}(\mathrm{i}) . * \mathrm{Wq})+\operatorname{sum}(\mathrm{g} 21\right.\right.$ $33 \mathrm{~b}(\mathrm{i}) . * \mathrm{Wq})))$ $\left(\mathrm{L}(9,5) *\left(\operatorname{sum}(\mathrm{g} 3221 \mathrm{a}(\mathrm{i}) . * \mathrm{Wq})+\operatorname{sum}\left(\mathrm{g} 3221 \mathrm{~b}(\mathrm{i}) .{ }^{*} \mathrm{Wq}\right)+\operatorname{sum}\left(\mathrm{g} 2231 \mathrm{a}(\mathrm{i}) \cdot{ }^{*} \mathrm{Wq}\right)+\operatorname{sum}(\mathrm{g} 22\right.\right.$ $31 \mathrm{~b}(\mathrm{i}) . * \mathrm{Wq})))$ $\left(\mathrm{L}(2,5) *\left(\operatorname{sum}\left(\mathrm{g} 3222 \mathrm{a}(\mathrm{i}) .{ }^{*} \mathrm{Wq}\right)+\operatorname{sum}\left(\mathrm{g} 3222 \mathrm{~b}(\mathrm{i}) .{ }^{*} \mathrm{Wq}\right)+\operatorname{sum}\left(\mathrm{g} 2232 \mathrm{a}(\mathrm{i}) .{ }^{*} \mathrm{Wq}\right)+\operatorname{sum}(\mathrm{g} 22\right.\right.$ $32 \mathrm{~b}(\mathrm{i}) . * \mathrm{Wq})))$ $\left(\mathrm{L}(4,5) *\left(\operatorname{sum}(\mathrm{g} 3223 \mathrm{a}(\mathrm{i}) . * \mathrm{Wq})+\operatorname{sum}\left(\mathrm{g} 3223 \mathrm{~b}(\mathrm{i}) \cdot{ }^{*} \mathrm{Wq}\right)+\operatorname{sum}\left(\mathrm{g} 2233 \mathrm{a}(\mathrm{i}) \cdot{ }^{*} \mathrm{Wq}\right)+\operatorname{sum}(\mathrm{g} 22\right.\right.$ $33 \mathrm{~b}(\mathrm{i}) . * \mathrm{Wq})))$ $\left(\mathrm{L}(5,5) *\left(\operatorname{sum}\left(\mathrm{g} 3321 \mathrm{a}(\mathrm{i}) .{ }^{*} \mathrm{Wq}\right)+\operatorname{sum}(\mathrm{g} 3321 \mathrm{~b}(\mathrm{i}) . * \mathrm{Wq})+\operatorname{sum}(\mathrm{g} 2331 \mathrm{a}(\mathrm{i}) . * \mathrm{Wq})+\operatorname{sum}(\mathrm{g} 23\right.\right.$ $31 \mathrm{~b}(\mathrm{i}) . * \mathrm{Wq})))$ $\left(\mathrm{L}(7,5) *\left(\operatorname{sum}\left(\mathrm{g} 3322 \mathrm{a}(\mathrm{i}) .{ }^{*} \mathrm{Wq}\right)+\operatorname{sum}\left(\mathrm{g} 3322 \mathrm{~b}(\mathrm{i}) .{ }^{*} \mathrm{Wq}\right)+\operatorname{sum}\left(\mathrm{g} 2332 \mathrm{a}(\mathrm{i}) .{ }^{*} \mathrm{Wq}\right)+\operatorname{sum}(\mathrm{g} 23\right.\right.$ $32 \mathrm{~b}(\mathrm{i}) . * \mathrm{Wq})))$ $\left(\mathrm{L}(3,5) *\left(\operatorname{sum}\left(\mathrm{g} 3323 \mathrm{a}(\mathrm{i}) .{ }^{*} \mathrm{Wq}\right)+\operatorname{sum}\left(\mathrm{g} 3323 \mathrm{~b}(\mathrm{i}) .{ }^{*} \mathrm{Wq}\right)+\operatorname{sum}\left(\mathrm{g} 2333 \mathrm{a}(\mathrm{i}) .{ }^{*} \mathrm{Wq}\right)+\operatorname{sum}(\mathrm{g} 23\right.\right.$ $33 \mathrm{~b}(\mathrm{i}) . * \mathrm{Wq})))) /(8 * \mathrm{pi})$;

$\mathrm{S} 3212$

$\left(\left(\mathrm{L}(1,6) *\left(\operatorname{sum}\left(\mathrm{g} 3121 \mathrm{a}(\mathrm{i}) .{ }^{*} \mathrm{Wq}\right)+\operatorname{sum}\left(\mathrm{g} 3121 \mathrm{~b}(\mathrm{i}) .{ }^{*} \mathrm{Wq}\right)+\operatorname{sum}\left(\mathrm{g} 2131 \mathrm{a}(\mathrm{i}) .{ }^{*} \mathrm{Wq}\right)+\operatorname{sum}(\mathrm{g} 2\right.\right.\right.$ $\left.\left.131 \mathrm{~b}(\mathrm{i}) \cdot{ }^{*} \mathrm{Wq}\right)\right)$ $\left(\mathrm{L}(6,6) *\left(\operatorname{sum}\left(\mathrm{g} 3122 \mathrm{a}(\mathrm{i}) .{ }^{*} \mathrm{Wq}\right)+\operatorname{sum}\left(\mathrm{g} 3122 \mathrm{~b}(\mathrm{i}) .{ }^{*} \mathrm{Wq}\right)+\operatorname{sum}(\mathrm{g} 2132 \mathrm{a}(\mathrm{i}) . * \mathrm{Wq})+\operatorname{sum}(\mathrm{g} 21\right.\right.$ $32 \mathrm{~b}(\mathrm{i}) . * \mathrm{Wq})))$ $\left(\mathrm{L}(8,6) *\left(\operatorname{sum}(\mathrm{g} 3123 \mathrm{a}(\mathrm{i}) . * \mathrm{Wq})+\operatorname{sum}\left(\mathrm{g} 3123 \mathrm{~b}(\mathrm{i}) \cdot{ }^{*} \mathrm{Wq}\right)+\operatorname{sum}(\mathrm{g} 2133 \mathrm{a}(\mathrm{i}) . * \mathrm{Wq})+\operatorname{sum}(\mathrm{g} 21\right.\right.$ $33 \mathrm{~b}(\mathrm{i}) \cdot * \mathrm{Wq}))$ $\left(\mathrm{L}(9,6) *\left(\operatorname{sum}\left(\mathrm{g} 3221 \mathrm{a}(\mathrm{i}) .{ }^{*} \mathrm{Wq}\right)+\operatorname{sum}\left(\mathrm{g} 3221 \mathrm{~b}(\mathrm{i}) .{ }^{*} \mathrm{Wq}\right)+\operatorname{sum}\left(\mathrm{g} 2231 \mathrm{a}(\mathrm{i}) \cdot{ }^{*} \mathrm{Wq}\right)+\operatorname{sum}(\mathrm{g} 22\right.\right.$ $31 \mathrm{~b}(\mathrm{i}) . * \mathrm{Wq})))$ $\left(\mathrm{L}(2,6) *\left(\operatorname{sum}\left(\mathrm{g} 3222 \mathrm{a}(\mathrm{i}) .{ }^{*} \mathrm{Wq}\right)+\operatorname{sum}\left(\mathrm{g} 3222 \mathrm{~b}(\mathrm{i}) .{ }^{*} \mathrm{Wq}\right)+\operatorname{sum}\left(\mathrm{g} 2232 \mathrm{a}(\mathrm{i}) .{ }^{*} \mathrm{Wq}\right)+\operatorname{sum}(\mathrm{g} 22\right.\right.$ $32 \mathrm{~b}(\mathrm{i}) . * \mathrm{Wq})))$ $\left(\mathrm{L}(4,6) *\left(\operatorname{sum}\left(\mathrm{g} 3223 \mathrm{a}(\mathrm{i}) .{ }^{*} \mathrm{Wq}\right)+\operatorname{sum}(\mathrm{g} 3223 \mathrm{~b}(\mathrm{i}) . * \mathrm{Wq})+\operatorname{sum}\left(\mathrm{g} 2233 \mathrm{a}(\mathrm{i}) .{ }^{*} \mathrm{Wq}\right)+\operatorname{sum}(\mathrm{g} 22\right.\right.$ $33 \mathrm{~b}(\mathrm{i}) . * \mathrm{Wq})))$ $\left(\mathrm{L}(5,6) *\left(\operatorname{sum}\left(\mathrm{g} 3321 \mathrm{a}(\mathrm{i}) .{ }^{*} \mathrm{Wq}\right)+\operatorname{sum}\left(\mathrm{g} 3321 \mathrm{~b}(\mathrm{i}) .{ }^{*} \mathrm{Wq}\right)+\operatorname{sum}\left(\mathrm{g} 2331 \mathrm{a}(\mathrm{i}) .{ }^{*} \mathrm{Wq}\right)+\operatorname{sum}(\mathrm{g} 23\right.\right.$ 
$31 \mathrm{~b}(\mathrm{i}) . * \mathrm{Wq})))$

$\left(\mathrm{L}(7,6) *\left(\operatorname{sum}\left(\mathrm{g} 3322 \mathrm{a}(\mathrm{i}) .{ }^{*} \mathrm{Wq}\right)+\operatorname{sum}\left(\mathrm{g} 3322 \mathrm{~b}(\mathrm{i}) .{ }^{*} \mathrm{Wq}\right)+\operatorname{sum}\left(\mathrm{g} 2332 \mathrm{a}(\mathrm{i}) .{ }^{*} \mathrm{Wq}\right)+\operatorname{sum}(\mathrm{g} 23\right.\right.$

$32 \mathrm{~b}(\mathrm{i}) . * \mathrm{Wq}))$ )

$\left(\mathrm{L}(3,6) *\left(\operatorname{sum}\left(\mathrm{g} 3323 \mathrm{a}(\mathrm{i}) .{ }^{*} \mathrm{Wq}\right)+\operatorname{sum}\left(\mathrm{g} 3323 \mathrm{~b}(\mathrm{i}) .{ }^{*} \mathrm{Wq}\right)+\operatorname{sum}\left(\mathrm{g} 2333 \mathrm{a}(\mathrm{i}) .{ }^{*} \mathrm{Wq}\right)+\operatorname{sum}(\mathrm{g} 23\right.\right.$

$\left.\left.\left.\left.33 \mathrm{~b}(\mathrm{i}) \cdot{ }^{*} \mathrm{Wq}\right)\right)\right)\right) /(8 * \mathrm{pi})$;

$\mathrm{S} 3232$

$\left(\left(\mathrm{L}(1,7) *\left(\operatorname{sum}\left(\mathrm{g} 3121 \mathrm{a}(\mathrm{i}) .{ }^{*} \mathrm{Wq}\right)+\operatorname{sum}\left(\mathrm{g} 3121 \mathrm{~b}(\mathrm{i}) .{ }^{*} \mathrm{Wq}\right)+\operatorname{sum}\left(\mathrm{g} 2131 \mathrm{a}(\mathrm{i}) .{ }^{*} \mathrm{Wq}\right)+\operatorname{sum}(\mathrm{g} 2\right.\right.\right.$ $131 \mathrm{~b}(\mathrm{i}) \cdot * \mathrm{Wq})))$

$\left(\mathrm{L}(6,7) *\left(\operatorname{sum}\left(\mathrm{g} 3122 \mathrm{a}(\mathrm{i}) .{ }^{*} \mathrm{Wq}\right)+\operatorname{sum}\left(\mathrm{g} 3122 \mathrm{~b}(\mathrm{i}) .{ }^{*} \mathrm{Wq}\right)+\operatorname{sum}(\mathrm{g} 2132 \mathrm{a}(\mathrm{i}) . * \mathrm{Wq})+\operatorname{sum}(\mathrm{g} 21\right.\right.$

$32 \mathrm{~b}(\mathrm{i}) . * \mathrm{Wq})))$

$\left(\mathrm{L}(8,7) *\left(\operatorname{sum}\left(\mathrm{g} 3123 \mathrm{a}(\mathrm{i}) .{ }^{*} \mathrm{Wq}\right)+\operatorname{sum}\left(\mathrm{g} 3123 \mathrm{~b}(\mathrm{i}) \cdot{ }^{*} \mathrm{Wq}\right)+\operatorname{sum}\left(\mathrm{g} 2133 \mathrm{a}(\mathrm{i}) .{ }^{*} \mathrm{Wq}\right)+\operatorname{sum}(\mathrm{g} 21\right.\right.$

$\left.\left.\left.33 \mathrm{~b}(\mathrm{i}) \cdot{ }^{*} \mathrm{Wq}\right)\right)\right)$

$\left(\mathrm{L}(9,7) *\left(\operatorname{sum}\left(\mathrm{g} 3221 \mathrm{a}(\mathrm{i}) \cdot{ }^{*} \mathrm{Wq}\right)+\operatorname{sum}\left(\mathrm{g} 3221 \mathrm{~b}(\mathrm{i}) .{ }^{*} \mathrm{Wq}\right)+\operatorname{sum}\left(\mathrm{g} 2231 \mathrm{a}(\mathrm{i}) \cdot{ }^{*} \mathrm{Wq}\right)+\operatorname{sum}(\mathrm{g} 22\right.\right.$

$31 \mathrm{~b}(\mathrm{i}) \cdot * \mathrm{Wq}))$

$\left(\mathrm{L}(2,7) *\left(\operatorname{sum}\left(\mathrm{g} 3222 \mathrm{a}(\mathrm{i}) .{ }^{*} \mathrm{Wq}\right)+\operatorname{sum}\left(\mathrm{g} 3222 \mathrm{~b}(\mathrm{i}) \cdot{ }^{*} \mathrm{Wq}\right)+\operatorname{sum}(\mathrm{g} 2232 \mathrm{a}(\mathrm{i}) . * \mathrm{Wq})+\operatorname{sum}(\mathrm{g} 22\right.\right.$

$32 \mathrm{~b}(\mathrm{i}) . * \mathrm{Wq})))$

$\left(\mathrm{L}(4,7) *\left(\operatorname{sum}\left(\mathrm{g} 3223 \mathrm{a}(\mathrm{i}) .{ }^{*} \mathrm{Wq}\right)+\operatorname{sum}\left(\mathrm{g} 3223 \mathrm{~b}(\mathrm{i}) .{ }^{*} \mathrm{Wq}\right)+\operatorname{sum}\left(\mathrm{g} 2233 \mathrm{a}(\mathrm{i}) .{ }^{*} \mathrm{Wq}\right)+\operatorname{sum}(\mathrm{g} 22\right.\right.$

$33 \mathrm{~b}(\mathrm{i}) \cdot * \mathrm{Wq}))$

$\left(\mathrm{L}(5,7) *\left(\operatorname{sum}\left(\mathrm{g} 3321 \mathrm{a}(\mathrm{i}) .{ }^{*} \mathrm{Wq}\right)+\operatorname{sum}\left(\mathrm{g} 3321 \mathrm{~b}(\mathrm{i}) .{ }^{*} \mathrm{Wq}\right)+\operatorname{sum}\left(\mathrm{g} 2331 \mathrm{a}(\mathrm{i}) .{ }^{*} \mathrm{Wq}\right)+\operatorname{sum}(\mathrm{g} 23\right.\right.$

$31 \mathrm{~b}(\mathrm{i}) . * \mathrm{Wq})))$

$\left(\mathrm{L}(7,7) *\left(\operatorname{sum}\left(\mathrm{g} 3322 \mathrm{a}(\mathrm{i}) .{ }^{*} \mathrm{Wq}\right)+\operatorname{sum}\left(\mathrm{g} 3322 \mathrm{~b}(\mathrm{i}) \cdot{ }^{*} \mathrm{Wq}\right)+\operatorname{sum}\left(\mathrm{g} 2332 \mathrm{a}(\mathrm{i}) .{ }^{*} \mathrm{Wq}\right)+\operatorname{sum}(\mathrm{g} 23\right.\right.$

$32 \mathrm{~b}(\mathrm{i}) . * \mathrm{Wq}))$

$\left(\mathrm{L}(3,7) *\left(\operatorname{sum}(\mathrm{g} 3323 \mathrm{a}(\mathrm{i}) . * \mathrm{Wq})+\operatorname{sum}\left(\mathrm{g} 3323 \mathrm{~b}(\mathrm{i}) \cdot{ }^{*} \mathrm{Wq}\right)+\operatorname{sum}(\mathrm{g} 2333 \mathrm{a}(\mathrm{i}) . * \mathrm{Wq})+\operatorname{sum}(\mathrm{g} 23\right.\right.$

$33 \mathrm{~b}(\mathrm{i}) * \mathrm{Wq})))) /(8 * \mathrm{pi})$;

$\mathrm{S} 3213$

$\left(\left(\mathrm{L}(1,8) *\left(\operatorname{sum}\left(\mathrm{g} 3121 \mathrm{a}(\mathrm{i}) .{ }^{*} \mathrm{Wq}\right)+\operatorname{sum}\left(\mathrm{g} 3121 \mathrm{~b}(\mathrm{i}) .{ }^{*} \mathrm{Wq}\right)+\operatorname{sum}\left(\mathrm{g} 2131 \mathrm{a}(\mathrm{i}) .{ }^{*} \mathrm{Wq}\right)+\operatorname{sum}(\mathrm{g} 2\right.\right.\right.$ $\left.\left.\left.131 \mathrm{~b}(\mathrm{i}) *{ }^{*} \mathrm{Wq}\right)\right)\right)$

$\left(\mathrm{L}(6,8) *\left(\operatorname{sum}\left(\mathrm{g} 3122 \mathrm{a}(\mathrm{i}) .{ }^{*} \mathrm{Wq}\right)+\operatorname{sum}\left(\mathrm{g} 3122 \mathrm{~b}(\mathrm{i}) .{ }^{*} \mathrm{Wq}\right)+\operatorname{sum}(\mathrm{g} 2132 \mathrm{a}(\mathrm{i}) . * \mathrm{Wq})+\operatorname{sum}(\mathrm{g} 21\right.\right.$

$32 \mathrm{~b}(\mathrm{i}) . * \mathrm{Wq})))$

$\left(\mathrm{L}(8,8) *\left(\operatorname{sum}\left(\mathrm{g} 3123 \mathrm{a}(\mathrm{i}) .{ }^{*} \mathrm{Wq}\right)+\operatorname{sum}\left(\mathrm{g} 3123 \mathrm{~b}(\mathrm{i}) \cdot{ }^{*} \mathrm{Wq}\right)+\operatorname{sum}(\mathrm{g} 2133 \mathrm{a}(\mathrm{i}) . * \mathrm{Wq})+\operatorname{sum}(\mathrm{g} 21\right.\right.$

$33 \mathrm{~b}(\mathrm{i}) . * \mathrm{Wq})))$

$\left(\mathrm{L}(9,8) *\left(\operatorname{sum}\left(\mathrm{g} 3221 \mathrm{a}(\mathrm{i}) .{ }^{*} \mathrm{Wq}\right)+\operatorname{sum}\left(\mathrm{g} 3221 \mathrm{~b}(\mathrm{i}) .{ }^{*} \mathrm{Wq}\right)+\operatorname{sum}(\mathrm{g} 2231 \mathrm{a}(\mathrm{i}) . * \mathrm{Wq})+\operatorname{sum}(\mathrm{g} 22\right.\right.$

$31 \mathrm{~b}(\mathrm{i}) . * \mathrm{Wq})))$

$\left(\mathrm{L}(2,8) *\left(\operatorname{sum}\left(\mathrm{g} 3222 \mathrm{a}(\mathrm{i}) .{ }^{*} \mathrm{Wq}\right)+\operatorname{sum}\left(\mathrm{g} 3222 \mathrm{~b}(\mathrm{i}) \cdot{ }^{*} \mathrm{Wq}\right)+\operatorname{sum}(\mathrm{g} 2232 \mathrm{a}(\mathrm{i}) . * \mathrm{Wq})+\operatorname{sum}(\mathrm{g} 22\right.\right.$

$32 \mathrm{~b}(\mathrm{i}) . * \mathrm{Wq})))$

$\left(\mathrm{L}(4,8) *\left(\operatorname{sum}\left(\mathrm{g} 3223 \mathrm{a}(\mathrm{i}) .{ }^{*} \mathrm{Wq}\right)+\operatorname{sum}\left(\mathrm{g} 3223 \mathrm{~b}(\mathrm{i}) \cdot{ }^{*} \mathrm{Wq}\right)+\operatorname{sum}\left(\mathrm{g} 2233 \mathrm{a}(\mathrm{i}) \cdot{ }^{*} \mathrm{Wq}\right)+\operatorname{sum}(\mathrm{g} 22\right.\right.$

$33 \mathrm{~b}(\mathrm{i}) . * \mathrm{Wq})))$

$\left(\mathrm{L}(5,8) *\left(\operatorname{sum}\left(\mathrm{g} 3321 \mathrm{a}(\mathrm{i}) .{ }^{*} \mathrm{Wq}\right)+\operatorname{sum}\left(\mathrm{g} 3321 \mathrm{~b}(\mathrm{i}) .{ }^{*} \mathrm{Wq}\right)+\operatorname{sum}\left(\mathrm{g} 2331 \mathrm{a}(\mathrm{i}) .{ }^{*} \mathrm{Wq}\right)+\operatorname{sum}(\mathrm{g} 23\right.\right.$

$31 \mathrm{~b}(\mathrm{i}) . * \mathrm{Wq}))$

$\left(\mathrm{L}(7,8) *\left(\operatorname{sum}\left(\mathrm{g} 3322 \mathrm{a}(\mathrm{i}) .{ }^{*} \mathrm{Wq}\right)+\operatorname{sum}\left(\mathrm{g} 3322 \mathrm{~b}(\mathrm{i}) \cdot{ }^{*} \mathrm{Wq}\right)+\operatorname{sum}(\mathrm{g} 2332 \mathrm{a}(\mathrm{i}) . * \mathrm{Wq})+\operatorname{sum}(\mathrm{g} 23\right.\right.$

$32 \mathrm{~b}(\mathrm{i}) . * \mathrm{Wq}))$

$\left(\mathrm{L}(3,8) *\left(\operatorname{sum}(\mathrm{g} 3323 \mathrm{a}(\mathrm{i}) . * \mathrm{Wq})+\operatorname{sum}\left(\mathrm{g} 3323 \mathrm{~b}(\mathrm{i}) \cdot{ }^{*} \mathrm{Wq}\right)+\operatorname{sum}\left(\mathrm{g} 2333 \mathrm{a}(\mathrm{i}) \cdot{ }^{*} \mathrm{Wq}\right)+\operatorname{sum}(\mathrm{g} 23\right.\right.$

$\left.\left.\left.\left.33 \mathrm{~b}(\mathrm{i}){ }^{*} \mathrm{Wq}\right)\right)\right)\right) /(8 * \mathrm{pi})$; 
S 3221

$\left(\left(\mathrm{L}(1,9) *\left(\operatorname{sum}\left(\mathrm{g} 3121 \mathrm{a}(\mathrm{i}) .{ }^{*} \mathrm{Wq}\right)+\operatorname{sum}\left(\mathrm{g} 3121 \mathrm{~b}(\mathrm{i}) .{ }^{*} \mathrm{Wq}\right)+\operatorname{sum}(\mathrm{g} 2131 \mathrm{a}(\mathrm{i}) . * \mathrm{Wq})+\operatorname{sum}(\mathrm{g} 2\right.\right.\right.$ $\left.\left.\left.131 \mathrm{~b}(\mathrm{i}) \cdot{ }^{*} \mathrm{Wq}\right)\right)\right)$

$\left(\mathrm{L}(6,9) *\left(\operatorname{sum}(\mathrm{g} 3122 \mathrm{a}(\mathrm{i}) . * \mathrm{Wq})+\operatorname{sum}\left(\mathrm{g} 3122 \mathrm{~b}(\mathrm{i}) \cdot{ }^{*} \mathrm{Wq}\right)+\operatorname{sum}\left(\mathrm{g} 2132 \mathrm{a}(\mathrm{i}) \cdot{ }^{*} \mathrm{Wq}\right)+\operatorname{sum}(\mathrm{g} 21\right.\right.$ $\left.\left.\left.32 \mathrm{~b}(\mathrm{i}) \cdot{ }^{*} \mathrm{Wq}\right)\right)\right)$

$\left(\mathrm{L}(8,9) *\left(\operatorname{sum}\left(\mathrm{g} 3123 \mathrm{a}(\mathrm{i}) .{ }^{*} \mathrm{Wq}\right)+\operatorname{sum}\left(\mathrm{g} 3123 \mathrm{~b}(\mathrm{i}) .{ }^{*} \mathrm{Wq}\right)+\operatorname{sum}\left(\mathrm{g} 2133 \mathrm{a}(\mathrm{i}) .{ }^{*} \mathrm{Wq}\right)+\operatorname{sum}(\mathrm{g} 21\right.\right.$ $\left.\left.\left.33 \mathrm{~b}(\mathrm{i}) \cdot{ }^{*} \mathrm{Wq}\right)\right)\right)$

$\left(\mathrm{L}(9,9) *\left(\operatorname{sum}\left(\mathrm{g} 3221 \mathrm{a}(\mathrm{i}) .{ }^{*} \mathrm{Wq}\right)+\operatorname{sum}\left(\mathrm{g} 3221 \mathrm{~b}(\mathrm{i}) .{ }^{*} \mathrm{Wq}\right)+\operatorname{sum}\left(\mathrm{g} 2231 \mathrm{a}(\mathrm{i}) .{ }^{*} \mathrm{Wq}\right)+\operatorname{sum}(\mathrm{g} 22\right.\right.$

$31 \mathrm{~b}(\mathrm{i}) . * \mathrm{Wq})))$

$\left(\mathrm{L}(2,9) *\left(\operatorname{sum}(\mathrm{g} 3222 \mathrm{a}(\mathrm{i}) . * \mathrm{Wq})+\operatorname{sum}\left(\mathrm{g} 3222 \mathrm{~b}(\mathrm{i}) \cdot{ }^{*} \mathrm{Wq}\right)+\operatorname{sum}\left(\mathrm{g} 2232 \mathrm{a}(\mathrm{i}) \cdot{ }^{*} \mathrm{Wq}\right)+\operatorname{sum}(\mathrm{g} 22\right.\right.$

$\left.\left.\left.32 \mathrm{~b}(\mathrm{i}){ }^{*} \mathrm{Wq}\right)\right)\right)$

$\left(\mathrm{L}(4,9) *\left(\operatorname{sum}\left(\mathrm{g} 3223 \mathrm{a}(\mathrm{i}) \cdot{ }^{*} \mathrm{Wq}\right)+\operatorname{sum}\left(\mathrm{g} 3223 \mathrm{~b}(\mathrm{i}) \cdot{ }^{*} \mathrm{Wq}\right)+\operatorname{sum}\left(\mathrm{g} 2233 \mathrm{a}(\mathrm{i}) \cdot{ }^{*} \mathrm{Wq}\right)+\operatorname{sum}(\mathrm{g} 22\right.\right.$

$\left.\left.\left.33 \mathrm{~b}(\mathrm{i}) \cdot{ }^{*} \mathrm{Wq}\right)\right)\right)$

$\left(\mathrm{L}(5,9) *\left(\operatorname{sum}\left(\mathrm{g} 3321 \mathrm{a}(\mathrm{i}) .{ }^{*} \mathrm{Wq}\right)+\operatorname{sum}\left(\mathrm{g} 3321 \mathrm{~b}(\mathrm{i}) .{ }^{*} \mathrm{Wq}\right)+\operatorname{sum}\left(\mathrm{g} 2331 \mathrm{a}(\mathrm{i}) .{ }^{*} \mathrm{Wq}\right)+\operatorname{sum}(\mathrm{g} 23\right.\right.$

$31 \mathrm{~b}(\mathrm{i}) . * \mathrm{Wq}))$ )

$\left(\mathrm{L}(7,9) *\left(\operatorname{sum}(\mathrm{g} 3322 \mathrm{a}(\mathrm{i}) . * \mathrm{Wq})+\operatorname{sum}\left(\mathrm{g} 3322 \mathrm{~b}(\mathrm{i}) \cdot{ }^{*} \mathrm{Wq}\right)+\operatorname{sum}\left(\mathrm{g} 2332 \mathrm{a}(\mathrm{i}) \cdot{ }^{*} \mathrm{Wq}\right)+\operatorname{sum}(\mathrm{g} 23\right.\right.$

$32 \mathrm{~b}(\mathrm{i}) . * \mathrm{Wq}))$ )

$\left(\mathrm{L}(3,9) *\left(\operatorname{sum}\left(\mathrm{g} 3323 \mathrm{a}(\mathrm{i}) .{ }^{*} \mathrm{Wq}\right)+\operatorname{sum}\left(\mathrm{g} 3323 \mathrm{~b}(\mathrm{i}) .{ }^{*} \mathrm{Wq}\right)+\operatorname{sum}(\mathrm{g} 2333 \mathrm{a}(\mathrm{i}) . * \mathrm{Wq})+\operatorname{sum}(\mathrm{g} 23\right.\right.$

$\left.\left.\left.\left.33 \mathrm{~b}(\mathrm{i}){ }^{*} \mathrm{Wq}\right)\right)\right)\right) /(8 * \mathrm{pi})$;

S1311

$\left(\left(\mathrm{L}(1,1) *\left(\operatorname{sum}\left(\mathrm{g} 1131 \mathrm{a}(\mathrm{i}) .{ }^{*} \mathrm{Wq}\right)+\operatorname{sum}\left(\mathrm{g} 1131 \mathrm{~b}(\mathrm{i}) .{ }^{*} \mathrm{Wq}\right)+\operatorname{sum}(\mathrm{g} 3111 \mathrm{a}(\mathrm{i}) . * \mathrm{Wq})+\operatorname{sum}(\mathrm{g} 3\right.\right.\right.$ $111 \mathrm{~b}(\mathrm{i}) \cdot * \mathrm{Wq})))$

$\left(\mathrm{L}(6,1) *\left(\operatorname{sum}\left(\mathrm{g} 1132 \mathrm{a}(\mathrm{i}) .{ }^{*} \mathrm{Wq}\right)+\operatorname{sum}\left(\mathrm{g} 1132 \mathrm{~b}(\mathrm{i}) \cdot{ }^{*} \mathrm{Wq}\right)+\operatorname{sum}(\mathrm{g} 3112 \mathrm{a}(\mathrm{i}) . * \mathrm{Wq})+\operatorname{sum}(\mathrm{g} 31\right.\right.$

$12 \mathrm{~b}(\mathrm{i}) . * \mathrm{Wq})))$

$\left(\mathrm{L}(8,1) *\left(\operatorname{sum}\left(\mathrm{g} 1133 \mathrm{a}(\mathrm{i}) .{ }^{*} \mathrm{Wq}\right)+\operatorname{sum}\left(\mathrm{g} 1133 \mathrm{~b}(\mathrm{i}) .{ }^{*} \mathrm{Wq}\right)+\operatorname{sum}\left(\mathrm{g} 3113 \mathrm{a}(\mathrm{i}) .{ }^{*} \mathrm{Wq}\right)+\operatorname{sum}(\mathrm{g} 31\right.\right.$

$13 \mathrm{~b}(\mathrm{i}) . * \mathrm{Wq})))$

$\left(\mathrm{L}(9,1) *\left(\operatorname{sum}\left(\mathrm{g} 1231 \mathrm{a}(\mathrm{i}) .{ }^{*} \mathrm{Wq}\right)+\operatorname{sum}\left(\mathrm{g} 1231 \mathrm{~b}(\mathrm{i}) .{ }^{*} \mathrm{Wq}\right)+\operatorname{sum}\left(\mathrm{g} 3211 \mathrm{a}(\mathrm{i}) .{ }^{*} \mathrm{Wq}\right)+\operatorname{sum}(\mathrm{g} 32\right.\right.$

$11 \mathrm{~b}(\mathrm{i}) . * \mathrm{Wq})))$

$\left(\mathrm{L}(2,1) *\left(\operatorname{sum}\left(\mathrm{g} 1232 \mathrm{a}(\mathrm{i}) .{ }^{*} \mathrm{Wq}\right)+\operatorname{sum}\left(\mathrm{g} 1232 \mathrm{~b}(\mathrm{i}) .{ }^{*} \mathrm{Wq}\right)+\operatorname{sum}\left(\mathrm{g} 3212 \mathrm{a}(\mathrm{i}) .{ }^{*} \mathrm{Wq}\right)+\operatorname{sum}(\mathrm{g} 32\right.\right.$

$12 \mathrm{~b}(\mathrm{i}) . * \mathrm{Wq})))$

$\left(\mathrm{L}(4,1) *\left(\operatorname{sum}\left(\mathrm{g} 1233 \mathrm{a}(\mathrm{i}) .{ }^{*} \mathrm{Wq}\right)+\operatorname{sum}\left(\mathrm{g} 1233 \mathrm{~b}(\mathrm{i}) .{ }^{*} \mathrm{Wq}\right)+\operatorname{sum}\left(\mathrm{g} 3213 \mathrm{a}(\mathrm{i}) .{ }^{*} \mathrm{Wq}\right)+\operatorname{sum}(\mathrm{g} 32\right.\right.$

$13 \mathrm{~b}(\mathrm{i}) . * \mathrm{Wq}))$

$\left(\mathrm{L}(5,1) *\left(\operatorname{sum}\left(\mathrm{g} 1331 \mathrm{a}(\mathrm{i}) .{ }^{*} \mathrm{Wq}\right)+\operatorname{sum}\left(\mathrm{g} 1331 \mathrm{~b}(\mathrm{i}) .{ }^{*} \mathrm{Wq}\right)+\operatorname{sum}\left(\mathrm{g} 3311 \mathrm{a}(\mathrm{i}) .{ }^{*} \mathrm{Wq}\right)+\operatorname{sum}(\mathrm{g} 33\right.\right.$

$11 \mathrm{~b}(\mathrm{i}) . * \mathrm{Wq})))$

$(\mathrm{L}(7,1) *(\operatorname{sum}(\mathrm{g} 1332 \mathrm{a}(\mathrm{i}) . * \mathrm{Wq})+\operatorname{sum}(\mathrm{g} 1332 \mathrm{~b}(\mathrm{i}) . * \mathrm{Wq})+\operatorname{sum}(\mathrm{g} 3312 \mathrm{a}(\mathrm{i}) . * \mathrm{Wq})+\operatorname{sum}(\mathrm{g} 33$

$\left.\left.\left.12 \mathrm{~b}(\mathrm{i}) \cdot{ }^{*} \mathrm{Wq}\right)\right)\right)$

$\left(\mathrm{L}(3,1) *\left(\operatorname{sum}\left(\mathrm{g} 1333 \mathrm{a}(\mathrm{i}) .{ }^{*} \mathrm{Wq}\right)+\operatorname{sum}\left(\mathrm{g} 1333 \mathrm{~b}(\mathrm{i}) .{ }^{*} \mathrm{Wq}\right)+\operatorname{sum}\left(\mathrm{g} 3313 \mathrm{a}(\mathrm{i}) .{ }^{*} \mathrm{Wq}\right)+\operatorname{sum}(\mathrm{g} 33\right.\right.$

$\left.\left.\left.\left.13 \mathrm{~b}(\mathrm{i}) \cdot{ }^{*} \mathrm{Wq}\right)\right)\right)\right) /(8 * \mathrm{pi})$;

$\mathrm{S} 1322$

$\left(\left(\mathrm{L}(1,2) *\left(\operatorname{sum}\left(\mathrm{g} 1131 \mathrm{a}(\mathrm{i}) .{ }^{*} \mathrm{Wq}\right)+\operatorname{sum}\left(\mathrm{g} 1131 \mathrm{~b}(\mathrm{i}) .{ }^{*} \mathrm{Wq}\right)+\operatorname{sum}\left(\mathrm{g} 3111 \mathrm{a}(\mathrm{i}) .{ }^{*} \mathrm{Wq}\right)+\operatorname{sum}(\mathrm{g} 3\right.\right.\right.$ $111 \mathrm{~b}(\mathrm{i}) * \mathrm{Wq})))$

$\left(\mathrm{L}(6,2) *\left(\operatorname{sum}\left(\mathrm{g} 1132 \mathrm{a}(\mathrm{i}) .{ }^{*} \mathrm{Wq}\right)+\operatorname{sum}\left(\mathrm{g} 1132 \mathrm{~b}(\mathrm{i}) \cdot{ }^{*} \mathrm{Wq}\right)+\operatorname{sum}(\mathrm{g} 3112 \mathrm{a}(\mathrm{i}) . * \mathrm{Wq})+\operatorname{sum}(\mathrm{g} 31\right.\right.$ 
$12 \mathrm{~b}(\mathrm{i}) . * \mathrm{Wq})))$

$\left(\mathrm{L}(8,2) *\left(\operatorname{sum}\left(\mathrm{g} 1133 \mathrm{a}(\mathrm{i}) .{ }^{*} \mathrm{Wq}\right)+\operatorname{sum}\left(\mathrm{g} 1133 \mathrm{~b}(\mathrm{i}) \cdot{ }^{*} \mathrm{Wq}\right)+\operatorname{sum}\left(\mathrm{g} 3113 \mathrm{a}(\mathrm{i}) .{ }^{*} \mathrm{Wq}\right)+\operatorname{sum}(\mathrm{g} 31\right.\right.$

$\left.\left.\left.13 \mathrm{~b}(\mathrm{i}) *{ }^{*} \mathrm{Wq}\right)\right)\right)$

$\left(\mathrm{L}(9,2) *\left(\operatorname{sum}\left(\mathrm{g} 1231 \mathrm{a}(\mathrm{i}) .{ }^{*} \mathrm{Wq}\right)+\operatorname{sum}\left(\mathrm{g} 1231 \mathrm{~b}(\mathrm{i}) \cdot{ }^{*} \mathrm{Wq}\right)+\operatorname{sum}\left(\mathrm{g} 3211 \mathrm{a}(\mathrm{i}) \cdot{ }^{*} \mathrm{Wq}\right)+\operatorname{sum}(\mathrm{g} 32\right.\right.$

$\left.\left.\left.11 \mathrm{~b}(\mathrm{i}) \cdot{ }^{*} \mathrm{Wq}\right)\right)\right)$

$\left(\mathrm{L}(2,2) *\left(\operatorname{sum}\left(\mathrm{g} 1232 \mathrm{a}(\mathrm{i}) \cdot{ }^{*} \mathrm{Wq}\right)+\operatorname{sum}\left(\mathrm{g} 1232 \mathrm{~b}(\mathrm{i}) \cdot{ }^{*} \mathrm{Wq}\right)+\operatorname{sum}\left(\mathrm{g} 3212 \mathrm{a}(\mathrm{i}) \cdot{ }^{*} \mathrm{Wq}\right)+\operatorname{sum}(\mathrm{g} 32\right.\right.$

$12 \mathrm{~b}(\mathrm{i}) . * \mathrm{Wq})))$

$\left(\mathrm{L}(4,2) *\left(\operatorname{sum}\left(\mathrm{g} 1233 \mathrm{a}(\mathrm{i}) .{ }^{*} \mathrm{Wq}\right)+\operatorname{sum}\left(\mathrm{g} 1233 \mathrm{~b}(\mathrm{i}) .{ }^{*} \mathrm{Wq}\right)+\operatorname{sum}\left(\mathrm{g} 3213 \mathrm{a}(\mathrm{i}) .{ }^{*} \mathrm{Wq}\right)+\operatorname{sum}(\mathrm{g} 32\right.\right.$

$13 \mathrm{~b}(\mathrm{i}) . * \mathrm{Wq}))$

$\left(\mathrm{L}(5,2) *\left(\operatorname{sum}\left(\mathrm{g} 1331 \mathrm{a}(\mathrm{i}) .{ }^{*} \mathrm{Wq}\right)+\operatorname{sum}\left(\mathrm{g} 1331 \mathrm{~b}(\mathrm{i}) .{ }^{*} \mathrm{Wq}\right)+\operatorname{sum}\left(\mathrm{g} 3311 \mathrm{a}(\mathrm{i}) .{ }^{*} \mathrm{Wq}\right)+\operatorname{sum}(\mathrm{g} 33\right.\right.$

$11 \mathrm{~b}(\mathrm{i}) . * \mathrm{Wq})))$

$\left(\mathrm{L}(7,2) *\left(\operatorname{sum}\left(\mathrm{g} 1332 \mathrm{a}(\mathrm{i}) .{ }^{*} \mathrm{Wq}\right)+\operatorname{sum}\left(\mathrm{g} 1332 \mathrm{~b}(\mathrm{i}) \cdot{ }^{*} \mathrm{Wq}\right)+\operatorname{sum}\left(\mathrm{g} 3312 \mathrm{a}(\mathrm{i}) .{ }^{*} \mathrm{Wq}\right)+\operatorname{sum}(\mathrm{g} 33\right.\right.$

$\left.\left.\left.12 \mathrm{~b}(\mathrm{i}) \cdot{ }^{*} \mathrm{Wq}\right)\right)\right)$

$\left(\mathrm{L}(3,2) *\left(\operatorname{sum}\left(\mathrm{g} 1333 \mathrm{a}(\mathrm{i}) .{ }^{*} \mathrm{Wq}\right)+\operatorname{sum}\left(\mathrm{g} 1333 \mathrm{~b}(\mathrm{i}) \cdot{ }^{*} \mathrm{Wq}\right)+\operatorname{sum}\left(\mathrm{g} 3313 \mathrm{a}(\mathrm{i}) .{ }^{*} \mathrm{Wq}\right)+\operatorname{sum}(\mathrm{g} 33\right.\right.$

$\left.\left.\left.\left.13 \mathrm{~b}(\mathrm{i}){ }^{*} \mathrm{Wq}\right)\right)\right)\right) /(8 * \mathrm{pi})$;

S1333

$((\mathrm{L}(1,3) *(\operatorname{sum}(\mathrm{g} 1131 \mathrm{a}(\mathrm{i}) . * \mathrm{Wq})+\operatorname{sum}(\mathrm{g} 1131 \mathrm{~b}(\mathrm{i}) . * \mathrm{Wq})+\operatorname{sum}(\mathrm{g} 3111 \mathrm{a}(\mathrm{i}) . * \mathrm{Wq})+\operatorname{sum}(\mathrm{g} 3$ $\left.\left.\left.111 \mathrm{~b}(\mathrm{i}) *{ }^{*} \mathrm{Wq}\right)\right)\right)$

$\left(\mathrm{L}(6,3) *\left(\operatorname{sum}\left(\mathrm{g} 1132 \mathrm{a}(\mathrm{i}) .{ }^{*} \mathrm{Wq}\right)+\operatorname{sum}\left(\mathrm{g} 1132 \mathrm{~b}(\mathrm{i}) .{ }^{*} \mathrm{Wq}\right)+\operatorname{sum}(\mathrm{g} 3112 \mathrm{a}(\mathrm{i}) . * \mathrm{Wq})+\operatorname{sum}(\mathrm{g} 31\right.\right.$

$12 \mathrm{~b}(\mathrm{i}) . * \mathrm{Wq})))$

$\left(\mathrm{L}(8,3) *\left(\operatorname{sum}\left(\mathrm{g} 1133 \mathrm{a}(\mathrm{i}) .{ }^{*} \mathrm{Wq}\right)+\operatorname{sum}\left(\mathrm{g} 1133 \mathrm{~b}(\mathrm{i}) \cdot{ }^{*} \mathrm{Wq}\right)+\operatorname{sum}\left(\mathrm{g} 3113 \mathrm{a}(\mathrm{i}) .{ }^{*} \mathrm{Wq}\right)+\operatorname{sum}(\mathrm{g} 31\right.\right.$

$13 \mathrm{~b}(\mathrm{i}) . * \mathrm{Wq})))$

$\left(\mathrm{L}(9,3) *\left(\operatorname{sum}\left(\mathrm{g} 1231 \mathrm{a}(\mathrm{i}) .{ }^{*} \mathrm{Wq}\right)+\operatorname{sum}\left(\mathrm{g} 1231 \mathrm{~b}(\mathrm{i}) .{ }^{*} \mathrm{Wq}\right)+\operatorname{sum}\left(\mathrm{g} 3211 \mathrm{a}(\mathrm{i}) .{ }^{*} \mathrm{Wq}\right)+\operatorname{sum}(\mathrm{g} 32\right.\right.$

$11 \mathrm{~b}(\mathrm{i}) . * \mathrm{Wq})))$

$\left(\mathrm{L}(2,3) *\left(\operatorname{sum}\left(\mathrm{g} 1232 \mathrm{a}(\mathrm{i}) \cdot{ }^{*} \mathrm{Wq}\right)+\operatorname{sum}\left(\mathrm{g} 1232 \mathrm{~b}(\mathrm{i}) \cdot{ }^{*} \mathrm{Wq}\right)+\operatorname{sum}\left(\mathrm{g} 3212 \mathrm{a}(\mathrm{i}) \cdot{ }^{*} \mathrm{Wq}\right)+\operatorname{sum}(\mathrm{g} 32\right.\right.$

$12 \mathrm{~b}(\mathrm{i}) \cdot * \mathrm{Wq}))$

$\left(\mathrm{L}(4,3) *\left(\operatorname{sum}\left(\mathrm{g} 1233 \mathrm{a}(\mathrm{i}) .{ }^{*} \mathrm{Wq}\right)+\operatorname{sum}\left(\mathrm{g} 1233 \mathrm{~b}(\mathrm{i}) .{ }^{*} \mathrm{Wq}\right)+\operatorname{sum}\left(\mathrm{g} 3213 \mathrm{a}(\mathrm{i}) .{ }^{*} \mathrm{Wq}\right)+\operatorname{sum}(\mathrm{g} 32\right.\right.$

$13 \mathrm{~b}(\mathrm{i}) . * \mathrm{Wq}))$

$\left(\mathrm{L}(5,3) *\left(\operatorname{sum}\left(\mathrm{g} 1331 \mathrm{a}(\mathrm{i}) .{ }^{*} \mathrm{Wq}\right)+\operatorname{sum}\left(\mathrm{g} 1331 \mathrm{~b}(\mathrm{i}) .{ }^{*} \mathrm{Wq}\right)+\operatorname{sum}\left(\mathrm{g} 3311 \mathrm{a}(\mathrm{i}) .{ }^{*} \mathrm{Wq}\right)+\operatorname{sum}(\mathrm{g} 33\right.\right.$

$11 \mathrm{~b}(\mathrm{i}) . * \mathrm{Wq})))$

$\left(\mathrm{L}(7,3) *\left(\operatorname{sum}\left(\mathrm{g} 1332 \mathrm{a}(\mathrm{i}) .{ }^{*} \mathrm{Wq}\right)+\operatorname{sum}\left(\mathrm{g} 1332 \mathrm{~b}(\mathrm{i}) .{ }^{*} \mathrm{Wq}\right)+\operatorname{sum}(\mathrm{g} 3312 \mathrm{a}(\mathrm{i}) . * \mathrm{Wq})+\operatorname{sum}(\mathrm{g} 33\right.\right.$

$12 \mathrm{~b}(\mathrm{i}) . * \mathrm{Wq})))$

$\left(\mathrm{L}(3,3) *\left(\operatorname{sum}\left(\mathrm{g} 1333 \mathrm{a}(\mathrm{i}) .{ }^{*} \mathrm{Wq}\right)+\operatorname{sum}\left(\mathrm{g} 1333 \mathrm{~b}(\mathrm{i}) .{ }^{*} \mathrm{Wq}\right)+\operatorname{sum}\left(\mathrm{g} 3313 \mathrm{a}(\mathrm{i}) .{ }^{*} \mathrm{Wq}\right)+\operatorname{sum}(\mathrm{g} 33\right.\right.$

$\left.\left.\left.\left.13 \mathrm{~b}(\mathrm{i}) \cdot{ }^{*} \mathrm{Wq}\right)\right)\right)\right) /(8 * \mathrm{pi})$;

$\mathrm{S} 1323$

$\left(\left(\mathrm{L}(1,4) *\left(\operatorname{sum}(\mathrm{g} 1131 \mathrm{a}(\mathrm{i}) . * \mathrm{Wq})+\operatorname{sum}(\mathrm{g} 1131 \mathrm{~b}(\mathrm{i}) . * \mathrm{Wq})+\operatorname{sum}\left(\mathrm{g} 3111 \mathrm{a}(\mathrm{i}) .{ }^{*} \mathrm{Wq}\right)+\operatorname{sum}(\mathrm{g} 3\right.\right.\right.$ $111 \mathrm{~b}(\mathrm{i}) * \mathrm{Wq})))$

$\left(\mathrm{L}(6,4) *\left(\operatorname{sum}\left(\mathrm{g} 1132 \mathrm{a}(\mathrm{i}) .{ }^{*} \mathrm{Wq}\right)+\operatorname{sum}\left(\mathrm{g} 1132 \mathrm{~b}(\mathrm{i}) .{ }^{*} \mathrm{Wq}\right)+\operatorname{sum}\left(\mathrm{g} 3112 \mathrm{a}(\mathrm{i}) .{ }^{*} \mathrm{Wq}\right)+\operatorname{sum}(\mathrm{g} 31\right.\right.$

$12 \mathrm{~b}(\mathrm{i}) . * \mathrm{Wq})))$

$\left(\mathrm{L}(8,4) *\left(\operatorname{sum}\left(\mathrm{g} 1133 \mathrm{a}(\mathrm{i}) .{ }^{*} \mathrm{Wq}\right)+\operatorname{sum}\left(\mathrm{g} 1133 \mathrm{~b}(\mathrm{i}) .{ }^{*} \mathrm{Wq}\right)+\operatorname{sum}\left(\mathrm{g} 3113 \mathrm{a}(\mathrm{i}) .{ }^{*} \mathrm{Wq}\right)+\operatorname{sum}(\mathrm{g} 31\right.\right.$

$13 \mathrm{~b}(\mathrm{i}) . * \mathrm{Wq}))$

$\left(\mathrm{L}(9,4) *\left(\operatorname{sum}\left(\mathrm{g} 1231 \mathrm{a}(\mathrm{i}) .{ }^{*} \mathrm{Wq}\right)+\operatorname{sum}\left(\mathrm{g} 1231 \mathrm{~b}(\mathrm{i}) \cdot{ }^{*} \mathrm{Wq}\right)+\operatorname{sum}\left(\mathrm{g} 3211 \mathrm{a}(\mathrm{i}) \cdot{ }^{*} \mathrm{Wq}\right)+\operatorname{sum}(\mathrm{g} 32\right.\right.$

$\left.\left.\left.11 \mathrm{~b}(\mathrm{i}) .{ }^{*} \mathrm{Wq}\right)\right)\right)$ 
$\left(\mathrm{L}(2,4) *\left(\operatorname{sum}\left(\mathrm{g} 1232 \mathrm{a}(\mathrm{i}) \cdot{ }^{*} \mathrm{Wq}\right)+\operatorname{sum}\left(\mathrm{g} 1232 \mathrm{~b}(\mathrm{i}) \cdot{ }^{*} \mathrm{Wq}\right)+\operatorname{sum}\left(\mathrm{g} 3212 \mathrm{a}(\mathrm{i}) \cdot{ }^{*} \mathrm{Wq}\right)+\operatorname{sum}(\mathrm{g} 32\right.\right.$ $12 \mathrm{~b}(\mathrm{i}) . * \mathrm{Wq}))$ ) $\left(\mathrm{L}(4,4) *\left(\operatorname{sum}(\mathrm{g} 1233 \mathrm{a}(\mathrm{i}) . * \mathrm{Wq})+\operatorname{sum}\left(\mathrm{g} 1233 \mathrm{~b}(\mathrm{i}) \cdot{ }^{*} \mathrm{Wq}\right)+\operatorname{sum}\left(\mathrm{g} 3213 \mathrm{a}(\mathrm{i}) \cdot{ }^{*} \mathrm{Wq}\right)+\operatorname{sum}(\mathrm{g} 32\right.\right.$ $13 \mathrm{~b}(\mathrm{i}) . * \mathrm{Wq})))$ $\left(\mathrm{L}(5,4) *\left(\operatorname{sum}\left(\mathrm{g} 1331 \mathrm{a}(\mathrm{i}) .{ }^{*} \mathrm{Wq}\right)+\operatorname{sum}\left(\mathrm{g} 1331 \mathrm{~b}(\mathrm{i}) .{ }^{*} \mathrm{Wq}\right)+\operatorname{sum}\left(\mathrm{g} 3311 \mathrm{a}(\mathrm{i}) .{ }^{*} \mathrm{Wq}\right)+\operatorname{sum}(\mathrm{g} 33\right.\right.$ $11 \mathrm{~b}(\mathrm{i}) . * \mathrm{Wq})))$ $\left(\mathrm{L}(7,4) *\left(\operatorname{sum}(\mathrm{g} 1332 \mathrm{a}(\mathrm{i}) . * \mathrm{Wq})+\operatorname{sum}\left(\mathrm{g} 1332 \mathrm{~b}(\mathrm{i}) \cdot{ }^{*} \mathrm{Wq}\right)+\operatorname{sum}\left(\mathrm{g} 3312 \mathrm{a}(\mathrm{i}) .{ }^{*} \mathrm{Wq}\right)+\operatorname{sum}(\mathrm{g} 33\right.\right.$ $12 \mathrm{~b}(\mathrm{i}) . * \mathrm{Wq})))$ $\left(\mathrm{L}(3,4) *\left(\operatorname{sum}\left(\mathrm{g} 1333 \mathrm{a}(\mathrm{i}) .{ }^{*} \mathrm{Wq}\right)+\operatorname{sum}\left(\mathrm{g} 1333 \mathrm{~b}(\mathrm{i}) .{ }^{*} \mathrm{Wq}\right)+\operatorname{sum}\left(\mathrm{g} 3313 \mathrm{a}(\mathrm{i}) .{ }^{*} \mathrm{Wq}\right)+\operatorname{sum}(\mathrm{g} 33\right.\right.$ $\left.\left.\left.\left.13 \mathrm{~b}(\mathrm{i}){ }^{*} \mathrm{Wq}\right)\right)\right)\right) /(8 * \mathrm{pi})$;

$\mathrm{S} 1331$

$\left(\left(\mathrm{L}(1,5) *\left(\operatorname{sum}\left(\mathrm{g} 1131 \mathrm{a}(\mathrm{i}) .{ }^{*} \mathrm{Wq}\right)+\operatorname{sum}\left(\mathrm{g} 1131 \mathrm{~b}(\mathrm{i}) .{ }^{*} \mathrm{Wq}\right)+\operatorname{sum}\left(\mathrm{g} 3111 \mathrm{a}(\mathrm{i}) .{ }^{*} \mathrm{Wq}\right)+\operatorname{sum}(\mathrm{g} 3\right.\right.\right.$ $\left.\left.\left.111 \mathrm{~b}(\mathrm{i}) \cdot{ }^{*} \mathrm{Wq}\right)\right)\right)$ $\left(\mathrm{L}(6,5) *\left(\operatorname{sum}\left(\mathrm{g} 1132 \mathrm{a}(\mathrm{i}) .{ }^{*} \mathrm{Wq}\right)+\operatorname{sum}\left(\mathrm{g} 1132 \mathrm{~b}(\mathrm{i}) \cdot{ }^{*} \mathrm{Wq}\right)+\operatorname{sum}\left(\mathrm{g} 3112 \mathrm{a}(\mathrm{i}) .{ }^{*} \mathrm{Wq}\right)+\operatorname{sum}(\mathrm{g} 31\right.\right.$ $12 \mathrm{~b}(\mathrm{i}) . * \mathrm{Wq}))$ ) $\left(\mathrm{L}(8,5) *\left(\operatorname{sum}\left(\mathrm{g} 1133 \mathrm{a}(\mathrm{i}) .{ }^{*} \mathrm{Wq}\right)+\operatorname{sum}\left(\mathrm{g} 1133 \mathrm{~b}(\mathrm{i}) .{ }^{*} \mathrm{Wq}\right)+\operatorname{sum}\left(\mathrm{g} 3113 \mathrm{a}(\mathrm{i}) .{ }^{*} \mathrm{Wq}\right)+\operatorname{sum}(\mathrm{g} 31\right.\right.$ $13 \mathrm{~b}(\mathrm{i}) . * \mathrm{Wq})))$ $\left(\mathrm{L}(9,5) *\left(\operatorname{sum}\left(\mathrm{g} 1231 \mathrm{a}(\mathrm{i}) .{ }^{*} \mathrm{Wq}\right)+\operatorname{sum}\left(\mathrm{g} 1231 \mathrm{~b}(\mathrm{i}) .{ }^{*} \mathrm{Wq}\right)+\operatorname{sum}\left(\mathrm{g} 3211 \mathrm{a}(\mathrm{i}) \cdot{ }^{*} \mathrm{Wq}\right)+\operatorname{sum}(\mathrm{g} 32\right.\right.$ $11 \mathrm{~b}(\mathrm{i}) . * \mathrm{Wq})))$ $\left(\mathrm{L}(2,5) *\left(\operatorname{sum}\left(\mathrm{g} 1232 \mathrm{a}(\mathrm{i}) .{ }^{*} \mathrm{Wq}\right)+\operatorname{sum}\left(\mathrm{g} 1232 \mathrm{~b}(\mathrm{i}) \cdot{ }^{*} \mathrm{Wq}\right)+\operatorname{sum}\left(\mathrm{g} 3212 \mathrm{a}(\mathrm{i}) \cdot{ }^{*} \mathrm{Wq}\right)+\operatorname{sum}(\mathrm{g} 32\right.\right.$ $12 \mathrm{~b}(\mathrm{i}) . * \mathrm{Wq})))$ $\left(\mathrm{L}(4,5) *\left(\operatorname{sum}\left(\mathrm{g} 1233 \mathrm{a}(\mathrm{i}) .{ }^{*} \mathrm{Wq}\right)+\operatorname{sum}\left(\mathrm{g} 1233 \mathrm{~b}(\mathrm{i}) \cdot{ }^{*} \mathrm{Wq}\right)+\operatorname{sum}\left(\mathrm{g} 3213 \mathrm{a}(\mathrm{i}) \cdot{ }^{*} \mathrm{Wq}\right)+\operatorname{sum}(\mathrm{g} 32\right.\right.$ $13 \mathrm{~b}(\mathrm{i}) . * \mathrm{Wq}))$ $\left(\mathrm{L}(5,5) *\left(\operatorname{sum}\left(\mathrm{g} 1331 \mathrm{a}(\mathrm{i}) .{ }^{*} \mathrm{Wq}\right)+\operatorname{sum}\left(\mathrm{g} 1331 \mathrm{~b}(\mathrm{i}) \cdot{ }^{*} \mathrm{Wq}\right)+\operatorname{sum}\left(\mathrm{g} 3311 \mathrm{a}(\mathrm{i}) .{ }^{*} \mathrm{Wq}\right)+\operatorname{sum}(\mathrm{g} 33\right.\right.$ $11 \mathrm{~b}(\mathrm{i}) . * \mathrm{Wq})))$ $\left(\mathrm{L}(7,5) *\left(\operatorname{sum}\left(\mathrm{g} 1332 \mathrm{a}(\mathrm{i}) .{ }^{*} \mathrm{Wq}\right)+\operatorname{sum}\left(\mathrm{g} 1332 \mathrm{~b}(\mathrm{i}) .{ }^{*} \mathrm{Wq}\right)+\operatorname{sum}\left(\mathrm{g} 3312 \mathrm{a}(\mathrm{i}) .{ }^{*} \mathrm{Wq}\right)+\operatorname{sum}(\mathrm{g} 33\right.\right.$ $12 \mathrm{~b}(\mathrm{i}) . * \mathrm{Wq})))$ $\left(\mathrm{L}(3,5) *\left(\operatorname{sum}\left(\mathrm{g} 1333 \mathrm{a}(\mathrm{i}) .{ }^{*} \mathrm{Wq}\right)+\operatorname{sum}\left(\mathrm{g} 1333 \mathrm{~b}(\mathrm{i}) .{ }^{*} \mathrm{Wq}\right)+\operatorname{sum}\left(\mathrm{g} 3313 \mathrm{a}(\mathrm{i}) .{ }^{*} \mathrm{Wq}\right)+\operatorname{sum}(\mathrm{g} 33\right.\right.$ $\left.\left.\left.\left.13 \mathrm{~b}(\mathrm{i}) \cdot{ }^{*} \mathrm{Wq}\right)\right)\right)\right) /(8 * \mathrm{pi})$;

$\mathrm{S} 1312$

$\left(\left(\mathrm{L}(1,6) *\left(\operatorname{sum}\left(\mathrm{g} 1131 \mathrm{a}(\mathrm{i}) .{ }^{*} \mathrm{Wq}\right)+\operatorname{sum}\left(\mathrm{g} 1131 \mathrm{~b}(\mathrm{i}) .{ }^{*} \mathrm{Wq}\right)+\operatorname{sum}\left(\mathrm{g} 3111 \mathrm{a}(\mathrm{i}) .{ }^{*} \mathrm{Wq}\right)+\operatorname{sum}(\mathrm{g} 3\right.\right.\right.$ $111 \mathrm{~b}(\mathrm{i}) * \mathrm{Wq})))$ $\left(\mathrm{L}(6,6) *\left(\operatorname{sum}\left(\mathrm{g} 1132 \mathrm{a}(\mathrm{i}) .{ }^{*} \mathrm{Wq}\right)+\operatorname{sum}\left(\mathrm{g} 1132 \mathrm{~b}(\mathrm{i}) \cdot{ }^{*} \mathrm{Wq}\right)+\operatorname{sum}(\mathrm{g} 3112 \mathrm{a}(\mathrm{i}) . * \mathrm{Wq})+\operatorname{sum}(\mathrm{g} 31\right.\right.$ $12 \mathrm{~b}(\mathrm{i}) \cdot * \mathrm{Wq})))$ $\left(\mathrm{L}(8,6) *\left(\operatorname{sum}\left(\mathrm{g} 1133 \mathrm{a}(\mathrm{i}) .{ }^{*} \mathrm{Wq}\right)+\operatorname{sum}\left(\mathrm{g} 1133 \mathrm{~b}(\mathrm{i}) \cdot{ }^{*} \mathrm{Wq}\right)+\operatorname{sum}\left(\mathrm{g} 3113 \mathrm{a}(\mathrm{i}) \cdot{ }^{*} \mathrm{Wq}\right)+\operatorname{sum}(\mathrm{g} 31\right.\right.$ $13 \mathrm{~b}(\mathrm{i}) . * \mathrm{Wq})))$ $\left(\mathrm{L}(9,6) *\left(\operatorname{sum}\left(\mathrm{g} 1231 \mathrm{a}(\mathrm{i}) .{ }^{*} \mathrm{Wq}\right)+\operatorname{sum}\left(\mathrm{g} 1231 \mathrm{~b}(\mathrm{i}) .{ }^{*} \mathrm{Wq}\right)+\operatorname{sum}\left(\mathrm{g} 3211 \mathrm{a}(\mathrm{i}) \cdot{ }^{*} \mathrm{Wq}\right)+\operatorname{sum}(\mathrm{g} 32\right.\right.$ $\left.\left.\left.11 \mathrm{~b}(\mathrm{i}) \cdot{ }^{*} \mathrm{Wq}\right)\right)\right)$ $\left(\mathrm{L}(2,6) *\left(\operatorname{sum}\left(\mathrm{g} 1232 \mathrm{a}(\mathrm{i}) .{ }^{*} \mathrm{Wq}\right)+\operatorname{sum}\left(\mathrm{g} 1232 \mathrm{~b}(\mathrm{i}) \cdot{ }^{*} \mathrm{Wq}\right)+\operatorname{sum}\left(\mathrm{g} 3212 \mathrm{a}(\mathrm{i}) .{ }^{*} \mathrm{Wq}\right)+\operatorname{sum}(\mathrm{g} 32\right.\right.$ $12 \mathrm{~b}(\mathrm{i}) . * \mathrm{Wq})))$ $\left(\mathrm{L}(4,6) *\left(\operatorname{sum}\left(\mathrm{g} 1233 \mathrm{a}(\mathrm{i}) .{ }^{*} \mathrm{Wq}\right)+\operatorname{sum}\left(\mathrm{g} 1233 \mathrm{~b}(\mathrm{i}) \cdot{ }^{*} \mathrm{Wq}\right)+\operatorname{sum}\left(\mathrm{g} 3213 \mathrm{a}(\mathrm{i}) \cdot{ }^{*} \mathrm{Wq}\right)+\operatorname{sum}(\mathrm{g} 32\right.\right.$ $13 \mathrm{~b}(\mathrm{i}) . * \mathrm{Wq})))$ $\left(\mathrm{L}(5,6) *\left(\operatorname{sum}\left(\mathrm{g} 1331 \mathrm{a}(\mathrm{i}) .{ }^{*} \mathrm{Wq}\right)+\operatorname{sum}\left(\mathrm{g} 1331 \mathrm{~b}(\mathrm{i}) \cdot{ }^{*} \mathrm{Wq}\right)+\operatorname{sum}\left(\mathrm{g} 3311 \mathrm{a}(\mathrm{i}) .{ }^{*} \mathrm{Wq}\right)+\operatorname{sum}(\mathrm{g} 33\right.\right.$ 
$11 \mathrm{~b}(\mathrm{i}) . * \mathrm{Wq})))$

$\left(\mathrm{L}(7,6) *\left(\operatorname{sum}\left(\mathrm{g} 1332 \mathrm{a}(\mathrm{i}) .{ }^{*} \mathrm{Wq}\right)+\operatorname{sum}\left(\mathrm{g} 1332 \mathrm{~b}(\mathrm{i}) .{ }^{*} \mathrm{Wq}\right)+\operatorname{sum}\left(\mathrm{g} 3312 \mathrm{a}(\mathrm{i}) .{ }^{*} \mathrm{Wq}\right)+\operatorname{sum}(\mathrm{g} 33\right.\right.$

$12 \mathrm{~b}(\mathrm{i}) . * \mathrm{Wq})))$

$\left(\mathrm{L}(3,6) *\left(\operatorname{sum}\left(\mathrm{g} 1333 \mathrm{a}(\mathrm{i}) .{ }^{*} \mathrm{Wq}\right)+\operatorname{sum}\left(\mathrm{g} 1333 \mathrm{~b}(\mathrm{i}) .{ }^{*} \mathrm{Wq}\right)+\operatorname{sum}\left(\mathrm{g} 3313 \mathrm{a}(\mathrm{i}) .{ }^{*} \mathrm{Wq}\right)+\operatorname{sum}(\mathrm{g} 33\right.\right.$

$\left.\left.\left.\left.13 \mathrm{~b}(\mathrm{i}) \cdot{ }^{*} \mathrm{Wq}\right)\right)\right)\right) /(8 * \mathrm{pi})$;

$\mathrm{S} 1332$

$\left(\left(\mathrm{L}(1,7) *\left(\operatorname{sum}\left(\mathrm{g} 1131 \mathrm{a}(\mathrm{i}) .{ }^{*} \mathrm{Wq}\right)+\operatorname{sum}\left(\mathrm{g} 1131 \mathrm{~b}(\mathrm{i}) .{ }^{*} \mathrm{Wq}\right)+\operatorname{sum}\left(\mathrm{g} 3111 \mathrm{a}(\mathrm{i}) .{ }^{*} \mathrm{Wq}\right)+\operatorname{sum}(\mathrm{g} 3\right.\right.\right.$ $\left.\left.\left.111 \mathrm{~b}(\mathrm{i}) \cdot{ }^{*} \mathrm{Wq}\right)\right)\right)$

$\left(\mathrm{L}(6,7) *\left(\operatorname{sum}\left(\mathrm{g} 1132 \mathrm{a}(\mathrm{i}) .{ }^{*} \mathrm{Wq}\right)+\operatorname{sum}\left(\mathrm{g} 1132 \mathrm{~b}(\mathrm{i}) .{ }^{*} \mathrm{Wq}\right)+\operatorname{sum}(\mathrm{g} 3112 \mathrm{a}(\mathrm{i}) . * \mathrm{Wq})+\operatorname{sum}(\mathrm{g} 31\right.\right.$

$12 \mathrm{~b}(\mathrm{i}) . * \mathrm{Wq})))$

$\left(\mathrm{L}(8,7) *\left(\operatorname{sum}\left(\mathrm{g} 1133 \mathrm{a}(\mathrm{i}) .{ }^{*} \mathrm{Wq}\right)+\operatorname{sum}\left(\mathrm{g} 1133 \mathrm{~b}(\mathrm{i}) \cdot{ }^{*} \mathrm{Wq}\right)+\operatorname{sum}\left(\mathrm{g} 3113 \mathrm{a}(\mathrm{i}) .{ }^{*} \mathrm{Wq}\right)+\operatorname{sum}(\mathrm{g} 31\right.\right.$

$13 \mathrm{~b}(\mathrm{i}) . * \mathrm{Wq})))$

$\left(\mathrm{L}(9,7) *\left(\operatorname{sum}\left(\mathrm{g} 1231 \mathrm{a}(\mathrm{i}) .{ }^{*} \mathrm{Wq}\right)+\operatorname{sum}\left(\mathrm{g} 1231 \mathrm{~b}(\mathrm{i}) \cdot{ }^{*} \mathrm{Wq}\right)+\operatorname{sum}\left(\mathrm{g} 3211 \mathrm{a}(\mathrm{i}) .{ }^{*} \mathrm{Wq}\right)+\operatorname{sum}(\mathrm{g} 32\right.\right.$

$11 \mathrm{~b}(\mathrm{i}) . * \mathrm{Wq})))$

$\left(\mathrm{L}(2,7) *\left(\operatorname{sum}\left(\mathrm{g} 1232 \mathrm{a}(\mathrm{i}) .{ }^{*} \mathrm{Wq}\right)+\operatorname{sum}\left(\mathrm{g} 1232 \mathrm{~b}(\mathrm{i}) \cdot{ }^{*} \mathrm{Wq}\right)+\operatorname{sum}\left(\mathrm{g} 3212 \mathrm{a}(\mathrm{i}) .{ }^{*} \mathrm{Wq}\right)+\operatorname{sum}(\mathrm{g} 32\right.\right.$

$12 \mathrm{~b}(\mathrm{i}) . * \mathrm{Wq})))$

$\left(\mathrm{L}(4,7) *\left(\operatorname{sum}\left(\mathrm{g} 1233 \mathrm{a}(\mathrm{i}) .{ }^{*} \mathrm{Wq}\right)+\operatorname{sum}\left(\mathrm{g} 1233 \mathrm{~b}(\mathrm{i}) \cdot{ }^{*} \mathrm{Wq}\right)+\operatorname{sum}\left(\mathrm{g} 3213 \mathrm{a}(\mathrm{i}) .{ }^{*} \mathrm{Wq}\right)+\operatorname{sum}(\mathrm{g} 32\right.\right.$

$\left.\left.\left.13 \mathrm{~b}(\mathrm{i}) \cdot{ }^{*} \mathrm{Wq}\right)\right)\right)$

$\left(\mathrm{L}(5,7) *\left(\operatorname{sum}\left(\mathrm{g} 1331 \mathrm{a}(\mathrm{i}) .{ }^{*} \mathrm{Wq}\right)+\operatorname{sum}\left(\mathrm{g} 1331 \mathrm{~b}(\mathrm{i}) .{ }^{*} \mathrm{Wq}\right)+\operatorname{sum}\left(\mathrm{g} 3311 \mathrm{a}(\mathrm{i}) .{ }^{*} \mathrm{Wq}\right)+\operatorname{sum}(\mathrm{g} 33\right.\right.$

$11 \mathrm{~b}(\mathrm{i}) . * \mathrm{Wq})))$

$\left(\mathrm{L}(7,7) *\left(\operatorname{sum}\left(\mathrm{g} 1332 \mathrm{a}(\mathrm{i}) .{ }^{*} \mathrm{Wq}\right)+\operatorname{sum}\left(\mathrm{g} 1332 \mathrm{~b}(\mathrm{i}) \cdot{ }^{*} \mathrm{Wq}\right)+\operatorname{sum}\left(\mathrm{g} 3312 \mathrm{a}(\mathrm{i}) .{ }^{*} \mathrm{Wq}\right)+\operatorname{sum}(\mathrm{g} 33\right.\right.$

$12 \mathrm{~b}(\mathrm{i}) . * \mathrm{Wq})))$

$\left(\mathrm{L}(3,7) *\left(\operatorname{sum}(\mathrm{g} 1333 \mathrm{a}(\mathrm{i}) . * \mathrm{Wq})+\operatorname{sum}\left(\mathrm{g} 1333 \mathrm{~b}(\mathrm{i}) \cdot{ }^{*} \mathrm{Wq}\right)+\operatorname{sum}(\mathrm{g} 3313 \mathrm{a}(\mathrm{i}) . * \mathrm{Wq})+\operatorname{sum}(\mathrm{g} 33\right.\right.$

$\left.\left.\left.\left.13 \mathrm{~b}(\mathrm{i}) *{ }^{*} \mathrm{Wq}\right)\right)\right)\right) /(8 * \mathrm{pi})$;

$\mathrm{S} 1313$

$\left(\left(\mathrm{L}(1,8) *\left(\operatorname{sum}\left(\mathrm{g} 1131 \mathrm{a}(\mathrm{i}) .{ }^{*} \mathrm{Wq}\right)+\operatorname{sum}\left(\mathrm{g} 1131 \mathrm{~b}(\mathrm{i}) .{ }^{*} \mathrm{Wq}\right)+\operatorname{sum}\left(\mathrm{g} 3111 \mathrm{a}(\mathrm{i}) .{ }^{*} \mathrm{Wq}\right)+\operatorname{sum}(\mathrm{g} 3\right.\right.\right.$

$111 \mathrm{~b}(\mathrm{i}) * \mathrm{Wq})))$

$\left(\mathrm{L}(6,8) *\left(\operatorname{sum}\left(\mathrm{g} 1132 \mathrm{a}(\mathrm{i}) .{ }^{*} \mathrm{Wq}\right)+\operatorname{sum}\left(\mathrm{g} 1132 \mathrm{~b}(\mathrm{i}) \cdot{ }^{*} \mathrm{Wq}\right)+\operatorname{sum}\left(\mathrm{g} 3112 \mathrm{a}(\mathrm{i}) .{ }^{*} \mathrm{Wq}\right)+\operatorname{sum}(\mathrm{g} 31\right.\right.$

$12 \mathrm{~b}(\mathrm{i}) . * \mathrm{Wq}))$ )

$\left(\mathrm{L}(8,8) *\left(\operatorname{sum}\left(\mathrm{g} 1133 \mathrm{a}(\mathrm{i}) .{ }^{*} \mathrm{Wq}\right)+\operatorname{sum}\left(\mathrm{g} 1133 \mathrm{~b}(\mathrm{i}) .{ }^{*} \mathrm{Wq}\right)+\operatorname{sum}\left(\mathrm{g} 3113 \mathrm{a}(\mathrm{i}) .{ }^{*} \mathrm{Wq}\right)+\operatorname{sum}(\mathrm{g} 31\right.\right.$

$13 \mathrm{~b}(\mathrm{i}) \cdot * \mathrm{Wq}))$

$\left(\mathrm{L}(9,8) *\left(\operatorname{sum}\left(\mathrm{g} 1231 \mathrm{a}(\mathrm{i}) .{ }^{*} \mathrm{Wq}\right)+\operatorname{sum}\left(\mathrm{g} 1231 \mathrm{~b}(\mathrm{i}) .{ }^{*} \mathrm{Wq}\right)+\operatorname{sum}\left(\mathrm{g} 3211 \mathrm{a}(\mathrm{i}) .{ }^{*} \mathrm{Wq}\right)+\operatorname{sum}(\mathrm{g} 32\right.\right.$

$11 \mathrm{~b}(\mathrm{i}) . * \mathrm{Wq}))$

$\left(\mathrm{L}(2,8) *\left(\operatorname{sum}\left(\mathrm{g} 1232 \mathrm{a}(\mathrm{i}) .{ }^{*} \mathrm{Wq}\right)+\operatorname{sum}\left(\mathrm{g} 1232 \mathrm{~b}(\mathrm{i}) \cdot{ }^{*} \mathrm{Wq}\right)+\operatorname{sum}\left(\mathrm{g} 3212 \mathrm{a}(\mathrm{i}) .{ }^{*} \mathrm{Wq}\right)+\operatorname{sum}(\mathrm{g} 32\right.\right.$

$12 \mathrm{~b}(\mathrm{i}) . * \mathrm{Wq})))$

$\left(\mathrm{L}(4,8) *\left(\operatorname{sum}\left(\mathrm{g} 1233 \mathrm{a}(\mathrm{i}) .{ }^{*} \mathrm{Wq}\right)+\operatorname{sum}\left(\mathrm{g} 1233 \mathrm{~b}(\mathrm{i}) .{ }^{*} \mathrm{Wq}\right)+\operatorname{sum}\left(\mathrm{g} 3213 \mathrm{a}(\mathrm{i}) .{ }^{*} \mathrm{Wq}\right)+\operatorname{sum}(\mathrm{g} 32\right.\right.$

$\left.\left.\left.13 \mathrm{~b}(\mathrm{i}) \cdot{ }^{*} \mathrm{Wq}\right)\right)\right)$

$\left(\mathrm{L}(5,8) *\left(\operatorname{sum}\left(\mathrm{g} 1331 \mathrm{a}(\mathrm{i}) .{ }^{*} \mathrm{Wq}\right)+\operatorname{sum}\left(\mathrm{g} 1331 \mathrm{~b}(\mathrm{i}) .{ }^{*} \mathrm{Wq}\right)+\operatorname{sum}\left(\mathrm{g} 3311 \mathrm{a}(\mathrm{i}) .{ }^{*} \mathrm{Wq}\right)+\operatorname{sum}(\mathrm{g} 33\right.\right.$

$11 \mathrm{~b}(\mathrm{i}) . * \mathrm{Wq})))$

$\left(\mathrm{L}(7,8) *\left(\operatorname{sum}\left(\mathrm{g} 1332 \mathrm{a}(\mathrm{i}) .{ }^{*} \mathrm{Wq}\right)+\operatorname{sum}\left(\mathrm{g} 1332 \mathrm{~b}(\mathrm{i}) \cdot{ }^{*} \mathrm{Wq}\right)+\operatorname{sum}(\mathrm{g} 3312 \mathrm{a}(\mathrm{i}) . * \mathrm{Wq})+\operatorname{sum}(\mathrm{g} 33\right.\right.$

$12 \mathrm{~b}(\mathrm{i}) . * \mathrm{Wq})))$

$\left(\mathrm{L}(3,8) *\left(\operatorname{sum}\left(\mathrm{g} 1333 \mathrm{a}(\mathrm{i}) .{ }^{*} \mathrm{Wq}\right)+\operatorname{sum}\left(\mathrm{g} 1333 \mathrm{~b}(\mathrm{i}) \cdot{ }^{*} \mathrm{Wq}\right)+\operatorname{sum}\left(\mathrm{g} 3313 \mathrm{a}(\mathrm{i}) \cdot{ }^{*} \mathrm{Wq}\right)+\operatorname{sum}(\mathrm{g} 33\right.\right.$

$\left.\left.\left.\left.13 \mathrm{~b}(\mathrm{i}){ }^{*} \mathrm{Wq}\right)\right)\right)\right) /(8 * \mathrm{pi})$; 
S1321

$\left(\left(\mathrm{L}(1,9) *\left(\operatorname{sum}\left(\mathrm{g} 1131 \mathrm{a}(\mathrm{i}) .{ }^{*} \mathrm{Wq}\right)+\operatorname{sum}\left(\mathrm{g} 1131 \mathrm{~b}(\mathrm{i}) \cdot{ }^{*} \mathrm{Wq}\right)+\operatorname{sum}\left(\mathrm{g} 3111 \mathrm{a}(\mathrm{i}) .{ }^{*} \mathrm{Wq}\right)+\operatorname{sum}(\mathrm{g} 3\right.\right.\right.$ $111 b(\mathrm{i}) \cdot * \mathrm{Wq})))$

$\left(\mathrm{L}(6,9) *\left(\operatorname{sum}\left(\mathrm{g} 1132 \mathrm{a}(\mathrm{i}) .{ }^{*} \mathrm{Wq}\right)+\operatorname{sum}\left(\mathrm{g} 1132 \mathrm{~b}(\mathrm{i}) \cdot{ }^{*} \mathrm{Wq}\right)+\operatorname{sum}\left(\mathrm{g} 3112 \mathrm{a}(\mathrm{i}) .{ }^{*} \mathrm{Wq}\right)+\operatorname{sum}(\mathrm{g} 31\right.\right.$ $12 \mathrm{~b}(\mathrm{i}) . * \mathrm{Wq})))$

$\left(\mathrm{L}(8,9) *\left(\operatorname{sum}\left(\mathrm{g} 1133 \mathrm{a}(\mathrm{i}) .{ }^{*} \mathrm{Wq}\right)+\operatorname{sum}\left(\mathrm{g} 1133 \mathrm{~b}(\mathrm{i}) .{ }^{*} \mathrm{Wq}\right)+\operatorname{sum}\left(\mathrm{g} 3113 \mathrm{a}(\mathrm{i}) .{ }^{*} \mathrm{Wq}\right)+\operatorname{sum}(\mathrm{g} 31\right.\right.$ $13 \mathrm{~b}(\mathrm{i}) . * \mathrm{Wq})))$

$\left(\mathrm{L}(9,9) *\left(\operatorname{sum}\left(\mathrm{g} 1231 \mathrm{a}(\mathrm{i}) .{ }^{*} \mathrm{Wq}\right)+\operatorname{sum}\left(\mathrm{g} 1231 \mathrm{~b}(\mathrm{i}) \cdot{ }^{*} \mathrm{Wq}\right)+\operatorname{sum}\left(\mathrm{g} 3211 \mathrm{a}(\mathrm{i}) .{ }^{*} \mathrm{Wq}\right)+\operatorname{sum}(\mathrm{g} 32\right.\right.$ $11 \mathrm{~b}(\mathrm{i}) . * \mathrm{Wq})))$

$\left(\mathrm{L}(2,9) *\left(\operatorname{sum}(\mathrm{g} 1232 \mathrm{a}(\mathrm{i}) . * \mathrm{Wq})+\operatorname{sum}\left(\mathrm{g} 1232 \mathrm{~b}(\mathrm{i}) \cdot{ }^{*} \mathrm{Wq}\right)+\operatorname{sum}\left(\mathrm{g} 3212 \mathrm{a}(\mathrm{i}) .{ }^{*} \mathrm{Wq}\right)+\operatorname{sum}(\mathrm{g} 32\right.\right.$ $12 \mathrm{~b}(\mathrm{i}) . * \mathrm{Wq})))$

$\left(\mathrm{L}(4,9) *\left(\operatorname{sum}\left(\mathrm{g} 1233 \mathrm{a}(\mathrm{i}) .{ }^{*} \mathrm{Wq}\right)+\operatorname{sum}\left(\mathrm{g} 1233 \mathrm{~b}(\mathrm{i}) .{ }^{*} \mathrm{Wq}\right)+\operatorname{sum}\left(\mathrm{g} 3213 \mathrm{a}(\mathrm{i}) .{ }^{*} \mathrm{Wq}\right)+\operatorname{sum}(\mathrm{g} 32\right.\right.$ $13 \mathrm{~b}(\mathrm{i}) . * \mathrm{Wq})))$

$\left(\mathrm{L}(5,9) *\left(\operatorname{sum}\left(\mathrm{g} 1331 \mathrm{a}(\mathrm{i}) .{ }^{*} \mathrm{Wq}\right)+\operatorname{sum}\left(\mathrm{g} 1331 \mathrm{~b}(\mathrm{i}) \cdot{ }^{*} \mathrm{Wq}\right)+\operatorname{sum}\left(\mathrm{g} 3311 \mathrm{a}(\mathrm{i}) .{ }^{*} \mathrm{Wq}\right)+\operatorname{sum}(\mathrm{g} 33\right.\right.$ $11 \mathrm{~b}(\mathrm{i}) . * \mathrm{Wq})))$

$\left(\mathrm{L}(7,9) *\left(\operatorname{sum}\left(\mathrm{g} 1332 \mathrm{a}(\mathrm{i}) .{ }^{*} \mathrm{Wq}\right)+\operatorname{sum}\left(\mathrm{g} 1332 \mathrm{~b}(\mathrm{i}) \cdot{ }^{*} \mathrm{Wq}\right)+\operatorname{sum}\left(\mathrm{g} 3312 \mathrm{a}(\mathrm{i}) .{ }^{*} \mathrm{Wq}\right)+\operatorname{sum}(\mathrm{g} 33\right.\right.$ $12 \mathrm{~b}(\mathrm{i}) . * \mathrm{Wq}))$ )

$\left(\mathrm{L}(3,9) *\left(\operatorname{sum}\left(\mathrm{g} 1333 \mathrm{a}(\mathrm{i}) .{ }^{*} \mathrm{Wq}\right)+\operatorname{sum}\left(\mathrm{g} 1333 \mathrm{~b}(\mathrm{i}) \cdot{ }^{*} \mathrm{Wq}\right)+\operatorname{sum}\left(\mathrm{g} 3313 \mathrm{a}(\mathrm{i}) \cdot{ }^{*} \mathrm{Wq}\right)+\operatorname{sum}(\mathrm{g} 33\right.\right.$ $\left.\left.\left.\left.13 \mathrm{~b}(\mathrm{i}) *{ }^{*} \mathrm{Wq}\right)\right)\right)\right) /(8 * \mathrm{pi})$;

S2111

$\left(\left(\mathrm{L}(1,1) *\left(\operatorname{sum}\left(\mathrm{g} 2111 \mathrm{a}(\mathrm{i}) .{ }^{*} \mathrm{Wq}\right)+\operatorname{sum}\left(\mathrm{g} 2111 \mathrm{~b}(\mathrm{i}){ }^{*} \mathrm{Wq}\right)+\operatorname{sum}\left(\mathrm{g} 1121 \mathrm{a}(\mathrm{i}) .{ }^{*} \mathrm{Wq}\right)+\operatorname{sum}(\mathrm{g} 1\right.\right.\right.$ $121 \mathrm{~b}(\mathrm{i}) \cdot * \mathrm{Wq})))$

$\left(\mathrm{L}(6,1) *\left(\operatorname{sum}\left(\mathrm{g} 2112 \mathrm{a}(\mathrm{i}) .{ }^{*} \mathrm{Wq}\right)+\operatorname{sum}\left(\mathrm{g} 2112 \mathrm{~b}(\mathrm{i}) .{ }^{*} \mathrm{Wq}\right)+\operatorname{sum}\left(\mathrm{g} 1122 \mathrm{a}(\mathrm{i}) .{ }^{*} \mathrm{Wq}\right)+\operatorname{sum}(\mathrm{g} 11\right.\right.$ $22 \mathrm{~b}(\mathrm{i}) . * \mathrm{Wq})))$

$\left(\mathrm{L}(8,1) *\left(\operatorname{sum}\left(\mathrm{g} 2113 \mathrm{a}(\mathrm{i}) .{ }^{*} \mathrm{Wq}\right)+\operatorname{sum}\left(\mathrm{g} 2113 \mathrm{~b}(\mathrm{i}) .{ }^{*} \mathrm{Wq}\right)+\operatorname{sum}\left(\mathrm{g} 1123 \mathrm{a}(\mathrm{i}) .{ }^{*} \mathrm{Wq}\right)+\operatorname{sum}(\mathrm{g} 11\right.\right.$ $23 \mathrm{~b}(\mathrm{i}) . * \mathrm{Wq})))$

$\left(\mathrm{L}(9,1) *\left(\operatorname{sum}\left(\mathrm{g} 2211 \mathrm{a}(\mathrm{i}) .{ }^{*} \mathrm{Wq}\right)+\operatorname{sum}\left(\mathrm{g} 2211 \mathrm{~b}(\mathrm{i}) .{ }^{*} \mathrm{Wq}\right)+\operatorname{sum}\left(\mathrm{g} 1221 \mathrm{a}(\mathrm{i}) .{ }^{*} \mathrm{Wq}\right)+\operatorname{sum}(\mathrm{g} 12\right.\right.$ $21 \mathrm{~b}(\mathrm{i}) . * \mathrm{Wq})))$

$\left(\mathrm{L}(2,1) *\left(\operatorname{sum}\left(\mathrm{g} 2212 \mathrm{a}(\mathrm{i}) .{ }^{*} \mathrm{Wq}\right)+\operatorname{sum}\left(\mathrm{g} 2212 \mathrm{~b}(\mathrm{i}) \cdot{ }^{*} \mathrm{Wq}\right)+\operatorname{sum}\left(\mathrm{g} 1222 \mathrm{a}(\mathrm{i}) .{ }^{*} \mathrm{Wq}\right)+\operatorname{sum}(\mathrm{g} 12\right.\right.$ $22 \mathrm{~b}(\mathrm{i}) . * \mathrm{Wq})))$

$\left(\mathrm{L}(4,1) *\left(\operatorname{sum}\left(\mathrm{g} 2213 \mathrm{a}(\mathrm{i}) .{ }^{*} \mathrm{Wq}\right)+\operatorname{sum}\left(\mathrm{g} 2213 \mathrm{~b}(\mathrm{i}) .{ }^{*} \mathrm{Wq}\right)+\operatorname{sum}\left(\mathrm{g} 1223 \mathrm{a}(\mathrm{i}) .{ }^{*} \mathrm{Wq}\right)+\operatorname{sum}(\mathrm{g} 12\right.\right.$ 23b(i).*Wq)))

$\left(\mathrm{L}(5,1) *\left(\operatorname{sum}\left(\mathrm{g} 2311 \mathrm{a}(\mathrm{i}) .{ }^{*} \mathrm{Wq}\right)+\operatorname{sum}\left(\mathrm{g} 2311 \mathrm{~b}(\mathrm{i}) .{ }^{*} \mathrm{Wq}\right)+\operatorname{sum}\left(\mathrm{g} 1321 \mathrm{a}(\mathrm{i}) .{ }^{*} \mathrm{Wq}\right)+\operatorname{sum}(\mathrm{g} 13\right.\right.$ $\left.\left.\left.21 \mathrm{~b}(\mathrm{i}) *{ }^{*} \mathrm{Wq}\right)\right)\right)$

$\left(\mathrm{L}(7,1) *\left(\operatorname{sum}\left(\mathrm{g} 2312 \mathrm{a}(\mathrm{i}) .{ }^{*} \mathrm{Wq}\right)+\operatorname{sum}(\mathrm{g} 2312 \mathrm{~b}(\mathrm{i}) . * \mathrm{Wq})+\operatorname{sum}\left(\mathrm{g} 1322 \mathrm{a}(\mathrm{i}) .{ }^{*} \mathrm{Wq}\right)+\operatorname{sum}(\mathrm{g} 13\right.\right.$ $\left.\left.\left.22 \mathrm{~b}(\mathrm{i}) *{ }^{*} \mathrm{Wq}\right)\right)\right)$

$\left(\mathrm{L}(3,1) *\left(\operatorname{sum}\left(\mathrm{g} 2313 \mathrm{a}(\mathrm{i}) .{ }^{*} \mathrm{Wq}\right)+\operatorname{sum}\left(\mathrm{g} 2313 \mathrm{~b}(\mathrm{i}) \cdot{ }^{*} \mathrm{Wq}\right)+\operatorname{sum}\left(\mathrm{g} 1323 \mathrm{a}(\mathrm{i}) .{ }^{*} \mathrm{Wq}\right)+\operatorname{sum}(\mathrm{g} 13\right.\right.$ $23 \mathrm{~b}(\mathrm{i}) * \mathrm{Wq})))) /(8 * \mathrm{pi})$;

$\mathrm{S} 2122$

$\left(\left(\mathrm{L}(1,2) *\left(\operatorname{sum}\left(\mathrm{g} 2111 \mathrm{a}(\mathrm{i}) .{ }^{*} \mathrm{Wq}\right)+\operatorname{sum}\left(\mathrm{g} 2111 \mathrm{~b}(\mathrm{i}) .{ }^{*} \mathrm{Wq}\right)+\operatorname{sum}\left(\mathrm{g} 1121 \mathrm{a}(\mathrm{i}) .{ }^{*} \mathrm{Wq}\right)+\operatorname{sum}(\mathrm{g} 1\right.\right.\right.$ $\left.\left.\left.121 \mathrm{~b}(\mathrm{i}) \cdot{ }^{*} \mathrm{Wq}\right)\right)\right)$

$\left(\mathrm{L}(6,2) *\left(\operatorname{sum}\left(\mathrm{g} 2112 \mathrm{a}(\mathrm{i}) \cdot{ }^{*} \mathrm{Wq}\right)+\operatorname{sum}\left(\mathrm{g} 2112 \mathrm{~b}(\mathrm{i}) \cdot{ }^{*} \mathrm{Wq}\right)+\operatorname{sum}\left(\mathrm{g} 1122 \mathrm{a}(\mathrm{i}) \cdot{ }^{*} \mathrm{Wq}\right)+\operatorname{sum}(\mathrm{g} 11\right.\right.$ 
$22 \mathrm{~b}(\mathrm{i}) \cdot * \mathrm{Wq})))$

$\left(\mathrm{L}(8,2) *\left(\operatorname{sum}\left(\mathrm{g} 2113 \mathrm{a}(\mathrm{i}) .{ }^{*} \mathrm{Wq}\right)+\operatorname{sum}\left(\mathrm{g} 2113 \mathrm{~b}(\mathrm{i}) .{ }^{*} \mathrm{Wq}\right)+\operatorname{sum}\left(\mathrm{g} 1123 \mathrm{a}(\mathrm{i}) .{ }^{*} \mathrm{Wq}\right)+\operatorname{sum}(\mathrm{g} 11\right.\right.$

$23 \mathrm{~b}(\mathrm{i}) . * \mathrm{Wq})))$

$\left(\mathrm{L}(9,2) *\left(\operatorname{sum}\left(\mathrm{g} 2211 \mathrm{a}(\mathrm{i}) .{ }^{*} \mathrm{Wq}\right)+\operatorname{sum}\left(\mathrm{g} 2211 \mathrm{~b}(\mathrm{i}) .{ }^{*} \mathrm{Wq}\right)+\operatorname{sum}\left(\mathrm{g} 1221 \mathrm{a}(\mathrm{i}) .{ }^{*} \mathrm{Wq}\right)+\operatorname{sum}(\mathrm{g} 12\right.\right.$

$21 \mathrm{~b}(\mathrm{i}) \cdot * \mathrm{Wq})))$

$\left(\mathrm{L}(2,2) *\left(\operatorname{sum}\left(\mathrm{g} 2212 \mathrm{a}(\mathrm{i}) \cdot{ }^{*} \mathrm{Wq}\right)+\operatorname{sum}\left(\mathrm{g} 2212 \mathrm{~b}(\mathrm{i}) \cdot{ }^{*} \mathrm{Wq}\right)+\operatorname{sum}\left(\mathrm{g} 1222 \mathrm{a}(\mathrm{i}) .{ }^{*} \mathrm{Wq}\right)+\operatorname{sum}(\mathrm{g} 12\right.\right.$

$22 \mathrm{~b}(\mathrm{i}) . * \mathrm{Wq})))$

$\left(\mathrm{L}(4,2) *\left(\operatorname{sum}\left(\mathrm{g} 2213 \mathrm{a}(\mathrm{i}) .{ }^{*} \mathrm{Wq}\right)+\operatorname{sum}(\mathrm{g} 2213 \mathrm{~b}(\mathrm{i}) . * \mathrm{Wq})+\operatorname{sum}\left(\mathrm{g} 1223 \mathrm{a}(\mathrm{i}) .{ }^{*} \mathrm{Wq}\right)+\operatorname{sum}(\mathrm{g} 12\right.\right.$ 23b(i).*Wq))

$\left(\mathrm{L}(5,2) *\left(\operatorname{sum}\left(\mathrm{g} 2311 \mathrm{a}(\mathrm{i}) .{ }^{*} \mathrm{Wq}\right)+\operatorname{sum}\left(\mathrm{g} 2311 \mathrm{~b}(\mathrm{i}) .{ }^{*} \mathrm{Wq}\right)+\operatorname{sum}\left(\mathrm{g} 1321 \mathrm{a}(\mathrm{i}) .{ }^{*} \mathrm{Wq}\right)+\operatorname{sum}(\mathrm{g} 13\right.\right.$

$\left.\left.\left.21 \mathrm{~b}(\mathrm{i}) *{ }^{*} \mathrm{Wq}\right)\right)\right)$

$\left(\mathrm{L}(7,2) *\left(\operatorname{sum}\left(\mathrm{g} 2312 \mathrm{a}(\mathrm{i}) .{ }^{*} \mathrm{Wq}\right)+\operatorname{sum}\left(\mathrm{g} 2312 \mathrm{~b}(\mathrm{i}) .{ }^{*} \mathrm{Wq}\right)+\operatorname{sum}\left(\mathrm{g} 1322 \mathrm{a}(\mathrm{i}) .{ }^{*} \mathrm{Wq}\right)+\operatorname{sum}(\mathrm{g} 13\right.\right.$ $22 \mathrm{~b}(\mathrm{i}) . * \mathrm{Wq})))$

$\left(\mathrm{L}(3,2) *\left(\operatorname{sum}\left(\mathrm{g} 2313 \mathrm{a}(\mathrm{i}) .{ }^{*} \mathrm{Wq}\right)+\operatorname{sum}\left(\mathrm{g} 2313 \mathrm{~b}(\mathrm{i}) .{ }^{*} \mathrm{Wq}\right)+\operatorname{sum}\left(\mathrm{g} 1323 \mathrm{a}(\mathrm{i}) .{ }^{*} \mathrm{Wq}\right)+\operatorname{sum}(\mathrm{g} 13\right.\right.$ $23 \mathrm{~b}(\mathrm{i}) * \mathrm{Wq})))) /\left(8^{*} \mathrm{pi}\right)$;

$\mathrm{S} 2133$

$((\mathrm{L}(1,3) *(\operatorname{sum}(\mathrm{g} 2111 \mathrm{a}(\mathrm{i}) . * \mathrm{Wq})+\operatorname{sum}(\mathrm{g} 2111 \mathrm{~b}(\mathrm{i}) . * \mathrm{Wq})+\operatorname{sum}(\mathrm{g} 1121 \mathrm{a}(\mathrm{i}) . * \mathrm{Wq})+\operatorname{sum}(\mathrm{g} 1$ $121 \mathrm{~b}(\mathrm{i}) * * \mathrm{Wq})))$

$\left(\mathrm{L}(6,3) *\left(\operatorname{sum}\left(\mathrm{g} 2112 \mathrm{a}(\mathrm{i}) .{ }^{*} \mathrm{Wq}\right)+\operatorname{sum}\left(\mathrm{g} 2112 \mathrm{~b}(\mathrm{i}) .{ }^{*} \mathrm{Wq}\right)+\operatorname{sum}\left(\mathrm{g} 1122 \mathrm{a}(\mathrm{i}) .{ }^{*} \mathrm{Wq}\right)+\operatorname{sum}(\mathrm{g} 11\right.\right.$ $22 \mathrm{~b}(\mathrm{i}) . * \mathrm{Wq})))$

$\left(\mathrm{L}(8,3) *\left(\operatorname{sum}\left(\mathrm{g} 2113 \mathrm{a}(\mathrm{i}) .{ }^{*} \mathrm{Wq}\right)+\operatorname{sum}\left(\mathrm{g} 2113 \mathrm{~b}(\mathrm{i}) \cdot{ }^{*} \mathrm{Wq}\right)+\operatorname{sum}\left(\mathrm{g} 1123 \mathrm{a}(\mathrm{i}) .{ }^{*} \mathrm{Wq}\right)+\operatorname{sum}(\mathrm{g} 11\right.\right.$

$23 \mathrm{~b}(\mathrm{i}) . * \mathrm{Wq})))$

$\left(\mathrm{L}(9,3) *\left(\operatorname{sum}\left(\mathrm{g} 2211 \mathrm{a}(\mathrm{i}) .{ }^{*} \mathrm{Wq}\right)+\operatorname{sum}\left(\mathrm{g} 2211 \mathrm{~b}(\mathrm{i}) .{ }^{*} \mathrm{Wq}\right)+\operatorname{sum}\left(\mathrm{g} 1221 \mathrm{a}(\mathrm{i}) .{ }^{*} \mathrm{Wq}\right)+\operatorname{sum}(\mathrm{g} 12\right.\right.$

$21 \mathrm{~b}(\mathrm{i}) . * \mathrm{Wq})))$

$\left(\mathrm{L}(2,3) *\left(\operatorname{sum}\left(\mathrm{g} 2212 \mathrm{a}(\mathrm{i}) \cdot{ }^{*} \mathrm{Wq}\right)+\operatorname{sum}\left(\mathrm{g} 2212 \mathrm{~b}(\mathrm{i}) \cdot{ }^{*} \mathrm{Wq}\right)+\operatorname{sum}\left(\mathrm{g} 1222 \mathrm{a}(\mathrm{i}) \cdot{ }^{*} \mathrm{Wq}\right)+\operatorname{sum}(\mathrm{g} 12\right.\right.$

$22 \mathrm{~b}(\mathrm{i}) . * \mathrm{Wq})))$

$\left(\mathrm{L}(4,3) *\left(\operatorname{sum}\left(\mathrm{g} 2213 \mathrm{a}(\mathrm{i}) .{ }^{*} \mathrm{Wq}\right)+\operatorname{sum}\left(\mathrm{g} 2213 \mathrm{~b}(\mathrm{i}) .{ }^{*} \mathrm{Wq}\right)+\operatorname{sum}\left(\mathrm{g} 1223 \mathrm{a}(\mathrm{i}) \cdot{ }^{*} \mathrm{Wq}\right)+\operatorname{sum}(\mathrm{g} 12\right.\right.$ $23 \mathrm{~b}(\mathrm{i}) . * \mathrm{Wq}))$

$\left(\mathrm{L}(5,3) *\left(\operatorname{sum}\left(\mathrm{g} 2311 \mathrm{a}(\mathrm{i}) .{ }^{*} \mathrm{Wq}\right)+\operatorname{sum}\left(\mathrm{g} 2311 \mathrm{~b}(\mathrm{i}) \cdot{ }^{*} \mathrm{Wq}\right)+\operatorname{sum}\left(\mathrm{g} 1321 \mathrm{a}(\mathrm{i}) .{ }^{*} \mathrm{Wq}\right)+\operatorname{sum}(\mathrm{g} 13\right.\right.$

$21 \mathrm{~b}(\mathrm{i}) . * \mathrm{Wq})))$

$\left(\mathrm{L}(7,3) *\left(\operatorname{sum}\left(\mathrm{g} 2312 \mathrm{a}(\mathrm{i}) .{ }^{*} \mathrm{Wq}\right)+\operatorname{sum}\left(\mathrm{g} 2312 \mathrm{~b}(\mathrm{i}) .{ }^{*} \mathrm{Wq}\right)+\operatorname{sum}(\mathrm{g} 1322 \mathrm{a}(\mathrm{i}) . * \mathrm{Wq})+\operatorname{sum}(\mathrm{g} 13\right.\right.$

22b(i).*Wq))) +

$\left(\mathrm{L}(3,3) *\left(\operatorname{sum}\left(\mathrm{g} 2313 \mathrm{a}(\mathrm{i}) .{ }^{*} \mathrm{Wq}\right)+\operatorname{sum}\left(\mathrm{g} 2313 \mathrm{~b}(\mathrm{i}) \cdot{ }^{*} \mathrm{Wq}\right)+\operatorname{sum}\left(\mathrm{g} 1323 \mathrm{a}(\mathrm{i}) .{ }^{*} \mathrm{Wq}\right)+\operatorname{sum}(\mathrm{g} 13\right.\right.$

$\left.\left.\left.\left.23 \mathrm{~b}(\mathrm{i}) \cdot{ }^{*} \mathrm{Wq}\right)\right)\right)\right) /(8 * \mathrm{pi})$;

$\mathrm{S} 2123$

$\left(\left(\mathrm{L}(1,4) *\left(\operatorname{sum}\left(\mathrm{g} 2111 \mathrm{a}(\mathrm{i}) .{ }^{*} \mathrm{Wq}\right)+\operatorname{sum}\left(\mathrm{g} 2111 \mathrm{~b}(\mathrm{i}) .{ }^{*} \mathrm{Wq}\right)+\operatorname{sum}\left(\mathrm{g} 1121 \mathrm{a}(\mathrm{i}) .{ }^{*} \mathrm{Wq}\right)+\operatorname{sum}(\mathrm{g} 1\right.\right.\right.$ $\left.\left.\left.121 \mathrm{~b}(\mathrm{i}) *{ }^{*} \mathrm{Wq}\right)\right)\right)$

$\left(\mathrm{L}(6,4) *\left(\operatorname{sum}\left(\mathrm{g} 2112 \mathrm{a}(\mathrm{i}) .{ }^{*} \mathrm{Wq}\right)+\operatorname{sum}\left(\mathrm{g} 2112 \mathrm{~b}(\mathrm{i}) .{ }^{*} \mathrm{Wq}\right)+\operatorname{sum}\left(\mathrm{g} 1122 \mathrm{a}(\mathrm{i}) .{ }^{*} \mathrm{Wq}\right)+\operatorname{sum}(\mathrm{g} 11\right.\right.$ $\left.\left.\left.22 \mathrm{~b}(\mathrm{i}){ }^{*} \mathrm{Wq}\right)\right)\right)$

$\left(\mathrm{L}(8,4) *\left(\operatorname{sum}\left(\mathrm{g} 2113 \mathrm{a}(\mathrm{i}) .{ }^{*} \mathrm{Wq}\right)+\operatorname{sum}\left(\mathrm{g} 2113 \mathrm{~b}(\mathrm{i}) .{ }^{*} \mathrm{Wq}\right)+\operatorname{sum}\left(\mathrm{g} 1123 \mathrm{a}(\mathrm{i}) .{ }^{*} \mathrm{Wq}\right)+\operatorname{sum}(\mathrm{g} 11\right.\right.$

$23 \mathrm{~b}(\mathrm{i}) . * \mathrm{Wq})))$

$\left(\mathrm{L}(9,4) *\left(\operatorname{sum}\left(\mathrm{g} 2211 \mathrm{a}(\mathrm{i}) .{ }^{*} \mathrm{Wq}\right)+\operatorname{sum}\left(\mathrm{g} 2211 \mathrm{~b}(\mathrm{i}) .{ }^{*} \mathrm{Wq}\right)+\operatorname{sum}\left(\mathrm{g} 1221 \mathrm{a}(\mathrm{i}) .{ }^{*} \mathrm{Wq}\right)+\operatorname{sum}(\mathrm{g} 12\right.\right.$

$\left.\left.\left.21 \mathrm{~b}(\mathrm{i}) \cdot{ }^{*} \mathrm{Wq}\right)\right)\right)$ 
$\left(\mathrm{L}(2,4) *\left(\operatorname{sum}\left(\mathrm{g} 2212 \mathrm{a}(\mathrm{i}) .{ }^{*} \mathrm{Wq}\right)+\operatorname{sum}\left(\mathrm{g} 2212 \mathrm{~b}(\mathrm{i}) \cdot{ }^{*} \mathrm{Wq}\right)+\operatorname{sum}\left(\mathrm{g} 1222 \mathrm{a}(\mathrm{i}) .{ }^{*} \mathrm{Wq}\right)+\operatorname{sum}(\mathrm{g} 12\right.\right.$ $22 \mathrm{~b}(\mathrm{i}) . * \mathrm{Wq})))$

$\left(\mathrm{L}(4,4) *\left(\operatorname{sum}(\mathrm{g} 2213 \mathrm{a}(\mathrm{i}) . * \mathrm{Wq})+\operatorname{sum}\left(\mathrm{g} 2213 \mathrm{~b}(\mathrm{i}) \cdot{ }^{*} \mathrm{Wq}\right)+\operatorname{sum}\left(\mathrm{g} 1223 \mathrm{a}(\mathrm{i}) .{ }^{*} \mathrm{Wq}\right)+\operatorname{sum}(\mathrm{g} 12\right.\right.$ 23b(i).*Wq)))

$\left(\mathrm{L}(5,4) *\left(\operatorname{sum}\left(\mathrm{g} 2311 \mathrm{a}(\mathrm{i}) .{ }^{*} \mathrm{Wq}\right)+\operatorname{sum}\left(\mathrm{g} 2311 \mathrm{~b}(\mathrm{i}) .{ }^{*} \mathrm{Wq}\right)+\operatorname{sum}(\mathrm{g} 1321 \mathrm{a}(\mathrm{i}) . * \mathrm{Wq})+\operatorname{sum}(\mathrm{g} 13\right.\right.$ $21 \mathrm{~b}(\mathrm{i}) . * \mathrm{Wq})))$

$\left(\mathrm{L}(7,4) *\left(\operatorname{sum}\left(\mathrm{g} 2312 \mathrm{a}(\mathrm{i}) .{ }^{*} \mathrm{Wq}\right)+\operatorname{sum}\left(\mathrm{g} 2312 \mathrm{~b}(\mathrm{i}) \cdot{ }^{*} \mathrm{Wq}\right)+\operatorname{sum}\left(\mathrm{g} 1322 \mathrm{a}(\mathrm{i}) .{ }^{*} \mathrm{Wq}\right)+\operatorname{sum}(\mathrm{g} 13\right.\right.$ $22 \mathrm{~b}(\mathrm{i}) . * \mathrm{Wq})))$

$\left(\mathrm{L}(3,4) *\left(\operatorname{sum}\left(\mathrm{g} 2313 \mathrm{a}(\mathrm{i}) .{ }^{*} \mathrm{Wq}\right)+\operatorname{sum}\left(\mathrm{g} 2313 \mathrm{~b}(\mathrm{i}) .{ }^{*} \mathrm{Wq}\right)+\operatorname{sum}\left(\mathrm{g} 1323 \mathrm{a}(\mathrm{i}) .{ }^{*} \mathrm{Wq}\right)+\operatorname{sum}(\mathrm{g} 13\right.\right.$ $\left.\left.\left.\left.23 \mathrm{~b}(\mathrm{i}) *{ }^{*} \mathrm{Wq}\right)\right)\right)\right) /\left(8^{*} \mathrm{pi}\right)$;

$\mathrm{S} 2131$

$\left(\left(\mathrm{L}(1,5) *\left(\operatorname{sum}(\mathrm{g} 2111 \mathrm{a}(\mathrm{i}) . * \mathrm{Wq})+\operatorname{sum}\left(\mathrm{g} 2111 \mathrm{~b}(\mathrm{i}) .{ }^{*} \mathrm{Wq}\right)+\operatorname{sum}(\mathrm{g} 1121 \mathrm{a}(\mathrm{i}) . * \mathrm{Wq})+\operatorname{sum}(\mathrm{g} 1\right.\right.\right.$ $\left.\left.\left.121 \mathrm{~b}(\mathrm{i}) \cdot{ }^{*} \mathrm{Wq}\right)\right)\right)$

$\left(\mathrm{L}(6,5) *\left(\operatorname{sum}\left(\mathrm{g} 2112 \mathrm{a}(\mathrm{i}) .{ }^{*} \mathrm{Wq}\right)+\operatorname{sum}\left(\mathrm{g} 2112 \mathrm{~b}(\mathrm{i}) .{ }^{*} \mathrm{Wq}\right)+\operatorname{sum}\left(\mathrm{g} 1122 \mathrm{a}(\mathrm{i}) .{ }^{*} \mathrm{Wq}\right)+\operatorname{sum}(\mathrm{g} 11\right.\right.$ $22 \mathrm{~b}(\mathrm{i}) . * \mathrm{Wq}))$ )

$\left(\mathrm{L}(8,5) *\left(\operatorname{sum}\left(\mathrm{g} 2113 \mathrm{a}(\mathrm{i}) .{ }^{*} \mathrm{Wq}\right)+\operatorname{sum}\left(\mathrm{g} 2113 \mathrm{~b}(\mathrm{i}) .{ }^{*} \mathrm{Wq}\right)+\operatorname{sum}\left(\mathrm{g} 1123 \mathrm{a}(\mathrm{i}) .{ }^{*} \mathrm{Wq}\right)+\operatorname{sum}(\mathrm{g} 11\right.\right.$ $23 \mathrm{~b}(\mathrm{i}) . * \mathrm{Wq})))$

$\left(\mathrm{L}(9,5) *\left(\operatorname{sum}\left(\mathrm{g} 2211 \mathrm{a}(\mathrm{i}) \cdot{ }^{*} \mathrm{Wq}\right)+\operatorname{sum}\left(\mathrm{g} 2211 \mathrm{~b}(\mathrm{i}) .{ }^{*} \mathrm{Wq}\right)+\operatorname{sum}\left(\mathrm{g} 1221 \mathrm{a}(\mathrm{i}) \cdot{ }^{*} \mathrm{Wq}\right)+\operatorname{sum}(\mathrm{g} 12\right.\right.$ $21 \mathrm{~b}(\mathrm{i}) . * \mathrm{Wq}))$

$\left(\mathrm{L}(2,5) *\left(\operatorname{sum}\left(\mathrm{g} 2212 \mathrm{a}(\mathrm{i}) .{ }^{*} \mathrm{Wq}\right)+\operatorname{sum}\left(\mathrm{g} 2212 \mathrm{~b}(\mathrm{i}) \cdot{ }^{*} \mathrm{Wq}\right)+\operatorname{sum}\left(\mathrm{g} 1222 \mathrm{a}(\mathrm{i}) \cdot{ }^{*} \mathrm{Wq}\right)+\operatorname{sum}(\mathrm{g} 12\right.\right.$ $22 \mathrm{~b}(\mathrm{i}) . * \mathrm{Wq})))$

$\left(\mathrm{L}(4,5) *\left(\operatorname{sum}(\mathrm{g} 2213 \mathrm{a}(\mathrm{i}) . * \mathrm{Wq})+\operatorname{sum}\left(\mathrm{g} 2213 \mathrm{~b}(\mathrm{i}) \cdot{ }^{*} \mathrm{Wq}\right)+\operatorname{sum}\left(\mathrm{g} 1223 \mathrm{a}(\mathrm{i}) \cdot{ }^{*} \mathrm{Wq}\right)+\operatorname{sum}(\mathrm{g} 12\right.\right.$ $23 \mathrm{~b}(\mathrm{i}) . * \mathrm{Wq}))$

$\left(\mathrm{L}(5,5) *\left(\operatorname{sum}\left(\mathrm{g} 2311 \mathrm{a}(\mathrm{i}) .{ }^{*} \mathrm{Wq}\right)+\operatorname{sum}\left(\mathrm{g} 2311 \mathrm{~b}(\mathrm{i}) \cdot{ }^{*} \mathrm{Wq}\right)+\operatorname{sum}\left(\mathrm{g} 1321 \mathrm{a}(\mathrm{i}) .{ }^{*} \mathrm{Wq}\right)+\operatorname{sum}(\mathrm{g} 13\right.\right.$ $21 \mathrm{~b}(\mathrm{i}) . * \mathrm{Wq})))$

$\left(\mathrm{L}(7,5) *\left(\operatorname{sum}\left(\mathrm{g} 2312 \mathrm{a}(\mathrm{i}) .{ }^{*} \mathrm{Wq}\right)+\operatorname{sum}\left(\mathrm{g} 2312 \mathrm{~b}(\mathrm{i}) .{ }^{*} \mathrm{Wq}\right)+\operatorname{sum}\left(\mathrm{g} 1322 \mathrm{a}(\mathrm{i}) .{ }^{*} \mathrm{Wq}\right)+\operatorname{sum}(\mathrm{g} 13\right.\right.$ $22 \mathrm{~b}(\mathrm{i}) . * \mathrm{Wq})))$

$\left(\mathrm{L}(3,5) *\left(\operatorname{sum}\left(\mathrm{g} 2313 \mathrm{a}(\mathrm{i}) .{ }^{*} \mathrm{Wq}\right)+\operatorname{sum}\left(\mathrm{g} 2313 \mathrm{~b}(\mathrm{i}) .{ }^{*} \mathrm{Wq}\right)+\operatorname{sum}\left(\mathrm{g} 1323 \mathrm{a}(\mathrm{i}) .{ }^{*} \mathrm{Wq}\right)+\operatorname{sum}(\mathrm{g} 13\right.\right.$ $23 \mathrm{~b}(\mathrm{i}) . * \mathrm{Wq})))) /(8 * \mathrm{pi})$;

$\mathrm{S} 2112$

$\left(\left(\mathrm{L}(1,6) *\left(\operatorname{sum}\left(\mathrm{g} 2111 \mathrm{a}(\mathrm{i}) .{ }^{*} \mathrm{Wq}\right)+\operatorname{sum}\left(\mathrm{g} 2111 \mathrm{~b}(\mathrm{i}) .{ }^{*} \mathrm{Wq}\right)+\operatorname{sum}\left(\mathrm{g} 1121 \mathrm{a}(\mathrm{i}) .{ }^{*} \mathrm{Wq}\right)+\operatorname{sum}(\mathrm{g} 1\right.\right.\right.$ $121 \mathrm{~b}(\mathrm{i}) * \mathrm{Wq})))$

$\left(\mathrm{L}(6,6) *\left(\operatorname{sum}\left(\mathrm{g} 2112 \mathrm{a}(\mathrm{i}) .{ }^{*} \mathrm{Wq}\right)+\operatorname{sum}\left(\mathrm{g} 2112 \mathrm{~b}(\mathrm{i}) \cdot{ }^{*} \mathrm{Wq}\right)+\operatorname{sum}\left(\mathrm{g} 1122 \mathrm{a}(\mathrm{i}) .{ }^{*} \mathrm{Wq}\right)+\operatorname{sum}(\mathrm{g} 11\right.\right.$ $22 \mathrm{~b}(\mathrm{i}) . * \mathrm{Wq})))$

$\left(\mathrm{L}(8,6) *\left(\operatorname{sum}\left(\mathrm{g} 2113 \mathrm{a}(\mathrm{i}) .{ }^{*} \mathrm{Wq}\right)+\operatorname{sum}\left(\mathrm{g} 2113 \mathrm{~b}(\mathrm{i}) \cdot{ }^{*} \mathrm{Wq}\right)+\operatorname{sum}(\mathrm{g} 1123 \mathrm{a}(\mathrm{i}) . * \mathrm{Wq})+\operatorname{sum}(\mathrm{g} 11\right.\right.$ $23 \mathrm{~b}(\mathrm{i}) \cdot * \mathrm{Wq}))$

$\left(\mathrm{L}(9,6) *\left(\operatorname{sum}\left(\mathrm{g} 2211 \mathrm{a}(\mathrm{i}) .{ }^{*} \mathrm{Wq}\right)+\operatorname{sum}\left(\mathrm{g} 2211 \mathrm{~b}(\mathrm{i}) \cdot{ }^{*} \mathrm{Wq}\right)+\operatorname{sum}\left(\mathrm{g} 1221 \mathrm{a}(\mathrm{i}) \cdot{ }^{*} \mathrm{Wq}\right)+\operatorname{sum}(\mathrm{g} 12\right.\right.$ $21 \mathrm{~b}(\mathrm{i}) . * \mathrm{Wq})))$

$\left(\mathrm{L}(2,6) *\left(\operatorname{sum}\left(\mathrm{g} 2212 \mathrm{a}(\mathrm{i}) .{ }^{*} \mathrm{Wq}\right)+\operatorname{sum}\left(\mathrm{g} 2212 \mathrm{~b}(\mathrm{i}) \cdot{ }^{*} \mathrm{Wq}\right)+\operatorname{sum}\left(\mathrm{g} 1222 \mathrm{a}(\mathrm{i}) \cdot{ }^{*} \mathrm{Wq}\right)+\operatorname{sum}(\mathrm{g} 12\right.\right.$ $22 \mathrm{~b}(\mathrm{i}) . * \mathrm{Wq})))$

$\left(\mathrm{L}(4,6) *\left(\operatorname{sum}(\mathrm{g} 2213 \mathrm{a}(\mathrm{i}) . * \mathrm{Wq})+\operatorname{sum}\left(\mathrm{g} 2213 \mathrm{~b}(\mathrm{i}) .{ }^{*} \mathrm{Wq}\right)+\operatorname{sum}\left(\mathrm{g} 1223 \mathrm{a}(\mathrm{i}) .{ }^{*} \mathrm{Wq}\right)+\operatorname{sum}(\mathrm{g} 12\right.\right.$ $23 \mathrm{~b}(\mathrm{i}) . * \mathrm{Wq}))$

$\left(\mathrm{L}(5,6) *\left(\operatorname{sum}\left(\mathrm{g} 2311 \mathrm{a}(\mathrm{i}) .{ }^{*} \mathrm{Wq}\right)+\operatorname{sum}\left(\mathrm{g} 2311 \mathrm{~b}(\mathrm{i}) .{ }^{*} \mathrm{Wq}\right)+\operatorname{sum}\left(\mathrm{g} 1321 \mathrm{a}(\mathrm{i}) .{ }^{*} \mathrm{Wq}\right)+\operatorname{sum}(\mathrm{g} 13\right.\right.$ 
$21 \mathrm{~b}(\mathrm{i}) . * \mathrm{Wq})))$

$\left(\mathrm{L}(7,6) *\left(\operatorname{sum}\left(\mathrm{g} 2312 \mathrm{a}(\mathrm{i}) .{ }^{*} \mathrm{Wq}\right)+\operatorname{sum}\left(\mathrm{g} 2312 \mathrm{~b}(\mathrm{i}) .{ }^{*} \mathrm{Wq}\right)+\operatorname{sum}\left(\mathrm{g} 1322 \mathrm{a}(\mathrm{i}) .{ }^{*} \mathrm{Wq}\right)+\operatorname{sum}(\mathrm{g} 13\right.\right.$

$22 \mathrm{~b}(\mathrm{i}) . * \mathrm{Wq}))$ )

$\left(\mathrm{L}(3,6) *\left(\operatorname{sum}\left(\mathrm{g} 2313 \mathrm{a}(\mathrm{i}) .{ }^{*} \mathrm{Wq}\right)+\operatorname{sum}\left(\mathrm{g} 2313 \mathrm{~b}(\mathrm{i}) .{ }^{*} \mathrm{Wq}\right)+\operatorname{sum}\left(\mathrm{g} 1323 \mathrm{a}(\mathrm{i}) .{ }^{*} \mathrm{Wq}\right)+\operatorname{sum}(\mathrm{g} 13\right.\right.$

$\left.\left.\left.\left.23 \mathrm{~b}(\mathrm{i}) *{ }^{*} \mathrm{Wq}\right)\right)\right)\right) /(8 * \mathrm{pi})$;

$\mathrm{S} 2132$

$\left(\left(\mathrm{L}(1,7) *\left(\operatorname{sum}\left(\mathrm{g} 2111 \mathrm{a}(\mathrm{i}) .{ }^{*} \mathrm{Wq}\right)+\operatorname{sum}\left(\mathrm{g} 2111 \mathrm{~b}(\mathrm{i}) .{ }^{*} \mathrm{Wq}\right)+\operatorname{sum}\left(\mathrm{g} 1121 \mathrm{a}(\mathrm{i}) .{ }^{*} \mathrm{Wq}\right)+\operatorname{sum}(\mathrm{g} 1\right.\right.\right.$

$121 \mathrm{~b}(\mathrm{i}) \cdot * \mathrm{Wq})))$

$\left(\mathrm{L}(6,7) *\left(\operatorname{sum}\left(\mathrm{g} 2112 \mathrm{a}(\mathrm{i}) .{ }^{*} \mathrm{Wq}\right)+\operatorname{sum}\left(\mathrm{g} 2112 \mathrm{~b}(\mathrm{i}) .{ }^{*} \mathrm{Wq}\right)+\operatorname{sum}\left(\mathrm{g} 1122 \mathrm{a}(\mathrm{i}) .{ }^{*} \mathrm{Wq}\right)+\operatorname{sum}(\mathrm{g} 11\right.\right.$

$22 \mathrm{~b}(\mathrm{i}) . * \mathrm{Wq}))$ )

$\left(\mathrm{L}(8,7) *\left(\operatorname{sum}\left(\mathrm{g} 2113 \mathrm{a}(\mathrm{i}) .{ }^{*} \mathrm{Wq}\right)+\operatorname{sum}(\mathrm{g} 2113 \mathrm{~b}(\mathrm{i}) . * \mathrm{Wq})+\operatorname{sum}\left(\mathrm{g} 1123 \mathrm{a}(\mathrm{i}) .{ }^{*} \mathrm{Wq}\right)+\operatorname{sum}(\mathrm{g} 11\right.\right.$

23b(i).*Wq)))

$\left(\mathrm{L}(9,7) *\left(\operatorname{sum}\left(\mathrm{g} 2211 \mathrm{a}(\mathrm{i}) .{ }^{*} \mathrm{Wq}\right)+\operatorname{sum}\left(\mathrm{g} 2211 \mathrm{~b}(\mathrm{i}) \cdot{ }^{*} \mathrm{Wq}\right)+\operatorname{sum}\left(\mathrm{g} 1221 \mathrm{a}(\mathrm{i}) .{ }^{*} \mathrm{Wq}\right)+\operatorname{sum}(\mathrm{g} 12\right.\right.$

$21 \mathrm{~b}(\mathrm{i}) . * \mathrm{Wq}))) \quad+$

$\left(\mathrm{L}(2,7) *\left(\operatorname{sum}\left(\mathrm{g} 2212 \mathrm{a}(\mathrm{i}) .{ }^{*} \mathrm{Wq}\right)+\operatorname{sum}\left(\mathrm{g} 2212 \mathrm{~b}(\mathrm{i}) \cdot{ }^{*} \mathrm{Wq}\right)+\operatorname{sum}\left(\mathrm{g} 1222 \mathrm{a}(\mathrm{i}) .{ }^{*} \mathrm{Wq}\right)+\operatorname{sum}(\mathrm{g} 12\right.\right.$

$\left.\left.\left.22 \mathrm{~b}(\mathrm{i}) *{ }^{*} \mathrm{Wq}\right)\right)\right)$

$\left(\mathrm{L}(4,7) *\left(\operatorname{sum}(\mathrm{g} 2213 \mathrm{a}(\mathrm{i}) . * \mathrm{Wq})+\operatorname{sum}\left(\mathrm{g} 2213 \mathrm{~b}(\mathrm{i}) \cdot{ }^{*} \mathrm{Wq}\right)+\operatorname{sum}\left(\mathrm{g} 1223 \mathrm{a}(\mathrm{i}) .{ }^{*} \mathrm{Wq}\right)+\operatorname{sum}(\mathrm{g} 12\right.\right.$ 23b(i).*Wq))) +

$\left(\mathrm{L}(5,7) *\left(\operatorname{sum}\left(\mathrm{g} 2311 \mathrm{a}(\mathrm{i}) .{ }^{*} \mathrm{Wq}\right)+\operatorname{sum}\left(\mathrm{g} 2311 \mathrm{~b}(\mathrm{i}) .{ }^{*} \mathrm{Wq}\right)+\operatorname{sum}\left(\mathrm{g} 1321 \mathrm{a}(\mathrm{i}) .{ }^{*} \mathrm{Wq}\right)+\operatorname{sum}(\mathrm{g} 13\right.\right.$

$\left.\left.21 \mathrm{~b}(\mathrm{i}) \cdot{ }^{*} \mathrm{Wq}\right)\right)$ ) +

$\left(\mathrm{L}(7,7) *\left(\operatorname{sum}(\mathrm{g} 2312 \mathrm{a}(\mathrm{i}) . * \mathrm{Wq})+\operatorname{sum}\left(\mathrm{g} 2312 \mathrm{~b}(\mathrm{i}) .{ }^{*} \mathrm{Wq}\right)+\operatorname{sum}(\mathrm{g} 1322 \mathrm{a}(\mathrm{i}) . * \mathrm{Wq})+\operatorname{sum}(\mathrm{g} 13\right.\right.$ $22 \mathrm{~b}(\mathrm{i}) * \mathrm{Wq})))$

$\left(\mathrm{L}(3,7) *\left(\operatorname{sum}\left(\mathrm{g} 2313 \mathrm{a}(\mathrm{i}) .{ }^{*} \mathrm{Wq}\right)+\operatorname{sum}\left(\mathrm{g} 2313 \mathrm{~b}(\mathrm{i}) \cdot{ }^{*} \mathrm{Wq}\right)+\operatorname{sum}\left(\mathrm{g} 1323 \mathrm{a}(\mathrm{i}) .{ }^{*} \mathrm{Wq}\right)+\operatorname{sum}(\mathrm{g} 13\right.\right.$ $23 \mathrm{~b}(\mathrm{i}) *$ (Wq) $))) /(8 * \mathrm{pi})$;

$\mathrm{S} 2113$

$\left(\left(\mathrm{L}(1,8) *\left(\operatorname{sum}\left(\mathrm{g} 2111 \mathrm{a}(\mathrm{i}) .{ }^{*} \mathrm{Wq}\right)+\operatorname{sum}\left(\mathrm{g} 2111 \mathrm{~b}(\mathrm{i}) \cdot{ }^{*} \mathrm{Wq}\right)+\operatorname{sum}\left(\mathrm{g} 1121 \mathrm{a}(\mathrm{i}) .{ }^{*} \mathrm{Wq}\right)+\operatorname{sum}(\mathrm{g} 1\right.\right.\right.$ $\left.\left.\left.121 \mathrm{~b}(\mathrm{i}) *{ }^{*} \mathrm{Wq}\right)\right)\right)$

$\left(\mathrm{L}(6,8) *\left(\operatorname{sum}(\mathrm{g} 2112 \mathrm{a}(\mathrm{i}) . * \mathrm{Wq})+\operatorname{sum}\left(\mathrm{g} 2112 \mathrm{~b}(\mathrm{i}) .{ }^{*} \mathrm{Wq}\right)+\operatorname{sum}(\mathrm{g} 1122 \mathrm{a}(\mathrm{i}) . * \mathrm{Wq})+\operatorname{sum}(\mathrm{g} 11\right.\right.$ $22 \mathrm{~b}(\mathrm{i}) . * \mathrm{Wq}))$ )

$\left(\mathrm{L}(8,8) *\left(\operatorname{sum}\left(\mathrm{g} 2113 \mathrm{a}(\mathrm{i}) .{ }^{*} \mathrm{Wq}\right)+\operatorname{sum}\left(\mathrm{g} 2113 \mathrm{~b}(\mathrm{i}) .{ }^{*} \mathrm{Wq}\right)+\operatorname{sum}\left(\mathrm{g} 1123 \mathrm{a}(\mathrm{i}) .{ }^{*} \mathrm{Wq}\right)+\operatorname{sum}(\mathrm{g} 11\right.\right.$ $23 \mathrm{~b}(\mathrm{i}) . * \mathrm{Wq}))$ )

$\left(\mathrm{L}(9,8) *\left(\operatorname{sum}\left(\mathrm{g} 2211 \mathrm{a}(\mathrm{i}) .{ }^{*} \mathrm{Wq}\right)+\operatorname{sum}\left(\mathrm{g} 2211 \mathrm{~b}(\mathrm{i}) \cdot{ }^{*} \mathrm{Wq}\right)+\operatorname{sum}\left(\mathrm{g} 1221 \mathrm{a}(\mathrm{i}) .{ }^{*} \mathrm{Wq}\right)+\operatorname{sum}(\mathrm{g} 12\right.\right.$ $21 \mathrm{~b}(\mathrm{i}) . * \mathrm{Wq}))$ )

$\left(\mathrm{L}(2,8) *\left(\operatorname{sum}\left(\mathrm{g} 2212 \mathrm{a}(\mathrm{i}) \cdot{ }^{*} \mathrm{Wq}\right)+\operatorname{sum}\left(\mathrm{g} 2212 \mathrm{~b}(\mathrm{i}) \cdot{ }^{*} \mathrm{Wq}\right)+\operatorname{sum}\left(\mathrm{g} 1222 \mathrm{a}(\mathrm{i}) .{ }^{*} \mathrm{Wq}\right)+\operatorname{sum}(\mathrm{g} 12\right.\right.$ $22 \mathrm{~b}(\mathrm{i}) . * \mathrm{Wq})))$

$\left(\mathrm{L}(4,8) *\left(\operatorname{sum}\left(\mathrm{g} 2213 \mathrm{a}(\mathrm{i}) .{ }^{*} \mathrm{Wq}\right)+\operatorname{sum}\left(\mathrm{g} 2213 \mathrm{~b}(\mathrm{i}) .{ }^{*} \mathrm{Wq}\right)+\operatorname{sum}\left(\mathrm{g} 1223 \mathrm{a}(\mathrm{i}) .{ }^{*} \mathrm{Wq}\right)+\operatorname{sum}(\mathrm{g} 12\right.\right.$ 23b(i).*Wq))) + $\left(\mathrm{L}(5,8) *\left(\operatorname{sum}\left(\mathrm{g} 2311 \mathrm{a}(\mathrm{i}) .{ }^{*} \mathrm{Wq}\right)+\operatorname{sum}\left(\mathrm{g} 2311 \mathrm{~b}(\mathrm{i}) \cdot{ }^{*} \mathrm{Wq}\right)+\operatorname{sum}\left(\mathrm{g} 1321 \mathrm{a}(\mathrm{i}) .{ }^{*} \mathrm{Wq}\right)+\operatorname{sum}(\mathrm{g} 13\right.\right.$ $\left.\left.\left.21 \mathrm{~b}(\mathrm{i}) \cdot{ }^{*} \mathrm{Wq}\right)\right)\right)$ + $\left(\mathrm{L}(7,8) *\left(\operatorname{sum}\left(\mathrm{g} 2312 \mathrm{a}(\mathrm{i}) \cdot{ }^{*} \mathrm{Wq}\right)+\operatorname{sum}\left(\mathrm{g} 2312 \mathrm{~b}(\mathrm{i}) \cdot{ }^{*} \mathrm{Wq}\right)+\operatorname{sum}(\mathrm{g} 1322 \mathrm{a}(\mathrm{i}) . * \mathrm{Wq})+\operatorname{sum}(\mathrm{g} 13\right.\right.$ $22 \mathrm{~b}(\mathrm{i}) \cdot * \mathrm{Wq})))$ $\left(\mathrm{L}(3,8) *\left(\operatorname{sum}\left(\mathrm{g} 2313 \mathrm{a}(\mathrm{i}) .{ }^{*} \mathrm{Wq}\right)+\operatorname{sum}\left(\mathrm{g} 2313 \mathrm{~b}(\mathrm{i}) .{ }^{*} \mathrm{Wq}\right)+\operatorname{sum}\left(\mathrm{g} 1323 \mathrm{a}(\mathrm{i}) .{ }^{*} \mathrm{Wq}\right)+\operatorname{sum}(\mathrm{g} 13\right.\right.$ $23 \mathrm{~b}(\mathrm{i}) . * \mathrm{Wq})))) /(8 * \mathrm{pi})$; 
S2121

$\left(\left(\mathrm{L}(1,9) *\left(\operatorname{sum}\left(\mathrm{g} 2111 \mathrm{a}(\mathrm{i}) .{ }^{*} \mathrm{Wq}\right)+\operatorname{sum}\left(\mathrm{g} 2111 \mathrm{~b}(\mathrm{i}) \cdot{ }^{*} \mathrm{Wq}\right)+\operatorname{sum}\left(\mathrm{g} 1121 \mathrm{a}(\mathrm{i}) .{ }^{*} \mathrm{Wq}\right)+\operatorname{sum}(\mathrm{g} 1\right.\right.\right.$ $121 \mathrm{~b}(\mathrm{i}) \cdot * \mathrm{Wq})))$

$\left(\mathrm{L}(6,9) *\left(\operatorname{sum}\left(\mathrm{g} 2112 \mathrm{a}(\mathrm{i}) .{ }^{*} \mathrm{Wq}\right)+\operatorname{sum}\left(\mathrm{g} 2112 \mathrm{~b}(\mathrm{i}) \cdot{ }^{*} \mathrm{Wq}\right)+\operatorname{sum}\left(\mathrm{g} 1122 \mathrm{a}(\mathrm{i}) .{ }^{*} \mathrm{Wq}\right)+\operatorname{sum}(\mathrm{g} 11\right.\right.$ $\left.\left.\left.22 \mathrm{~b}(\mathrm{i}) \cdot{ }^{*} \mathrm{Wq}\right)\right)\right)$

$\left(\mathrm{L}(8,9) *\left(\operatorname{sum}\left(\mathrm{g} 2113 \mathrm{a}(\mathrm{i}) .{ }^{*} \mathrm{Wq}\right)+\operatorname{sum}\left(\mathrm{g} 2113 \mathrm{~b}(\mathrm{i}) .{ }^{*} \mathrm{Wq}\right)+\operatorname{sum}\left(\mathrm{g} 1123 \mathrm{a}(\mathrm{i}) .{ }^{*} \mathrm{Wq}\right)+\operatorname{sum}(\mathrm{g} 11\right.\right.$ $23 \mathrm{~b}(\mathrm{i}) . * \mathrm{Wq})))$

$\left(\mathrm{L}(9,9) *\left(\operatorname{sum}\left(\mathrm{g} 2211 \mathrm{a}(\mathrm{i}) .{ }^{*} \mathrm{Wq}\right)+\operatorname{sum}\left(\mathrm{g} 2211 \mathrm{~b}(\mathrm{i}) .{ }^{*} \mathrm{Wq}\right)+\operatorname{sum}\left(\mathrm{g} 1221 \mathrm{a}(\mathrm{i}) .{ }^{*} \mathrm{Wq}\right)+\operatorname{sum}(\mathrm{g} 12\right.\right.$ $21 \mathrm{~b}(\mathrm{i}) . * \mathrm{Wq})))$

$\left(\mathrm{L}(2,9) *\left(\operatorname{sum}\left(\mathrm{g} 2212 \mathrm{a}(\mathrm{i}) .{ }^{*} \mathrm{Wq}\right)+\operatorname{sum}\left(\mathrm{g} 2212 \mathrm{~b}(\mathrm{i}) .{ }^{*} \mathrm{Wq}\right)+\operatorname{sum}\left(\mathrm{g} 1222 \mathrm{a}(\mathrm{i}) .{ }^{*} \mathrm{Wq}\right)+\operatorname{sum}(\mathrm{g} 12\right.\right.$ $\left.\left.\left.22 \mathrm{~b}(\mathrm{i}) *{ }^{*} \mathrm{Wq}\right)\right)\right)$

$\left(\mathrm{L}(4,9) *\left(\operatorname{sum}\left(\mathrm{g} 2213 \mathrm{a}(\mathrm{i}) .{ }^{*} \mathrm{Wq}\right)+\operatorname{sum}\left(\mathrm{g} 2213 \mathrm{~b}(\mathrm{i}) .{ }^{*} \mathrm{Wq}\right)+\operatorname{sum}\left(\mathrm{g} 1223 \mathrm{a}(\mathrm{i}) .{ }^{*} \mathrm{Wq}\right)+\operatorname{sum}(\mathrm{g} 12\right.\right.$ 23b(i).*Wq)))

$\left(\mathrm{L}(5,9) *\left(\operatorname{sum}\left(\mathrm{g} 2311 \mathrm{a}(\mathrm{i}) .{ }^{*} \mathrm{Wq}\right)+\operatorname{sum}\left(\mathrm{g} 2311 \mathrm{~b}(\mathrm{i}) \cdot{ }^{*} \mathrm{Wq}\right)+\operatorname{sum}\left(\mathrm{g} 1321 \mathrm{a}(\mathrm{i}) .{ }^{*} \mathrm{Wq}\right)+\operatorname{sum}(\mathrm{g} 13\right.\right.$ $21 \mathrm{~b}(\mathrm{i}) . * \mathrm{Wq})))$

$\left(\mathrm{L}(7,9) *\left(\operatorname{sum}\left(\mathrm{g} 2312 \mathrm{a}(\mathrm{i}) .{ }^{*} \mathrm{Wq}\right)+\operatorname{sum}\left(\mathrm{g} 2312 \mathrm{~b}(\mathrm{i}) .{ }^{*} \mathrm{Wq}\right)+\operatorname{sum}(\mathrm{g} 1322 \mathrm{a}(\mathrm{i}) . * \mathrm{Wq})+\operatorname{sum}(\mathrm{g} 13\right.\right.$ $22 \mathrm{~b}(\mathrm{i}) . * \mathrm{Wq})))$

$\left(\mathrm{L}(3,9) *\left(\operatorname{sum}\left(\mathrm{g} 2313 \mathrm{a}(\mathrm{i}) .{ }^{*} \mathrm{Wq}\right)+\operatorname{sum}(\mathrm{g} 2313 \mathrm{~b}(\mathrm{i}) . * \mathrm{Wq})+\operatorname{sum}\left(\mathrm{g} 1323 \mathrm{a}(\mathrm{i}) .{ }^{*} \mathrm{Wq}\right)+\operatorname{sum}(\mathrm{g} 13\right.\right.$ $23 \mathrm{~b}(\mathrm{i}) \cdot * \mathrm{Wq})))) /(8 * \mathrm{pi})$;

\%Components of $\mathrm{S}_{\mathrm{ijkl}}$ are stored in the $\mathrm{S}$ matrix as below\%

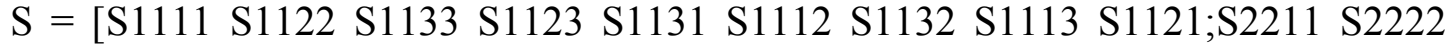
S2233 S2223 S2231 S2212 S2232 S2213 S2221;S3311 S3322 S3333 S3323 S3331 S3312 S3332 S3313 S3321;S2311 S2322 S2333 S2323 S2331 S2312 S2332 S2313 S2321;S3111 S3122 S3133 S3123 S3131 S3112 S3132 S3113 S3121;S1211 S1222 S1233 S1223 S1231 S1212 S1232 S1213 S1221;S3211 S3222 S3233 S3223 S3231 S3212 S3232 S3213 S3221;S1311 S1322 S1333 S1323 S1331 S1312 S1332 S1313 S1321;S2111 S2122 S2133 S2123 S2131 S2112 S2132 S2113 S2121]

\%This part encompasses the Mori Tanaka method developed earlier to compute the elastic properties of the final composite $\%$

for $\mathrm{Vf}=[0: 0.05: 0.25]^{*} 100 / 34$

$\mathrm{Vf}$

$\mathrm{x}=90 * \mathrm{pi} / 180$;

$\mathrm{m}=\cos (\mathrm{x})$;

$\mathrm{n}=\sin (\mathrm{x})$

$\mathrm{Vm}=1-\mathrm{Vf}$

$\mathrm{A}=\mathrm{I}+\mathrm{S} *(\mathrm{I} / \mathrm{L}) *(\mathrm{Cf} 1-\mathrm{L})$;

$\mathrm{Af}=\operatorname{inv}(\mathrm{A})$;

$\mathrm{C}=(\mathrm{L}+(\mathrm{Vf} *(\mathrm{Cf} 1-\mathrm{L}) * \mathrm{Af}) /(\mathrm{Vm} * \mathrm{I}+\mathrm{Vf} * \mathrm{Af})) ; \%$ mori tanaka formula\%

$\mathrm{Ss}=\operatorname{inv}(\mathrm{C}(1: 6,1: 6)) ; \%$ It is encouraged to use a matrix of order 6 to eliminate singularity and avoid errors $\%$

$\mathrm{E} 1=1 / \mathrm{Ss}(1,1)$ 


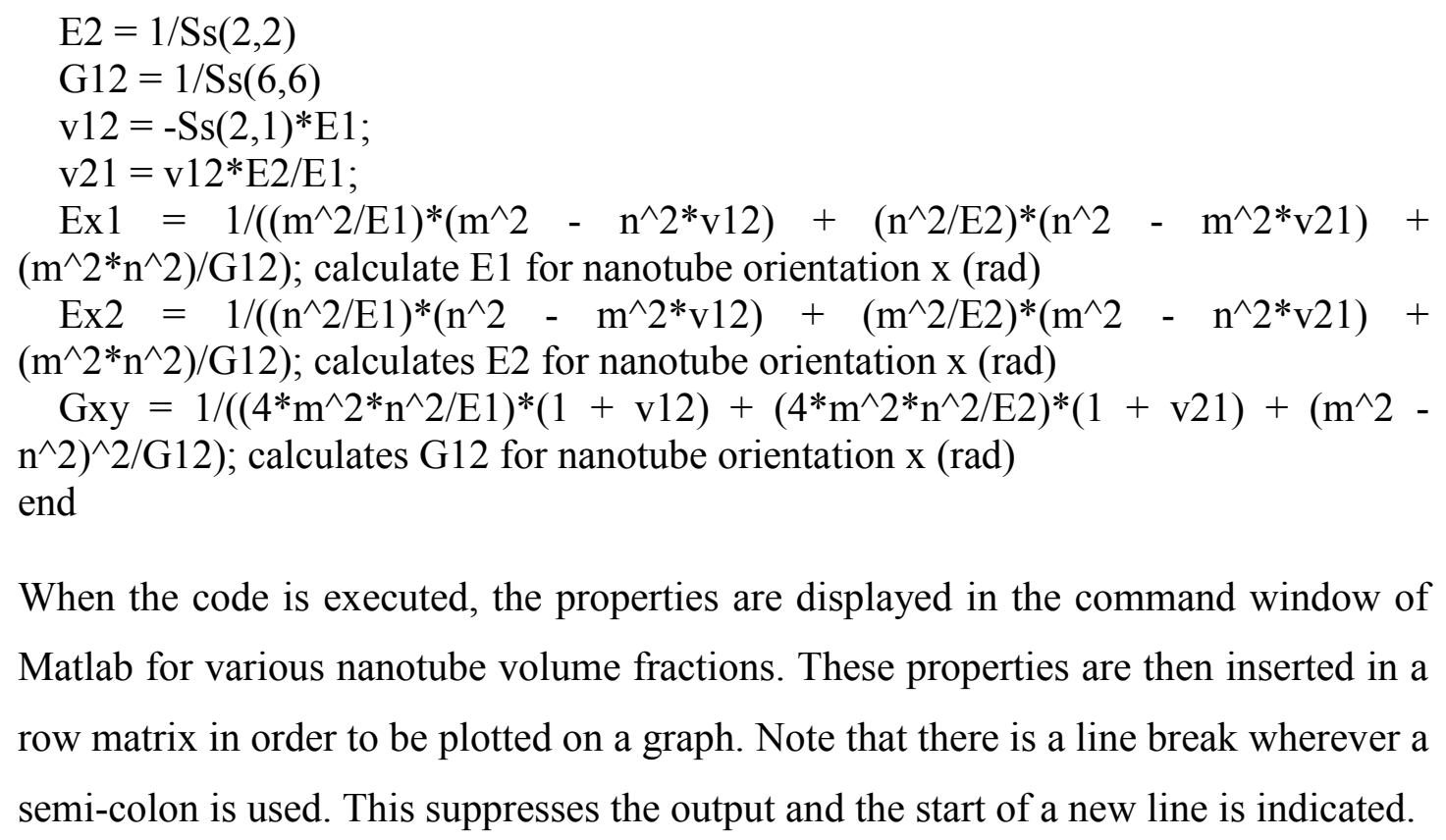

When the code is executed, the properties are displayed in the command window of Matlab for various nanotube volume fractions. These properties are then inserted in a row matrix in order to be plotted on a graph. Note that there is a line break wherever a semi-colon is used. This suppresses the output and the start of a new line is indicated. 


\subsection{Appendix C - License for figure 7.1}

This is a License Agreement between Sharil Patrale ("You") and Elsevier ("Elsevier") provided by Copyright Clearance Center ("CCC"). The license consists of your order details, the terms and conditions provided by Elsevier, and the payment terms and conditions.

All payments must be made in full to CCC. For payment instructions, please see information listed at the bottom of this form.

Supplier

Registered Company Number

Customer name

Customer address

License number

License date

Licensed content publisher

Licensed content publication

Licensed content title

Licensed content author

Licensed content date

Licensed content volume number

Licensed content issue number

Number of pages

Start Page

End Page
Elsevier Limited

The Boulevard,Langford Lane

Kidlington,Oxford,OX5 1GB,UK

1982084

Sharil Patrale

2112 C, Woodmar Drive

Houghton, MI 49931

3132680010496

Apr 19, 2013

Elsevier

Composites Science and Technology

Constitutive modeling of nanotubereinforced polymer composites

G.M. Odegard,T.S. Gates,K.E. Wise,C. Park,E.J. Siochi

August 2003

63

11

17

1671

1687 
Type of Use

Portion

Number of figures/tables/illustrations

Format

Are you the author of this Elsevier article?

Will you be translating?

Order reference number

Title of your thesis/dissertation

Expected completion date

Estimated size (number of pages)

Elsevier VAT number

Permissions price

VAT/Local Sales Tax

Total reuse in a thesis/dissertation

figures/tables/illustrations

\section{2}

both print and electronic

No

No

Multiscale modeling of liquid crystalline/nanotube composites

May 2013

105

GB 494627212

0.00 USD

$0.0 \mathrm{USD} / 0.0 \mathrm{GBP}$

0.00 USD

Terms and Conditions

\section{INTRODUCTION}

1. The publisher for this copyrighted material is Elsevier. By clicking "accept" in connection with completing this licensing transaction, you agree that the following terms and conditions apply to this transaction (along with the Billing and Payment terms and conditions established by Copyright Clearance Center, Inc. ("CCC"), at the time that you opened your Rightslink account and that are available at any time at http://myaccount.copyright.com).

\section{GENERAL TERMS}

2. Elsevier hereby grants you permission to reproduce the aforementioned material subject to the terms and conditions indicated.

3. Acknowledgement: If any part of the material to be used (for example, figures) 
has appeared in our publication with credit or acknowledgement to another source, permission must also be sought from that source. If such permission is not obtained then that material may not be included in your publication/copies. Suitable acknowledgement to the source must be made, either as a footnote or in a reference list at the end of your publication, as follows:

"Reprinted from Publication title, Vol /edition number, Author(s), Title of article / title of chapter, Pages No., Copyright (Year), with permission from Elsevier [OR APPLICABLE SOCIETY COPYRIGHT OWNER].” Also Lancet special credit "Reprinted from The Lancet, Vol. number, Author(s), Title of article, Pages No., Copyright (Year), with permission from Elsevier."

4. Reproduction of this material is confined to the purpose and/or media for which permission is hereby given.

5. Altering/Modifying Material: Not Permitted. However figures and illustrations may be altered/adapted minimally to serve your work. Any other abbreviations, additions, deletions and/or any other alterations shall be made only with prior written authorization of Elsevier Ltd. (Please contact Elsevier at permissions@elsevier.com)

6. If the permission fee for the requested use of our material is waived in this instance, please be advised that your future requests for Elsevier materials may attract a fee.

7. Reservation of Rights: Publisher reserves all rights not specifically granted in the combination of (i) the license details provided by you and accepted in the course of this licensing transaction, (ii) these terms and conditions and (iii) CCC's Billing and Payment terms and conditions.

8. License Contingent Upon Payment: While you may exercise the rights licensed immediately upon issuance of the license at the end of the licensing process for the transaction, provided that you have disclosed complete and accurate details of your proposed use, no license is finally effective unless and until full payment is received from you (either by publisher or by $\mathrm{CCC}$ ) as provided in CCC's Billing and Payment terms and conditions. If full payment is not received on a timely basis, then any license preliminarily granted shall be deemed automatically revoked and shall be void as if never granted. Further, in the event that you breach 
any of these terms and conditions or any of CCC's Billing and Payment terms and conditions, the license is automatically revoked and shall be void as if never granted. Use of materials as described in a revoked license, as well as any use of the materials beyond the scope of an unrevoked license, may constitute copyright infringement and publisher reserves the right to take any and all action to protect its copyright in the materials.

9. Warranties: Publisher makes no representations or warranties with respect to the licensed material.

10. Indemnity: You hereby indemnify and agree to hold harmless publisher and $\mathrm{CCC}$, and their respective officers, directors, employees and agents, from and against any and all claims arising out of your use of the licensed material other than as specifically authorized pursuant to this license.

11. No Transfer of License: This license is personal to you and may not be sublicensed, assigned, or transferred by you to any other person without publisher's written permission.

12. No Amendment Except in Writing: This license may not be amended except in a writing signed by both parties (or, in the case of publisher, by CCC on publisher's behalf).

13. Objection to Contrary Terms: Publisher hereby objects to any terms contained in any purchase order, acknowledgment, check endorsement or other writing prepared by you, which terms are inconsistent with these terms and conditions or CCC's Billing and Payment terms and conditions. These terms and conditions, together with CCC's Billing and Payment terms and conditions (which are incorporated herein), comprise the entire agreement between you and publisher (and CCC) concerning this licensing transaction. In the event of any conflict between your obligations established by these terms and conditions and those established by CCC's Billing and Payment terms and conditions, these terms and conditions shall control.

14. Revocation: Elsevier or Copyright Clearance Center may deny the permissions described in this License at their sole discretion, for any reason or no reason, with a full refund payable to you. Notice of such denial will be made using the contact information provided by you. Failure to receive such notice will not alter or 
invalidate the denial. In no event will Elsevier or Copyright Clearance Center be responsible or liable for any costs, expenses or damage incurred by you as a result of a denial of your permission request, other than a refund of the amount(s) paid by you to Elsevier and/or Copyright Clearance Center for denied permissions.

\section{LIMITED LICENSE}

The following terms and conditions apply only to specific license types:

15. Translation: This permission is granted for non-exclusive world English rights only unless your license was granted for translation rights. If you licensed translation rights you may only translate this content into the languages you requested. A professional translator must perform all translations and reproduce the content word for word preserving the integrity of the article. If this license is to re-use 1 or 2 figures then permission is granted for non-exclusive world rights in all languages.

16. Website: The following terms and conditions apply to electronic reserve and author websites:

Electronic reserve: If licensed material is to be posted to website, the web site is to be password-protected and made available only to bona fide students registered on a relevant course if:

This license was made in connection with a course, This permission is granted for 1 year only. You may obtain a license for future website posting, All content posted to the web site must maintain the copyright information line on the bottom of each image, A hyper-text must be included to the Homepage of the journal from which you are licensing at http://www.sciencedirect.com/science/journal/xxxxx or the Elsevier homepage for books at $h t t p: / / w w w . e l s e v i e r . c o m$, and Central Storage: This license does not include permission for a scanned version of the material to be stored in a central repository such as that provided by Heron/XanEdu.

17. Author website for journals with the following additional clauses:

All content posted to the web site must maintain the copyright information line on the bottom of each image, and the permission granted is limited to the personal 
version of your paper. You are not allowed to download and post the published electronic version of your article (whether PDF or HTML, proof or final version), nor may you scan the printed edition to create an electronic version. A hyper-text must be included to the Homepage of the journal from which you are licensing at http://www.sciencedirect.com/science/journal/xxxxx . As part of our normal production process, you will receive an e-mail notice when your article appears on Elsevier's online service ScienceDirect (www.sciencedirect.com). That e-mail will include the article's Digital Object Identifier (DOI). This number provides the electronic link to the published article and should be included in the posting of your personal version. We ask that you wait until you receive this e-mail and have the DOI to do any posting.

Central Storage: This license does not include permission for a scanned version of the material to be stored in a central repository such as that provided by Heron/XanEdu.

18. Author website for books with the following additional clauses: Authors are permitted to place a brief summary of their work online only. A hyper-text must be included to the Elsevier homepage athttp://www.elsevier.com . All content posted to the web site must maintain the copyright information line on the bottom of each image. You are not allowed to download and post the published electronic version of your chapter, nor may you scan the printed edition to create an electronic version.

Central Storage: This license does not include permission for a scanned version of the material to be stored in a central repository such as that provided by Heron/XanEdu.

19. Website (regular and for author): A hyper-text must be included to the Homepage of the journal from which you are licensing athttp://www.sciencedirect.com/science/journal/xxxxx. or for books to the Elsevier homepage at http://www.elsevier.com

20. Thesis/Dissertation: If your license is for use in a thesis/dissertation your thesis may be submitted to your institution in either print or electronic form. Should your thesis be published commercially, please reapply for permission. These requirements include permission for the Library and Archives of Canada to supply single copies, on demand, of the complete thesis and include permission for 
UMI to supply single copies, on demand, of the complete thesis. Should your thesis be published commercially, please reapply for permission.

\section{Other Conditions:}

v1.6

If you would like to pay for this license now, please remit this license along with your payment made payable to "COPYRIGHT CLEARANCE CENTER" otherwise you will be invoiced within 48 hours of the license date. Payment should be in the form of a check or money order referencing your account number and this invoice number 501003912. Once you receive your invoice for this order, you may pay your invoice by credit card. Please follow the instructions provided at that time.

Make Payment To:

Copyright Clearance Center

Dept 001

P. O. Box 843006

Boston, MA 02284-3006

For suggestions or comments regarding this order, contact Rightslink Customer Support: customercare@copyright.com or $+1-877-622-5543$ (toll free in the US) or $+1-978-646-2777$.

Gratis licenses (referencing $\$ 0$ in the Total field) are free. Please retain this printable license for your reference. No payment is required. 